\title{
Mitochondria, lipotoxicity and skeletal muscle metabolism : implications for type 2 diabetes mellitus
}

Citation for published version (APA):

van Bree, B. W. J. (2014). Mitochondria, lipotoxicity and skeletal muscle metabolism : implications for type 2 diabetes mellitus. [Doctoral Thesis, Maastricht University]. Uitgeverij BOXPress.

https://doi.org/10.26481/dis.20141017bb

Document status and date:

Published: 01/01/2014

DOI:

10.26481/dis.20141017bb

Document Version:

Publisher's PDF, also known as Version of record

\section{Please check the document version of this publication:}

- A submitted manuscript is the version of the article upon submission and before peer-review. There can be important differences between the submitted version and the official published version of record.

People interested in the research are advised to contact the author for the final version of the publication, or visit the DOI to the publisher's website.

- The final author version and the galley proof are versions of the publication after peer review.

- The final published version features the final layout of the paper including the volume, issue and page numbers.

Link to publication

\footnotetext{
General rights rights.

- You may freely distribute the URL identifying the publication in the public portal. please follow below link for the End User Agreement:

www.umlib.nl/taverne-license

Take down policy

If you believe that this document breaches copyright please contact us at:

repository@maastrichtuniversity.nl

providing details and we will investigate your claim.
}

Copyright and moral rights for the publications made accessible in the public portal are retained by the authors and/or other copyright owners and it is a condition of accessing publications that users recognise and abide by the legal requirements associated with these

- Users may download and print one copy of any publication from the public portal for the purpose of private study or research.

- You may not further distribute the material or use it for any profit-making activity or commercial gain

If the publication is distributed under the terms of Article $25 \mathrm{fa}$ of the Dutch Copyright Act, indicated by the "Taverne" license above, 
Mitochondria, lipotoxicity and skeletal muscle metabolism: implications for type 2 diabetes mellitus 


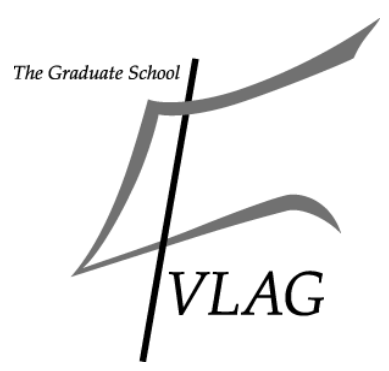

The study presented in this thesis was performed within NUTRIM school for Nutrition, Toxicology and Metabolism which participates in the Graduate School VLAG (Food Technology, Agrobiotechnology, Nutrition and Health Science), accredited by the Royal Netherlands Academy of Arts and Sciences.

Cover design: Felice Duker, Bianca van Bree Lay-out: Bianca van Bree

Printed by: Proefschriftmaken.nl/Uitgeverij BOXPress

ISBN: 978-90-8891-953-4

(c) Copyright Bianca van Bree, Maastricht 2014 


\section{Mitochondria, lipotoxicity and skeletal muscle metabolism: implications for type 2 diabetes mellitus}

\section{Proefschrift}

ter verkrijging van de graad van doctor aan de Universiteit Maastricht, op gezag van de Rector Magnificus,

Prof. Dr. L.L.G. Soete,

volgens het besluit van het College van Decanen,

in het openbaar te verdedigen

op vrijdag 17 oktober 2014 om 10.00 uur

door

\section{Bianca Wilhelmina Johanna van Bree}

geboren te Boxmeer op 14 augustus 1984 


\section{PROMOTOREN}

Prof. dr. L.P.A.J. Schrauwen

Prof. dr. M.K.C. Hesselink

\section{COPROMOTER}

Dr. J. Hoeks

\section{BEOORDELINGSCOMMISSIE}

Prof. dr. W. van Marken Lichtenbelt (voorzitter)

Prof. dr. A. Bast

Dr. R.H.L. Houtkooper (Academisch Medisch Centrum Amsterdam)

Prof. dr. J. Keijer (Wageningen University)

Dr. J.J.F.P. Luiken 


\section{Contents}

Chapter 1

General Introduction

Chapter 2

Targeting of mitochondrial reactive oxygen species production does not avert lipidinduced insulin resistance in muscle tissue from mice

\section{Chapter 3}

A two-week treatment with 2,4-dinitrophenol does not affect muscle substrate oxidation and glucose homeostasis in WT and $\mathrm{db} / \mathrm{db}$ mice

\section{Chapter 4}

A genistein-enriched diet does not improve skeletal muscle oxidative capacity or prevent the transition toward advanced stages of insulin resistance in ZDF rats

\section{Chapter 5}

The mitochondrial protein mitoNEET: a study of its role in mitochondrial oxidative capacity, substrate metabolism, and lipid accumulation in skeletal muscle in rats

Chapter 6

Lack of UCP3 does not affect skeletal muscle mitochondrial function under lipidchallenged conditions, but leads to sudden cardiac death

\section{Chapter 7}

General Discussion

Summary - Samenvatting

Valorization

Dankwoord 

CHAPTER 1

General Introduction 
Every cell in living organisms depends on adenosine triphosphate (ATP) as a primary energy source required for biochemical and physiological processes. Mitochondria are the most important organelles for energy production by generating ATP through the electron transport chain (ETC) located in the inner mitochondrial membrane (IMM). Via the food we eat, especially carbohydrates and lipids, substrates for mitochondria are provided that can be used for ATP production. These substrates are mainly degraded in the mitochondrial matrix, the center of mitochondria that is surrounded by the IMM. During degradation of nutrients in the mitochondrial matrix, the electron donors $\mathrm{NADH}$ and $\mathrm{FADH}_{2}$ are formed. Subsequently, $\mathrm{NADH}$ and $\mathrm{FADH}_{2}$ donate their high-energy electrons to the ETC which passes on the electrons to the final electron acceptor oxygen [1]. The ETC system consists of five protein complexes: complex I to $\mathrm{V}$. The energy released from the electron transfer is used by complexes I, III, and IV to translocate protons from the mitochondrial matrix to the mitochondrial intermembrane space. As a result, a proton gradient across the IMM is generated. This proton gradient can be used directly to drive the activity of complex $\mathrm{V}$, an ATP synthase that generates ATP via the phosphorylation of ADP [2]. Thus, oxidation of substrates, such as fatty acids and glucose, is thereby coupled to ATP production, a process to which is referred as oxidative phosphorylation. However, the coupling between substrate oxidation, the mitochondrial proton gradient, and ATP production is not $100 \%$ efficient. A process termed 'mitochondrial uncoupling' refers to the re-entry of protons into the matrix dissociated from ATP synthase, for instance due to the presence of uncoupling proteins [3] or adenine nucleotide transferase (ANT) [4]. Consequently, mitochondrial uncoupling could lead to less efficient ATP production and might thus affect energy expenditure [5].

\section{Compromised mitochondrial function in type 2 diabetes}

Due to their relevance in cellular metabolism, defects in mitochondrial function are linked with various diseases, such as type 2 diabetes (T2DM), cancer, and chronic liver diseases. T2DM is one of the most common chronic diseases of this time, and is an important cause of morbidity and mortality worldwide [6, 7]. One of the hallmarks in the early onset of T2DM is insulin resistance. Since skeletal muscle is a key insulin-responsive organ responsible for maintaining normal glucose homeostasis, its transition towards an insulin resistant state is an important contributor to the disturbances in glucose metabolism found in T2DM patients. Mitochondrial dysfunction is suggested to contribute to the development of insulin resistance of skeletal muscle. In this context, reduced mitochondrial size and a diminished oxidative capacity have been revealed in skeletal muscle of T2DM patients and non-diabetic individuals with a family history of T2DM [8-10]. Although mitochondrial dysfunction has been reported repeatedly in insulin-resistant subjects and type 2 diabetics, the exact (causal) role 
in whole-body glucose homeostasis and progression of insulin resistance is less clear. Also, it is unclear which factors are involved in impairing mitochondrial function.

\section{Mitochondrial oxidative capacity and lipotoxicity}

Predisposing risk factors for T2DM comprise obesity, increased dietary fat intake, and a sedentary lifestyle. Consumption of calories that exceed daily energy expenditure results in a positive energy balance. Because adipose tissue, the main fat depot in the human body, does not have an infinite capacity to store fat, lipids can spill over to non-adipose tissues such as skeletal muscle [11]. Excess lipid accumulation in skeletal muscle can result in toxic effects on its function and is therefore also referred to as lipotoxicity [12, 13]. Indeed, lipotoxicity is strongly associated with insulin resistance [14]. Because mitochondria are the primary cellular site for fatty acid oxidation and utilization, strategies to improve mitochondrial oxidative capacity might prevent lipotoxicity and thereby enhance insulin sensitivity $[15,16]$.

\section{Mitochondrial production of reactive oxygen species and lipotoxicity}

Importantly, the process of oxidative phosphorylation not only produces ATP, but also generates potentially harmful reactive oxygen species (ROS). ROS are continuously produced and scavenged in order to maintain ROS at certain steady-state levels. Moderate concentrations of ROS are required for normal physiological processes such as cell signaling and immune responses. However, when the balance between ROS generation and scavenging is disturbed, excessive levels of ROS could react with cellular components such as lipids, proteins, and nucleic acids. Hereby, ROS can generate oxidative modifications, also recognized as oxidative damage, which could lead to disturbed mitochondrial function, insulin signaling, and eventually progression of insulin resistance and T2DM [17-19]. Indeed, oxidative damage markers in skeletal muscle and insulin resistance are tightly correlated [18, 20-23]. In skeletal muscle, especially lipids accumulated in the vicinity of mitochondria are highly susceptible to ROS-induced oxidative damage [24]. ROS can directly lead to lipid peroxidation and formation of highly reactive aldehyde species such as 4-hydroxy-2,3,transnonenal (4HNE). Especially fatty acids that are accumulated in the IMM, the main site where ROS are generated, are prone to peroxidation, which in turn could damage the components of the oxidative phosphorylation system [25], contributing further to reduced mitochondrial capacity. Indeed, previous rodent studies have shown that high-fat diet induced insulin resistance was linked with skeletal muscle mitochondrial dysfunction as a consequence of hyperglycemia- and hyperlipidemia-induced ROS production [21]. In addition, lipids accumulated in skeletal muscle of obese insulin resistant subjects have been reported to show a higher degree of lipid peroxidation [26]. Thus, excessive ROS production provides a 


\section{CHAPTER 1}

likely link between muscular fat accumulation, mitochondrial dysfunction and insulin resistance.

\section{Outline of this thesis}

This thesis mainly focuses on the link between mitochondrial oxidative capacity, ROS, and glucose homeostasis. A complex web of factors such as nutrient overload, mitochondrial dysfunction, and insulin resistance, could result in metabolic abnormalities, ultimately leading to diseases such as T2DM. Defects in mitochondrial function often seen in T2DM patients are not an irreversible lesion per se. In this perspective, mitochondrial oxidative capacity has been shown to improve upon calorie restriction and aerobic exercise in obese and insulin resistance subjects [27]. In this thesis, we have used different approaches to manipulate aspects of mitochondrial function and muscle substrate metabolism, and studied the effect on glucose tolerance and muscle lipid content.

In chapter 2 we investigated the possible involvement of mitochondrial ROS production in the development of insulin resistance. By specifically targeting mitochondrial ROS with a derivative of plastoquinone (SkQ) in a mouse model of diet-induced obesity we studied whether blunting ROS levels could improve glucose homeostasis.

Mitochondrial uncoupling, a process in which the mitochondrial proton gradient is dissociated from ATP production, has been hypothesized to limit mitochondrial ROS production, prevent mitochondrial dysfunction and to increase metabolic rate and insulin action. In chapter $\mathbf{3}$ we investigated if chemical uncoupling would lead to beneficial health effects with respect to glucose metabolism. Therefore, a group of healthy mice and a group of insulin resistant mice were subjected to a 2-week intervention of oral administration of a chemical uncoupling compound, i.e. 2,4-dinitrophenol (DNP). The effects of DNP on muscle substrate accumulation, lipid accumulation, and whole-body glucose tolerance were measured.

Genistein, a natural food compound mainly present in soybeans, has also been reported to improve skeletal muscle oxidative metabolism and thereby stimulating glucose uptake and fatty acid handling. In addition, genistein was shown to have antioxidant activities. Therefore, in chapter 4 we evaluated whether genistein could antagonize the progression of the hyperinsulinemic normoglycemic state (pre-diabetes) toward full-blown T2DM. Rats that are prone to develop insulin resistance were supplemented with genistein and muscle oxidative capacity, ROS, and whole-body glucose tolerance were studied.

In chapter 5 we used a genetic approach to manipulate mitochondrial oxidative capacity. MitoNEET has been identified as a potent regulator of mitochondrial oxidative capacity, and consequently, may impact substrate metabolism. Therefore, we overexpressed mitoNEET specifically in skeletal muscle of rats via in vivo gene electroporation and 
examined the effects on mitochondrial oxidative capacity and intramyocellular lipid accumulation.

Chapter 6 focusses on the role of the mitochondrial uncoupling protein 3 (UCP3) in mitochondrial lipotoxicity. UCP3 was suggested to prevent mitochondrial lipotoxicity and lower mitochondrial ROS. In chapter 6, we examined the effect of UCP3 ablation in mice that received a high-fat diet combined with a fat oxidation inhibitor (etomoxir), in order to maximally disturb the balance between fat supply and fat oxidative capacity.

Finally, in chapter 7 the main findings obtained in this thesis are discussed and placed in a broader perspective. Implications for further research are presented.

\section{REFERENCES}

1. Papa, S., et al., The oxidative phosphorylation system in mammalian mitochondria. Adv Exp Med Biol, 2012. 942: p. 3-37.

2. Mitchell, P., Coupling of phosphorylation to electron and hydrogen transfer by a chemiosmotic type of mechanism. Nature, 1961. 191: p. 144-8.

3. Schrauwen, P., J. Hoeks, and M.K. Hesselink, Putative function and physiological relevance of the mitochondrial uncoupling protein-3: involvement in fatty acid metabolism? Prog Lipid Res, 2006. 45(1): p. 17-41.

4. Brand, M.D., et al., The basal proton conductance of mitochondria depends on adenine nucleotide translocase content. Biochem J, 2005. 392(Pt 2): p. 353-62.

5. Thrush, A.B., et al., Implications of mitochondrial uncoupling in skeletal muscle in the development and treatment of obesity. FEBS J, 2013. 280(20): p. 5015-29.

6. Danaei, G., et al., National, regional, and global trends in fasting plasma glucose and diabetes prevalence since 1980: systematic analysis of health examination surveys and epidemiological studies with 370 country-years and 2.7 million participants. Lancet, 2011. 378(9785): p. 31-40.

7. Roglic, G. and N. Unwin, Mortality attributable to diabetes: estimates for the year 2010. Diabetes Res Clin Pract, 2010. 87(1): p. 15-9.

8. Kelley, D.E., et al., Dysfunction of mitochondria in human skeletal muscle in type 2 diabetes. Diabetes, 2002. 51(10): p. 2944-50.

9. Patti, M.E., et al., Coordinated reduction of genes of oxidative metabolism in humans with insulin resistance and diabetes: Potential role of PGC1 and NRF1. Proc Natl Acad Sci U S A, 2003. 100(14): p. 8466-71.

10. Petersen, K.F., et al., Impaired mitochondrial activity in the insulin-resistant offspring of patients with type 2 diabetes. N Engl J Med, 2004. 350(7): p. 664-71.

11. Boden, G., et al., Effects of acute changes of plasma free fatty acids on intramyocellular fat content and insulin resistance in healthy subjects. Diabetes, 2001. 50(7): p. 1612-7.

12. Hoeks, J., et al., Peroxisome proliferator-activated receptor-gamma coactivator-1 and insulin resistance: acute effect of fatty acids. Diabetologia, 2006. 49(10): p. 2419-26.

13. Schaffer, J.E., Lipotoxicity: when tissues overeat. Curr Opin Lipidol, 2003. 14(3): p. 281-7.

14. Consitt, L.A., J.A. Bell, and J.A. Houmard, Intramuscular lipid metabolism, insulin action, and obesity. IUBMB Life, 2009. 61(1): p. 47-55.

15. Meex, R.C., et al., Restoration of muscle mitochondrial function and metabolic flexibility in type 2 diabetes by exercise training is paralleled by increased myocellular fat storage and improved insulin sensitivity. Diabetes, 2010. 59(3): p. 572-9.

16. Timmers, S., et al., Calorie restriction-like effects of 30 days of resveratrol supplementation on energy metabolism and metabolic profile in obese humans. Cell Metab, 2011. 14(5): p. 612-22. 
17. Rains, J.L. and S.K. Jain, Oxidative stress, insulin signaling, and diabetes. Free Radic Biol Med, 2011. 50(5): p. 567-75.

18. Kumashiro, N., et al., Impact of oxidative stress and peroxisome proliferator-activated receptor gamma coactivator-1alpha in hepatic insulin resistance. Diabetes, 2008. 57(8): p. 2083-91.

19. Kowaltowski, A.J. and A.E. Vercesi, Mitochondrial damage induced by conditions of oxidative stress. Free Radic Biol Med, 1999. 26(3-4): p. 463-71.

20. Anderson, E.J., et al., Mitochondrial $\mathrm{H} 2 \mathrm{O} 2$ emission and cellular redox state link excess fat intake to insulin resistance in both rodents and humans. J Clin Invest, 2009. 119(3): p. 573-81.

21. Bonnard, C., et al., Mitochondrial dysfunction results from oxidative stress in the skeletal muscle of diet-induced insulin-resistant mice. J Clin Invest, 2008. 118(2): p. 789-800.

22. Houstis, N., E.D. Rosen, and E.S. Lander, Reactive oxygen species have a causal role in multiple forms of insulin resistance. Nature, 2006. 440(7086): p. 944-8.

23. Lefort, N., et al., Increased reactive oxygen species production and lower abundance of complex I subunits and carnitine palmitoyltransferase 1B protein despite normal mitochondrial respiration in insulin-resistant human skeletal muscle. Diabetes, 2010. 59(10): p. 2444-52.

24. Schrauwen, P., et al., Mitochondrial dysfunction and lipotoxicity. Biochim Biophys Acta, 2010. 1801(3): p. 266-71.

25. Lippe, G., et al., The inactivation of mitochondrial F1 ATPase by $\mathrm{H} 2 \mathrm{O} 2$ is mediated by iron ions not tightly bound in the protein. Biochem Biophys Res Commun, 1991. 181(2): p. 764-70.

26. Russell, A.P., et al., Lipid peroxidation in skeletal muscle of obese as compared to endurance-trained humans: a case of good vs. bad lipids? FEBS Lett, 2003. 551(1-3): p. 104-6.

27. Menshikova, E.V., et al., Effects of weight loss and physical activity on skeletal muscle mitochondrial function in obesity. Am J Physiol Endocrinol Metab, 2005. 288(4): p. E818-25. 


\section{CHAPTER 2}

\section{Targeting of mitochondrial reactive oxygen species production does not avert lipid- induced insulin resistance in muscle tissue from mice}

B. van Bree*, S. Paglialunga*, M. Bosma, M.P. Valdecantos, E. Amengual-Cladera, J.A. Jörgensen, D. van Beurden, G.J.M. den Hartog, D.M. Ouwens, J.J. Briedé, P. Schrauwen, J. Hoeks 


\section{ABSTRACT}

Aims/hypothesis High-fat, high-sucrose diet (HF)-induced reactive oxygen species (ROS) levels are implicated in skeletal muscle insulin resistance and mitochondrial dysfunction. Here we investigated whether mitochondrial ROS sequestering can circumvent HF-induced oxidative stress; we also determined the impact of any reduced oxidative stress on muscle insulin sensitivity and mitochondrial function.

Methods Skulachev ion (plastoquinonyl decyltriphenylphosphonium) (SkQ), a mitochondriaspecific antioxidant, was used to target ROS production in $\mathrm{C} 2 \mathrm{C} 12$ muscle cells as well as in HF-fed (16 weeks old) male C57BI/6 mice, compared with mice on low-fat chow diet (LF) or $\mathrm{HF}$ alone. Oxidative stress was measured as protein carbonylation levels. Glucose tolerance tests, glucose uptake assays and insulin-stimulated signalling were determined to assess muscle insulin sensitivity. Mitochondrial function was determined by high-resolution respirometry.

Results SkQ treatment reduced oxidative stress in muscle cells $(-23 \% p<0.05)$, but did not improve insulin sensitivity and glucose uptake under insulin-resistant conditions. In HF mice, oxidative stress was elevated ( $56 \%$ vs LF $\mathrm{p}<0.05)$, an effect completely blunted by $S k Q$. However, HF and HF+SkQ mice displayed impaired glucose tolerance (AUC HF up 33\%, $\mathrm{p}<0.001 ; \mathrm{HF}+\mathrm{SkQ}$ up $22 \% ; \mathrm{p}<0.01$ vs LF) and disrupted skeletal muscle insulin signalling. ROS sequestering did not improve mitochondrial function.

Conclusions/interpretation SkQ treatment reduced muscle mitochondrial ROS production and prevented HF-induced oxidative stress. Nonetheless, whole-body glucose tolerance, insulinstimulated glucose uptake, muscle insulin signalling and mitochondrial function were not improved. These results suggest that HF-induced oxidative stress is not a prerequisite for the development of muscle insulin resistance. 


\section{INTRODUCTION}

Type 2 diabetes has been associated with oxidative stress, a condition that arises when production of reactive oxygen species (ROS) exceeds the antioxidant defence system's capacity. Several studies have shown that skeletal muscle mitochondrial ROS production is increased in insulin-resistant conditions [1, 2], which are an early hallmark in the development of type 2 diabetes. Moreover, it has been suggested that ROS and oxidative stress are causal factors of skeletal muscle insulin resistance [3, 4].

Lee et al [5] recently showed that age-induced development of insulin resistance, which is associated with increases in ROS production and oxidative stress in mice, could be prevented when mitochondrial $\mathrm{H}_{2} \mathrm{O}_{2}$ release was attenuated by overexpression of the gene encoding mitochondrial catalase (MCAT), which also prevented the development of mitochondrial dysfunction observed upon ageing. Interestingly, type 2 diabetic patients also have mitochondrial dysfunction, as exemplified by smaller and damaged mitochondria [6], reduced in vivo muscle ATP production capacity $[7,8]$ and lowered mitochondrial density [9, 10]. Furthermore, we have previously demonstrated that ex vivo intrinsic skeletal muscle mitochondrial respiration [11] and in vivo mitochondrial oxidative capacity [8] are reduced in patients with type 2 diabetes. Taken together, these findings suggest that elevated ROS and oxidative stress in skeletal muscle are implicated in insulin resistance and may be the underlying cause of the mitochondrial dysfunction observed in type 2 diabetes.

Therefore, we investigated whether the targeting of mitochondrial ROS production in an in vitro and in vivo model of lipid-induced insulin resistance could alleviate insulin resistance by lowering muscle oxidative stress. For this purpose, we used a novel oral mitochondria-specific antioxidant, the Skulachev ion (plastoquinonyl decyltriphenylphosphonium) (SkQ). SkQ is a small cationic molecule that targets and accumulates in the inner mitochondrial membrane [12]. SkQ mainly scavenges superoxide radicals arising from complex I, but can also sequester ROS generated from complex III [13], thus preventing excessive production of mitochondria-generated ROS. In vitro studies have shown that SkQ has a wide concentration range of antioxidant capacity [13], and in vivo studies have demonstrated that $S k Q$ treatment effectively reduced oxidative damage in several models of oxidative stress [12-14].

In the present study we used the SkQ compound to: (1) determine whether sequestering of mitochondrial ROS production can prevent increased ROS production and oxidative stress in skeletal muscle under high-fat conditions; and (2) examine the impact of the anticipated lowering of oxidative stress on skeletal muscle insulin sensitivity and mitochondrial function. 


\section{MATERIALS AND METHODS}

\section{Chemicals}

SkQ was generously donated by O. Fedorkin (Mitotech, Moscow, Russia). All other chemicals were purchased from Sigma (St Louis, MO, USA) unless otherwise stated.

\section{C2C12 cell glucose uptake assay}

C2C12 cells (LGC standards, Teddington, UK) were maintained in DMEM (GlutaMAX lowglucose DMEM; Invitrogen, Breda, the Netherlands) supplemented with 10\% FCS (vol./vol.) and grown on ECM (extracellular matrix) matrigel-coated culture plates. Cells were differentiated towards multinucleated myotubes over the course of a week in $\alpha \mathrm{MEM}$ supplemented with 2\% FCS (vol./vol.). Differentiated myotubes were pretreated with 20 $\mathrm{nmol} / / \mathrm{SkQ}$ or vehicle (control) during the last 3 days of differentiation before collection. For the oxidative stress assay, myotubes were lysed in RIPA buffer and protein carbonyls were determined as described below. Prior to the glucose uptake assays, the cells were treated for $24 \mathrm{~h}$ with $500 \mu \mathrm{mol} / \mathrm{l}$ palmitate conjugated to BSA (essential NEFA-free) (ratio BSA:palmitate $1: 2.5)$ or solely BSA (control). Deoxyglucose uptake was performed as previously described [15].

\section{In vivo mouse study}

Male C57BI/6 mice ( 8 weeks old; $n=10$ per group) were purchased from Charles River (Maastricht, the Netherlands) and housed individually on a $12 \mathrm{~h}$ light: $12 \mathrm{~h}$ dark cycle. The mice were placed on either a low-fat chow diet (LF) (Ssniff, Soest, Germany) or a high-fat, high-sucrose diet (HF) (Research Diets, New Brunswick, NJ, USA) for 16 weeks. A third group of mice received the $\mathrm{HF}$ plus the $\mathrm{SkQ}$ antioxidant $(\mathrm{HF}+\mathrm{SkQ})$. Based on previous studies, an SkQ dose of $250 \mathrm{nmol} / \mathrm{kg}$ body weight [16] was supplemented throughout the study via the drinking water [17]. Water bottles for all mice were refreshed three times per week, and body weight and food intake were measured weekly. After the 16 week dietary intervention period, mice were fasted for $3 \mathrm{~h}$ and killed by cervical dislocation under basal control $(n=5)$ conditions or after $10 \mathrm{~min}$ of insulin (Actrapid; Novo Nordisk, Bagsvaerd, Denmark) stimulation (10 U/kg i.p.; $n=5$ ). Skeletal muscles were excised, frozen in liquid nitrogen-cooled isopentane (2-methyl-butane; Fluka, Zwijndrecht, the Netherlands) and stored at $-80^{\circ} \mathrm{C}$ until further analysis. All protocols were approved and conducted in accordance with Maastricht University Animal Ethics Committee guidelines. 


\section{Glucose tolerance tests and measurements from plasma}

Glucose tolerance tests were performed after week 15 of the intervention, following a $5 \mathrm{~h}$ fast as previously described [18]. Blood glucose was measured using a glucose meter (LifeScan, Milpitas, CA, USA) and samples were collected in microtitre tubes (BD, Franklin Lakes, NJ, USA). Plasma was separated by centrifugation for $5 \mathrm{~min}$ at $5,000 \mathrm{~g}$ and stored at $-80^{\circ} \mathrm{C}$. Plasma insulin was measured using a commercially available RIA kit (Millipore, Billerica, MA, USA). Plasma triacylglycerol and NEFA levels were determined with commercially available kits from Roche (Schlieren, Switzerland) and Wako Chemicals (Neuss, Germany), respectively.

\section{Western blot}

To determine (phosphorylated)Akt and oxidative phosphorylation (OXPHOS) proteins, gastrocnemius muscle was homogenised in lysis buffer (10\% NP40 [vol./vol.], 10\% SDS [vol./vol.], $100 \mathrm{mmol} / / \mathrm{PMSF}$ in PBS) and cells were lysed in RIPA buffer supplemented with protease inhibitor cocktail (Roche). Equal amounts of protein were loaded on to SDS-PAGE gel, and standard running, blocking and incubation protocols were followed using primary antibodies against Akt, phosphorylated Akt (pAkt) (S473) (Cell Signaling Technology, Danvers, MA, USA), OXPHOS proteins (Mitosciences, Eugene, OR, USA), sarcomeric actin (Santa Cruz Biotechnology, Santa Cruz, CA, USA) or $\beta$-actin. The appropriate secondary antibodies (Invitrogen, Paisley, UK) were applied and blots were visualised using the Odyssey Near Infrared Imager (Licor, Leusden, the Netherlands) or with a maximum sensitivity substrate (SuperSignal West Femto; Thermo Fisher Scientific, Waltham, MA, USA) in a reader (ChemiDoc XRS; BioRad, Hercules, CA, USA). For glycogen synthase kinase 3 (GSK3) and JUN N-terminal kinase (JNK) phosphorylation, and IRS1 content levels, muscle samples were homogenised in Bioplex lysis buffer containing protease and phosphatase inhibitors (Biorad), after which protein content was determined using a BCA-kit (Thermo Scientific, Rockford, IL, USA). Protein (10 $\mu \mathrm{g})$ was loaded on to SDS-PAGE gels, and standard blocking and incubation protocols were followed using primary antibodies against phospho-GSK3 $\alpha / \beta-S e r 21 / 9$ and phospho-JNK-Thr183/Tyr185 (Cell Signaling Technology), or against IRS1 [19]. Loading corrections for phosphorylation levels were performed by reprobing with antibodies recognising $\alpha$-tubulin (Calbiochem, Darmstadt, Germany) and glyceraldehyde 3-phosphate dehydrogenase (GAPDH) (Cell Signaling Technology). Bound antibodies were detected with horseradish-peroxidase-conjugated secondary antibodies, followed by enhanced chemiluminescence, and visualised using the Versadoc system (Biorad). Blots were quantified using a software package (Quantity One, version 4.6.9; Biorad). Protein carbonyl levels were assessed with a protein oxidation detection kit (Oxyblot; Millipore) as described elsewhere [20]. 


\section{Mitochondrial DNA copy number}

The mitochondrial DNA copy number was determined by the ratio of Cox2 expression (mitochondrial gene) over Ucp2 expression (nuclear gene) as previously described [21]. Briefly, isolated DNA (Nucleospin Tissue kit; Macherey Nagel, Düren, Germany) from gastrocnemius muscle samples was analysed by real-time PCR using a sequence detector (ABI 7900; Applied Biosystems, Branchburg, NJ, USA) and the following program: one cycle at $50^{\circ} \mathrm{C}$ for $2 \mathrm{~min}$ then at $95^{\circ} \mathrm{C}$ for $10 \mathrm{~min}$, followed by 40 cycles at $95^{\circ} \mathrm{C}$ for $15 \mathrm{~s}$ and $60^{\circ} \mathrm{C}$ for $1 \mathrm{~min}$. The absolute quantification of each gene was determined by a standard curve.

\section{Enzyme activities}

The activity of superoxide dismutase 2 (SOD2) and glutathione peroxidase 1 (GPx1) was measured in quadriceps muscle homogenates. SOD2 activity was determined according to Oberley and Spitz [22] and distinguished from total SOD activity by pre-incubating the samples with $\mathrm{NaCN}$ ( $5 \mathrm{mmol} / \mathrm{l})$ for $30 \mathrm{~min}$. GPx1 activity was determined according to Paglia and Valentine [23] with reduced glutathione at a concentration of $10 \mathrm{mmol} / \mathrm{l}$. The activity of hydroxylacyl dehydrogenase $(\mathrm{HADH})$ and citrate synthase (CS) was measured in gastrocnemius muscle homogenates as previously described [24].

\section{Mitochondrial isolation, respiration and ROS measurements}

In a second set of mice ( $\mathrm{n}=7$ per group) on the same diet and intervention regimen as that described above, skeletal muscle mitochondria were isolated and respiration was effected as previously described $[25,26]$. Respiration rates in isolated mitochondria $(0.1 \mathrm{mg} / \mathrm{ml})$ were determined at $37^{\circ} \mathrm{C}$ by polarographic oxygen sensors in a two-chamber Oxygraph (Oroboros Instruments, Innsbruck, Austria) using pyruvate $(5 \mathrm{mmol} / \mathrm{l})$ plus malate $(3 \mathrm{mmol} / \mathrm{l})$, or palmitoyl-coenzyme A (CoA) $(50 \mu \mathrm{mol} / \mathrm{l})$ plus carnitine $(2 \mathrm{mmol} / \mathrm{l})$ as substrates. For mitochondrial ROS production, we evaluated $\mathrm{H}_{2} \mathrm{O}_{2}$ release from isolated mitochondria over $15 \mathrm{~min}$ at $37^{\circ} \mathrm{C}$ using Amplex Red fluorescence quantification (Invitrogen). Briefly, mitochondria $(50 \mu \mathrm{g})$ were added to respiration buffer $(100 \mathrm{mmol} / \mathrm{l}$ sucrose, $50 \mathrm{mmol} / \mathrm{KCl}$, $20 \mathrm{mmol} / \mathrm{l}$ TES (2-[[1,3-dihydroxy-2-(hydroxymethyl)propan-2-yl]amino]ethanesulfonic acid), $1 \mathrm{mmol} / / \mathrm{EDTA}, 4 \mathrm{mmol} / \mathrm{l} \mathrm{KH}_{2} \mathrm{PO}_{4}, 2 \mathrm{mmol} / \mathrm{MgCl}$, $3 \mathrm{mmol} / \mathrm{lmalic}$ acid and $0.1 \%$ [wt/vol.] fatty acid-free BSA, $\mathrm{pH} 7.2)$ fuelled by succinate $(10 \mathrm{mmol} / \mathrm{l})$ in the presence of complex III inhibitor antimycin $(1 \mu \mathrm{mol} / \mathrm{l})$ to maximise ROS production [27]. The reaction started upon the addition of Amplex Red reagent $(100 \mu \mathrm{mol} / \mathrm{l})$ and horseradish peroxidase $(2 \mathrm{U} / \mathrm{ml})$. Superoxide dismutase $(100 \mathrm{U} / \mathrm{ml})$ was added to the buffer to ensure complete conversion of superoxide to $\mathrm{H}_{2} \mathrm{O}_{2}$ and to prevent the superoxide radical from interacting with the horseradish peroxidase [28]. 


\section{Statistics}

Results are expressed as means \pm SEM and were analysed by one-way ANOVA followed by Newman-Keuls post-hoc test, or by two-way ANOVA followed by Bonferroni's post-hoc test where appropriate. Basal and insulin-stimulated conditions in muscle were analysed by twotailed Student's $t$ test. Significance was set at $p<0.05$. All graphs and statistical analyses were performed using GraphPad Prism 5.0 (GraphPad Software, San Diego, CA, USA).

\section{RESULTS}

\section{SkQ treatment does not improve palmitate-induced insulin resistance in vitro}

SkQ treatment significantly lowered oxidative stress by $23 \%(p<0.05)$ (Figure 1a, b) as determined by protein carbonyl content, a marker of oxidative damage, in $\mathrm{C} 2 \mathrm{C} 12$ myotubes under basal conditions. We investigated whether this reduced level of oxidative stress affected cellular glucose uptake under basal, insulin-stimulated and palmitate-induced insulin-resistant conditions. Basal glucose uptake was similar between control and SkQtreated myotubes (control 1.00 \pm 0.09 , SkQ $0.94 \pm 0.06$ arbitrary units $[A U], n=5-7, p=N S$ ). Insulin-stimulated glucose uptake was comparable between control and SkQ-treated myotubes (Figure 1c). The incubation of $\mathrm{C} 2 \mathrm{C} 12$ muscle cells with palmitate induced insulin resistance, as seen in the significant reduction of glucose uptake upon insulin stimulation in control and SkQ-treated cells $(p<0.001$, two-way ANOVA). There was no significant difference between palmitate-induced insulin resistance in control and SkQ-treated myotubes (Figure 1c). Furthermore, pAkt, a marker of insulin signalling, revealed comparable results. Basal pAkt levels (control 0.22 \pm 0.06 , SkQ $0.18 \pm 0.04 \mathrm{AU}, \mathrm{n}=8, \mathrm{p}=\mathrm{NS}$ ) and insulin induction of pAkt levels were similar in both cell groups (Figure 1d). Palmitate also reduced the insulinstimulated pAkt response by about half in control myotubes, a reduction not restored by SkQ treatment ( $<<0.001$, two-way ANOVA) (Figure 1d). No significant difference was seen in pAkt levels between control and SkQ myotubes in the presence of palmitate. Total Akt levels were comparable between control and SkQ myotubes under all test conditions (Figure 1d). Thus the SkQ-induced lowering of oxidative stress did not suffice to ameliorate lipid-induced insulin resistance in muscle cells.

\section{SkQ treatment in vivo does not influence weight gain or plasma lipid levels}

To extend our findings from the cell model to the in vivo situation, we examined the effects of SkQ treatment in mice. Male mice (8 weeks old) were maintained on an LF or placed on an HF for 16 weeks. A second group of HF-fed mice received drinking water supplemented with SkQ $(H F+S k Q)$. HF mice weighed $31 \%(p<0.0001)$ more than LF mice and had significantly elevated NEFA, glucose and insulin levels (Table 1). The HF+SkQ group also had 


\section{CHAPTER 2}

significantly more weight gain and elevated plasma values compared with LF controls, with changes similar to those observed in HF mice. Food intake was comparable in all three groups (Table 1), suggesting no taste aversion to the SkQ treatment and normal behaviour.

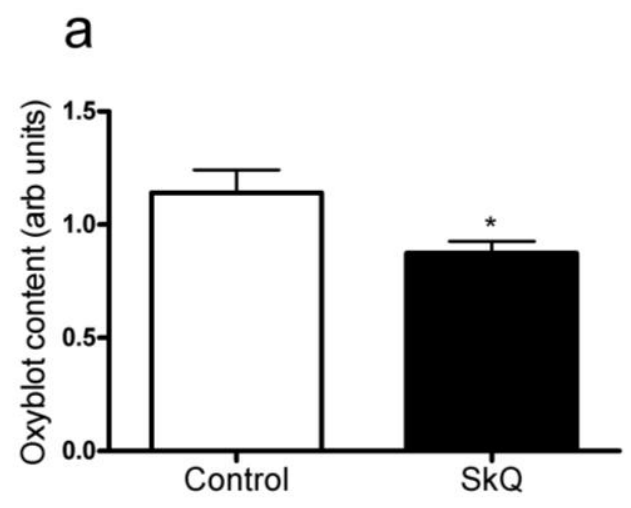

b
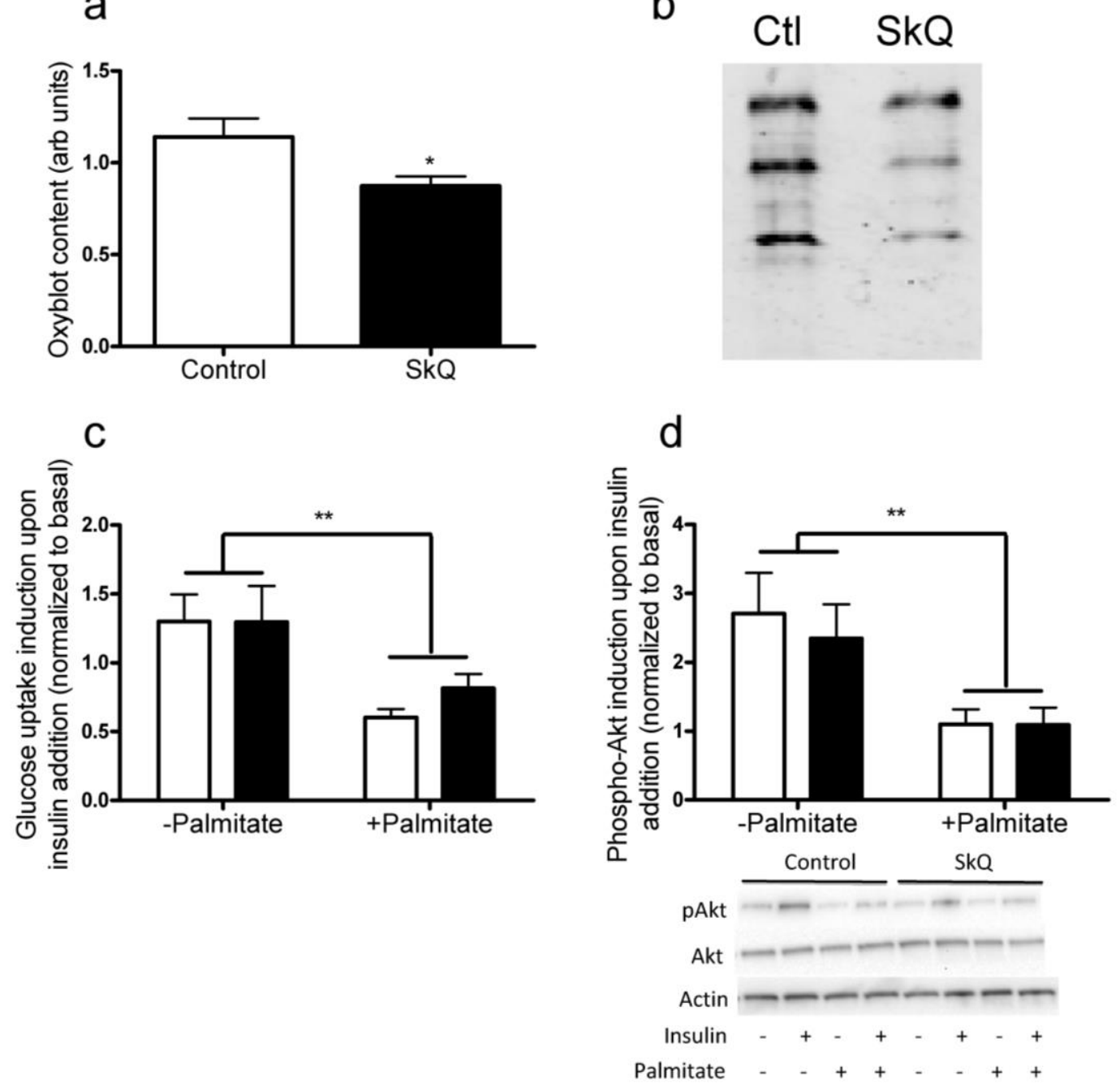

Figure 1. ROS sequestering and insulin sensitivity in $\mathrm{C} 2 \mathrm{C} 12$ cells. (a) Oxyblot content and (b) representative blot in differentiated $\mathrm{C} 2 \mathrm{C} 12$ cells without (control [Ctrl]) or with SkQ treatment; $n=6$ per condition. Glucose uptake (c) in control (white bars) and SkQ-pretreated (black bars) cells upon insulin stimulation in the absence or presence of palmitate conditions as indicated; $n=5-8$ per condition. (d) pAkt abundance under the same conditions as above (c), with representative blot of pAkt, total Akt and actin (loading control) under basal, and insulin- and palmitate-stimulated conditions. Data (a, c, d) are presented as mean \pm SEM; * $p<0.05$ and ${ }^{* *} p<0.01$

\section{SkQ treatment reduces HF-induced oxidative damage}

Oxidative stress measured as protein carbonyl content was increased by $56 \%$ upon HF feeding ( $p<0.05$ vs LF) (Figure $2 \mathrm{a}, \mathrm{b}$ ). Consistent with our findings in $\mathrm{C} 2 \mathrm{C} 12$ muscle cells, $\mathrm{SkQ}$ treatment in vivo effectively prevented the diet-induced increase in skeletal muscle oxidative damage ( $p<0.5$ vs HF) (Figure $2 a, b)$. Since SkQ specifically targets mitochondrial ROS production, we also measured $\mathrm{H}_{2} \mathrm{O}_{2}$ release from freshly isolated skeletal muscle 
mitochondria. $\mathrm{H}_{2} \mathrm{O}_{2}$ emission was significantly increased in the $\mathrm{HF}$ group compared with $\mathrm{LF}$ (54\%, $\mathrm{p}<0.05$ ) (Figure $2 \mathrm{c}$ ). SkQ treatment normalised $\mathrm{H}_{2} \mathrm{O}_{2}$ levels in $\mathrm{HF}+\mathrm{SkQ}$ mice to those of the LF controls (Figure 2c), thus confirming the efficacy of the mitochondrial-specific ROS scavenger as shown above and previously reported [12-14]. In addition, SOD2 (the mitochondrial SOD) activity was significantly elevated in HF mice (twofold vs LF, $p<0.01$ ) (Figure 2d), suggesting a state of increased ROS production. SkQ treatment significantly blunted the HF-induced increase in SOD2 activity $(p<0.05)$ (Figure $2 d)$. Finally, a trend was observed for increased activity of the cytosolic antioxidant GPx1 in both HF-fed groups (Table 1).

Table 1: Body weight, plasma values and skeletal muscle enzyme activity

\begin{tabular}{|c|c|c|c|c|}
\hline Variable & LF & HF & HF+SkQ & $p$ value \\
\hline Body weight (g) & $27.6 \pm 0.4$ & $40.1 \pm 1.2^{\star \star *}$ & $40.3 \pm 1.3^{\star \star \star}$ & $<0.0001$ \\
\hline Food intake (kJ/week) & $489.9 \pm 19.2$ & $494.1 \pm 34.7$ & $584.9 \pm 64.0$ & NS \\
\hline Triacylglycerol (mmol/l) & $1.99 \pm 0.30$ & $2.67 \pm 0.12$ & $2.94 \pm 0.34$ & 0.0735 \\
\hline $\operatorname{NEFA}(\mathrm{mmol} / \mathrm{l})$ & $0.53 \pm 0.09$ & $1.24 \pm 0.15^{\star \star \star}$ & $1.30 \pm 0.06^{\star * *}$ & 0.0003 \\
\hline Glucose (mmol/l) & $5.87 \pm 0.25$ & $7.98 \pm 0.69^{* \star}$ & $8.13 \pm 0.33^{\star \star}$ & 0.0027 \\
\hline Insulin (pmol/l) & $61.2 \pm 14.3$ & $218.7 \pm 28.9^{\star \star \star}$ & $212.1 \pm 20.5^{\star \star \star}$ & 0.0003 \\
\hline GPx1 activity (mU/mg) & $5.83 \pm 0.37$ & $7.01 \pm 0.37$ & $6.65 \pm 0.24$ & 0.07 \\
\hline HADH activity(U/mg) & $38.54 \pm 6.57$ & $62.07 \pm 8.31$ & $63.47 \pm 9.37$ & 0.07 \\
\hline
\end{tabular}

Values are expressed as mean \pm SEM; $n=5-10 ;{ }^{*} p<0.05,{ }^{* *} p<0.01$ and ${ }^{* * *} p<0.001$ vs LF

\section{ROS sequestering does not improve insulin sensitivity}

Both $\mathrm{HF}$ and $\mathrm{HF}+\mathrm{SkQ}$ mice displayed exacerbated glucose metabolism compared with LF mice, as illustrated by decreased glucose tolerance after 15 weeks of the intervention (Figure 3a). The AUC for glucose levels during the glucose tolerance test was significantly increased by $33 \%(p<0.001)$ in HF and by $22 \%(p<0.01)$ in HF+SkQ mice (Figure $3 b)$. Insulin levels measured during the glucose tolerance test were also significantly higher in both groups (Figure 3c). Insulin AUC levels were increased twofold $(p<0.05)$ and fourfold $(p<0.01)$ in HF and $\mathrm{HF}+\mathrm{SkQ}$, respectively, compared with LF mice (Figure $3 \mathrm{~d}$ ), indicative of the hyperinsulinaemia associated with insulin resistance. No statistical difference between HF and $\mathrm{HF}+\mathrm{SkQ}$ mice was observed for any of the variables mentioned above. In addition, muscle-specific insulin signalling was evaluated by examining levels of pAkt and its downstream target, phosphorylated GSK3 (pGSK3), in gastrocnemius muscle under basal and insulin-stimulated conditions (Figure 4). Although insulin significantly stimulated pAkt levels in LF, HF and HF+SkQ mice ( $p<0.001$, basal vs insulin) (Figure $4 a$ ), the absolute increase in insulin-stimulated pAkt was blunted in $\mathrm{HF}$ and $\mathrm{HF}+\mathrm{SkQ}$ mice $(\mathrm{HF}-35 \%, \mathrm{p}<0.01$; 


\section{CHAPTER 2}

$H F+S k Q-62 \%, p<0.001$ compared with LF). In addition, pGSK3 was significantly increased upon insulin stimulation in LF mice $(74 \%$ vs basal, $p<0.05)$ (Figure $4 b)$, while insulin signalling was severely disrupted in $\mathrm{HF}$ and $\mathrm{HF}+\mathrm{SkQ}$ mice, as there was no insulin-mediated increase in pGSK3 over basal levels ( $p=N S$ ) (Figure $4 b$ ). Total Akt was similar for all three groups under basal and insulin-stimulated conditions (Figure 4a); moreover, no change was observed in IRS1 protein abundance (Figure 4c). Phosphorylation levels of JNK, an inflammation marker of insulin resistance, were unaltered in all three groups (Figure 4d), suggesting that $\mathrm{HF}$ and $\mathrm{HF}+\mathrm{SkQ}$ mice have disruptions in insulin signalling, with no effect on inflammatory stress signalling.

a

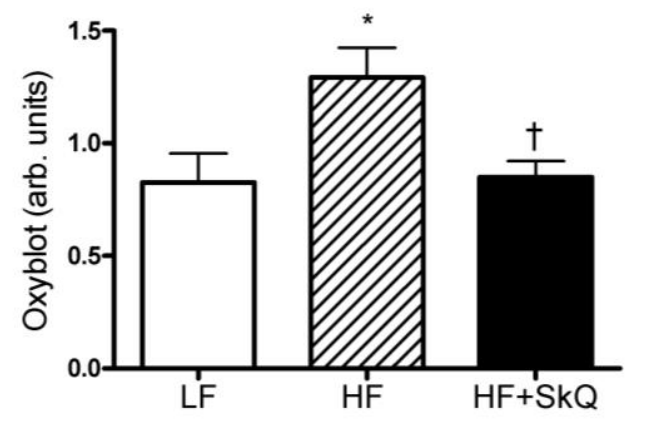

C

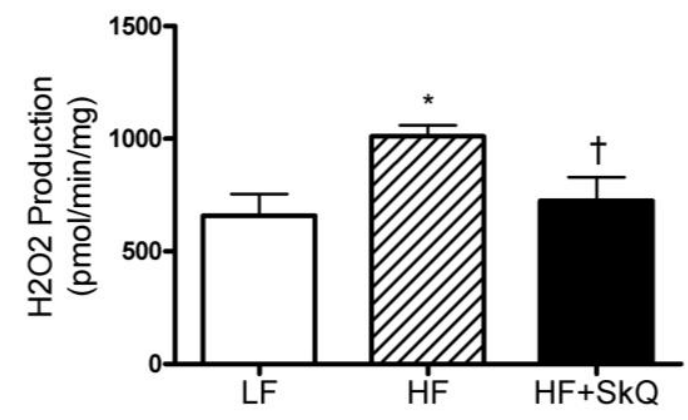

b L $\underline{\text { HF }} \underline{\mathrm{HF}+\mathrm{SkQ}}$

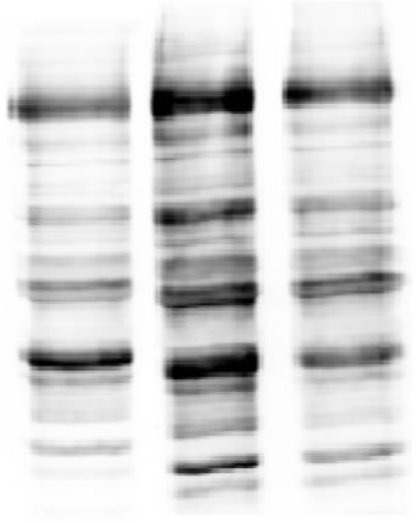

d

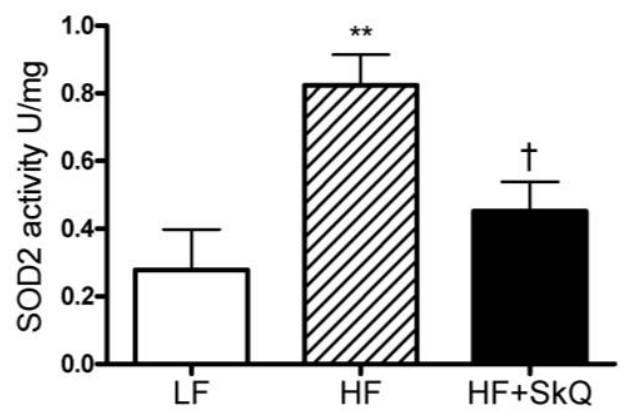

Figure 2. SkQ treatment effectively blunts HF-induced oxidative stress and ROS production. (a) Quantification of muscle protein carbonyl in LF, HF and HF+SkQ mice, with (b) a representative protein Oxyblot. (c) Isolated mitochondria hydrogen peroxide production and (d) SOD2 activity assay in muscle homogenates. Data are presented as mean \pm SEM; $n=7$ per group for isolated mitochondrial assays and $n=5$ per group for activity and oxyblot assays; ${ }^{*} p<0.05$ vs LF, ${ }^{* *} p<0.01$ vs LF and ${ }^{\dagger} p<0.05$ vs HF 

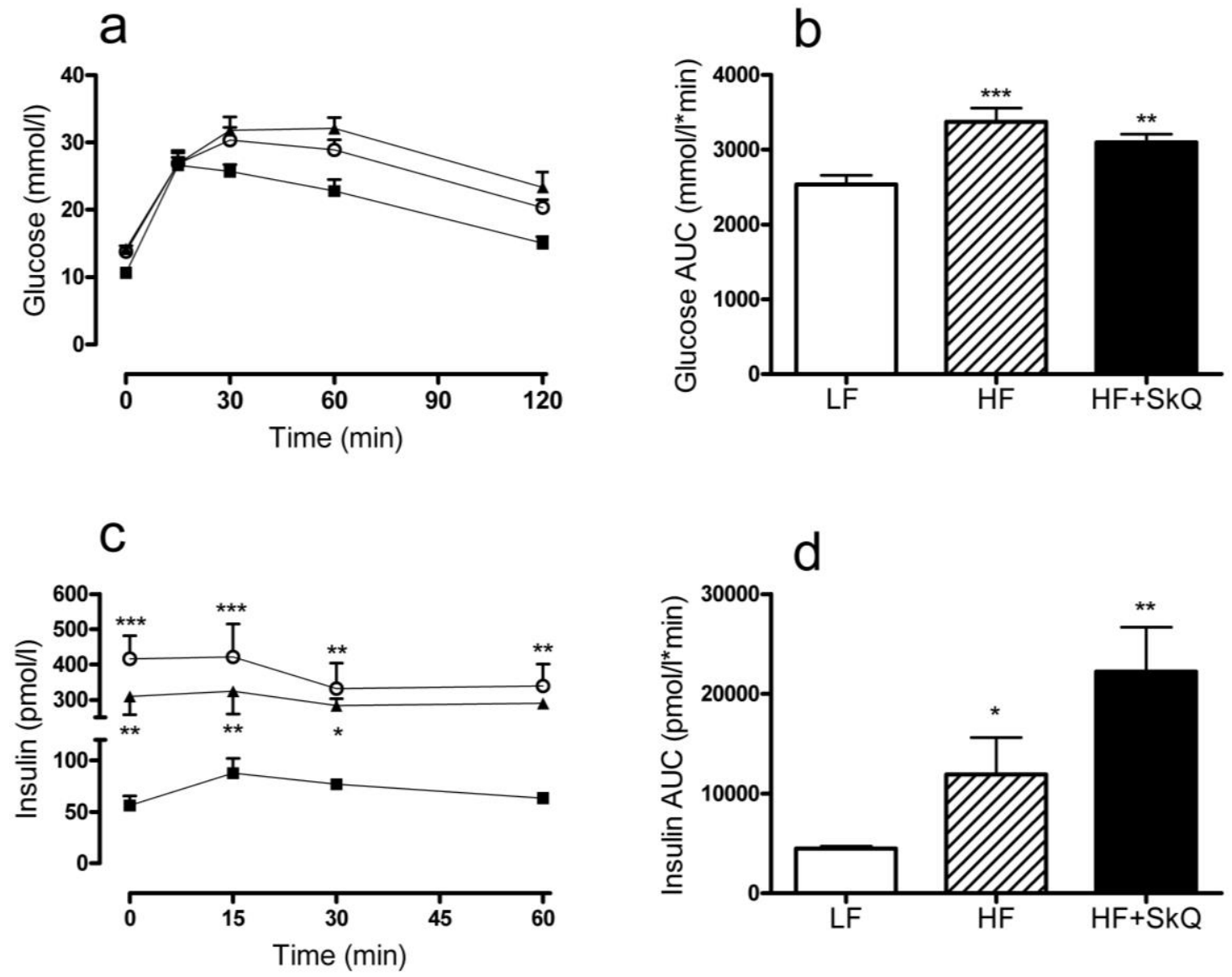

Figure 3. ROS sequestering does not improve glucose tolerance in HF-fed mice. (a) Glucose tolerance curves after 15 weeks of intervention in LF- (black squares), HF- (black triangles) and HF+SkQ-fed (white circles) mice, with (b) AUC analysis in groups as indicated. (c) Insulin levels and (d) AUC during the glucose tolerance test in the same groups. Data are presented as mean \pm SEM; $n=6-10$ mice per group; ${ }^{*} p<0.05,{ }^{* *} p<0.01$ and ${ }^{* * *} p<0.001$ vs LF. Two-way ANOVA analysis revealed group $(p<0.0001)$ and time $(p<0.0001)$ effects for glucose, and a group $(p<0.0001)$ effect for insulin during the glucose tolerance test

\section{The effect of ROS sequestering on mitochondrial content and respiration}

Mitochondrial density was estimated by mitochondrial DNA copy number, CS activity and the protein content of structural components of the respiratory chain complexes (OXPHOS). Whereas no significant difference was detected in the mitochondria DNA copy number (Figure 5a), CS activity was significantly increased in HF mice $(65 \%, p<0.01)$ (Figure 5b) compared with LF. Similarly, the OXPHOS complexes were increased in HF mice $(57 \%$ vs LF, $p<0.01$ ) (Figure 5c, d). Interestingly, these HF-induced increases in CS activity and OXPHOS content were normalised to LF levels in HF+SkQ mice (Figure 5b, c). Finally, we investigated the impact of ROS sequestering on mitochondrial respiration, fuelled by either a carbohydrate-derived (pyruvate) or a fatty acid-derived (palmitoyl-CoA in the presence of carnitine) substrate in isolated mitochondria (Table 2). Under the HF condition, ADPstimulated (state 3$)$ pyruvate-supported respiration rates tended to decrease $(p=0.06)$ compared with LF mice. The maximum carbonyl cyanide $p$-trifluoromethoxyphenylhydrazone 
(FCCP)-induced (state $U$ ) respiration, indicating the maximum capacity of the electron transport chain on a given substrate, was significantly decreased by $30 \%$ vs LF mice $(p<0.05)$ (Table 2). At the same time, there was no change in the oligomycin-insensitive (state 4) respiration rate, a marker of mitochondrial proton leak. SkQ supplementation did not restore the HF-induced reduction in pyruvate-supported respiration, as $\mathrm{HF}+\mathrm{SkQ}$ mice displayed similar decreases in pyruvate-supported state 3 and state $U(p<0.05)$ respiration rates. We did not observe any change in fatty acid-supported mitochondrial respiration under ADP- or maximal-stimulated conditions in any of the groups. Furthermore, skeletal muscle HADH activity (a beta-oxidation enzyme) showed a tendency to be upregulated under both high-fat conditions (Table 1).
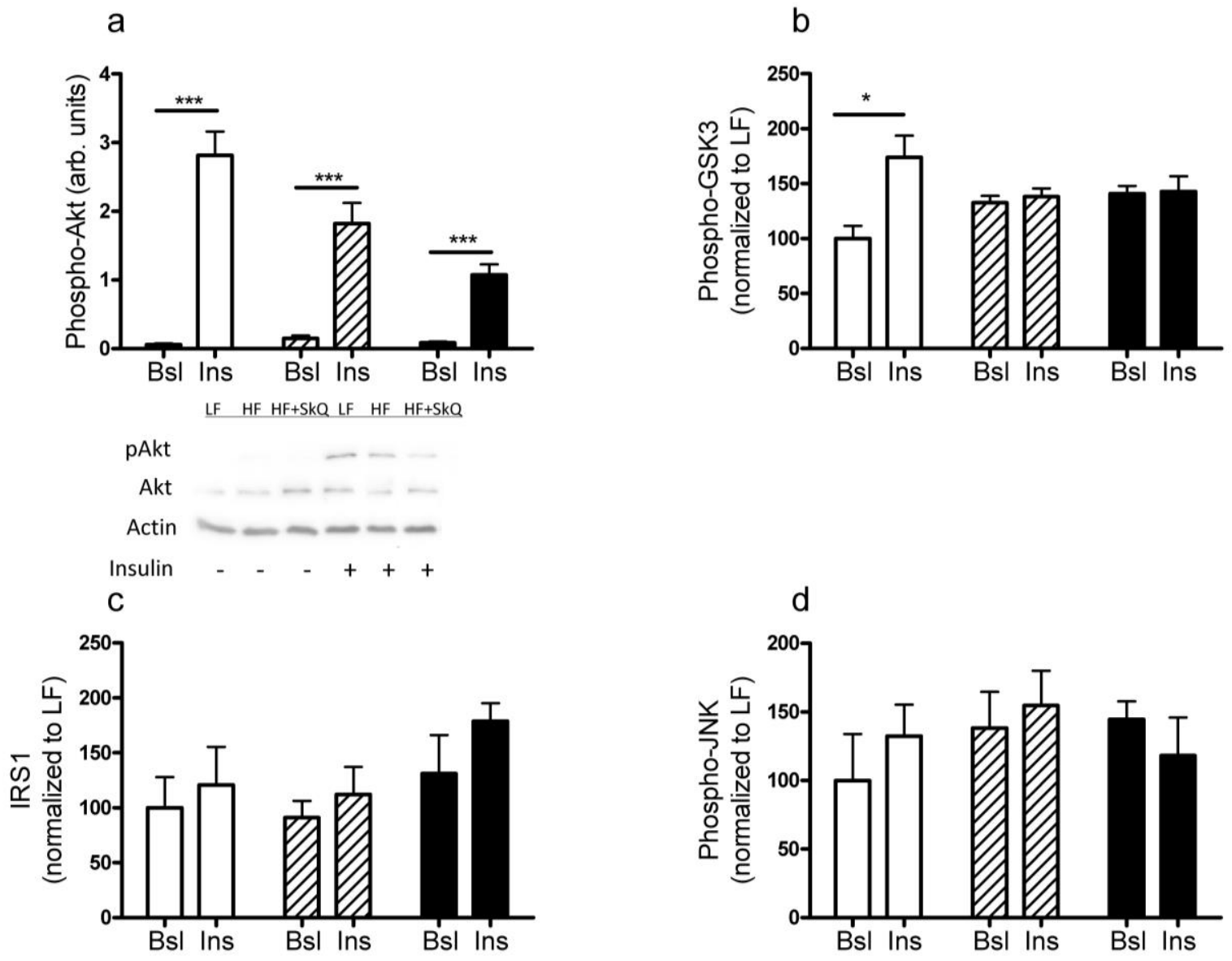

Figure 4. Skeletal muscle insulin signalling. (a) Basal saline control (BsI) and insulin-stimulated (Ins) pAkt in LF (white bars), $\mathrm{HF}$ (hatched bars) and $\mathrm{HF}+\mathrm{SkQ}$ (black bars) gastrocnemius samples, with representative pAkt, total Akt and actin (loading control) blots. (b) Levels of pGSK3, (c) total IRS1 content and (d) pJNK in groups as above (a). Data $(\mathbf{a}, \mathbf{c}-\mathbf{e})$ are presented as mean \pm SEM; $n=5$ mice per group per condition; ${ }^{*} p<0.05$ and ${ }^{* * *} p<0.001$ for basal vs insulin-stimulated conditions 
a

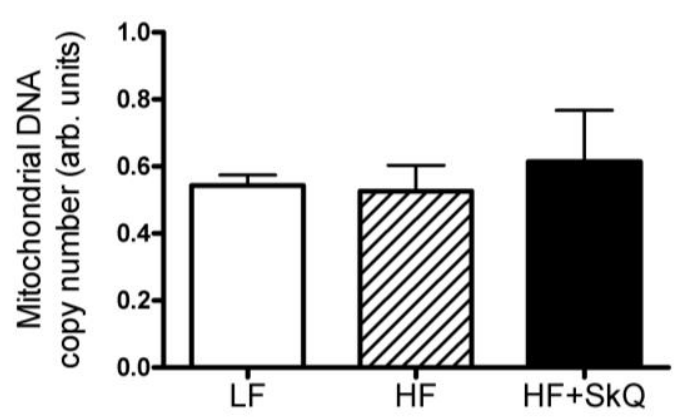

C

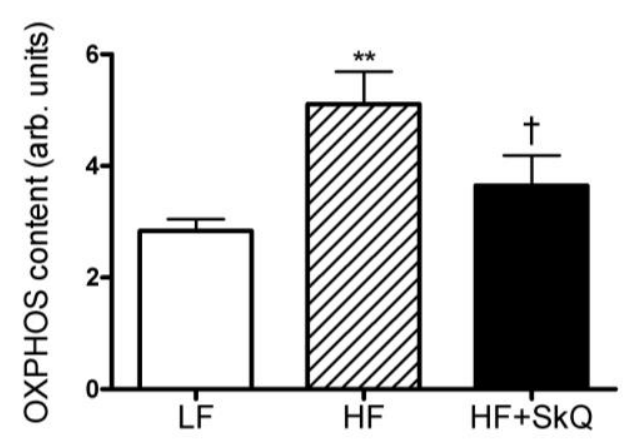

b

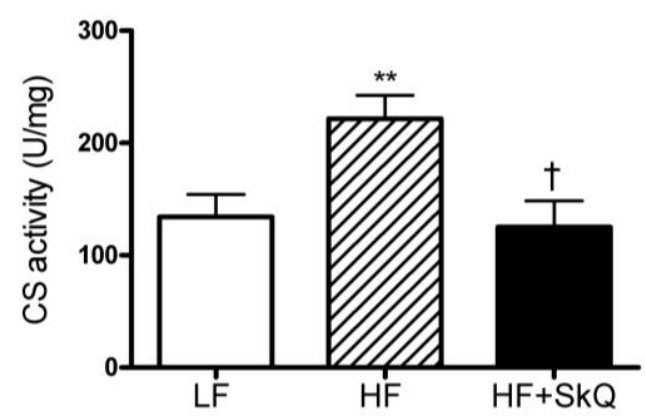

d

LF HF HF+SkQ

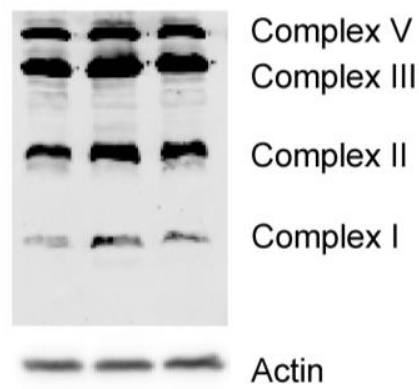

Figure 5. Mitochondrial density markers. (a) Mitochondrial DNA copy number, (b) gastrocnemius muscle CS activity, (c) total OXPHOS complex quantification and (d) representative blots with sarcomeric actin as loading control for mouse groups as shown. Data are presented as mean $\pm S E M ; n=5$ for mtDNA and western blot and $n=10$ for CS assay; ${ }^{* *} p<0.01$ vs LF and ${ }^{\dagger} p<0.05$ vs HF

Table 2: Intrinsic mitochondrial respiration levels

\begin{tabular}{lcccc} 
Table 2: Intrinsic mitochondrial respiration levels & \multirow{2}{c}{ Respiration pmol $(\mathbf{s \times m g})^{-1}$} & \\
\cline { 2 - 4 } Respiration state & LF & HF & HF+SkQ & p value \\
\cline { 2 - 4 } $\begin{array}{l}\text { Pyruvate } \\
\text { State 3 }\end{array}$ & & & \\
State 4 & $4456 \pm 441.3$ & $3928 \pm 515.1$ & $3680 \pm 481.2$ & 0.0621 \\
State U & $9188 \pm 650.8$ & $6348 \pm 886.8^{*}$ & $6436 \pm 771.3^{*}$ & 0.0291 \\
Palmitoyl CoA+carnitine & & & & \\
State 3 & $1413 \pm 341.4$ & $1348 \pm 212.5$ & $1467 \pm 244.9$ & NS \\
State 4 & $446.9 \pm 40.1$ & $392.2 \pm 40.5$ & $395.0 \pm 36.5$ & NS \\
State U & $1781 \pm 472.4$ & $1654 \pm 285.5$ & $1859 \pm 331.8$ & NS
\end{tabular}

Values expressed as mean $\pm S E M ; n=6-7$. ADP-stimulated (state 3 ) respiration was induced by the addition of ADP $(450 \mu \mathrm{mol} / \mathrm{l})$, state 4 respiration was attained upon addition of the ATP-synthase inhibitor oligomycin (1 $\mu \mathrm{g} / \mathrm{ml}$ ) and maximum oxygen flux (state uncoupled) was achieved by titrating the chemical uncoupler FCCP ${ }^{*} p<0.05$ vs LF 


\section{DISCUSSION}

In the present study, we tested whether mitochondria-targeted ROS sequestering (SkQ treatment) was able to preserve skeletal muscle insulin sensitivity in a muscle cell model of fatty acid-induced and in a mouse model of diet-induced insulin resistance. In vitro, SkQ treatment effectively reduced oxidative stress in $\mathrm{C} 2 \mathrm{C} 12$ myotubes, but did not improve glucose uptake or insulin signalling under lipid-induced insulin resistance. In vivo, SkQ treatment successfully normalised the level of HF-induced oxidative stress in mouse muscle, but this did not reverse the reduction in glucose tolerance and skeletal muscle insulin sensitivity upon HF feeding.

Several studies have shown a link between insulin resistance, ROS production and oxidative stress in in vitro and in vivo models. Palmitate exposure in L6 myotubes resulted in a dose-dependent decrease in insulin-mediated glucose uptake, paralleled by increased superoxide production [29] and mitochondrial DNA damage [30]. In addition, cultured cells incubated with $\mathrm{H}_{2} \mathrm{O}_{2}$ had greater insulin resistance [31], and ROS production was identified as the common denominator and causal factor in several cellular models of insulin resistance [4]. Many studies have also reported beneficial effects on insulin sensitivity when oxidative stress was blunted. As mentioned previously, MCAT overabundance in mice improved insulin sensitivity and attenuated $\mathrm{H}_{2} \mathrm{O}_{2}$ emission in [5] ageing- and [1] high-fat diet-induced models of insulin resistance. Similarly, rats treated with a small mitochondrial antioxidant peptide were protected from high-fat diet-induced mitochondrial $\mathrm{H}_{2} \mathrm{O}_{2}$ emission, impaired glucose uptake and reduced skeletal muscle insulin sensitivity [1]. In contrast to these studies [1,5], we were unable to confirm that interfering with mitochondrial ROS production, which leads to reduced oxidative stress levels, averts HF-induced insulin resistance in skeletal muscle. These discrepancies may be due to the technical approach used (genetic overexpression vs oral antioxidant), the induction of oxidative stress (age- vs diet-induced) and/or the duration of the intervention (6 vs 16 weeks). In addition, the diet composition may also have led to differences between the studies. In the present study, we used a diet high in fat and sucrose, which is known to induce oxidative stress, mitochondrial dysfunction and insulin resistance [32]. This may be relevant since the source of fat has been implicated as a major contributor to lipid-induced ROS and insulin resistance [30], but high sucrose levels also increase ROS production and oxidative stress [33].

However, not all studies support the notion that ROS production is a causal factor in insulin resistance. Thus it has been shown that insulin stimulates the generation of ROS, since when insulin binds to its receptor, a short burst of cellular ROS is produced, acting as second messenger that mediates insulin signalling [34, 35]. Furthermore, Loh et al [36] demonstrated that subtle increases in ROS production were in fact associated with improved 
insulin sensitivity in mice deficient in the cytosolic antioxidant GPx1, suggesting that ROS may even help enhance insulin sensitivity. At the same time, overabundance of GPx1 resulted in hyperglycaemia, hyperinsulinaemia and insulin resistance in mice [37], indicating that $\mathrm{H}_{2} \mathrm{O}_{2}$ quenching disrupts insulin action. Moreover, Yokota et al [38] demonstrated that mice fed a high-fat diet and treated with apocynin, an $\mathrm{NAD}(\mathrm{P}) \mathrm{H}$ oxidase inhibitor, had blunted skeletal muscle superoxide production, yet with no effect on glucose uptake or other markers of insulin sensitivity. Finally, Abdul-Ghani et al [39] failed to show a difference in skeletal muscle mitochondrial $\mathrm{H}_{2} \mathrm{O}_{2}$ levels between type 2 diabetic participants and age-matched controls, suggesting that increased mitochondrial ROS is not a mediator of insulin resistance. Here, we demonstrated, using a novel mitochondria-specific antioxidant, that in vitro reductions of oxidative stress did not avert lipid-induced disruptions of insulin-stimulated glucose uptake and insulin signalling, although it remains possible that palmitate exposure in these cells leads to insulin resistance via mechanisms that are independent of oxidative stress. On the other hand, in vivo whole-body glucose tolerance, as well as insulin-mediated pAkt in skeletal muscle remained impaired in mice on an HF supplemented with $S k Q$, a mitochondria-targeted antioxidant, despite reduced skeletal muscle oxidative stress. Although the slightly increased insulin levels during the glucose tolerance test in our HF+SkQ mice suggest that $\mathrm{SkQ}$ treatment causes an even higher degree of HF-induced insulin resistance, this is not supported by the results for muscle pAkt and in vitro glucose uptake. However, as we did not perform clamp studies, it is possible that effects on skeletal muscle insulin-stimulated glucose uptake in vivo remained undetected. Moreover, alterations in muscle glucose transport subsequent to lipid-induced insulin resistance may exist, regardless of an effect on Akt signalling [40].

Bonnard et al [32] demonstrated that when mice were fed a high-fat high-sucrose diet, similar to that used in the present study, oxidative stress was coupled to reduced mitochondrial biogenesis and dysfunction. In contrast, several studies have observed upregulated mitochondria biogenesis in response to a high-fat insult [41, 42]; however, this response seems to be dependent on the intervention time [21,25] and/or the method of determining mitochondrial density [32]. In the present study, we measured several markers of mitochondrial density, and observed that OXPHOS content and CS activity revealed increased mitochondrial density in the HF-fed condition, in agreement with previous reports [41]. Interestingly, these two markers were normalised to LF levels upon ROS sequestering. This finding is in line with other reports showing that antioxidant supplementation suppresses exercise-induced mitochondrial biogenesis [43,44], suggesting a potential role of ROS in mitochondrial biogenesis, although not all studies support this conclusion [45].

We also observed a substrate-specific reduction upon pyruvate (plus malate)supported mitochondrial respiration. These results are consistent with those of Mogensen et 
al [46], who also demonstrated reduced pyruvate-supported respiration rates with no change in lipid-supported respiration rates in isolated muscle mitochondria from type 2 diabetes participants compared with age- and BMI-matched controls. Thus SkQ treatment effectively reduced skeletal muscle oxidative damage, but did not reverse the HF-induced decrease in pyruvate-supported respiration. Therefore, we conclude that this reduction in pyruvatesupported mitochondrial respiration does not occur as a result of oxidative stress. It is tempting to speculate that increased markers of mitochondrial content (OXPHOS proteins and CS activity) in HF-fed mice may have compensated for the reduced intrinsic function. Since this response appeared to be blunted in $\mathrm{HF}+\mathrm{SkQ}$ mice, the latter are likely to have had lower total mitochondrial oxidative capacity than HF-fed mice, despite similar levels of insulin resistance. These findings also argue against mitochondrial dysfunction playing a causal role in the development of HF-induced insulin resistance, which has been suggested previously $[6,7]$.

A potential limitation of the present study is that only the 16 week endpoint variables were examined, making it impossible to comment on the favourable short-term effects of antioxidant treatment on insulin sensitivity that have been reported by others [47]. Nor can we exclude putative beneficial effects of SkQ treatment upon prolonged exposure. Furthermore, we only tested one dose. In this respect, other studies have clearly shown the dose used by us to be optimal in inbred and outbred rodents in longevity studies [16, 48]. However, it is possible that the full blockade of HF-induced ROS production was too rigid, dissipating putative beneficial effects of ROS, such as stimulation of endogenous antioxidant capacity or mitochondrial biogenesis, which themselves might in turn affect insulin sensitivity. Finally, oxidative stress is one of several putative mechanisms associated with lipid-induced insulin resistance. If lipid-induced insulin resistance is independent of increased ROS production, then an intervention designed to suppress oxidative stress clearly will not improve insulin sensitivity.

In conclusion, an antioxidant specific to the sequestering of mitochondrial ROS production (SkQ) successfully suppressed oxidative damage in vitro and under HF conditions in vivo. Despite this relief of oxidative stress, SkQ treatment did not ameliorate lipid-induced insulin resistance in the two study models. Therefore, our results suggest that diet-induced oxidative stress is not a prerequisite for the development of skeletal muscle insulin resistance.

\section{Acknowledgements}

The authors would like to thank O. Fedorkin from Mitotech (Moscow, Russia) and V.P Skulachev (Lomonosov Moscow State University, Moscow, Russia) for donating the SkQ compound and reviewing the manuscript. 


\section{Funding}

This research was supported by a grant from the European Foundation for the Study of Diabetes. P. Schrauwen is supported by a $\mathrm{VICl}$ research grant for innovative research from the Netherlands Organization for Scientific Research (Grant 918.96.618).

\section{REFERENCES}

1. Anderson, E.J., et al., Mitochondrial $\mathrm{H} 2 \mathrm{O} 2$ emission and cellular redox state link excess fat intake to insulin resistance in both rodents and humans. J Clin Invest, 2009. 119: p. 573-81.

2. Lefort, N., et al., Increased reactive oxygen species production and lower abundance of complex I subunits and carnitine palmitoyltransferase 1B protein despite normal mitochondrial respiration in insulin-resistant human skeletal muscle. Diabetes, 2010. 59(10): p. 2444-52.

3. Haber, C.A., et al., N-acetylcysteine and taurine prevent hyperglycemia-induced insulin resistance in vivo: possible role of oxidative stress. Am J Physiol Endocrinol Metab, 2003. 285(4): p. E744-53.

4. Houstis, N., E.D. Rosen, and E.S. Lander, Reactive oxygen species have a causal role in multiple forms of insulin resistance. Nature, 2006. 440(7086): p. 944-8.

5. Lee, H.Y., et al., Targeted expression of catalase to mitochondria prevents ageassociated reductions in mitochondrial function and insulin resistance. Cell Metab, 2010. 12(6): p. 668-74.

6. Kelley, D.E., et al., Dysfunction of mitochondria in human skeletal muscle in type 2 diabetes. Diabetes, 2002. 51(10): p. 2944-50.

7. Petersen, K.F., et al., Impaired mitochondrial activity in the insulin-resistant offspring of patients with type 2 diabetes. N Engl J Med, 2004. 350(7): p. 664-71.

8. Schrauwen-Hinderling, V.B., et al., Impaired in vivo mitochondrial function but similar intramyocellular lipid content in patients with type 2 diabetes mellitus and BMImatched control subjects. Diabetologia, 2007. 50(1): p. 113-20.

9. Heilbronn, L.K., et al., Markers of mitochondrial biogenesis and metabolism are lower in overweight and obese insulin-resistant subjects. J Clin Endocrinol Metab, 2007. 92(4): p. 1467-73.

10. Szendroedi, J., et al., Muscle mitochondrial ATP synthesis and glucose transport/phosphorylation in type 2 diabetes. PLoS Med, 2007. 4(5): p. e154.

11. Phielix, E., et al., Lower intrinsic ADP-stimulated mitochondrial respiration underlies in vivo mitochondrial dysfunction in muscle of male type 2 diabetic patients. Diabetes, 2008. 57(11): p. 2943-9.

12. Skulachev, V.P., et al., An attempt to prevent senescence: a mitochondrial approach. Biochim Biophys Acta, 2009. 1787(5): p. 437-61.

13. Antonenko, Y.N., et al., Mitochondria-targeted plastoquinone derivatives as tools to interrupt execution of the aging program. 1. Cationic plastoquinone derivatives: synthesis and in vitro studies. Biochemistry (Mosc), 2008. 73(12): p. 1273-87.

14. Bakeeva, L.E., et al., Mitochondria-targeted plastoquinone derivatives as tools to interrupt execution of the aging program. 2. Treatment of some ROS- and age-related diseases (heart arrhythmia, heart infarctions, kidney ischemia, and stroke). Biochemistry (Mosc), 2008. 73(12): p. 1288-99.

15. Hommelberg, P.P., et al., Palmitate-induced skeletal muscle insulin resistance does not require NF-kappaB activation. Cell Mol Life Sci, 2011. 68(7): p. 1215-25.

16. Neroev, V.V., et al., Mitochondria-targeted plastoquinone derivatives as tools to interrupt execution of the aging program. 4. Age-related eye disease. SkQ1 returns vision to blind animals. Biochemistry (Mosc), 2008. 73(12): p. 1317-28.

17. Anisimov, V.N., et al., Mitochondria-targeted plastoquinone derivatives as tools to interrupt execution of the aging program. 5. SkQ1 prolongs lifespan and prevents development of traits of senescence. Biochemistry (Mosc), 2008. 73(12): p. 1329-42. 
18. Paglialunga, S., et al., Reduced adipose tissue triglyceride synthesis and increased muscle fatty acid oxidation in C5L2 knockout mice. J Endocrinol, 2007. 194(2): p. 293-304.

19. Ouwens, D.M., et al., A mutant insulin receptor induces formation of a Shc-growth factor receptor bound protein 2 (Grb2) complex and p21ras-GTP without detectable interaction of insulin receptor substrate 1 (IRS1) with Grb2. Evidence for IRS1independent p21ras-GTP formation. J Biol Chem, 1994. 269(52): p. 33116-22.

20. Lenaers, E., et al., Adaptations in mitochondrial function parallel, but fail to rescue, the transition to severe hyperglycemia and hyperinsulinemia: a study in Zucker diabetic fatty rats. Obesity (Silver Spring), 2010. 18(6): p. 1100-7.

21. Sparks, L.M., et al., A high-fat diet coordinately downregulates genes required for mitochondrial oxidative phosphorylation in skeletal muscle. Diabetes, 2005. 54(7): p. 1926-33.

22. Oberley, L.W. and D.R. Spitz, Assay of superoxide dismutase activity in tumor tissue. Methods Enzymol, 1984. 105: p. 457-64.

23. Paglia, D.E. and W.N. Valentine, Studies on the quantitative and qualitative characterization of erythrocyte glutathione peroxidase. J Lab Clin Med, 1967. 70(1): p. 158-69.

24. Hoeks, J., et al., Enhanced lipid -but not carbohydrate- supported mitochondrial respiration in skeletal muscle of PGC-1alpha overexpressing mice. J Cell Physiol, 2011.

25. Hoeks, J., et al., Mitochondrial function, content and ROS production in rat skeletal muscle: effect of high-fat feeding. FEBS Lett, 2008. 582(4): p. 510-6.

26. Shabalina, I.G., et al., Cold tolerance of UCP1-ablated mice: a skeletal muscle mitochondria switch toward lipid oxidation with marked UCP3 up-regulation not associated with increased basal, fatty acid- or ROS-induced uncoupling or enhanced GDP effects. Biochim Biophys Acta, 2010. 1797(6-7): p. 968-80.

27. Tahara, E.B., F.D. Navarete, and A.J. Kowaltowski, Tissue-, substrate-, and sitespecific characteristics of mitochondrial reactive oxygen species generation. Free Radic Biol Med, 2009. 46(9): p. 1283-97.

28. Mansouri, A., et al., Alterations in mitochondrial function, hydrogen peroxide release and oxidative damage in mouse hind-limb skeletal muscle during aging. Mech Ageing Dev, 2006. 127(3): p. 298-306.

29. Hoehn, K.L., et al., Insulin resistance is a cellular antioxidant defense mechanism. Proc Natl Acad Sci U S A, 2009. 106(42): p. 17787-92.

30. Yuzefovych, L., G. Wilson, and L. Rachek, Different effects of oleate vs. palmitate on mitochondrial function, apoptosis, and insulin signaling in L6 skeletal muscle cells: role of oxidative stress. Am J Physiol Endocrinol Metab, 2010. 299(6): p. E1096-105.

31. Hansen, L.L., et al., Insulin signaling is inhibited by micromolar concentrations of $\mathrm{H}(2) \mathrm{O}(2)$. Evidence for a role of $\mathrm{H}(2) \mathrm{O}(2)$ in tumor necrosis factor alpha-mediated insulin resistance. J Biol Chem, 1999. 274(35): p. 25078-84.

32. Bonnard, C., et al., Mitochondrial dysfunction results from oxidative stress in the skeletal muscle of diet-induced insulin-resistant mice. J Clin Invest, 2008. 118(2): p. 789-800.

33. Ruiz-Ramirez, A., et al., High-sucrose diet increases ROS generation, FFA accumulation, UCP2 level, and proton leak in liver mitochondria. Am J Physiol Endocrinol Metab, 2011. 301(6): p. E1198-207.

34. Goldstein, B.J., K. Mahadev, and X. Wu, Redox paradox: insulin action is facilitated by insulin-stimulated reactive oxygen species with multiple potential signaling targets. Diabetes, 2005. 54(2): p. 311-21.

35. Rhee, S.G., Cell signaling. H2O2, a necessary evil for cell signaling. Science, 2006. 312(5782): p. 1882-3.

36. Loh, K., et al., Reactive oxygen species enhance insulin sensitivity. Cell Metab, 2009. 10(4): p. 260-72. 
37. McClung, J.P., et al., Development of insulin resistance and obesity in mice overexpressing cellular glutathione peroxidase. Proc Natl Acad Sci U S A, 2004. 101(24): p. 8852-7.

38. Yokota, T., et al., Oxidative stress in skeletal muscle impairs mitochondrial respiration and limits exercise capacity in type 2 diabetic mice. Am J Physiol Heart Circ Physiol, 2009. 297(3): p. H1069-77.

39. Abdul-Ghani, M.A., et al., Mitochondrial reactive oxygen species generation in obese non-diabetic and type 2 diabetic participants. Diabetologia, 2009. 52(4): p. 574-82.

40. Hoehn, K.L., et al., IRS1-independent defects define major nodes of insulin resistance. Cell Metab, 2008. 7(5): p. 421-33.

41. Turner, N., et al., Excess lipid availability increases mitochondrial fatty acid oxidative capacity in muscle: evidence against a role for reduced fatty acid oxidation in lipidinduced insulin resistance in rodents. Diabetes, 2007. 56(8): p. 2085-92.

42. Garcia-Roves, P., et al., Raising plasma fatty acid concentration induces increased biogenesis of mitochondria in skeletal muscle. Proc Natl Acad Sci U S A, 2007. 104(25): p. 10709-13.

43. Ristow, M., et al., Antioxidants prevent health-promoting effects of physical exercise in humans. Proc Natl Acad Sci U S A, 2009. 106(21): p. 8665-70.

44. Strobel, N.A., et al., Antioxidant supplementation reduces skeletal muscle mitochondrial biogenesis. Med Sci Sports Exerc, 2011. 43(6): p. 1017-24.

45. Higashida, K., et al., Normal adaptations to exercise despite protection against oxidative stress. Am J Physiol Endocrinol Metab, 2011. 301(5): p. E779-84.

46. Mogensen, M., et al., Mitochondrial respiration is decreased in skeletal muscle of patients with type 2 diabetes. Diabetes, 2007. 56(6): p. 1592-9.

47. Barazzoni, R., et al., Fatty acids acutely enhance insulin-induced oxidative stress and cause insulin resistance by increasing mitochondrial reactive oxygen species (ROS) generation and nuclear factor-kappaB inhibitor (IkappaB)-nuclear factor-kappaB (NFkappaB) activation in rat muscle, in the absence of mitochondrial dysfunction. Diabetologia, 2011.

48. Anisimov, V.N., et al., Effects of the mitochondria-targeted antioxidant SkQ1 on lifespan of rodents. Aging (Albany NY), 2011. 3(11): p. 1110-9. 


\section{CHAPTER 3}

\section{A two-week treatment with 2,4-dinitrophenol does not affect muscle substrate oxidation and glucose homeostasis in WT and $\mathrm{db} / \mathrm{db}$ mice}




\section{ABSTRACT}

Induction of mitochondrial uncoupling has been shown to increase the metabolic rate as well as markers for mitochondrial proliferation in muscle and may thus prevent diet-induced obesity and insulin resistance. Here, we tested if mitochondrial uncoupling via administration of the chemical uncoupler 2,4-dinitrophenol (DNP) could improve muscle substrate metabolism and whole body glucose homeostasis. Wildtype (WT) and $\mathrm{db} / \mathrm{db}$ mice were subjected to daily DNP administrations for the duration of 2 weeks. In vivo glucose homeostasis was determined by performing i.p. glucose tolerance tests while muscle substrate metabolism was measured ex vivo by ${ }^{14} \mathrm{C}$-palmitate and ${ }^{14} \mathrm{C}$-glucose oxidation assays in muscle homogenates. Muscle oxidative capacity was further evaluated by measuring markers for mitochondrial density and -biogenesis with western blot and RT-PCR. Muscle fat content was quantified by Oil Red O stainings. We found no effects of DNP on muscle glucose and palmitate oxidation, intramuscular lipid accumulation or glucose tolerance independent of the genotype. In addition, we did not detect differences in markers for mitochondrial density upon DNP treatment. Our results argue against potential beneficial effects of DNP-treatment on muscle substrate oxidation and insulin sensitivity in (diabetic) mice. 


\section{INTRODUCTION}

In skeletal muscle, accounting for $\sim 80 \%$ of insulin-stimulated glucose uptake, insulin sensitivity is negatively correlated to the amount of (ectopic) fat within muscle cells [1-3] resulting from an imbalance between fatty acid supply and oxidation. With respect to the latter, type 2 diabetes mellitus (T2DM) patients and insulin-resistant subjects are indeed characterized by disturbances in skeletal muscle mitochondrial oxidative capacity [4-6]. Importantly, we and others have shown that interventions, such as: exercise training, calorie restriction, and resveratrol supplementation, which positively affect muscle (fat) oxidation also result in improvements in skeletal muscle insulin sensitivity and metabolic health, both in obese subjects and T2DM patients [7-10].

Mitochondrial uncoupling is the process in which the mitochondrial proton gradient is dissociated from ATP production, which results in the dissipation of energy as heat. To compensate for the loss of proton gradient due to proton leak an excess of substrates will need to be oxidized in order to maintain an adequate ATP synthesis rate. By influencing the mitochondrial proton gradient in skeletal muscle, the metabolic rate and also insulin action can be significantly enhanced [11-15]. Mitochondrial uncoupling can be facilitated by uncoupling proteins (UCPS) although in mammals UCPs are demonstrated to be tissue selective and poorly active in skeletal muscle, as indicated by the low prevalence of obesity in knockout animal models [16-19]. On the other hand, mitochondrial uncoupling induced by ectopic expression of UCP1 [13, 20] as well as overexpression of UCP3 [21] in skeletal muscle has been shown to increase whole body energy expenditure and decrease adiposity. Alternatively, induction of uncoupling by chemical compounds such as 2,4-dinitrophenol (DNP) has been shown to be efficient and physiologically feasible. In vivo studies have shown that in low doses, DNP could have beneficial effects on lifespan and metabolic health in Saccharomyces cerevisiae [22], Drosophila melanogaster [23] and rodents [24, 25]. More specifically, chronic DNP exposure has been shown effective in decreasing body weight, fasting blood glucose, insulin and triglyceride levels in mice [24]. These changes were most likely a result of decreased efficiency of ATP production and increased catabolic fluxes since increased oxygen consumption rates were observed in tissues such as brain, liver and heart [24]. In rats, oral administration of DNP instantly resulted into an improved oxidation capacity of both fatty acids and glucose in skeletal muscle [25]. Furthermore, mice receiving DNP via an intraperitoneal injection [26] or drinking water [27] showed an elevated expression of genes involved in mitochondrial biogenesis, such as PGC1 $\alpha$ and NRF-1, in skeletal muscle, liver, and adipose tissue. Mitochondrial biogenesis may compensate for the decreased efficiency in ATP production by enhancing the number of functional mitochondria. Together, 
these data imply a beneficial effect of mitochondrial uncoupling on the aetiology of metabolic diseases in which muscle oxidative capacity is affected.

Since type 2 diabetes and insulin resistance are associated with a reduced mitochondrial capacity in skeletal muscle, we hypothesized that mitochondrial uncoupling could be beneficial in the treatment of type 2 diabetes mellitus, by augmenting the capacity for substrate oxidation in this tissue. To this end, we used a diabetic mouse strain $(\mathrm{db} / \mathrm{db})$ and tested the effects of a 2-week oral administration of DNP on muscle substrate oxidation, IMCL accumulation and glucose homeostasis in healthy vs. insulin resistant mice.

\section{MATERIALS AND METHODS}

\section{Animals}

Twelve-week old male C57BI/6 mice (WT) and B6.BKS(D)-Leprdb/J (db/db) were purchased from Charles River (Maastricht, The Netherlands) and housed individually in a controlled environment $\left(21^{\circ} \mathrm{C}\right)$ with a $12 \mathrm{~h}$ light: $12 \mathrm{~h}$ dark cycle. Standard chow (Ssniff, Soest, Germany) and tap water were available ad libitum. All protocols were approved and conducted in accordance with Maastricht University animal Ethics Committee guidelines and complied with the principles of laboratory animal care.

\section{Chemicals}

All chemicals were purchased from Sigma Aldrich (St. Louis, MO, USA) unless indicated otherwise.

\section{Dose optimization}

Dosing experiments were performed to test the optimal and maximum tolerated dose of DNP for WT and $\mathrm{db} / \mathrm{db}$ mice. Thus, mice $(n=3)$ received a daily oral gavage with either $1,5,10$, 20,30 or $45\left(L_{50}\right) \mathrm{mg} / \mathrm{kg}$ DNP for the duration of 2-weeks while monitoring the survival rate, body weight and food consumption.

\section{Long-term DNP treatment}

After establishing the optimal dose, WT and $\mathrm{db} / \mathrm{db}$ mice ( $\mathrm{n}=8$ per group) received a daily (at the end of the light cycle; at 18:00h) dose of either DNP (experimental groups, $20 \mathrm{mg} / \mathrm{kg}$ and $30 \mathrm{mg} / \mathrm{kg}$ for $\mathrm{db} / \mathrm{db}$ and WT mice respectively) or saline (control groups) by oral gavage for a total period of 2 weeks. Body weight and food consumption were monitored every 2 days. On day 12 of the intervention period, mice underwent an intraperitoneal (i.p.) glucose tolerance test. At the end of the study, mice received a final dose of DNP or saline at 18:00h and were sacrificed the next morning to avoid studying the acute effects of the final dose. On the last 
experimental day, all mice were fasted for 6 hours, sedated by a mixture of $79 \%(\mathrm{vol} / \mathrm{vol})$ of $\mathrm{CO}_{2}$ and $21 \%$ (vol/vol) of $\mathrm{O}_{2}$, and killed by cervical dislocation. Subsequently, gastrocnemius muscles were rapidly removed and directly used for ex vivo substrate oxidation assays. In addition, tibialis anterior muscles were excised, frozen in liquid nitrogen-cooled isopentane (2-methyl-butane, Fluka, Zwijndrecht, the Netherlands) and stored at $-80^{\circ} \mathrm{C}$ until further analysis.

\section{Acute DNP exposure}

In order to determine the acute effects of DNP treatment, separate groups of WT mice $(n=5)$ received a single dose $(30 \mathrm{mg} / \mathrm{kg})$ of DNP vs. saline in the fasted state. After 5 hours, mice were sacrificed and hind limb muscles were rapidly excised. The gastrocnemius muscle was used directly for ex vivo substrate oxidation assays. Tibialis anterior muscles were excised and stored at $-80^{\circ} \mathrm{C}$ for gene expression analyses.

\section{Glucose tolerance tests and plasma measurements}

Following a 6 -hour fast, mice received a bolus of glucose $(2 \mathrm{mg} / \mathrm{g}$ body weight, $200 \mathrm{~mL}$ of sterile solution) intraperitoneally. Blood samples were taken before $(t=0)$ and $15,30,60$, and 120 minutes after the glucose injection by bleeding the tail vein. Blood samples were collected in microtiter tubes (BD, Franklin Lakes, NJ, USA), and plasma was separated by centrifugation at $15,000 \mathrm{~g}$ for 10 minutes and stored at $-20^{\circ} \mathrm{C}$. Blood glucose was measured using a spectrophotometric assay with glucose HK CP reagents (Horiba ABX Diagnostics). A standard curve was made of D-(+)-glucose (ICN Biomedicals, Inc) and precinorm U (Roche Diagnostics $\mathrm{GmbH}$ ) was used as an internal control. Incremental areas under the curve were calculated from the blood glucose levels during the glucose tolerance test.

\section{Ex vivo substrate oxidation metabolism}

Ex vivo fatty acid (FA) and glucose oxidation were assessed in whole muscle (gastrocnemius) homogenates as previously described [28]. Briefly, gastrocnemius muscles were homogenized in $1.5 \mathrm{ml}$ SET buffer (Sucrose $250 \mathrm{mM}$, Tris-HCl 10mM, ATP 2mM, EDTA $1 \mathrm{mM})$. Homogenates were plated in a modified 48-well trapping device [29]. Reactions were started by adding a combination of cold and hot substrates (for FA oxdition: palmitate $0.2 \mathrm{mM}$, $\left[1-{ }^{14} \mathrm{C}\right]$-palmitate $(1 \mu \mathrm{Ci} / \mathrm{ml}$, Perkin-Elmer, Boston, USA) $0.02 \mathrm{mM}$; for glucose oxidation: glucose $10 \mathrm{mM}$, [U- $\left.{ }^{14} \mathrm{C}\right]$-labeled glucose $(1 \mu \mathrm{Ci} / \mathrm{ml}$, Perkin-Elmer, Boston, USA $\left.)\right)$ and reaction media (sucrose $125 \mathrm{mM}, \mathrm{KH}_{2} \mathrm{PO}_{4} 25 \mathrm{mM}, \mathrm{KCL} 200 \mathrm{mM}$, L-carnitine $2.5 \mathrm{mM}$, malic acid $0.25 \mathrm{mM}$, Tris- $\mathrm{HCl} 20 \mathrm{mM}$, DTT 2.5mM, NAD ${ }^{+} 0.25 \mathrm{mM}$, ATP $4 \mathrm{mM}$, Coenzyme A $0.125 \mathrm{mM}$ ) to each well. Substrates were coupled to FA-free BSA. The trapping device was sealed and incubated for 2 hours at $37^{\circ} \mathrm{C}$. After incubation, reactions were stopped by adding $70 \%$ 
perchloric acid and incubating the trapping device for 1 hour at room temperature. The produced ${ }^{14} \mathrm{CO}_{2}$ was trapped in the adjacent well containing $1 \mathrm{M} \mathrm{NaOH} .{ }^{14} \mathrm{CO}_{2}$ was quantified by liquid scintillation counting. Data were corrected for protein content. Protein content in muscle homogenates was determined with a DC kit (BioRad) according to manufacturer's instructions. Data were normalized to WT saline-treated animals.

\section{Intramyocellular lipid (IMCL) content}

To quantify the neutral lipid storage in skeletal muscle, cryosections $(5 \mu \mathrm{m})$ of tibialis anterior muscles were stained with Oil Red O (ORO) as previously described [30]. ORO staining was combined with laminin staining (L-9393. Sigma, St Louis, USA) to visualize the cell membranes. Sections were examined using a Nikon E800 fluorescence microscope (Uvikon, Bunnik, The Netherlands). Digital images were captured and processed using Lucia G/F 5.49 image analysis software (Nikon, Dusseldorf, Germany). IMCL content was calculated as per cell surface area. The lipid storage in muscle was normalized to the mean IMCL content in tibialis anterior muscle of saline-treated WT mice.

\section{Western Blot}

Tibialis anterior muscles were homogenized in lysis buffer (10\% NP40, 10\% SDS, 100mM PMSF in PBS) supplemented with a phosphatase and protease inhibitor cocktail (Roche). Standard SDS-PAGE, blocking and incubation protocols were followed. Blots were incubated overnight at room temperature with primary antibodies against PGC1 $\alpha$ (516557, Calbiochem, Darmstadt, Germany) or OXPHOS proteins (MS601; MitoSciences, Eugene, OR, USA). After the primary incubation period, appropriate secondary antibodies (Invitrogen, Paisley, UK) were applied and blots were visualized using the Odyssey Near Infrared Imager (Licor Biosciences, Westburg, Leusden, The Netherlands). Blots were quantified using Quantity One software (version 4.6.9; Biorad) and protein levels were expressed as arbitrary units $(A U)$.

\section{Enzyme activities}

Citrate synthase (CS) activity was measured in tibialis anterior muscle homogenates as previously described [31, 32]. In short, muscle homogenates were sonicated and centrifuged at $13,000 \mathrm{~g}$ for 10 minutes. Supernatants were used for the CS activity assay. Per well of a 96 wells plate $5 \mu \mathrm{l}$ sample $(1 \mathrm{mg} / \mathrm{ml}$ ), $250 \mu$ l reagent A (Tris $100 \mathrm{mM}$, DTNB $100 \mu \mathrm{M}$, Acetyl CoA $50 \mu \mathrm{M}$ ), and $5 \mu$ l start reagent (oxaloacetic acid $25 \mathrm{mM}$ ) were added. Absorption was measured at $412 \mathrm{~nm}$ every 21 seconds during 20 minutes at $37^{\circ} \mathrm{C}$ 


\section{Gene expression}

To explore whether acute DNP exposure affects the expression of genes involved in mitochondrial biogenesis, expression of the following genes was assessed by RT-PCR: PGC1 $\alpha$, PGC1 $\beta$, NRF1, and TFAM. Briefly, total RNA was isolated from tibialis anterior muscle samples using the acid phenol method of Chomczynski and Sacchi [33]. Subsequently, cDNA was made using the High Capacity RNA-to-cDNA Kit (Applied Biosystems, Branchburg, NJ, USA). Gene expression was quantified by Real-time PCR (ABI 7900 sequence detector, Applied Biosystems, Branchburg, NJ, USA) using the following program; one cycle at $50^{\circ} \mathrm{C}$ for $2 \mathrm{~min}$, then $95^{\circ} \mathrm{C}$ for $10 \mathrm{~min}$ followed by 40 cycles of $95^{\circ} \mathrm{C}$ for $15 \mathrm{sec}$ and $60^{\circ} \mathrm{C}$ for $1 \mathrm{~min}$. The absolute quantification of each gene was determined by a standard curve and was normalized to the expression of the housekeeping gene B2M.

\section{Statistics}

Results are expressed as means \pm SEM. Two-way ANOVA analyses were performed to analyze effects of genotype (WT vs. db/db), intervention (saline vs. DNP) and interaction (genotype * intervention). For the acute studies, unpaired t-tests were applied. Significance was set at $p<0.05$. All statistical analyses were performed using GraphPad Prism 5.0a Macintosh Version.

\section{RESULTS}

\section{Dose testing}

As a proof of principle, we first tested the capacity of DNP to induce mitochondrial uncoupling in isolated skeletal muscle mitochondria. DNP clearly increased mitochondrial respiration not coupled to ATP synthesis, in a dose-dependent manner (figure 1).

Subsequently, we performed dosing experiments to obtain a maximum tolerated in vivo dose for the 2-week intervention period. WT mice tolerated all doses $\leq 30 \mathrm{mg} / \mathrm{kg}$, i.e. all WT mice survived the 2-week intervention period with no effects of DNP treatment on body weight and food intake (data not shown). However, none of the WT mice $(n=3)$ survived the $45 \mathrm{mg} / \mathrm{kg}$ dose of DNP (figure 2A).

Analogously, all $\mathrm{db} / \mathrm{db}$ mice tolerated DNP doses $\leq 10 \mathrm{mg} / \mathrm{kg}$. Also in the $\mathrm{db} / \mathrm{db}$ mice no effects on body weight and food intake upon DNP were observed (data not shown). In contrast to the WT mice however, none of the $\mathrm{db} / \mathrm{db}$ mice survived the 2-week intervention period upon the $30 \mathrm{mg} / \mathrm{kg}$ dose (figure $2 \mathrm{~B}$ ), whereas $83 \%$ of the treated $\mathrm{db} / \mathrm{db}$ mice survived the 2-week intervention period upon $20 \mathrm{mg} / \mathrm{kg}$ (figure 2B). Based on these outcomes, we established $30 \mathrm{mg} / \mathrm{kg}$ and $20 \mathrm{mg} / \mathrm{kg}$ of DNP as optimal and maximally tolerated doses for WT mice and $\mathrm{db} / \mathrm{db}$ mice, respectively. 


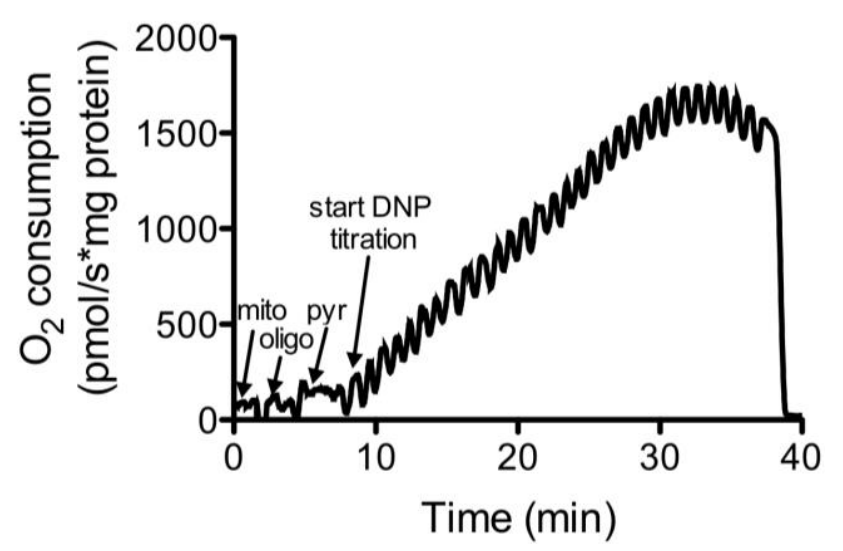

Figure 1. Representative experiment of mitochondrial oxygen consumption (pmol/s*mg) not coupled to ATP synthesis upon increasing DNP concentrations. Mitochondria $(0.1 \mathrm{mg} / \mathrm{ml})$ were isolated from rat tibialis anterior muscles and used for respiration measurements at $37^{\circ} \mathrm{C}$ using a two-chambered Oxygraph (Oroboros Instruments, Innsbruck, Austria). Mitochondria were incubated in medium consisting of $100 \mathrm{mM}$ sucrose, $50 \mathrm{mM}$ $\mathrm{KCl}, 20 \mathrm{mM} \mathrm{K}^{+}$-TES ( $\mathrm{pH} 7.2$ ), $2 \mathrm{mM} \mathrm{MgCl}_{2}, 1 \mathrm{mM}$ EDTA, $4 \mathrm{mM} \mathrm{KH}_{2} \mathrm{PO}_{4}, 3 \mathrm{mM}$ malate and $0.1 \%$ (w/v) bovine serum albumin (BSA). ATP-synthase was blocked by adding oligomycin $(1 \mu \mathrm{g} / \mathrm{ml})$ to the mitochondria. Pyruvate $(5 \mathrm{mM})$ was added as a substrate. Uncoupled mitochondrial respiration (state $U$ respiration) was quantified by titrating DNP (10mM), $2.5 \mu \mathrm{M}$ per titration step.
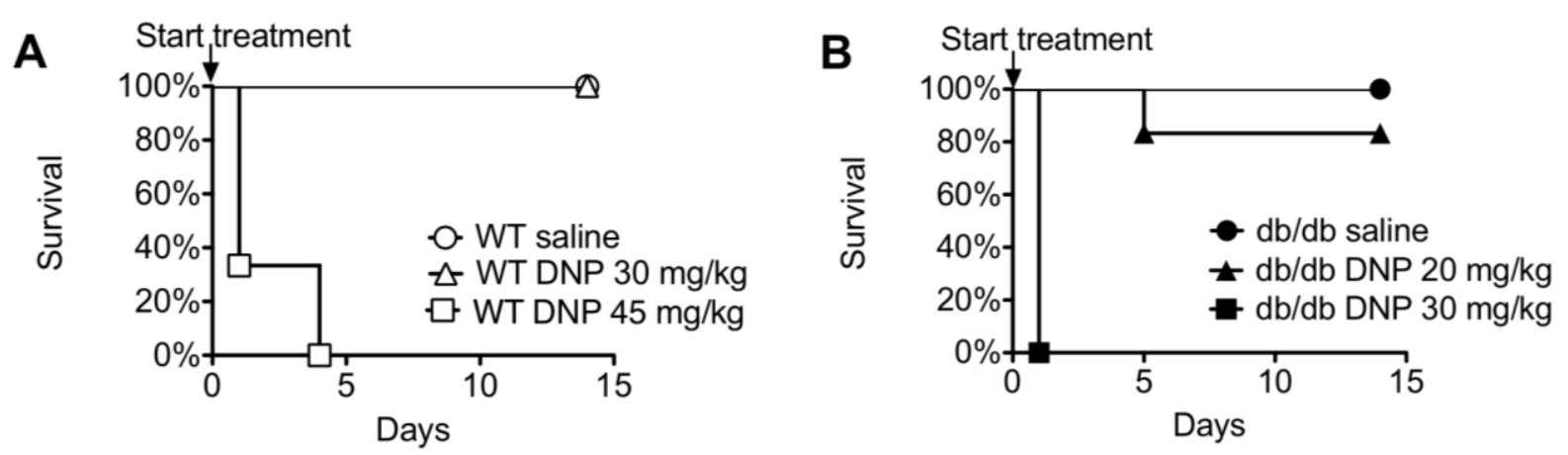

Figure 2. Survival curves for WT mice upon 0 (saline), 30 or $45 \mathrm{mg} / \mathrm{kg} \mathrm{DNP}$ ( $\mathrm{n}=3 \mathrm{per}$ dose) (A); and db/db mice dosed with 0 (saline), 20 or $30 \mathrm{mg} / \mathrm{kg}$ DNP ( $\mathrm{n}=3$ per dose) (B). All mice tolerated DNP doses $\leq 10 \mathrm{mg} / \mathrm{kg}$. WT mice survived upon DNP doses below the $30 \mathrm{mg} / \mathrm{kg}$ whereas $\mathrm{db} / \mathrm{db}$ mice tolerated all DNP doses $<20 \mathrm{mg} / \mathrm{kg}$ (data not shown).

\section{Body weight}

Body weights after 2 weeks of DNP/saline administration were similar in the WT groups $(24.2 \pm 0.9 \mathrm{~g}$ vs. $24.7 \pm 1.1 \mathrm{~g}$ after saline vs. $D N P$, respectively; $\mathrm{p}=0.34)$ as well as in the $\mathrm{db} / \mathrm{db}$ mice groups $(40.8 \pm 5.5 \mathrm{~g}$ vs. $41.8 \pm 1.3 \mathrm{~g}$ after saline vs. $D N P$, respectively; $p=0.65)$. Thus, apart from the anticipated genotype effect in body weight $(p<0.0001)$, there was no treatment effect $(p=0.48)$ (figure $3 A)$. Although $\mathrm{db} / \mathrm{db}$ mice $(6.9 \pm 0.5 \mathrm{~g} /$ day vs. $7.0 \pm 0.4 \mathrm{~g} /$ day in saline vs. DNP treated db/db mice) ate significantly $(p=0.0004)$ more than WT mice $(4.8 \pm 0.2 \mathrm{~g} /$ day 
vs. $5.7 \pm 0.3 \mathrm{~g} /$ day in saline vs. DNP treated WT mice), we did not detect any differences in food consumption upon DNP treatment $(p=0.15)$ (figure 3B), neither in WT nor in $\mathrm{db} / \mathrm{db}$ mice.

A

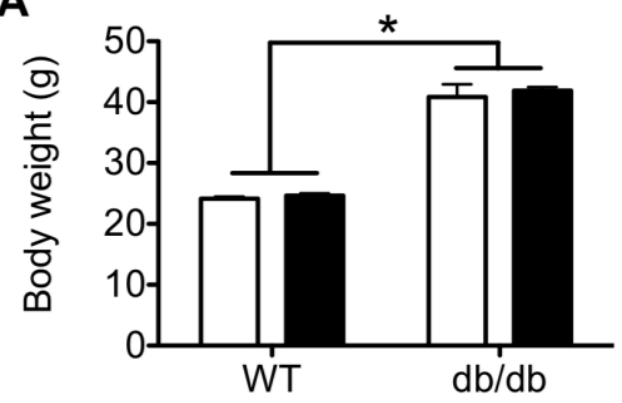

B

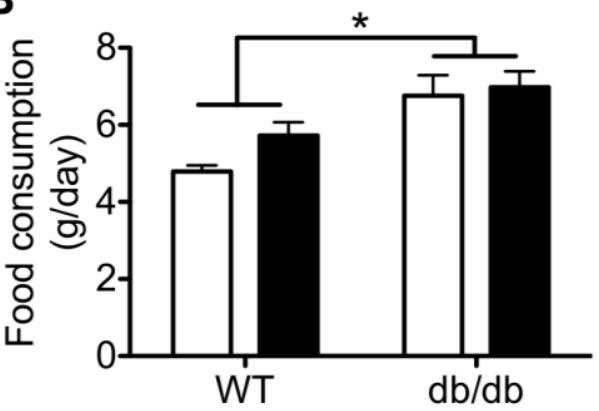

Figure 3. Body weight (A), and Food consumption (B) of mice ( $n=8$ per group) at day 14 of the intervention period. Open bars represent control mice (saline-treated) and black bars symbolize DNP-treated mice. *: genotype difference, $p<0.05$.

\section{Glucose tolerance}

Next, we tested whether DNP treatment was associated with changes in whole body glucose tolerance by performing i.p. glucose tolerance tests in WT and db/db mice on day 12 of the intervention period. An apparent difference in fasting blood glucose levels $(13.7 \pm 0.4$ vs. $40.5 \pm 2.4 \mathrm{mM}, \mathrm{p}<0.0001$ ) and glucose tolerance (AUC 2314.0 \pm 54.7 vs. 8186.6 \pm 495.3 , $\mathrm{p}<0.0001$, figure 4) between WT and $\mathrm{db} / \mathrm{db}$ mice was found. Moreover, we detected $\mathrm{a}$ significant interaction $(p=0.0033)$ between genotype and intervention on fasting blood glucose levels, i.e. fasting glucose levels were lower in DNP-treated $\mathrm{db} / \mathrm{db}$ mice vs. salinetreated animals $(p=0.005)$ whereas DNP had no effect on this parameter in WT animals. However, glucose response after i.p. glucose injection was not improved by DNP, neither in WT nor in $\mathrm{db} / \mathrm{db}$ mice ( $\mathrm{p}=0.88$, figure 4 ) indicating that DNP did not influence glucose tolerance.
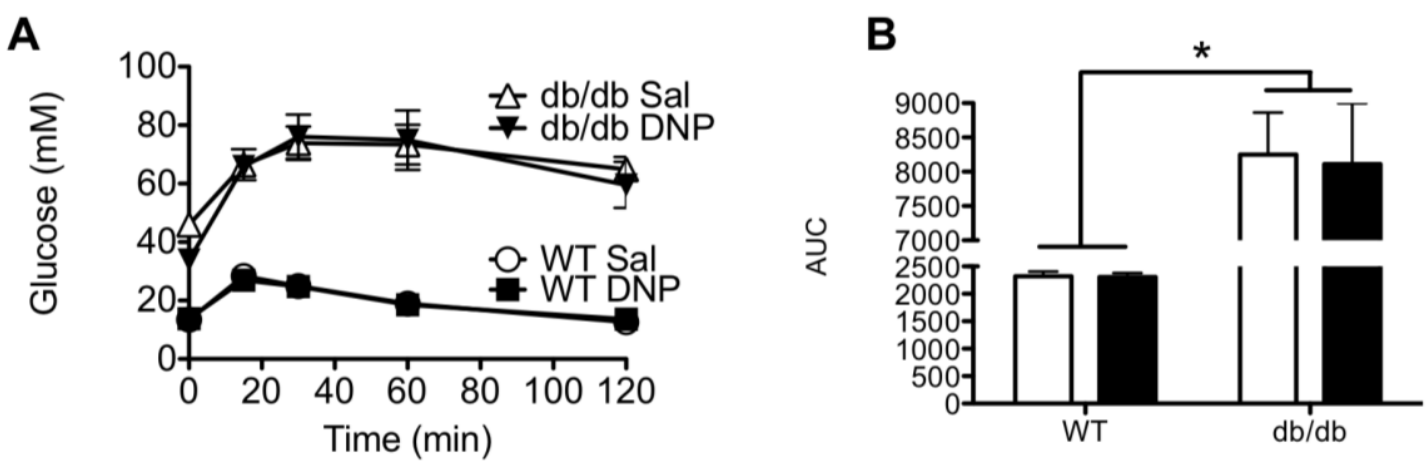

Figure 4. Intraperitoneal glucose tolerance test. Mice where injected with glucose ( $2 \mathrm{mg} / \mathrm{g}$ body weight) at $\mathrm{t}=0$. Plasma glucose levels over time (A). Area under the curve (AUC) (B). Open and closed bars represent salineand DNP-treated mice respectively. *: significant genotype difference, $p<0.05$. 


\section{Ex vivo substrate oxidation}

To test if DNP treatment is able to increase the capacity of skeletal muscle to oxidize fatty acids and glucose, we performed ex vivo substrate oxidation assays in muscle from WT and $\mathrm{db} / \mathrm{db}$ mice. As depicted in figure $5 \mathrm{~A}$, complete palmitate oxidation to $\mathrm{CO}_{2}$ tended to be reduced in $\mathrm{db} / \mathrm{db}$ mice $(1.07 \pm 0.14$ vs. $0.72 \pm 0.09 \mathrm{nmol} / 2 \mathrm{hr} / \mathrm{mg}$ protein for WT and $\mathrm{db} / \mathrm{db}$ mice respectively, $p=0.07$ ) whereas complete glucose oxidation was significantly lowered in $\mathrm{db} / \mathrm{db}$ mice $(1.04 \pm 0.13$ vs. $0.50 \pm 0.07 \mathrm{nmol} / 2 \mathrm{hr} / \mathrm{mg}$ protein for WT and $\mathrm{db} / \mathrm{db}$ mice respectively, $\mathrm{p}=0.004$, figure $5 \mathrm{~B}$ ), as compared with $\mathrm{WT}$ mice. These results suggest that skeletal muscle from $\mathrm{db} / \mathrm{db}$ mice have a decreased capacity to oxidize substrates. DNP treatment did however not affect ex vivo palmitate $(p=0.46)$ or glucose $(p=0.66)$ oxidation in skeletal muscle, neither in WT nor in $\mathrm{db} / \mathrm{db}$ mice.

A

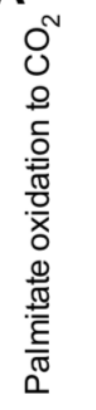

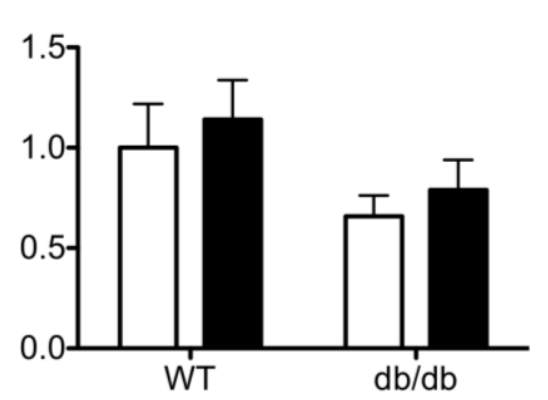

B

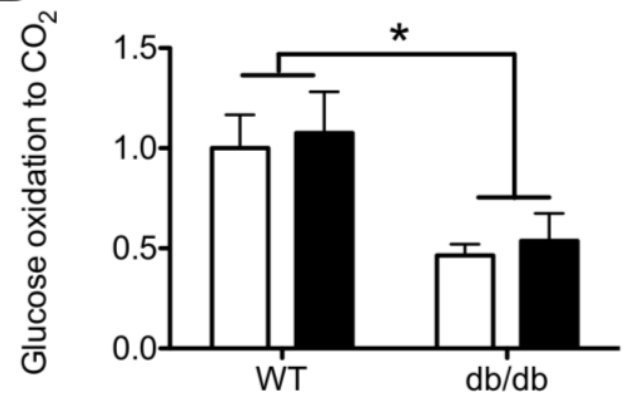

Figure 5. Ex vivo substrate metabolism assessed in whole muscle homogenates of the m. gastrocnemius. complete palmitate oxidation to $\mathrm{CO}_{2}(\mathbf{A})$, complete glucose oxidation to $\mathrm{CO}_{2}(\mathbf{B})$. Data are normalized to the saline-treated WT group. Open and closed bars represent saline- and DNP-treated mice respectively. * significant difference between genotypes, $\mathrm{p}<0.05$.

\section{Oil red $O$ staining}

In addition to the effects of DNP on glucose tolerance and muscle substrate oxidation, we also studied its effects on intramyocellular lipid (IMCL) accumulation by ORO staining. Quantification of IMCL levels revealed significantly higher levels in $\mathrm{db} / \mathrm{db}$ mice compared with WT mice ( $1.00 \pm 0.32$ vs. $2.12 \pm 0.67$ in saline- vs. DNP-treated WT mice; $12.43 \pm 4.33$ vs. $10.34 \pm 2.44$ in saline- vs. DNP-treated $\mathrm{db} / \mathrm{db}$ animals; $p=0.0003$, figure 6) but remained unaffected upon DNP treatment $(p=0.84)$. 
A

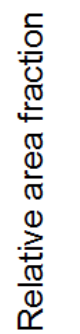

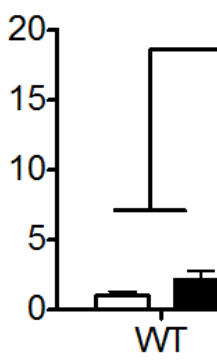

*

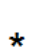

B
B

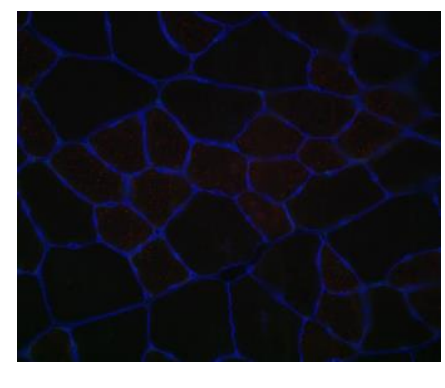

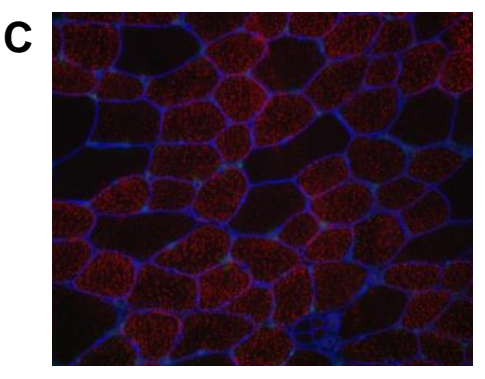

Figure 6. Quantification of ORO staining in tibialis anterior muscle of mice. IMCL levels are calculated as per cell surface area (A). The mean IMCL content in tibialis anterior muscle of WT mice was set to 1 . $\mathrm{N}=6-8$ mice per group. Open and closed bars stand for saline- and DNP-treated mice. Representative image of ORO in WT (B), and in $\mathrm{db} / \mathrm{db}$ mice $(\mathbf{C}){ }^{*}$ : significant genotype effect, $\mathrm{p}<0.05$.

\section{Parameters of mitochondrial density}

Protein levels of PGC1 $\alpha$ (figure 7A) and OXPHOS complexes (figure 7B) were similar between the two genotypes and also remained unchanged upon the DNP treatment.

In contrast, CS activity in skeletal muscle was significantly elevated in $\mathrm{db} / \mathrm{db}$ mice compared with WT mice $(P=0.006$, figure $7 C$ ). Unexpectedly, DNP treatment significantly decreased CS activity ( $P=0.032$, figure $7 C$ ). This decrease in CS activity appeared to be largely due to a decline in $\mathrm{db} / \mathrm{db}$ mice, although we did not observe a significant interaction between genotype and intervention $(p=0.17)$.

\section{Acute studies}

To explore the possibility that DNP administration has acute effects that diminish rapidly and therefore do not result in persistent metabolic changes, we also performed additional experiments in WT mice receiving a single dose of either saline $(n=5)$ or $30 \mathrm{mg} / \mathrm{kg}$ DNP $(n=5)$. Five hours after DNP dosage, skeletal muscle was harvested for palmitate (figure 8A) and glucose oxidation (figure $8 \mathrm{~B}$ ) assays. However, for both substrates, palmitate and glucose, complete oxidation was similar for both groups (palmitate: $1.00 \pm 0.10$ vs. $1.02 \pm 0.09$ $\mathrm{nmol} / 2 \mathrm{hr} / \mathrm{mg}$ protein in saline- vs. DNP-treated mice, $\mathrm{p}=0.90$; glucose: $1.00 \pm 0.14$ vs. $1.10 \pm 0.23 \mathrm{nmol} / 2 \mathrm{hr} / \mathrm{mg}$ protein in saline- vs. DNP-treated mice, $p=0.71)$. These data imply that also acute DNP administration does not affect substrate oxidation in skeletal muscle.

In addition we also assessed mRNA levels of PGC1 $\alpha$, PGC1 $\beta$, NRF1 and TFAM in skeletal muscle (figure 9), all genes involved in the regulation of mitochondrial biogenesis. In line with the oxidation results, we did not detect any effect of DNP on the transcription of these genes. If anything, PGC1 $\alpha$ gene expression tended to be decreased upon DNP $(p=0.09)$. 


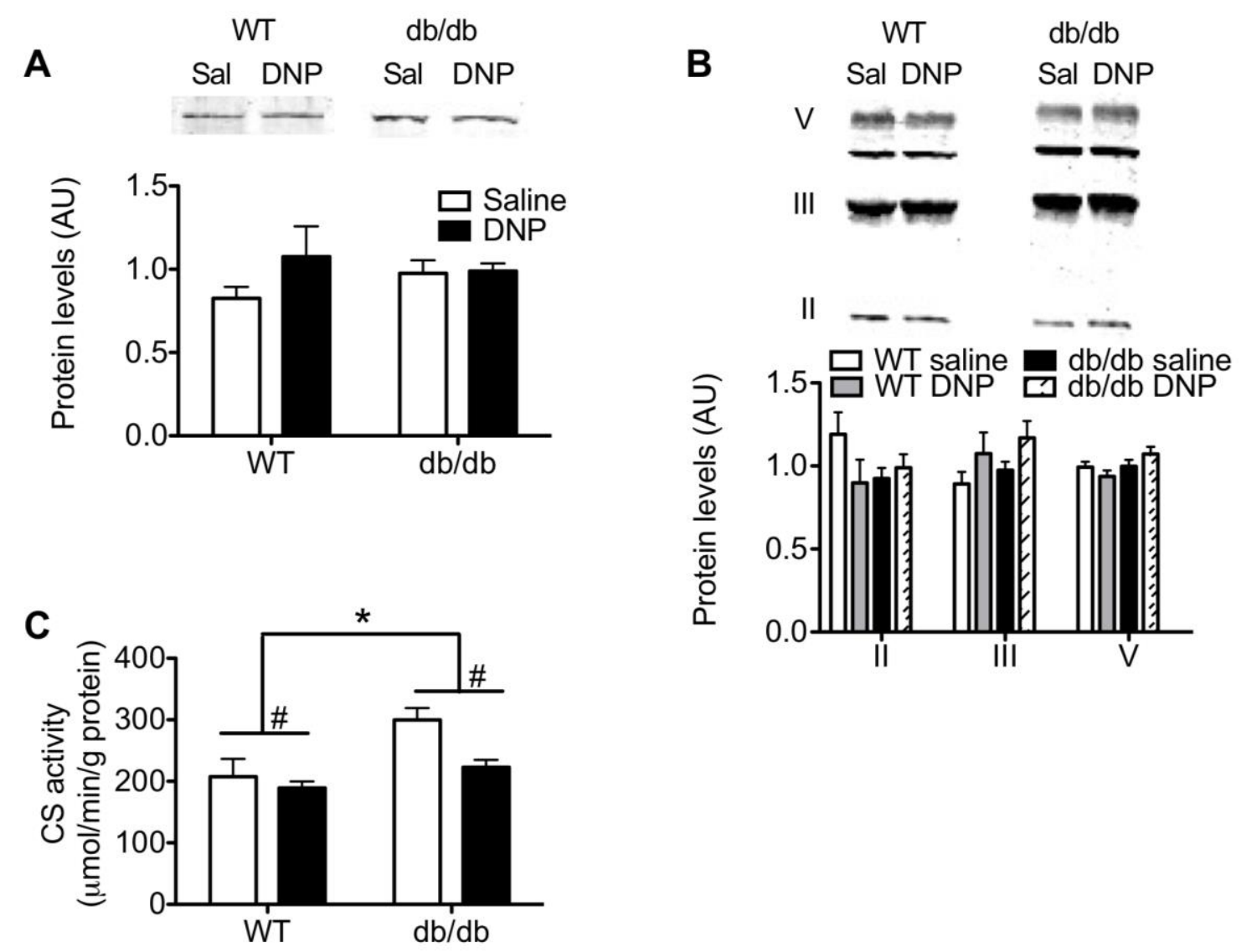

Figure 7. Quantification of western blots for PGC1 $\alpha(\mathbf{A})$, and OXPHOS complexes (B) determined in tibialis anterior muscle homogenates. Citrate synthase activity ( $\mu \mathrm{mol} / \mathrm{min} / \mathrm{g}$ protein) measured in whole muscle tissue homogenates $(\mathbf{C}){ }^{*}$ : significant genotype difference, $p<0.05$; \#: treatment effect, $p<0.05$.

A

O
0
0
0
.0
$\frac{0}{0}$
$\frac{0}{x}$
0
0
$\frac{0}{0}$
$\frac{\pi}{E}$
$\frac{1}{\pi}$
0

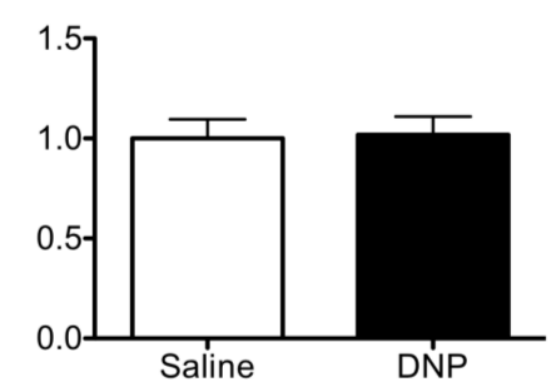

B

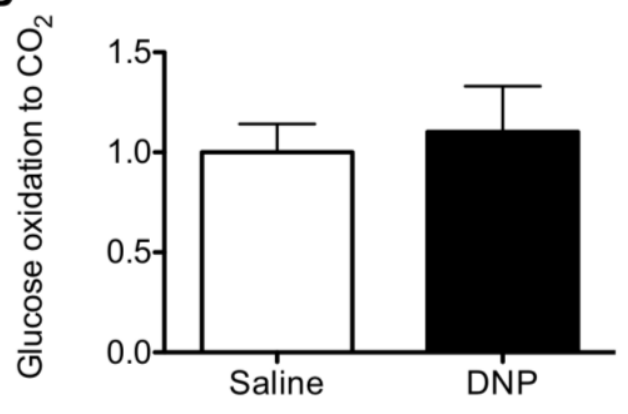

Figure 8. Ex vivo substrate oxidation was assessed in whole muscle homogenates of gastrocnemius from WT mice. Complete palmitate oxidation to $\mathrm{CO}_{2}(\mathbf{A})$, and complete glucose oxidation to $\mathrm{CO}_{2}(\mathbf{B})$. Data are normalized to saline-treated WT mice. 


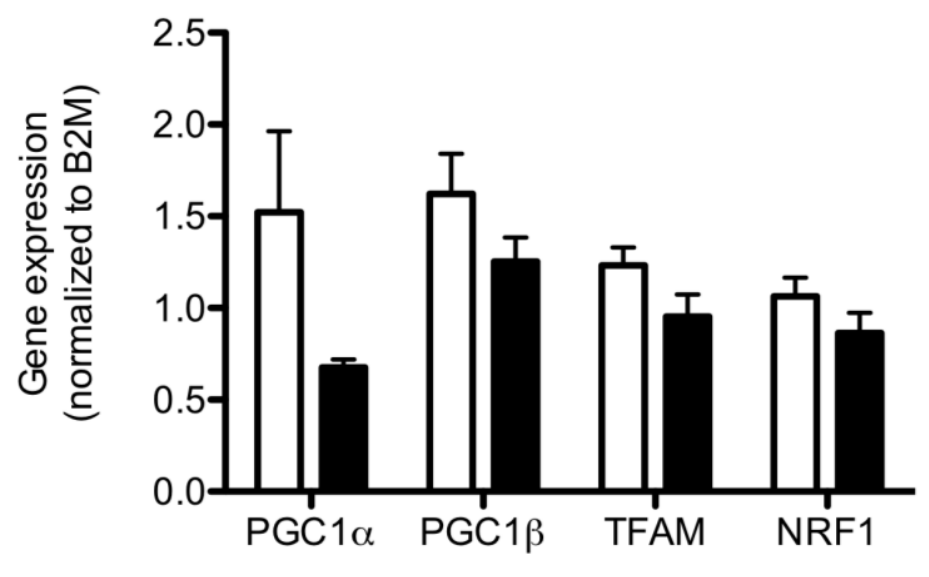

Figure 9. The acute effect of DNP on the mitochondrial biogenesis was determined in tibialis anterior muscles. Gene expression of PGC1 $\alpha$, PGC1 $\beta$, TFAM and NRF1 were normalized to the expression of the housekeeping gene B2M.

\section{DISCUSSION}

Type 2 diabetes mellitus (T2DM) and insulin resistance are associated with increased lipid accumulation and a reduced mitochondrial oxidative capacity in skeletal muscle. When food supply exceeds substrate oxidation, efficient metabolism causes continued energy storage resulting into obesity. Enhancing mitochondrial uncoupling, via overexpression of uncoupling protein 1 (UCP1), in skeletal muscle was shown to decrease metabolic efficiency, increase the metabolic rate and to protect against diet-induced obesity and insulin resistance [11]. In the present study we tested the hypothesis whether the induction of mitochondrial uncoupling via a 2-week treatment with the chemical uncoupler DNP could have beneficial effects on muscle metabolism and glucose homeostasis in healthy WT vs. diabetic $\mathrm{db} / \mathrm{db}$ mice. However, DNP did not affect muscle glucose and palmitate oxidation, intramuscular lipid accumulation or glucose tolerance. In addition, we did not detect differences in markers for mitochondrial density upon DNP treatment. These data argue against a potential beneficial role for DNP in promoting muscle substrate oxidation and insulin sensitivity.

Prior to the onset of the DNP intervention study, we performed dosing experiments to identify the maximum dose of DNP tolerated by WT and $\mathrm{db} / \mathrm{db}$ mice over a period of 2 weeks. We observed a lower tolerance of $\mathrm{db} / \mathrm{db}$ mice to DNP compared with WT, which could be explained by the difference in body composition between these mice. DNP accumulates more in tissues with higher lipid contents, and therefore, DNP can exert tissuespecific and genotype-specific effects upon systemic administration [24].

We found that daily administration of a substantial dose of DNP during a 2-week intervention period did not induce changes in body weight, neither in WT nor in $\mathrm{db} / \mathrm{db}$ mice. However, in a study in which (healthy) female Swiss mice were lifelong exposed to DNP $(0.06 \mathrm{mg} / \mathrm{kg})$ via drinking water, a $10 \%$ lower weight gain than controls was displayed [24]. In our study we applied an intervention period of 2 weeks. Hence, it could be that an intervention period of 2 weeks is not sufficient for DNP to induce changes in body weight in WT nor $\mathrm{db} / \mathrm{db}$ mice. Nevertheless, DNP has been reported extensively to cause rapid weight 
loss in humans when taken orally, in lower doses $(2-5 \mathrm{mg} / \mathrm{kg})$ as used in our study, for periods varying from a few days to a couple of weeks [34-36]. The cause of variability in sensitivity to DNP is still unclear, but possibly factors such as: species, body composition, genetic background, and hormonal or metabolic status are contributing to this inconsistency $[37,38]$.

Since mitochondrial uncoupling has been shown to increase glucose transport and insulin signaling in skeletal muscle [39, 40], we explored whether DNP exposure could affect glucose tolerance. Apart from the anticipated difference in glucose tolerance between WT and $\mathrm{db} / \mathrm{db}$ mice, and reduced fasting glucose levels in DNP-treated $\mathrm{db} / \mathrm{db}$ animals, DNP did not affect glucose tolerance, in neither genotype. These results were surprising, as mouse models in which muscle mitochondrial uncoupling was induced via overexpression of uncoupling proteins (UCPs), did demonstrated improved glucose homeostasis [11, 41, 42]. Transgenic ( $\mathrm{Tg}$ ) mice models overexpressing UCP1 (the first identified UCP) in skeletal muscle were protected form diet-induced obesity [11, 41]. Furthermore, UCP1 Tg animals had an enhanced glucose transport into the muscle upon a high fat diet when compared with WT, despite the presence of increased IMCL levels [11, 42]. In this context, more prolonged exposure to DNP may be required to induce improvements in glucose homeostasis. Of course, it remains possible that DNP has beneficial effects on (muscle) insulin sensitivity that does not become apparent in our whole-body glucose tolerance test. Whether DNP is capable to affect glucose homeostasis remains speculative, as currently, there are no other studies on the effects of DNP treatment on glucose tolerance under diabetic conditions.

To investigate whether DNP treatment affects the capacity of skeletal muscle to oxidize fatty acids and glucose in the current study, ex vivo substrate oxidation experiments were performed. Here, we found a reduced capacity for complete oxidation of glucose and palmitate in $\mathrm{db} / \mathrm{db}$ mice, supporting the notion that insulin resistance and T2DM is accompanied by a reduced muscle oxidative capacity [5, 6, 43, 44]. Upon DNP treatment, we anticipated that the capacity for substrate oxidation would be increased in order to maintain an adequate ATP synthesis rate. However, we were unable to detect an effect of DNP on glucose or palmitate oxidation in skeletal muscle, neither in WT nor in db/db mice. In line with these observations, we did not detect any differences between saline vs. DNP-treated animals with respect to markers for mitochondrial density or - biogenesis, i.e. PGC-1 $\alpha$ and OXPHOS protein levels were similar between saline and DNP-treated animals. If anything, we found a negative effect of DNP on mitochondrial density, as exemplified by CS activity, specifically in the $\mathrm{db} / \mathrm{db}$ mice. In this context, it was shown before that chronic exposure of tadpoles to DNP via their skin for 34 days resulted in higher cytochrome c oxidase activity per mitochondria but lower mitochondrial biogenesis, indicating that DNP-induced 
mitochondrial uncoupling is able to affect intrinsic mitochondrial function without influencing mitochondrial content [45].

Furthermore, we found elevated IMCL levels in $\mathrm{db} / \mathrm{db}$ mice as compared with WT mice, reflecting their insulin resistant phenotype. However, in line with the comparable muscle oxidative capacity, also IMCL levels in DNP-treated mice were similar to levels found in saline-treated animals, in both genotypes.

Due to the lack of changes in our 2-week intervention study we questioned whether DNP may have had acute and short-lasting effects that failed persist in order to affect metabolism, especially since all experiments were performed at least 12 hours after the last dose of DNP. Therefore, we also performed additional experiments in which we examined acute effects (after 5 hours) of one oral dose of DNP in WT mice. However, ex vivo substrate oxidation and the expression of several oxidative genes was similar between DNP-treated and control mice. In a comparable approach, Rohas et al (2007) treated WT and skeletal muscle-specific PGC1 $\alpha$ knockout mice with $50 \mathrm{mg} / \mathrm{kg}$ DNP via i.p. injection and found increased expression of PGC1 $\alpha, \mathrm{PGC} 1 \beta$, Cyt c, COXII, ATPase, and TFAM in WT mice, 5 hours after the injection [26]. However, these effects were completely absent in PGC1 $\alpha$ knockout animals, suggesting that $P G C 1 \alpha$ is required for the induction of mitochondrial electron transport genes induced by mild mitochondrial uncoupling [26]. In the present study, we were unable to confirm these findings of DNP on PGC1 $\alpha$ protein or gene expression neither on the short- nor on the long-term. However, it is important to note that we used a lower dose of DNP compared with Rohas et al (2007): $30 \mathrm{mg} / \mathrm{kg}$ instead of $50 \mathrm{mg} / \mathrm{kg}$, due to the high mortality rates. Besides dosage, also differences in administration (i.p. injection vs. oral gavage) or solvent (saline vs. DMSO) [46] may contribute to this discrepancy in results. When given ex vivo, we did however see that DNP was able to induce mitochondrial uncoupling, and increasing the dose of DNP in vivo resulted in premature death of the animals, often within a few days after the start of the intervention. Therefore, we are convinced that DNP was able to induce mitochondrial uncoupling but that the effects on skeletal muscle may be too mild to detect effects on metabolism within the timeframe of our study.

In conclusion, the aim of this study was to investigate whether DNP-induced mitochondrial uncoupling could have beneficial effects on muscle substrate oxidation and glucose homeostasis under diabetic conditions. Here we show however, that a 2-week daily administration of DNP in mice does not affect skeletal muscle substrate oxidation, IMCL levels nor glucose tolerance. Our data does not support a prominent role for chemical mitochondrial uncouplers in the improvement of metabolic health. Therefore, we could not validate DNP to be an appropriate therapy for T2DM. 


\section{Acknowledgements}

This study was supported by a $\mathrm{VICl}$ research grant for innovative research from the Netherlands Organization for Scientific Research (Grant 918.96.618).

\section{REFERENCES}

1. Petersen, K.F., et al., Impaired mitochondrial activity in the insulin-resistant offspring of patients with type 2 diabetes. N Engl J Med, 2004. 350(7): p. 664-71.

2. Savage, D.B., K.F. Petersen, and G.I. Shulman, Disordered lipid metabolism and the pathogenesis of insulin resistance. Physiol Rev, 2007. 87(2): p. 507-20.

3. Chow, L., A. From, and E. Seaquist, Skeletal muscle insulin resistance: the interplay of local lipid excess and mitochondrial dysfunction. Metabolism, 2010. 59(1): p. 7085.

4. Krssak, M., et al., Intramyocellular lipid concentrations are correlated with insulin sensitivity in humans: a 1H NMR spectroscopy study. Diabetologia, 1999. 42(1): p. 113-6.

5. Perseghin, G., et al., Intramyocellular triglyceride content is a determinant of in vivo insulin resistance in humans: a $1 \mathrm{H}-13 \mathrm{C}$ nuclear magnetic resonance spectroscopy assessment in offspring of type 2 diabetic parents. Diabetes, 1999. 48(8): p. 1600-6.

6. Pan, D.A., et al., Skeletal muscle triglyceride levels are inversely related to insulin action. Diabetes, 1997. 46(6): p. 983-8.

7. Hoeks, J., et al., Prolonged fasting identifies skeletal muscle mitochondrial dysfunction as consequence rather than cause of human insulin resistance. Diabetes, 2010. 59(9): p. 2117-25.

8. Jazet, I.M., et al., Loss of $50 \%$ of excess weight using a very low energy diet improves insulin-stimulated glucose disposal and skeletal muscle insulin signalling in obese insulin-treated type 2 diabetic patients. Diabetologia, 2008. 51(2): p. 309-19.

9. Phielix, E., et al., High oxidative capacity due to chronic exercise training attenuates lipid-induced insulin resistance. Diabetes, 2012. 61(10): p. 2472-8.

10. Timmers, S., et al., Calorie restriction-like effects of 30 days of resveratrol supplementation on energy metabolism and metabolic profile in obese humans. Cell Metab, 2011. 14(5): p. 612-22.

11. Li, B., et al., Skeletal muscle respiratory uncoupling prevents diet-induced obesity and insulin resistance in mice. Nat Med, 2000. 6(10): p. 1115-20.

12. Thrush, A.B., et al., Implications of mitochondrial uncoupling in skeletal muscle in the development and treatment of obesity. Febs J, 2013.

13. Adjeitey, C.N., et al., Mitochondrial uncoupling in skeletal muscle by UCP1 augments energy expenditure and glutathione content, while mitigating ROS production. Am J Physiol Endocrinol Metab, 2013. 305(3): p. E405-15.

14. Azzu, V., et al., The regulation and turnover of mitochondrial uncoupling proteins. Biochim Biophys Acta, 2010. 1797(6-7): p. 785-91.

15. Harper, J.A., K. Dickinson, and M.D. Brand, Mitochondrial uncoupling as a target for drug development for the treatment of obesity. Obes Rev, 2001. 2(4): p. 255-65.

16. Arsenijevic, D., et al., Disruption of the uncoupling protein-2 gene in mice reveals a role in immunity and reactive oxygen species production. Nat Genet, 2000. 26(4): p. 435-9.

17. Gong, D.W., et al., Lack of obesity and normal response to fasting and thyroid hormone in mice lacking uncoupling protein-3. J Biol Chem, 2000. 275(21): p. 162517.

18. Enerback, S., et al., Mice lacking mitochondrial uncoupling protein are cold-sensitive but not obese. Nature, 1997. 387(6628): p. 90-4.

19. Nabben, M., et al., Significance of uncoupling protein 3 in mitochondrial function upon mid- and long-term dietary high-fat exposure. FEBS Lett, 2011. 585(24): p. 4010-7. 
20. Couplan, E., et al., High level of uncoupling protein 1 expression in muscle of transgenic mice selectively affects muscles at rest and decreases their $\mathrm{Ilb}$ fiber content. J Biol Chem, 2002. 277(45): p. 43079-88.

21. Aguer, C., et al., Muscle uncoupling protein 3 overexpression mimics endurance training and reduces circulating biomarkers of incomplete beta-oxidation. FASEB J, 2013.

22. Barros, M.H., et al., Higher respiratory activity decreases mitochondrial reactive oxygen release and increases life span in Saccharomyces cerevisiae. J Biol Chem, 2004. 279(48): p. 49883-8.

23. Padalko, V.I., Uncoupler of oxidative phosphorylation prolongs the lifespan of Drosophila. Biochemistry (Mosc), 2005. 70(9): p. 986-9.

24. Caldeira da Silva, C.C., et al., Mild mitochondrial uncoupling in mice affects energy metabolism, redox balance and longevity. Aging Cell, 2008. 7(4): p. 552-60.

25. Hoehn, K.L., et al., Acute or chronic upregulation of mitochondrial fatty acid oxidation has no net effect on whole-body energy expenditure or adiposity. Cell Metab, 2010. 11(1): p. 70-6.

26. Rohas, L.M., et al., A fundamental system of cellular energy homeostasis regulated by PGC-1alpha. Proc Natl Acad Sci U S A, 2007. 104(19): p. 7933-8.

27. Cerqueira, F., F. Laurindo, and A. Kowaltowski, Mild mitochondrial uncoupling and calorie restriction increase fasting eNOS, akt and mitochondrial biogenesis. PloS one, 2011. 6(3): p. e18433.

28. Hulver, M.W., et al., Skeletal muscle lipid metabolism with obesity. Am J Physiol Endocrinol Metab, 2003. 284(4): p. E741-7.

29. Ukropcova, B., et al., Dynamic changes in fat oxidation in human primary myocytes mirror metabolic characteristics of the donor. J Clin Invest, 2005. 115(7): p. 1934-41.

30. Koopman, R., G. Schaart, and M.K. Hesselink, Optimisation of oil red O staining permits combination with immunofluorescence and automated quantification of lipids. Histochem Cell Biol, 2001. 116(1): p. 63-8.

31. Shepherd, D. and P.B. Garland, The kinetic properties of citrate synthase from rat liver mitochondria. Biochem J, 1969. 114(3): p. 597-610.

32. den Hoed, M., et al., Habitual physical activity in daily life correlates positively with markers for mitochondrial capacity. J Appl Physiol, 2008. 105(2): p. 561-8.

33. Chomczynski, P. and N. Sacchi, Single-step method of RNA isolation by acid guanidinium thiocyanate-phenol-chloroform extraction. Anal Biochem, 1987. 162(1): p. 156-9.

34. Colman, E., Dinitrophenol and obesity: an early twentieth-century regulatory dilemma. Regul Toxicol Pharmacol, 2007. 48(2): p. 115-7.

35. Grundlingh, J., et al., 2,4-dinitrophenol (DNP): a weight loss agent with significant acute toxicity and risk of death. J Med Toxicol, 2011. 7(3): p. 205-12.

36. Parascandola, J., Dinitrophenol and bioenergetics: an historical perspective. Mol Cell Biochem, 1974. 5(1-2): p. 69-77.

37. De Felice, F.G. and S.T. Ferreira, Novel neuroprotective, neuritogenic and antiamyloidogenic properties of 2,4-dinitrophenol: the gentle face of Janus. IUBMB Life, 2006. 58(4): p. 185-91.

38. Harper, M.E., K. Green, and M.D. Brand, The efficiency of cellular energy transduction and its implications for obesity. Annu Rev Nutr, 2008. 28: p. 13-33.

39. Martineau, L.C., Large enhancement of skeletal muscle cell glucose uptake and suppression of hepatocyte glucose-6-phosphatase activity by weak uncouplers of oxidative phosphorylation. Biochim Biophys Acta, 2012. 1820(2): p. 133-50.

40. Bashan, N., et al., Mechanisms of adaptation of glucose transporters to changes in the oxidative chain of muscle and fat cells. Am J Physiol, 1993. 264(2 Pt 1): p. C43040.

41. Gates, A.C., et al., Respiratory uncoupling in skeletal muscle delays death and diminishes age-related disease. Cell Metab, 2007. 6(6): p. 497-505. 
42. Neschen, S., et al., Uncoupling protein 1 expression in murine skeletal muscle increases AMPK activation, glucose turnover, and insulin sensitivity in vivo. Physiol Genomics, 2008. 33(3): p. 333-40.

43. Kelley, D.E., et al., Skeletal muscle fatty acid metabolism in association with insulin resistance, obesity, and weight loss. Am J Physiol, 1999. 277(6 Pt 1): p. E1130-41.

44. Corpeleijn, E., et al., Impaired skeletal muscle substrate oxidation in glucoseintolerant men improves after weight loss. Obesity (Silver Spring), 2008. 16(5): p. 1025-32.

45. Salin, K., et al., Alteration of mitochondrial efficiency affects oxidative balance, development and growth in frog (Rana temporaria) tadpoles. J Exp Biol, 2012. 215(Pt 5): p. 863-9.

46. Mhatre, S.S., K.G. Chetty, and D.S. Pradhan, Uncoupling of oxidative phosphorylation in rat liver mitochondria following the administration of dimethyl sulphoxide. Biochem Biophys Res Commun, 1983. 110(1): p. 325-31. 


\section{CHAPTER 4}

\section{A genistein-enriched diet does not improve skeletal muscle oxidative capacity or prevent the transition toward advanced stages of insulin resistance in ZDF rats}

Bianca W.J. van Bree, Ellen Lenaers, Miranda Nabben, Jacco J. Briedé, Johanna A. Jörgensen, Gert Schaart, Patrick Schrauwen, Joris Hoeks, Matthijs K.C. Hesselink 


\section{ABSTRACT}

High levels of reactive oxygen species (ROS) and skeletal muscle mitochondrial dysfunction are associated with type 2 diabetes mellitus (T2DM). Genistein, a natural food compound mainly present in soybeans, is considered a potent antioxidant and to improve glucose homeostasis. However, its mechanism of action remains poorly understood. Here, we analyzed whether genistein could antagonize the progression of the hyperinsulinemic normoglycemic state (pre-diabetes) toward full-blown T2DM in Zucker Diabetic Fatty (ZDF) rats by decreasing mitochondrial oxidative stress and improving skeletal muscle oxidative capacity. ZDF rats were assigned to three groups: (1) lean control (CNTL), (2) fa/fa CNTL, and (3) fa/fa genistein (GEN). GEN animals were subjected to a $0.02 \%$ (w/w) genisteinenriched diet for 8 weeks, whereas CNTL rats received a standard diet. As assessed by intraperitoneal glucose tolerance tests, we show that genistein did not affect the overall response to a glucose challenge in ZDF rats $(P>0.05)$. In fact, genistein may exacerbate glucose intolerance as fasting glucose levels were higher in fa/fa GEN (17.6 $\pm 0.7 \mathrm{mM})$ compared with fa/fa CNTL animals $(14.9 \pm 1.4 \mathrm{mM}), P<0.05$. Oxidative stress and lipotoxicity, as established by electron spin resonance (ESR) spectroscopy, carbonylated protein content and UCP3 levels, remained unchanged upon dietary genistein supplementation $(P>0.05)$. Furthermore, respirometry measurements revealed no effects of genistein on mitochondrial function $(P>0.05)$. Therefore, dietary genistein supplementation did not improve glucose homeostasis, alleviate oxidative stress, or augment skeletal muscle metabolism in ZDF rats. Moreover, genistein feeding of hyperinsulinemic hyperglycemic ZDF rats did not rescue the progression toward advanced stages of insulin resistance. 


\section{INTRODUCTION}

A variety of natural dietary strategies have been examined to antagonize the progression of type 2 diabetes mellitus (T2DM) [1, 2]. As such, in type 2 diabetic humans the consumption of soy has been reported to have beneficial effects as it lowers fasting insulin levels and HOMA-IR, a surrogate marker for insulin resistance [3-6]. However, which constituent of soy mediates these protective effects and its related mechanisms remains unclear [7]. There are indications that the most abundant and active phytoestrogen in soy, genistein [8], could be beneficial in the management of T2DM by influencing skeletal muscle oxidative capacity. In vitro studies have shown that genistein can affect both glucose and lipid metabolism in skeletal muscle tissue [2]. Likewise, genistein stimulated glucose uptake in L6 myotubes independently of insulin under normoglycemic $(5.5 \mathrm{mM})$ and hyperglycemic $(25 \mathrm{mM})$ conditions in a dose-dependent manner [9]. Moreover, genistein was demonstrated to increase palmitate oxidation in C2C12 myotubes [10]. Animal models of T2DM also indicate that ingestion of a genistein-enriched diet could improve glucose homeostasis [11-14] by reducing hyperglycemia, circulating insulin levels $[12,15,16]$, and hepatic lipid accumulation $[17,18]$. Additionally, genistein has been reported to have antioxidant activities, either in vitro [19] or in vivo in liver and brain of insulin-resistant rodents [17, 20].

Although beneficial effects of genistein on glucose and fat homeostasis have been reported previously, the mechanisms underlying its beneficial effects remain poorly understood but include augmentation of mitochondrial oxidative capacity and ROS management. Interestingly, compromised mitochondrial function in skeletal muscle has been reported in models of insulin resistance and T2DM [21, 22] and may even precede overt T2DM. Since skeletal muscle glucose uptake is the central factor in maintenance of insulin sensitivity and development of T2DM, it will be important to elucidate the role of genistein in skeletal muscle mitochondrial capacity in a model of progressive insulin resistance. Therefore, the aim of this study was to investigate whether genistein influences glucose tolerance, and prevents or reduces the progression of the hyperinsulinemic normoglycemic state (pre-diabetes) towards full-blown T2DM by improving skeletal muscle oxidative capacity and alleviating oxidative stress. To this end, diabetes prone Zucker Diabetic Fatty (ZDF) rats were provided a genistein-enriched diet, starting at the age of 6 weeks, when rats were still normoglycemic. Our data show that consumption of a diet with $0.02 \%(\mathrm{w} / \mathrm{w})$ genistein does not affect whole-body glucose tolerance, mitochondrial skeletal muscle oxidative capacity or ROS production. Moreover, dietary genistein does not antagonize the pathogenesis of insulin resistance in ZDF rats. 


\section{MATERIALS AND METHODS}

\section{Animals}

Six-week old leptin receptor deficient ZDF rats (ZDF/Gmi, fa/fa, $n=12$ ) and their homozygote littermates (ZDF/Gmi, +/+ (lean), $n=12$ ) were purchased from Charles River (Maastricht, The Netherlands). Per genotype, the animals were housed in pairs in a controlled environment $\left(21^{\circ} \mathrm{C}\right)$ with a $12 \mathrm{~h}$ light : $12 \mathrm{~h}$ dark cycle. During the entire study, rats had ad libitum access to tap water and diet (Purina 5008; 16.7 energy percentage (En\%) fat, 56.4 En\% carbohydrates, and 26.8 En\% protein, Altromin Germany). Reported studies demonstrated that addition of genistein to the diet at a dose of $0.02 \%(w / w)$ positively affects lipid metabolism, reduces oxidative stress, and has anti-diabetic potential [10-12, 23, 24]. Therefore, from 6 weeks of age and onward, rats were fed ad libitum the standard Purina diet either with or without $0.02 \%(w / w)$ genistein (Shanghai Dnd Pharm-technology Co. Inc.) for the duration of 8 weeks ( $n=6$ per group). Body weight and food consumption was monitored weekly throughout this 8-week period of dietary intervention. Intraperitoneal glucose tolerance tests (IPGTT) were performed at 13 weeks of age. At the age of 14 weeks, rats were sacrificed by cervical dislocation after a short sedation with a mixture of $\mathrm{CO}_{2}$ and $\mathrm{O}_{2}$ $(4: 1)$. Subsequently, gastrocnemius muscles were rapidly dissected and partly ( 2 grams) directly used for the isolation of skeletal muscle mitochondria. Gastrocnemius muscle was also rapidly frozen for histology analyses and Western blotting assays in liquid $\mathrm{N}_{2}$-cooled isopentane and stored at $-80^{\circ} \mathrm{C}$. All protocols were approved by the Maastricht University animal ethics committee and complied with the principles of laboratory animal care.

\section{Glucose tolerance tests and plasma measurements}

For IPGTTs, rats were fasted for 4 hours and subsequently injected intraperitoneally with a glucose bolus ( $1 \mathrm{~g} / \mathrm{kg}$ body weight). Blood glucose levels were measured in vena saphena blood samples prior to $(t=0)$ and at $5,15,30,60,90$, and 120 minutes after the glucose administration using a glucose meter (LifeScan, Milpitas, CA, USA). Additionally, blood samples were collected in K-EDTA coated tubes, centrifuged at $1000 \mathrm{~g}$ for 10 minutes and plasma samples were frozen in liquid nitrogen and stored at $-80^{\circ} \mathrm{C}$ until analyses.

Leptin levels were established with a rat/mouse ELISA kit from Millipore (Millipore, Billerica, MA, USA).

\section{Mitochondrial isolation}

Within 30 seconds after sacrificing the animals, both gastrocnemius muscles were excised and processed on ice for mitochondrial isolation. In brief, the left gastrocnemius and the distal pieces of the right gastrocnemius muscle were immediately placed into ice-cold 
medium containing $100 \mathrm{mM}$ sucrose, $50 \mathrm{mM} \mathrm{KCl}, 20 \mathrm{mM} \mathrm{K}{ }^{+}$-TES, $1 \mathrm{mM}$ EDTA and $0.2 \%$ $(\mathrm{w} / \mathrm{v})$ bovine serum albumin (BSA) $(\mathrm{pH} 7,4$; with $\mathrm{KOH}$ ) and processed for mitochondrial isolation according to Nabben et al. [25]. The protein concentration in the mitochondrial pellet was measured using fluorescamine (Fluram ${ }^{\circledR}$, Fluka, Zwijndrecht, the Netherlands) with BSA as a standard [26]. Freshly isolated mitochondria were used immediately for electron spin resonance (ESR) spectroscopy and mitochondrial respirometry.

The medial part of the right gastrocnemius muscle was trimmed from visible fat and blood, embedded in Tissue-Tek (Sakura Finetek, Zoeterwoude, the Netherlands) and rapidly frozen in liquid nitrogen-cooled isopentane (2-methyl-butane, Fluka, Zwijndrecht, the Netherlands). Samples were stored at $-80^{\circ} \mathrm{C}$ until further analysis.

\section{Intramuscular lipid storage}

To quantify the neutral lipid storage in skeletal muscle, cryosections $(5 \mu \mathrm{m})$ of gastrocnemius muscles were stained with oil red $\mathrm{O}(\mathrm{ORO})$ as previously described [27]. ORO staining was combined with laminin staining (L-9393. Sigma, St Louis, USA) to visualize the cell membranes. Sections were examined using a Nikon E800 fluorescence microscope (Nikon, Amsterdam, The Netherlands). Digital images were captured and processed using Lucia G/F 5.49 image analysis software (Nikon, Dusseldorf, Germany). IMCL content was expressed per cell surface area and normalized to the lean control group.

\section{Reactive oxygen species (ROS) production}

Mitochondrial ROS production was measured using electron spin resonance (ESR) spectroscopy, as described earlier [28-30]. Briefly, freshly isolated mitochondria $(0.1 \mathrm{mg} / \mathrm{ml}$ protein) from gastrocnemius muscle were pre-incubated in mitochondrial respirometry medium (without malate), consisting of $100 \mathrm{mM}$ sucrose, $50 \mathrm{mM} \mathrm{KCl}, 20 \mathrm{mM} \mathrm{K}$-TES (pH 7.2), $2 \mathrm{mM} \mathrm{MgCl}$, $1 \mathrm{mM}$ EDTA, $4 \mathrm{mM} \mathrm{KH}_{2} \mathrm{PO}_{4}$, and $0.1 \%$ (w/v) bovine serum albumin $(B S A)$, at $37^{\circ} \mathrm{C}$ for 5 minutes. Subsequently, malate $(3 \mathrm{mM})$ and pyruvate $(5 \mathrm{mM})$ were added in the same concentrations as for the respirometry measurements. Charcoal purified 5,5-dimethyl-1-pyrroline N-oxide (DMPO) (100 mM; Sigma-Aldrich, St Louis, MO, USA) was added as a spintrap. Superoxide anion radical derived DMPO $-\mathrm{OH}$ signals were measured in triplicate (starting from the pre-incubation) on a Bruker EMX 1273 spectrometer [30]. Using the WIN-EPR spectrum quantification software (version 2.11, Bruker, Rheinstetten, Germany) spectra were quantified by summation of the peak heights from the four peaks of the DMPO $-\mathrm{OH}$ spectrum as previously described [31]. Values are expressed as percentage of the radical signal intensity of the lean control rats. 


\section{Mitochondrial respirometry}

Respiration rates were measured in freshly isolated mitochondria at $37^{\circ} \mathrm{C}$ using a twochambered Oxygraph (Oroboros Instruments, Innsbruck, Austria). Mitochondria (0.1 and $0.25 \mathrm{mg} / \mathrm{ml}$ mitochondrial protein for pyruvate and palmitoyl-CoA, respectively) were incubated in medium consisting of $100 \mathrm{mM}$ sucrose, $50 \mathrm{mM} \mathrm{KCl}, 20 \mathrm{mM} \mathrm{K}$-TES (pH 7.2), 2 $\mathrm{mM} \mathrm{MgCl}_{2}, 1 \mathrm{mM}$ EDTA, $4 \mathrm{mM} \mathrm{KH}_{2} \mathrm{PO}_{4}, 3 \mathrm{mM}$ malate and $0.1 \%(\mathrm{w} / \mathrm{v})$ bovine serum albumin (BSA). Pyruvate (5 mM) was used as a carbohydrate-derived substrate and carnitine (2 $\mathrm{mM})$ + palmitoyl-CoA $(50 \mu \mathrm{M})$ as fatty acid-derived substrate. After addition of substrate, maximally coupled (state 3) respiration was measured by adding ADP (450 $\mu \mathrm{M})$. Subsequently, basal (state 4) respiration was induced by the addition of the ATP-synthase inhibitor oligomycin ( $1 \mu \mathrm{g} / \mathrm{ml})$. Maximal mitochondrial respiration capacity (state $U$ respiration) was quantified by titrating the chemical uncoupler carbonyl cyanide $p$ trifluoromethoxyphenylhydrazone (FCCP). Mitochondrial respiration was expressed as nmol $\mathrm{O}_{2}$ per mg mitochondrial protein per minute.

\section{Western blot analysis}

To obtain a marker for mitochondrial density we used an antibody that selectively recognizes structural components of the electron transport chain and oxidative phosphorylation (rodent OXPHOS antibody cocktail, MS604; MitoSciences, Eugene, OR, USA). As lipotoxicity may contribute to the development of insulin resistance [32], especially under conditions of elevated production of reactive oxygen species [33,34] and UCP3 has been postulated to modulate lipotoxicity $[31,35,36]$, we also examined UCP3 protein levels. Hence, western blotting was performed in homogenates of the right gastrocnemius muscle as described previously [26, 37]. In short, standard SDS-PAGE blocking and incubation protocols were followed. Blots were incubated overnight at $4^{\circ} \mathrm{C}$ with primary antibodies against electron transport chain complexes proteins (OXPHOS) or UCP3 (rabbit polyclonal antibody to UCP3; ab3477; Abcam, Cambridge, UK). After incubation with the primary antibodies, appropriate secondary antibodies (Rockland Immunochemicals, TeBu, Heerhugowaard, The Netherlands) were applied. Both OXPHOS and UCP3 blots were visualized and quantified using the Odyssey Near Infrared Imager (Licor Biosciences, Westburg, Leusden, The Netherlands).

In addition, as a marker for oxidative damage, the amount of protein carbonyls in isolated mitochondrial fractions was quantified using an Oxyblot oxidized protein detection kit (Millipore, Billerica, MA, USA) according to Lenaers et al. [37]. All protein contents were expressed as arbitrary units (AU). 


\section{Statistics}

Results are expressed as means \pm SEM. For examining the effect of genistein, comparisons of data were made between lean CNTL, fa/fa CNTL, and fa/fa GEN groups through one-way ANOVA analyses followed by a Newman-Keuls posthoc test. To analyze the effect of genistein on glucose clearance during the IPGTT, mixed ANOVA analysis was performed with a Tukey posthoc test. Simple main effects were explored in order to determine the differences between groups at each time point. Per time point separate one-way ANOVAs were performed and multiple comparisons were obtained to establish the genistein effect per group at each time point. Statistical analyses were performed using GraphPad Prism 5.0a Macintosh Version. SPSS for Macinthosh 20.0 software was used for mixed ANOVA analysis. Statistical significance was set at $\mathrm{P}<0.05$.

\section{RESULTS}

\section{Animals}

Lean and fa/fa rats (aged 6 weeks) were placed on the standard Purina diet and body weight was monitored weekly throughout the 8-week intervention period. To study the effect of genistein on weight gain, a second group of fa/fa rats received the Purina diet supplemented with $0.02 \%(w / w)$ genistein. At 14 weeks of age, we observed that fa/fa rats consuming the genistein-enriched Purina diet had a similar body weight $(353 \pm 6 \mathrm{~g})$ in comparison to the $\mathrm{fa} / \mathrm{fa}$ rats on the standard Purina diet (344 $\pm 11 \mathrm{~g})$ (figure $1 \mathrm{~A})$. Genistein addition to the diet did not influence food consumption in the fa/fa animals (data not shown). In comparison to the lean control (CNTL) animals, the fa/fa rats had a $\sim 10 \%$ higher bodyweight $(P=0.001)$ (figure $1 \mathrm{~A})$ which can be attributed to the leptin receptor deficiency, resulting in a significantly higher food intake (39 g/day vs. $20 \mathrm{~g} /$ day respectively) despite high circulatory leptin levels in fa/fa animals (figure $1 \mathrm{~B}, \mathrm{P}<0.0001$ ).

Progression of insulin resistance during maturation of diabetes prone ZDF rats has previously been reported to occur in parallel with increased IMCL content [37, 38]. Moreover, genistein has been suggested to promote lipid oxidation [39], hence we also assessed the effect of genistein on skeletal muscle fat content (figure 1C). In line with previous observations we observed profoundly higher IMCL levels in fa/fa CNTL and fa/fa GEN rats than in lean CNTL rats (figure $1 \mathrm{D}, \mathrm{P}<0.05$ ). Dietary supplementation of genistein, however, did not affect IMCL content significantly.

\section{Whole body glucose tolerance}

The fa/fa ZDF rat is a recognized model of progressive insulin resistance during maturation [38]. At 6 weeks of age fa/fa rats are known to be hyperinsulinemic and normoglycemic, 
reflecting a pre-diabetic state, whereas at 14 weeks of age fa/fa rats suffer from severe hyperglycemia [37]. As cell studies indicate that genistein promotes glucose uptake in myocytes in vitro [9], we studied the hypothesis that genistein would also promote glucose tolerance in vivo. Hence we tested if consumption of dietary genistein during maturation affects whole-body glucose tolerance by performing i.p. glucose tolerance tests (IPGTT) in rats at 13 weeks of age.

A
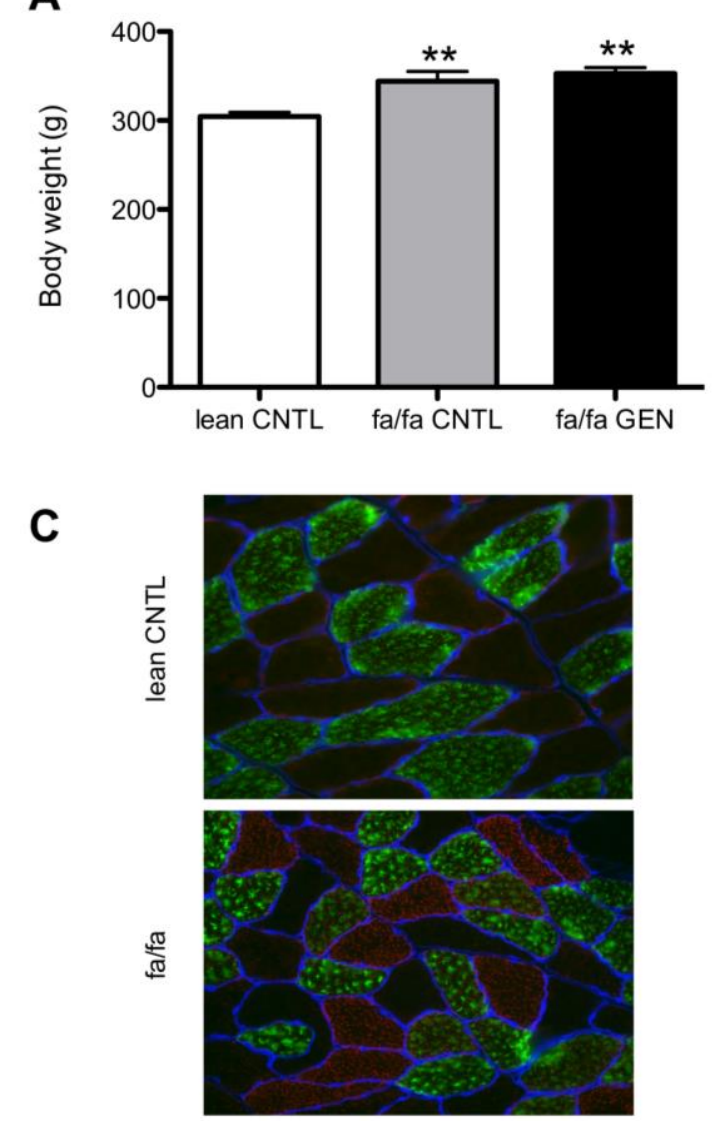

B

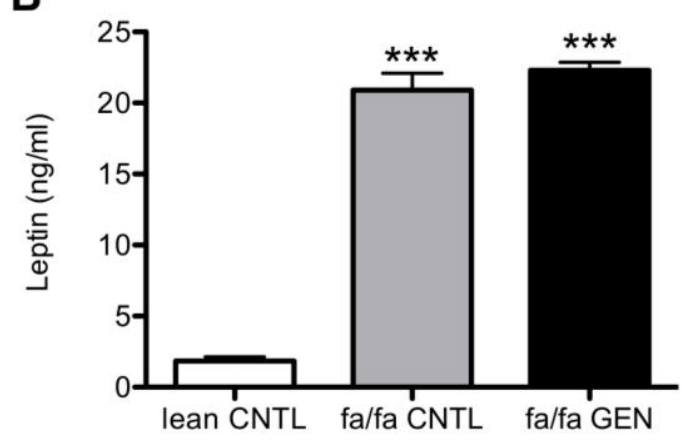

D

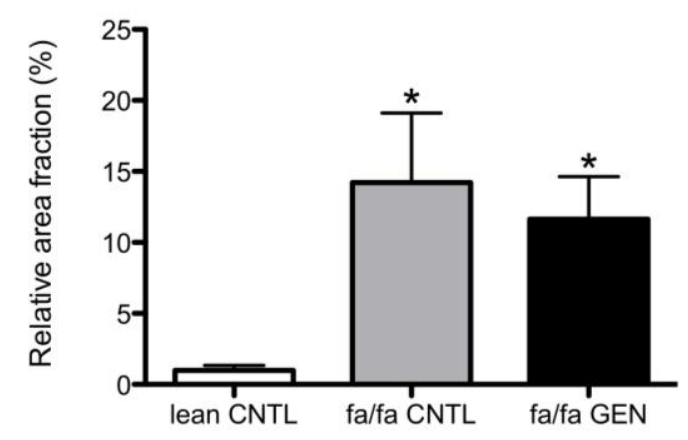

Figure 1. Dietary genistein consumption does not affect body weight or intramyocellular lipid content during maturation of ZDF rats. (A) Body weights and (B) plasma leptin levels of rats at the end of the 8-week dietary intervention. (C) Representative microscope images of ORO staining in gastrocnemius muscle sections from lean CNTL (upper panel) and fa/fa CNTL (lower panel) rats. (D) Quantification of IMCL content in gastrocnemius muscle expressed as percentage relative area fraction. The relative area fraction of IMCL content in lean CNTL rats was set to $1.0 \%$. ${ }^{*}: P<0.05$ vs. lean $C N T L,{ }^{* *}: P<0.01$ vs. lean $C N T L,{ }^{* *}: P<0.0001$ vs. lean $C N T L$.

As anticipated, fasting blood glucose levels were significantly higher in $\mathrm{fa} / \mathrm{fa}$ rats compared to lean rats $(16.4 \pm 0.8 \mathrm{mM}$ and $5.5 \pm 0.2 \mathrm{mM}$ respectively, $\mathrm{P}<0.0001$, figure $2 \mathrm{~A}$ ). Interestingly, and in contrast to what was anticipated based upon studies by others [9, 13, 15], we observed that dietary supplementation of genistein resulted in significantly higher fasting blood glucose levels in genistein treated fa/fa rats (fa/fa GEN, 17.6 $\pm 0.7 \mathrm{mM}$ ) than in $\mathrm{fa} / \mathrm{fa}$ rats fed the control diet (fa/fa CNTL, 14.9 $\pm 1.4 \mathrm{mM}$ ), $\mathrm{P}<0.05$, figure $2 \mathrm{~A}$ ). 
Next we analyzed the blood glucose response to an intraperitoneal glucose challenge (IPGTT). An interaction effect was found between time and intervention on blood glucose levels $(\mathrm{P}<0.0001$, figure $2 \mathrm{~B})$, indicating differences in rise and clearance of plasma glucose between groups upon the i.p. glucose bolus. The glucose intolerant state of the fa/fa rats was confirmed by the significantly higher blood glucose concentration at all time points during the IPGTT in both fa/fa groups compared to the lean CNTL group (One-way ANOVAs $p<0.0001$, figure $2 \mathrm{~B}$ ). Although the overall response (as is reflected by the $\mathrm{A} A \cup C$ ) to an intraperitoneal glucose load was not affected by genistein, glucose levels at $60(\mathrm{P}=0.002)$ and 120 minutes

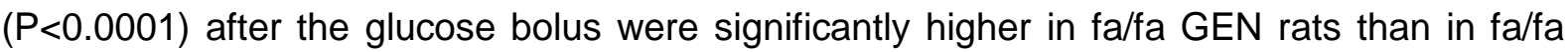
CNTL rats (figure $2 \mathrm{~B}$ ). Despite these unfavorable effects of genistein on fasting glucose and glucose levels at $t=60 \mathrm{~min}$ and $t=120 \mathrm{~min}$, the incremental area under the time-glucose curves (figure $2 \mathrm{C}$ ) was not significantly different between fa/fa rats consuming the control diet and genistein-treated; $709 \pm 8$ and $765 \pm 3$ in fa/fa CNTL and fa/fa GEN rats respectively (figure 2C).

A

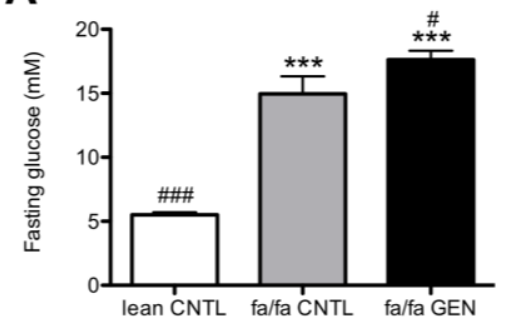

B

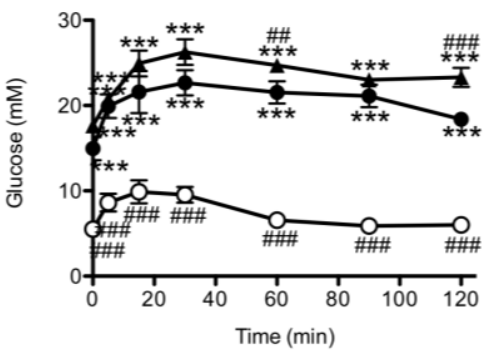

C

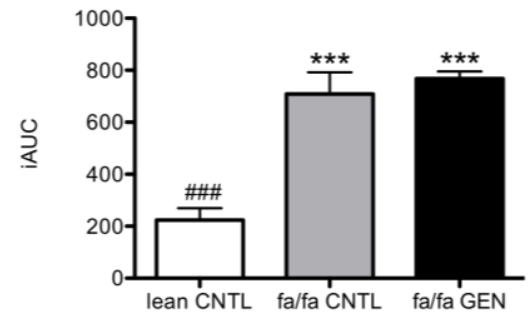

Figure 2. Dietary genistein consumption does not improve whole-body glucose tolerance in fa/fa rats at the age of 13 weeks. (A) 4-hr fasting glucose levels (mM) at T=0 of the IPGTT after 7 weeks of dietary intervention. (B) Glucose tolerance curves of lean CNTL (open circles), fa/fa CNTL (closed circles) and fa/fa GEN (closed triangles) treated animals. (C) Analysis of the incremental area under the curve (iAUC) in groups as indicated. ***: $P<0.0001$ vs. lean CNTL, \#: $P<0.05$ vs. fa/fa CNTL \#\#: $P<0.01$ vs. fa/fa CNTL, \#\#\#: $P<0.0001$ vs. fa/fa CNTL.

\section{Oxidative stress}

Genistein has been reported to have antioxidant activity in vitro as well as in vivo in liver and brain tissues of insulin resistant rodents $[17,19,20]$. To explore whether genistein was able to also blunt mitochondrial ROS production in skeletal muscle, we measured superoxide anion radical production in a direct manner in isolated mitochondria from gastrocnemius muscles, at a high proton gradient (state 4 conditions, by ESR spectroscopy). This direct measure of superoxide production, however, revealed similar levels of skeletal muscle mitochondrial superoxide production in genistein-treated animals compared to animals on a control diet (figure $3 \mathrm{~A}, \mathrm{P}=0.15$ ). Thus, no differences were found between lean CNTL vs. fa/fa or between fa/fa CNTL and fa/fa GEN animals. 
Next to mitochondrial ROS production, we also measured oxidative protein damage by quantifying carbonylated protein content through Oxyblot. In accordance with similar levels of mitochondrial superoxide generation, carbonylated protein content remained unaffected upon genistein-treatment $(0.93 \pm 0.09$ vs. $0.98 \pm 0.10$ in fa/fa CNTL vs. fa/fa GEN rats respectively) $(P=0.89$, figure $3 \mathrm{~B})$. Moreover, the fa/fa and lean CNTL had similar levels of carbonylated proteins. Jointly, these data suggest that genistein does not affect the antioxidant capacity in rat skeletal muscle.

\section{Mitochondrial uncoupling}

UCP3 has been proposed to modulate lipotoxicity by alleviating proton gradient built-up [31, $35,36]$ thereby blunting the production of superoxide. This, along with the observation in $\mathrm{C} 2 \mathrm{C} 12$ cells that genistein induces gene expression of UCP3 in $\mathrm{C} 2 \mathrm{C} 12$ myotubes [10], prompted us to measure UCP3 protein content. UCP3 protein content, however, was not affected by genistein ( $P=0.98$, figure $3 C$ ).
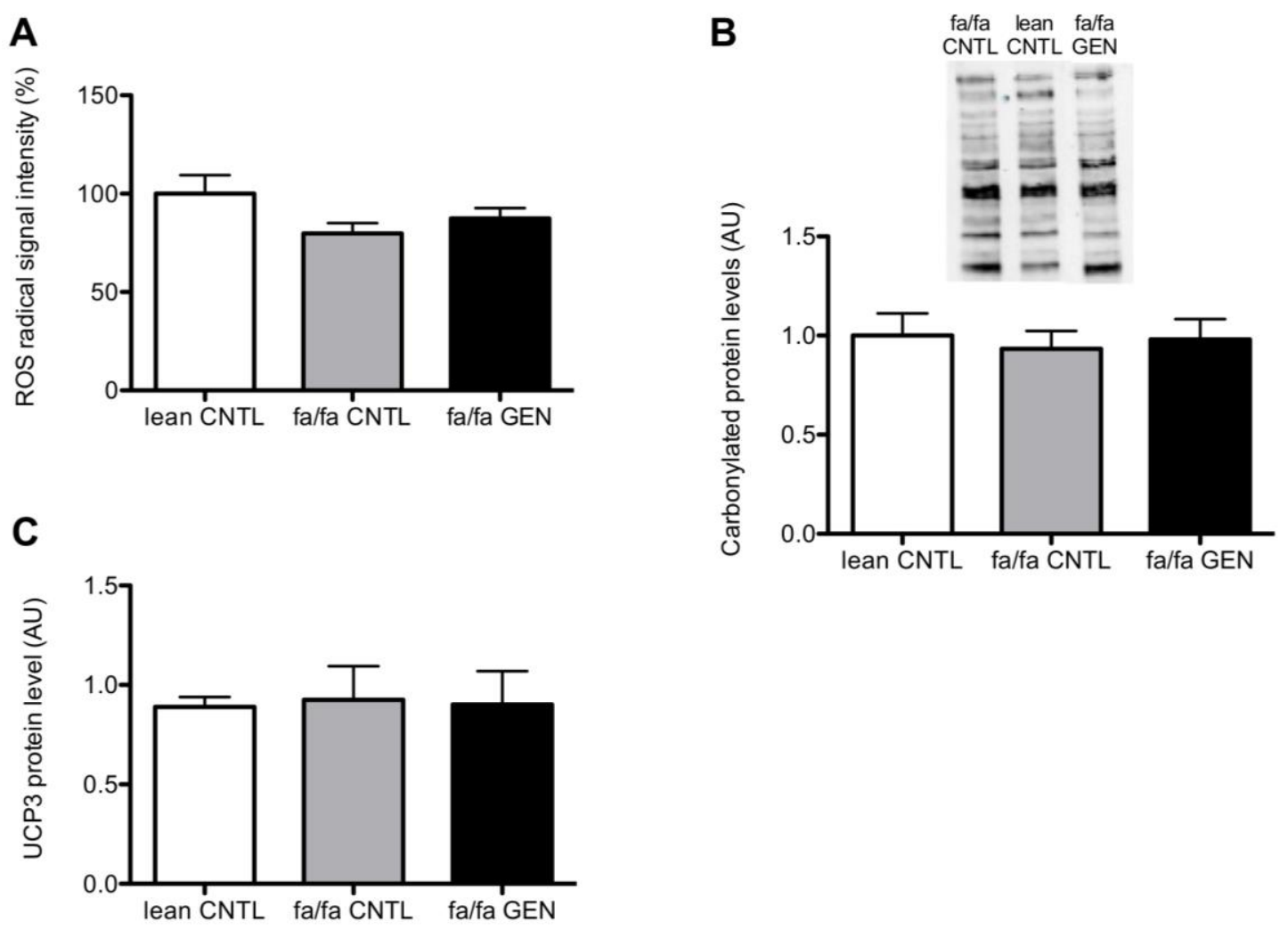

Figure 3. 8 weeks of genistein feeding had no effects on skeletal muscle oxidative stress. (A) Superoxide anion radical production on malate with pyruvate as substrates was measured using ESR spectroscopy. The ROS radical signal intensity of the lean CNTL rats was set to $100 \%$. (B) Quantification of oxidative stress in gastrocnemius muscles by analysis of carbonylated protein levels with Oxyblot. The carbonylated protein content in skeletal muscle of lean CNTL rats was set to 1.0 (AU). (C) Protein UCP3 expression levels in gastrocnemius muscle quantified by western blot analysis (AU). 


\section{Skeletal muscle mitochondrial density and function}

Subsequently, we analyzed the putative effect of genistein on skeletal muscle mitochondrial density and function. Mitochondrial density was estimated by quantifying protein levels of the structural components of the complexes involved in oxidative phosphorylation (OXPHOS) (Figure 4A). Protein content of OXPHOS complexes was comparable in all groups (One-way ANOVAs, complex I $P=0.2$, complex II $P=0.39$, complex III $P=0.71$, or complex $\vee P=0.99$ ), regardless of the genotype (figure 4B). Also the sum of OXPHOS complexes was not different between groups ( $P=0.49$, figure $4 C$ ). Thus, the mitochondrial density seemed not significantly affected by genistein administration.

Intrinsic mitochondrial respiratory capacity on either carbohydrate- or fatty acidderived substrates was determined by measuring mitochondrial respiration upon either pyruvate or palmitoyl-CoA with carnitine. However, neither genistein treatment nor genotype significantly affected either maximal coupled (ADP-driven respiration, state 3$)(P=0.76)$ or uncoupled (FCCP-stimulated respiration, state $U)(P=0.68)$ mitochondrial respiration on pyruvate (figure $5 \mathrm{~A}-\mathrm{B})$ or palmitoyl-CoA with carnitine $(P=0.62$ and $P=0.29$ for state 3 and state $U$ respectively) (figure $5 C-D$ ). These data indicate that dietary genistein did neither affect mitochondrial density nor intrinsic mitochondrial function.

A

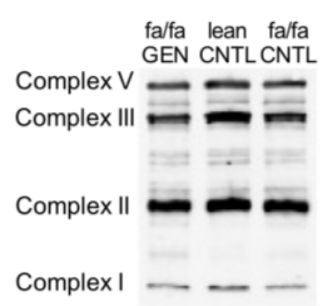

B

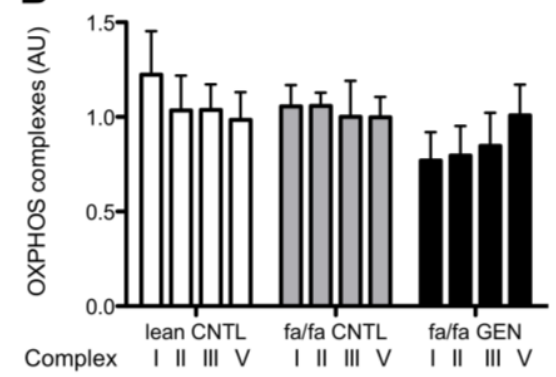

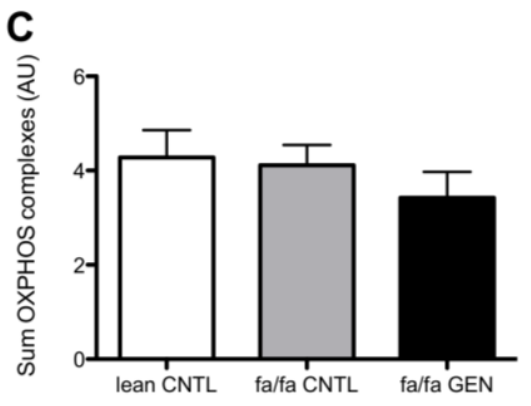

Figure 4. Similar mitochondrial densities in 14-week-old lean CNTL, fa/fa CNTL, and fa/fa GEN groups as were estimated by quantifying complexes I, II, III, and V of the mitochondrial respiratory chain (OXPHOS). (A) Representative western blot. (B) Quantification of western blot analysis of protein expression of OXPHOS complexes (AU) in gastrocnemius muscle homogenates and (C) sum of OXPHOS complexes (AU) in lean and fa/fa rats. 
A

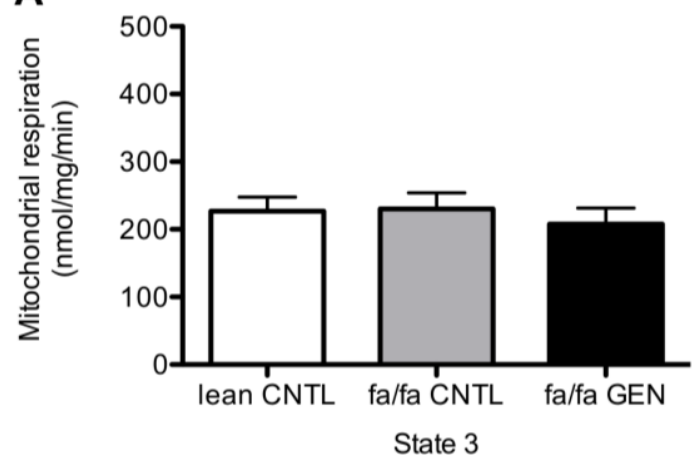

C

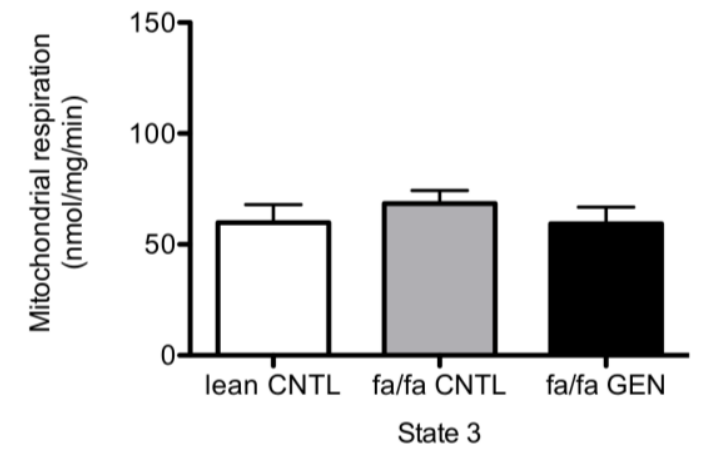

B

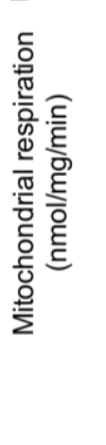

D

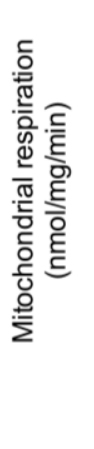

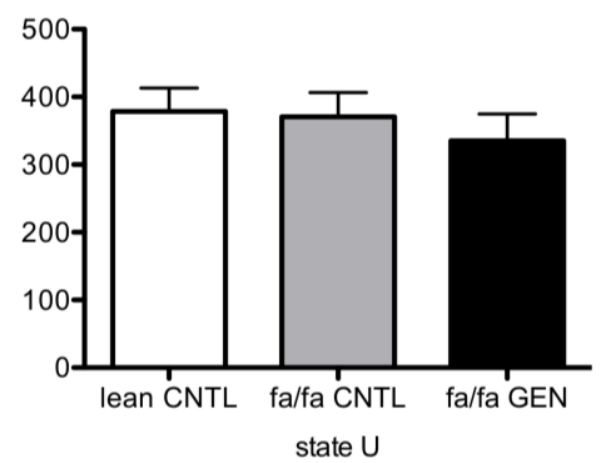

state $U$

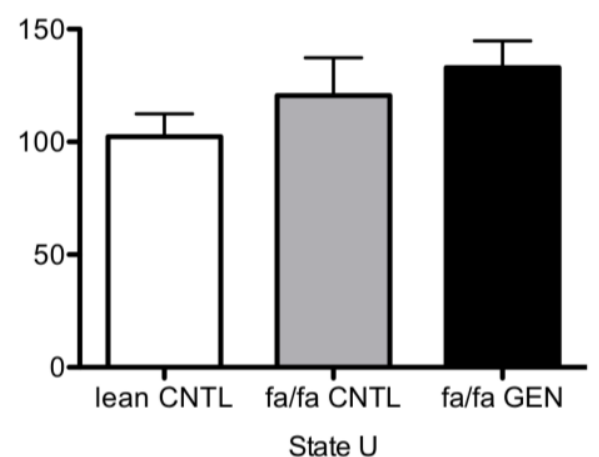

Figure 5. Oxidative capacity of isolated mitochondria from gastrocnemius muscle is not affected upon genistein consumption. (A) Coupled (ADP-driven) mitochondrial state 3 respiration upon pyruvate, (B) Uncoupled (FCCPstimulated) state $U$ respiration on pyruvate. (C) State 3 on palmitoyl-CoA and D) State $U$ on palmitoyl-CoA in lean and $\mathrm{fa} / \mathrm{fa}$ rats at 14 weeks of age.

\section{DISCUSSION}

Genistein, a natural food compound mainly present in soybeans, has been shown to alleviate symptoms associated with T2DM. Nevertheless, the exact mechanism(s) underlying effects of genistein on glucose homeostasis under diabetic conditions remain poorly understood. In myotubes, genistein has been shown to improve glucose uptake [9] and fatty acid handling [10], implying improved oxidative capacity in muscle. In rodent models of T2DM, genistein has been reported to improve glucose homeostasis [11-14] and attenuate ROS-generation $[17,20]$. Because skeletal muscle plays a central role in the maintenance of insulin sensitivity [40], and muscle mitochondrial dysfunction is associated with insulin resistance and T2DM $[21,22]$, we here examined whether genistein influences muscle mitochondrial function and superoxide production in a rat model of progressive insulin resistance. We hypothesized that dietary supplementation of genistein inhibits the transition of the hyperinsulinemic normoglycemic state (pre-diabetes) towards full blown T2DM by improving skeletal muscle mitochondrial oxidative capacity. However, we found no evidence for genistein supplementation to improve whole-body glucose tolerance, skeletal muscle oxidative capacity, or ROS-induced stress during the maturation of ZDF rats. Therefore, our data 
argue against a beneficial role of genistein in the prevention of the development of advanced insulin resistance. In fact, increased fasting glucose levels in genistein-supplemented rats and elevated glucose levels at several time points after and oral glucose load may even be interpreted as unwarranted.

The ZDF rat is a well-known model of progressive insulin resistance [38]. As anticipated, we found that fa/fa rats had higher leptin levels, food intake, and body weight in comparison to lean rats. At 14 weeks of age fa/fa rats had significantly elevated fasting glucose levels and muscle fat content compared to lean rats, implying that fa/fa rats had developed an advanced state of insulin resistance indeed. In addition, no major differences were found in mitochondrial function upon either pyruvate or palmitoyl-CoA with carnitine between mature $\mathrm{fa} / \mathrm{fa}$ and lean rats, which is consistent with findings in previous studies using the same rat model $[37,41]$. Hence, studying effects of genistein on glucose homeostasis and muscle oxidative capacity in fa/fa ZDF rats during maturation could provide more insight in the potential of genistein to inhibit the transition from a pre-diabetic state toward advanced stages of insulin resistance.

Interestingly, we found that after 8 weeks dietary intervention, fa/fa rats that consumed the genistein-enriched diet (fa/fa GEN) had similar body weights compared with the untreated fa/fa (fa/fa CNTL) rats. Also food intake and IMCL content were not different upon genistein. However, genistein had a negative rather than a positive effect on hyperglycemia as fasting glucose levels were slightly elevated in fa/fa GEN rats compared with the fa/fa CNTL rats. Also, blood glucose levels at 60 and 120 minutes after a glucose bolus were slightly higher in fa/fa GEN rats compared with fa/fa CNTL rats. Thus, unexpectedly, our data show a more unfavorable rather than favorable effect of dietary genistein on glucose homeostasis under conditions of insulin resistance. A previous study using nongenetic obese diabetic mice that were generated by high fat feeding and streptozotocin injections, also examined the effect of genistein on whole-body glucose homeostasis [15]. Whereas there were also no effects of genistein on body weight or fat deposition in these mice, hyperglycemia was blunted and glucose tolerance improved upon $0.025 \%$ dietary genistein intake [15]. Importantly, streptozotocin injections rendered these mice diabetic due to compromised pancreatic $\beta$-cell function. Thus, the mechanism underlying insulin resistance in streptozotocin diabetic mice is essentially different from the fa/fa ZDF model, where muscle insulin resistance appears to be the primary defect. The essential difference in the underlying mechanism may explain the discrepancy between the beneficial effects of genistein on glucose tolerance in streptozotocin treated mice vs. our observations in fa/fa rats. Additionally, the actions of genistein (and other isoflavones), appear to depend on the complex interaction of several factors, including the its duration and 
concentration of dosage regimens, but also age and sex of the individual have been reported to affect the action of genistein [42].

Although genistein had no beneficial effects on whole-body glucose homeostasis, genistein could still have an effect on substrate metabolism at the level of skeletal muscle. Type 2 diabetes patients have been characterized by increased levels of skeletal muscle fat content [43-45] accompanied by excessive ROS generation and oxidative stress [34, 46, 47]. As a consequence, enhanced lipid peroxidation leads to increased production of lipotoxic byproducts that could negatively affect muscle substrate metabolism [32, 48], and subsequently, contribute to the development of T2DM [46, 49].

Since recent data point toward a beneficial role of genistein against ROS [17-20], we examined the effect of genistein on mitochondrial superoxide production and oxidative damage in skeletal muscle under insulin resistance conditions. To the best of our knowledge, this is the first study demonstrating that genistein supplementation does not directly affect skeletal muscle mitochondrial superoxide anion radical formation as assessed by ESR. This was accompanied by lack of effect of genistein treatment on oxidative stress levels as was reflected by comparable levels of carbonylated proteins [50]. Analogous to our data on oxidative stress, UCP3 protein levels, another postulated protective mechanism against lipotoxicity [31, 35, 36] suggested to be activated by genistein [10], remain unaffected. It has been reported that effects of genistein on oxidative stress are less pronounced under insulin resistance conditions. In this context, in human umbilical vein endothelial cells, genistein had protective effects against ROS-induced apoptosis and inhibition of cell proliferation under normal glucose conditions (5 $\mathrm{mM}$ ) [51]. Though, these protective effects were less effective at high glucose levels $(25 \mathrm{mM})$ mimicking diabetic conditions [51]. These data along with our findings in the present study imply that genistein alone does not effectively lower oxidative stress in skeletal muscle under conditions of insulin resistance in ZDF rats.

Nevertheless, it is important to note that our focus was on mitochondrial ROS production and markers of ROS-induced damage in skeletal muscle. In livers of diet-induced insulin-resistant rats it has been reported that a dose of $1 \mathrm{mg} / \mathrm{kg}$ genistein via oral gavage did prevent oxidative damage [17]. Additionally, in a mouse model of focal cerebral ischemia injury, a genistein dose of half the concentration of what was used in the present study (2.5$10 \mathrm{mg} / \mathrm{kg}$ vs. $15-20 \mathrm{mg} / \mathrm{kg}$ per day respectively) could decrease ROS generation directly as was shown by reduced $\mathrm{H}_{2} \mathrm{O}_{2}$ levels [52]. Tissue-specific effects of genistein on oxidative stress could be induced due to the presence of tissue-specific estrogen receptor (ER) $\alpha / E R \beta$ ratios [53] and tissue differences may help to understand the aberrant differences between these studies and ours.

To elucidate the effect of genistein on skeletal muscle oxidative capacity in more detail, we measured mitochondrial density and function. Comparable OXPHOS levels 
between fa/fa CNTL and fa/fa GEN demonstrate that consuming a diet enriched with $0.02 \%$ (w/w) genistein during 8 weeks did not influence mitochondrial density in 14-week-old ZDF rats. Also, genistein supplementation did not improve intrinsic mitochondrial function. This finding was displayed by comparable levels of maximal coupled (ADP-driven) and uncoupled (FCCP-stimulated) mitochondrial respiration on either palmitoyl-CoA or pyruvate, in $\mathrm{fa} / \mathrm{fa}$ CNTL and fa/fa GEN groups. Thus, genistein supplementation during the development of advanced stages of insulin resistance does not affect mitochondrial density, or influence intrinsic mitochondrial function. Our data on mitochondrial density and function match our finding that genistein did not reduce oxidative stress under diabetic conditions. Therefore, our data demonstrate that genistein is not capable to blunt or alleviate the transition of the hyperinsulinemic normoglycemic state towards advanced insulin resistance by improving skeletal muscle mitochondrial oxidative capacity in ZDF rats.

In conclusion, we here studied the effects of genistein on glucose homeostasis and skeletal muscle metabolism in a rat model of progressive insulin resistance and development of T2DM. Our data show that dietary supplementation of $0.02 \%(w / w)$ genistein had no beneficial effects on whole-body glucose tolerance, skeletal muscle oxidative stress, or intrinsic mitochondrial function in ZDF rats. Since skeletal muscle is an important side of insulin resistance and T2DM development, we suggest that dietary supplementation of pure genistein does not alleviate symptoms associated with T2DM progression. Moreover, we here showed that genistein feeding of hyperinsulinemic hyperglycemic ZDF rats does not prevent or inhibit the transition from a prediabetic state toward advanced insulin resistance and development of T2DM. In fact, we even demonstrated that under diabetic conditions, genistein could give unfavorable effects on glucose homeostasis.

\section{Acknowledgements}

The work of E. Lenaers has been supported by a grant from the School of Life Sciences, M.K.C. Hesselink was supported by a VIDI grant for innovative research (917.66.359).

\section{REFERENCES}

1. Abete, I., et al., Obesity and metabolic syndrome: potential benefit from specific nutritional components. Nutr Metab Cardiovasc Dis, 2011. 21 Suppl 2: p. B1-15.

2. Cederroth, C.R. and S. Nef, Soy, phytoestrogens and metabolism: A review. Mol Cell Endocrinol, 2009. 304(1-2): p. 30-42.

3. Fujimoto, W.Y., et al., Glucose intolerance and diabetic complications among Japanese-American women. Diabetes Res Clin Pract, 1991. 13(1-2): p. 119-29.

4. Squadrito, F., et al., Genistein in the metabolic syndrome: results of a randomized clinical trial. J Clin Endocrinol Metab, 2013. 98(8): p. 3366-74.

5. Jayagopal, V., et al., Beneficial effects of soy phytoestrogen intake in postmenopausal women with type 2 diabetes. Diabetes Care, 2002. 25(10): p. 1709-14.

6. Bhathena, S.J. and M.T. Velasquez, Beneficial role of dietary phytoestrogens in obesity and diabetes. Am J Clin Nutr, 2002. 76(6): p. 1191-201. 
7. Zimmermann, C., et al., Prevention of diabetes in $\mathrm{db} / \mathrm{db}$ mice by dietary soy is independent of isoflavone levels. Endocrinology, 2012. 153(11): p. 5200-11.

8. Mantovani, D., et al., Chromatographic quantification of isoflavone content from soy derivates using HPLC technique. J Chromatogr Sci, 2009. 47(9): p. 766-9.

9. Ha, B.G., et al., Regulatory mechanism for the stimulatory action of genistein on glucose uptake in vitro and in vivo. J Nutr Biochem, 2012. 23(5): p. 501-9.

10. Palacios-Gonzalez, B., et al., Genistein stimulates fatty acid oxidation in a leptin receptor-independent manner through the JAK2-mediated phosphorylation and activation of AMPK in skeletal muscle. Biochim Biophys Acta, 2013. 1841(1): p. 132140.

11. Mezei, O., et al., Soy isoflavones exert antidiabetic and hypolipidemic effects through the PPAR pathways in obese Zucker rats and murine RAW 264.7 cells. J Nutr, 2003. 133(5): p. 1238-43.

12. Ae Park, S., et al., Genistein and daidzein modulate hepatic glucose and lipid regulating enzyme activities in C57BL/KsJ-db/db mice. Life Sci, 2006. 79(12): p. 120713.

13. Lee, J.S., Effects of soy protein and genistein on blood glucose, antioxidant enzyme activities, and lipid profile in streptozotocin-induced diabetic rats. Life Sci, 2006. 79(16): p. 1578-84.

14. Davis, J., et al., Soy protein influences the development of the metabolic syndrome in male obese ZDFxSHHF rats. Horm Metab Res, 2005. 37(5): p. 316-25.

15. Fu, Z., et al., Genistein ameliorates hyperglycemia in a mouse model of nongenetic type 2 diabetes. Appl Physiol Nutr Metab, 2012. 37(3): p. 480-8.

16. Ali, A.A., et al., Modulation of carbohydrate metabolism and peptide hormones by soybean isoflavones and probiotics in obesity and diabetes. J Nutr Biochem, 2005. 16(11): p. 693-9.

17. Mohamed Salih, S., et al., Genistein improves liver function and attenuates nonalcoholic fatty liver disease in a rat model of insulin resistance. J Diabetes, 2009. 1(4): p. 278-87.

18. Tovar, A.R., et al., Soy protein reduces hepatic lipotoxicity in hyperinsulinemic obese Zucker fa/fa rats. J Lipid Res, 2005. 46(9): p. 1823-32.

19. Vedavanam, K., et al., Antioxidant action and potential antidiabetic properties of an isoflavonoid-containing soyabean phytochemical extract (SPE). Phytother Res, 1999. 13(7): p. 601-8.

20. Valsecchi, A.E., et al., The soy isoflavone genistein reverses oxidative and inflammatory state, neuropathic pain, neurotrophic and vasculature deficits in diabetes mouse model. Eur J Pharmacol, 2011. 650(2-3): p. 694-702.

21. Petersen, K.F., et al., Impaired mitochondrial activity in the insulin-resistant offspring of patients with type 2 diabetes. N Engl J Med, 2004. 350(7): p. 664-71.

22. Chow, L., A. From, and E. Seaquist, Skeletal muscle insulin resistance: the interplay of local lipid excess and mitochondrial dysfunction. Metabolism, 2010. 59(1): p. 70-85.

23. Davis, J., et al., Soy protein and isoflavones influence adiposity and development of metabolic syndrome in the obese male ZDF rat. Ann Nutr Metab, 2007. 51(1): p. 42-52.

24. Lee, Y.M., et al., Effects of dietary genistein on hepatic lipid metabolism and mitochondrial function in mice fed high-fat diets. Nutrition, 2006. 22(9): p. 956-64.

25. Nabben, M., et al., Uncoupled respiration, ROS production, acute lipotoxicity and oxidative damage in isolated skeletal muscle mitochondria from UCP3-ablated mice. Biochim Biophys Acta, 2011. 1807(9): p. 1095-105.

26. Schrauwen, P., et al., Uncoupling protein 3 as a mitochondrial fatty acid anion exporter. FASEB J, 2003. 17(15): p. 2272-4.

27. Koopman, R., G. Schaart, and M.K. Hesselink, Optimisation of oil red O staining permits combination with immunofluorescence and automated quantification of lipids. Histochem Cell Biol, 2001. 116(1): p. 63-8.

28. Hoeks, J., et al., Mitochondrial function, content and ROS production in rat skeletal muscle: effect of high-fat feeding. FEBS Lett, 2008. 582(4): p. 510-6. 
29. Nabben, M., et al., The effect of UCP3 overexpression on mitochondrial ROS production in skeletal muscle of young versus aged mice. FEBS Lett, 2008. 582(30): p. 4147-52.

30. Briede, J.J., et al., The presence of the cag pathogenicity island is associated with increased superoxide anion radical scavenging activity by Helicobacter pylori. FEMS Immunol Med Microbiol, 2005. 44(2): p. 227-32.

31. Nabben, M., et al., Significance of uncoupling protein 3 in mitochondrial function upon mid- and long-term dietary high-fat exposure. FEBS Lett, 2011. 585(24): p. 4010-7.

32. Russell, A.P., et al., Lipid peroxidation in skeletal muscle of obese as compared to endurance-trained humans: a case of good vs. bad lipids? FEBS Lett, 2003. 551(1-3): p. 104-6.

33. Schrauwen, P., et al., Mitochondrial dysfunction and lipotoxicity. Biochim Biophys Acta, 2010. 1801(3): p. 266-71.

34. Houstis, N., E.D. Rosen, and E.S. Lander, Reactive oxygen species have a causal role in multiple forms of insulin resistance. Nature, 2006. 440(7086): p. 944-8.

35. Aguer, C., et al., Muscle uncoupling protein 3 overexpression mimics endurance training and reduces circulating biomarkers of incomplete beta-oxidation. FASEB J, 2013. 27(10): p. 4213-25.

36. Toime, L.J. and M.D. Brand, Uncoupling protein-3 lowers reactive oxygen species production in isolated mitochondria. Free Radic Biol Med, 2010. 49(4): p. 606-11.

37. Lenaers, E., et al., Adaptations in mitochondrial function parallel, but fail to rescue, the transition to severe hyperglycemia and hyperinsulinemia: a study in Zucker diabetic fatty rats. Obesity (Silver Spring, Md.), 2010. 18(6): p. 1100-1107.

38. Etgen, G. and B. Oldham, Profiling of Zucker diabetic fatty rats in their progression to the overt diabetic state. Metabolism: clinical and experimental, 2000. 49(5): p. 684-688.

39. Naaz, A., et al., The soy isoflavone genistein decreases adipose deposition in mice. Endocrinology, 2003. 144(8): p. 3315-20.

40. Shulman, G.I., et al., Quantitation of muscle glycogen synthesis in normal subjects and subjects with non-insulin-dependent diabetes by 13C nuclear magnetic resonance spectroscopy. N Engl J Med, 1990. 322(4): p. 223-8.

41. De Feyter, H.M., et al., Increased intramyocellular lipid content but normal skeletal muscle mitochondrial oxidative capacity throughout the pathogenesis of type 2 diabetes. FASEB J, 2008. 22(11): p. 3947-55.

42. Orgaard, A. and L. Jensen, The effects of soy isoflavones on obesity. Exp Biol Med (Maywood), 2008. 233(9): p. 1066-80.

43. Pan, D.A., et al., Skeletal muscle triglyceride levels are inversely related to insulin action. Diabetes, 1997. 46(6): p. 983-8.

44. Perseghin, G., et al., Intramyocellular triglyceride content is a determinant of in vivo insulin resistance in humans: a $1 \mathrm{H}-13 \mathrm{C}$ nuclear magnetic resonance spectroscopy assessment in offspring of type 2 diabetic parents. Diabetes, 1999. 48(8): p. 1600-6.

45. Krssak, M., et al., Intramyocellular lipid concentrations are correlated with insulin sensitivity in humans: a 1H NMR spectroscopy study. Diabetologia, 1999. 42(1): p. 113-6.

46. Anderson, E.J., et al., Mitochondrial $\mathrm{H} 2 \mathrm{O} 2$ emission and cellular redox state link excess fat intake to insulin resistance in both rodents and humans. J Clin Invest, 2009. 119(3): p. 573-81.

47. Brand, M.D., et al., Oxidative damage and phospholipid fatty acyl composition in skeletal muscle mitochondria from mice underexpressing or overexpressing uncoupling protein 3. Biochem J, 2002. 368(Pt 2): p. 597-603.

48. Furukawa, S., et al., Increased oxidative stress in obesity and its impact on metabolic syndrome. J Clin Invest, 2004. 114(12): p. 1752-61.

49. Lee, H.Y., et al., Targeted expression of catalase to mitochondria prevents ageassociated reductions in mitochondrial function and insulin resistance. Cell Metab, 2010. 12(6): p. 668-74.

50. Dalle-Donne, I., et al., Protein carbonyl groups as biomarkers of oxidative stress. Clin Chim Acta, 2003. 329(1-2): p. 23-38. 
51. Xu, S.Z., et al., Multiple mechanisms of soy isoflavones against oxidative stressinduced endothelium injury. Free Radic Biol Med, 2009. 47(2): p. 167-75.

52. Qian, Y., et al., Neuroprotection by the soy isoflavone, genistein, via inhibition of mitochondria-dependent apoptosis pathways and reactive oxygen induced-NF-kappaB activation in a cerebral ischemia mouse model. Neurochem Int, 2012. 60(8): p. 759-67.

53. Nadal-Serrano, M., et al., Genistein modulates oxidative stress in breast cancer cell lines according to ERalpha/ERbeta ratio: Effects on mitochondrial functionality, sirtuins, uncoupling protein 2 and antioxidant enzymes. Int J Biochem Cell Biol, 2013. 45(9): p. 2045-51. 


\section{CHAPTER 5}

The mitochondrial protein mitoNEET: a study of its role in mitochondrial oxidative capacity, substrate metabolism, and lipid accumulation in skeletal muscle in rats

B.W.J. van Bree, A.R. Martins, J.A. Jörgensen, E. Kornips, G. Schaart, J. Hoeks,

P. Schrauwen

Submitted 


\section{ABSTRACT}

The mitochondrial protein mitoNEET has recently been identified as an important regulator of lipid deposition in adipose tissue by influencing mitochondrial oxidative capacity. Despite increased lipid accumulation, adipose-specific mitoNEET overexpression improved insulin sensitivity and whole-body glucose homeostasis. Since skeletal muscle also is an important tissue in glucose homeostasis, we here tested the hypotheses that 1) overexpression of mitoNEET in skeletal muscle enhances lipid storage by lowering mitochondrial oxidative capacity, and 2) mitoNEET protein expression levels are altered during the course of progressive insulin resistance.

To investigate the role of mitoNEET in skeletal muscle, mitoNEET was specifically overexpressed in tibialis anterior muscle of Wistar rats via in vivo gene electroporation. Mitochondrial oxidative capacity was evaluated in isolated mitochondria by respirometry and in tissue via ex vivo glucose and palmitate oxidation assays. The effect of mitoNEET overexpression on mitochondrial biogenesis was studied by measuring OXPHOS and PGC1 $\alpha$ protein levels. Intramyocellular lipid (IMCL) accumulation was quantified by ORO stainings. In maturating Zucker diabetic fa/fa (ZDF) rats, mitoNEET protein levels were verified by western blot.

Despite a 2.2-fold upregulation of mitoNEET protein levels in electroporated muscles, mitochondrial function, muscle substrate oxidative capacity, and IMCL levels remained unchanged. Also protein levels of OXPHOS complexes and PGC1 $\alpha$ were unaltered upon mitoNEET overexpression. Furthermore, mitoNEET expression levels were comparable between healthy $\mathrm{fa} /+$ and insulin resistant fa/fa ZDF rats, and did not change during maturation.

Our results argue against a regulating role for mitoNEET on skeletal muscle substrate oxidative capacity. Furthermore, skeletal muscle mitoNEET protein content is not associated with insulin resistance or the progression towards T2DM in ZDF rats. These data imply that mitoNEET does not play a relevant role in the regulation of glucose homeostasis. 


\section{INTRODUCTION}

Overconsumption of energy-rich food along with low physical activity leads to a chronic energy imbalance contributing to augmented lipid accumulation in adipose tissues, and eventually, development of obesity. In obesity, excessive lipid accumulation may also occur in non-adipose tissues such as skeletal muscle, which in turn is strongly associated with decreased insulin sensitivity and the development of type 2 diabetes (T2DM) [1-4]. Furthermore, obese individuals as well as T2DM patients often exhibit compromised skeletal muscle mitochondrial oxidative capacity as well as elevated markers of lipotoxicity [5-8]. These data indicate that disturbed mitochondrial oxidative capacity could be an important mediator of excessive skeletal muscle lipid accumulation within obesity and T2DM [9, 10].

MitoNEET is a protein located in the outer mitochondrial membrane (OMM) [11], which is able to influence oxidation-reduction reactions within the electron transport chain by modulating mitochondrial iron content $[12,13]$. Consequently, mitoNEET is suggested to be a powerful regulator of mitochondrial oxidative capacity $[14,15]$. It was shown that transgenic mice with adipose-specific mitoNEET overexpression had a decline in mitochondrial oxidative capacity accompanied by an enhanced lipid uptake and storage in white adipose tissue, as well as an expansion of adipose tissue [15]. Interestingly, despite this increased lipid deposition, whole-body insulin sensitivity and glucose tolerance were improved [15]. Hence, mitoNEET has been proposed to be a potential player in the progression of insulin resistance and development of T2DM [16, 17].

MitoNEET is expressed in various tissues, including skeletal muscle [14], an important tissue in glucose homeostasis accounting for $\sim 80 \%$ of insulin-stimulated glucose uptake. However, the role of mitoNEET with respect to lipid handling in skeletal muscle and its potential role in the etiology of insulin resistance and T2DM are currently unknown. In the present study we hypothesized that mitoNEET overexpression would lower muscle mitochondrial oxidative capacity, decrease muscle substrate metabolism, and increase lipid accumulation. Another goal of this study was to evaluate mitoNEET protein expression during the progression from insulin resistance towards full-blown T2DM. To address our first aim, we specifically overexpressed mitoNEET in skeletal muscle of Wistar rats through an in vivo gene electroporation technique and evaluated the effects on muscle oxidative capacity and lipid content. Furthermore, to assess whether skeletal muscle mitoNEET protein content is associated with the development of T2DM, we established its protein levels in maturating ZDF rats that are prone to develop T2DM. 


\section{MATERIALS AND METHODS}

\section{Animals}

For mitoNEET overexpression experiments, eight-week old male Wistar rats $(n=20)$ were purchased from Charles River (Maastricht, The Netherlands). In order to study mitoNEET protein levels during the progression of insulin resistance, six-week old leptin receptor deficient ZDF rats (ZDF/Gmi, fa/fa, $\mathrm{n}=30)$ and their homozygote littermates (ZDF/Gmi, +/+ (lean), $n=30$ ) were purchased from Charles River (Maastricht, The Netherlands). MitoNEET protein levels were examined in 6-, 12- and 18-week-old rats ( $\mathrm{n}=10$ per age per genotype). Data regarding the phenotype of these ZDF rats were previously reported by our group [18]. Per genotype, the animals were housed in pairs in a controlled environment $\left(21^{\circ} \mathrm{C}\right)$ with a $12 \mathrm{~h}$ light : $12 \mathrm{~h}$ dark cycle. During the entire study, Wistar rats $(\mathrm{n}=20)$ had ad libitum access to tap water and a high fat (HF) diet (45\% energy from fat, D01060502, Research Diets, New Brunswick, NJ, USA), whereas ZDF rats had ad libitum access to tap water and purina diet (Purina 5008; 16.7 energy percentage (En\%) fat, 56.4 En\% carbohydrates, and 26.8 En\% protein, Altromin Germany). Bodyweights of the rats were monitored weekly throughout the intervention period. The study complied with the principles of laboratory animal care and all protocols were approved and conducted in accordance with Maastricht University animal ethics committee guidelines.

\section{Electroporation}

Two weeks after the start of the diet, overexpression of mitoNEET in tibialis anterior (TA) muscle was accomplished by in vivo gene electroporation transfer as described previously [19]. In short, either the left or right hind limb TA of Wistar rats were used ad random to selectively overexpress mitoNEET, whereas the contralateral hind limb muscle served as a sham-electroporated internal control in which an empty vector (EV) was electroporated. For gene electroporation, rats were anesthetized with $1.5 \%$ isoflurane and $95 \% \mathrm{O}_{2}$. TA muscles were transcutaneously injected with either $150 \mu \mathrm{g}$ PCB7-MitoNEET (kindly provided by $\mathrm{P}$. Scherer) $(2 \mu \mathrm{g} / \mu \mathrm{l})$ or $150 \mu \mathrm{g}$ pcDNA3.1-EV $(2 \mu \mathrm{g} / \mu \mathrm{l})$ dissolved in sterile saline. Directly after the last injection, calibrated calipers with electrodes were placed at the ventral and dorsal side of the leg and one high voltage pulse of $800 \mathrm{~V} / \mathrm{cm}$ was applied followed by four subsequent low voltage pulses of $80 \mathrm{~V} / \mathrm{cm}$ at $1 \mathrm{~Hz}$. The electric pulses were generated by an ECM 830 electroporator (BTX, San Diego, CA, USA) as described previously [20]. Eight days after the electroporation rats were sedated by a mixture of $79 \%(\mathrm{vol} / \mathrm{vol})$ of $\mathrm{CO}_{2}$ and $21 \%(\mathrm{vol} / \mathrm{vol})$ of $\mathrm{O}_{2}$, and killed by cervical dislocation. Subsequently, TA muscles were rapidly removed and the medial piece of the muscle $(\sim 100 \mathrm{mg})$ was immediately used for either mitochondrial respirometry measurements $(n=6)$ or ex vivo substrate oxidation assays $(n=10)$. The distal 
parts of the TA were frozen in liquid nitrogen-cooled isopentane and stored at $-80^{\circ} \mathrm{C}$ until further analysis.

\section{Mitochondrial isolation}

Within 30 seconds after sacrificing the animals, both TA muscles were excised and processed on ice for mitochondrial isolation. In brief, the medial piece of the TA muscles was immediately placed into ice-cold medium containing $100 \mathrm{mM}$ sucrose, $50 \mathrm{mM} \mathrm{KCl}, 20 \mathrm{mM}$

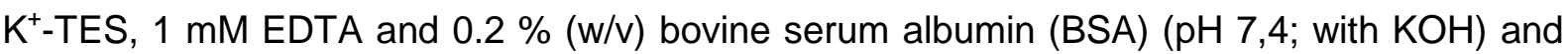
processed for mitochondrial isolation according to Nabben et al. [21]. Mitochondrial protein concentration was measured using fluorescamine (Fluram $^{\circledR}$, Fluka, Zwijndrecht, the Netherlands) with BSA as a standard [22]. Freshly isolated mitochondria were used immediately for mitochondrial respirometry. Remaining mitochondria were stored at $-80^{\circ} \mathrm{C}$ until further analysis.

\section{Mitochondrial respiration}

Respiration rates were measured in freshly isolated mitochondria from TA muscle at $37^{\circ} \mathrm{C}$ using a two-chambered Oxygraph (Oroboros Instruments, Innsbruck, Austria). Mitochondria $(0.1$ and $0.25 \mathrm{mg} / \mathrm{ml}$ mitochondrial protein for pyruvate and palmitoyl-CoA respectively) were incubated in medium consisting of $100 \mathrm{mM}$ sucrose, $50 \mathrm{mM} \mathrm{KCl}, 20 \mathrm{mM} \mathrm{K}$-TES (pH 7.2), 2 $\mathrm{mM} \mathrm{MgCl} 2,1 \mathrm{mM}$ EDTA, $4 \mathrm{mM} \mathrm{KH}_{2} \mathrm{PO}_{4}, 3 \mathrm{mM}$ malate and $0.1 \%(\mathrm{w} / \mathrm{v})$ bovine serum albumin (BSA). Pyruvate $(5 \mathrm{mM})$ was used as a carbohydrate-derived substrate and palmitoyl-CoA $(50 \mu \mathrm{M})$ with carnitine $(2 \mathrm{mM})$ as fatty acid-derived substrate. After addition of substrate maximally coupled (state 3 ) respiration was measured by adding ADP (1 mM). Subsequently, basal (state 4) respiration was estimated upon the addition of the ATP-synthase inhibitor oligomycin $(1 \mu \mathrm{g} / \mathrm{ml})$. Maximal mitochondrial respiration capacity (state $U$ respiration) was quantified by titrating the chemical uncoupler carbonyl cyanide p-trifluoromethoxyphenylhydrazone (FCCP). Mitochondrial respiration was expressed as pmol $\mathrm{O}_{2}$ per $\mathrm{mg}$ mitochondrial protein per second.

\section{Ex vivo substrate oxidation}

Ex vivo FA and glucose oxidation were assessed in homogenates of the medial parts ( 100mg) of TA muscles as previously described [23]. Briefly, TA muscles were homogenized in $1.5 \mathrm{ml}$ SET buffer (Sucrose $250 \mathrm{mM}$, Tris-HCl 10mM, ATP 2mM, EDTA $1 \mathrm{mM})$. Homogenates were plated in a modified 48-well trapping device [24]. Reactions were started by adding a combination of cold and hot substrates (for FA oxdition: palmitate $0.2 \mathrm{mM}$, $\left[1-{ }^{14} \mathrm{C}\right]$-palmitate (1 $\mu \mathrm{Ci} / \mathrm{ml}$, Perkin-Elmer, Boston, USA) $0.02 \mathrm{mM}$; for glucose oxidation: glucose $10 \mathrm{mM}$, [U- $\left.{ }^{14} \mathrm{C}\right]$-labeled glucose $(1 \mu \mathrm{Ci} / \mathrm{ml}$, Perkin-Elmer, Boston, USA $\left.)\right)$ and reaction 
media (sucrose $125 \mathrm{mM}, \mathrm{KH}_{2} \mathrm{PO}_{4} 25 \mathrm{mM}, \mathrm{KCL} 200 \mathrm{mM}$, L-carnitine $2.5 \mathrm{mM}$, malic acid $0.25 \mathrm{mM}$, Tris- $\mathrm{HCl} 20 \mathrm{mM}$, DTT 2.5mM, NAD+ 0.25mM, ATP 4mM, Coenzyme A $0.125 \mathrm{mM}$ ) to each well. Substrates were coupled to FA-free BSA. The trapping device was sealed and incubated for 2 hours at $37^{\circ} \mathrm{C}$. After incubation, all reactions were stopped by adding $70 \%$ perchloric acid and incubating the trapping device for 1 hour at room temperature. The produced ${ }^{14} \mathrm{CO}_{2}$ was trapped in the adjacent well containing $1 \mathrm{M} \mathrm{NaOH}$. Acid soluble metabolites (ASMs) were measured in supernatant of the acidified medium. ${ }^{14} \mathrm{CO}_{2}$ and $\mathrm{ASMs}$ were quantified by liquid scintillation counting. Data were normalized to protein content. Protein content in muscle homogenates was determined with a DC kit (BioRad) according to manufacturer's instructions.

\section{Western Blots}

Protein levels in TA muscle homogenates and in isolated mitochondria were determined by western blot. MitoNEET western blots were performed with muscle homogenates (4x diluted) in Bioplex buffer (BioRad, Mississauga, Ontario) mixed 1:1 with Laemmli sample buffer (BioRad, Mississauga, Ontario, Canada), or with isolated mitochondria from TA. For OXPHOS and PGC1 $\alpha$ blotting, TA muscles were homogenized in lysis buffer (10\% NP40, $10 \%$ SDS, 100mM PMSF in PBS) supplemented with a phosphatase and protease inhibitor cocktail (Roche). Western blot analyses were then performed as described before [19]. In short, standard SDS-PAGE, blocking and incubation protocols were followed. Blots were incubated overnight at room temperature with primary antibodies directed against mitoNEET

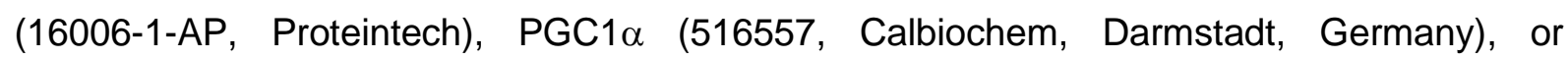
structural components of the electron transport chain and oxidative phosphorylation (rodent OXPHOS antibody cocktail, MS604; MitoSciences, Eugene, OR, USA). After the primary incubation period, appropriate secondary antibodies (IRDye800- or IRDye700-conjugated secondary antibodies, Rockland, Tebu-bio, Heerhugowaard, The Netherlands) were applied and blots were visualized using the Odyssey Near Infrared Imager (Licor Biosciences, Westburg, Leusden, The Netherlands). Blots were quantified using Quantity One software (version 4.6.9; Biorad) and protein levels were expressed as arbitrary units (AU).

Overexpression of mitoNEET was considered successful when mitoNEET protein levels in muscle lysates of the mitoNEET electroporated leg were at least $30 \%$ higher in comparison to the empty vector electroporated TA muscle.

\section{MitoNEET protein levels in ZDF rats}

MitoNEET protein levels were determined in TA muscle homogenates of fa/fa ZDF rats and their homozygote littermates (+/+ ZDF rats) at the age of 6, 12 and 18 weeks [18]. MitoNEET 
protein levels were quantified by western blots using the primary antibody directed against mitoNEET (16006-1-AP, Proteintech).

\section{Oil-red-O staining and immunofluorescence}

To quantify the neutral lipid storage in skeletal muscle, cryosections $(5 \mu \mathrm{m})$ of TA muscles were stained with oil red $\mathrm{O}(\mathrm{ORO})$ as previously described [25]. ORO staining was combined with laminin staining (L-9393, Sigma, St Louis, USA) to visualize the cell membranes and with MitoNEET staining (16006-1-AP, Proteintech). Sections were examined using a Nikon E800 fluorescence microscope (Uvikon, Bunnik, The Netherlands). Digital images were captured and processed using Lucia G/F 5.49 image analysis software (Nikon, Dusseldorf, Germany). IMCL content was calculated as percentage per cell surface area.

\section{Statistical analysis}

Results are presented as mean \pm SEM. Effects of mitoNEET overexpression were evaluated with paired T-tests (two-tailed). MitoNEET protein content in ZDF rats was analyzed with a two-tailed unpaired T-test. Outcomes were considered significant when $\mathrm{P}<0.05$. All statistical analyses were performed using GraphPad Prism 5.0a Macintosh Version.

\section{RESULTS}

\section{Body weight}

All Wistar rats were provided a high fat (HF) diet for a period of 3 weeks. At the start of the dietary intervention, body weight averaged $260 \pm 4 \mathrm{~g}$ and was significantly increased after 2 weeks of HF diet $(330 \pm 3 g, p=0.003)$, i.e. at the time of electroporation and at the end of the study, 8 days after electroporation $(360 \pm 3 g, p<0.0001)$. Body weight data from the ZDF rats were reported previously [18].

\section{MitoNEET overexpression}

The in vivo gene electroporation procedure was used to selectively and unilaterally overexpress mitoNEET in TA muscle while the contralateral TA muscle served as a shamelectroporated internal control. In the whole group $(n=20)$, we found a significant $(P<0.0001)$ 1.8-fold upregulation of mitoNEET protein expression in TA muscle $(1.0 \pm 0.1 \mathrm{AU}$ in EV vs. $1.79 \pm 0.2 \mathrm{AU}$ in mitoNEET electroporated TA). Only the rats with $\geq 30 \%$ mitoNEET overexpression were included for follow-up analyses. As a result, 4 rats were excluded and the remaining 16 rats displayed a $\sim 2.2$-fold overexpression of the mitoNEET protein in TA muscle 8 days after the electroporation procedure $(P<0.0001$, figure 1$)$. 


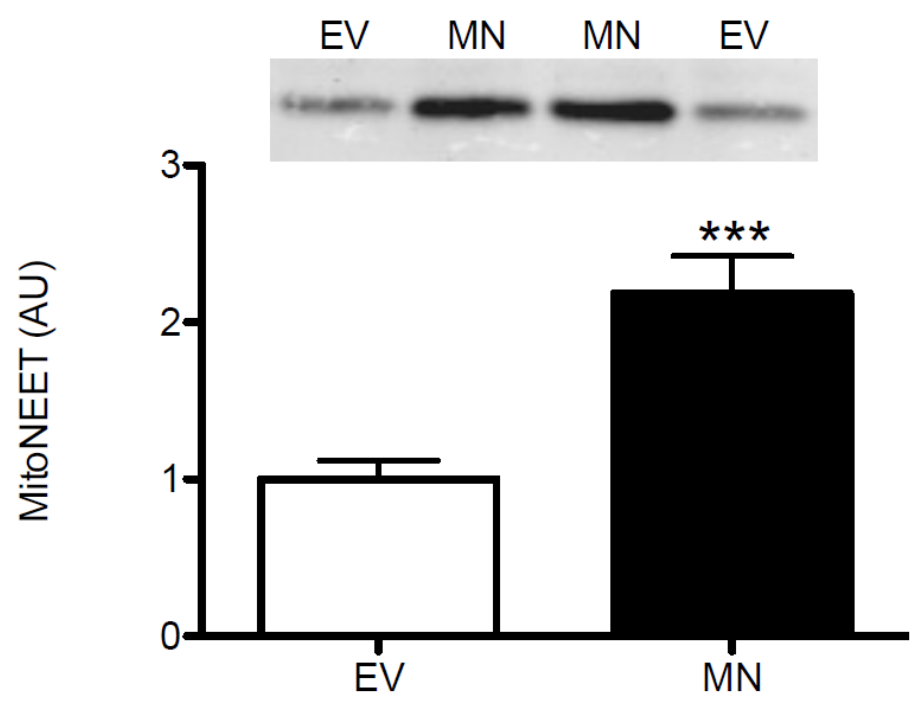

Figure 1. MitoNEET (MN) overexpression was accomplished in tibialis anterior (TA) muscle of Wistar rats $(n=16)$ by in vivo gene electroporation. MN protein levels (AU), as quantified by western blot analysis, were significantly upregulated in the $M N$ in comparison to the empty vector (EV) electroporated muscle. MN protein content in EV muscle was set to 1.0. ${ }^{* * *}$ : $\mathrm{P}<0.0001$.

\section{Unaffected skeletal muscle metabolism upon mitoNEET overexpression}

In a subset of rats $(n=6)$, we established mitochondrial respiratory capacity by performing oxygen consumption measurements in isolated mitochondria (figure 2). Both ADP-driven (state 3 ) and maximally uncoupled (FCCP-stimulated, state $U$ ) respiration on both pyruvate $(\mathrm{P}=0.34$ and $\mathrm{P}=0.41$ for states 3 and $U$ respectively) (figure $2 \mathrm{~A}-\mathrm{B}$ ) and palmitoyl-CoA + carnitine $(P=0.65$ and $P=0.54$ for states 3 and $U$ respectively) (figure $2 C-D$ ) remained unaffected upon mitoNEET overexpression.
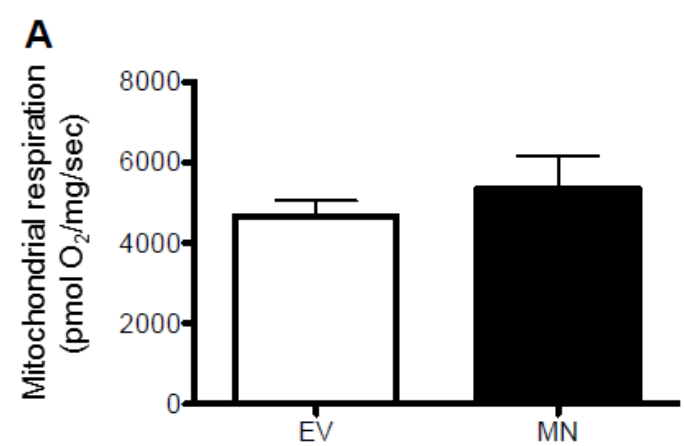

State 3

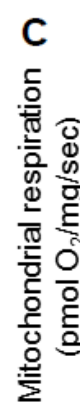

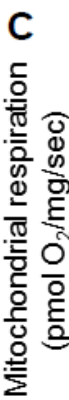

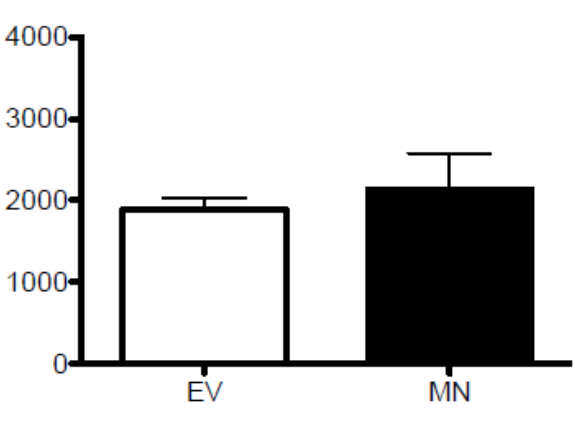

State 3
B

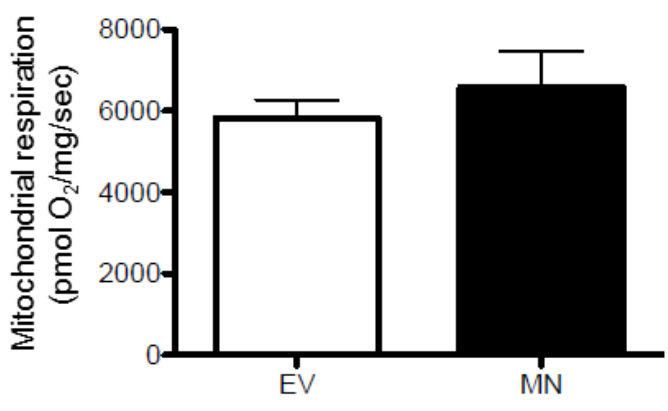

State U

D

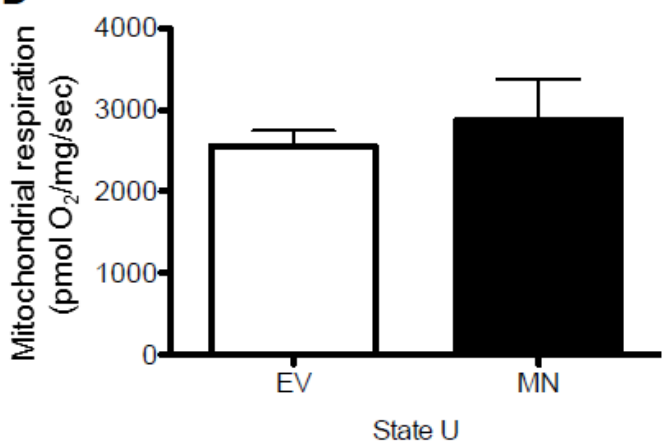

Figure 2. Oxidative capacity of isolated mitochondria from TA muscle is not affected upon MN overexpression in rats $(n=6)$. (A) Coupled (ADP-driven) mitochondrial state 3 respiration upon pyruvate, (B) Uncoupled (FCCPstimulated) state $U$ respiration on pyruvate. (C) State 3 on palmitoyl-CoA and (D) State $U$ on palmitoyl-CoA in mitochondria isolated from TA muscles electroporated with either EV or MN. 
We also performed ex vivo glucose and palmitate oxidation assays in TA muscle homogenates from another subgroup of rats $(n=10)$. MitoNEET overexpression did not alter the complete oxidation of either glucose (figure 3 ) or palmitate (figure 4$)$ to $\mathrm{CO}_{2}(\mathrm{P}=0.21$ and $\mathrm{P}=0.40$ for glucose and palmitate, respectively). Also acid soluble metabolites (ASMs) were comparable between mitoNEET overexpressing muscle homogenates and the EV controls, regardless of the substrate $(P=0.09$ and $P=0.43$ for glucose and palmitate respectively, figures 3 and 4). Interestingly, neutral lipid incorporation tended to be reduced in mitoNEET overexpressing samples ( $P=0.07$, figure 4$)$.

A

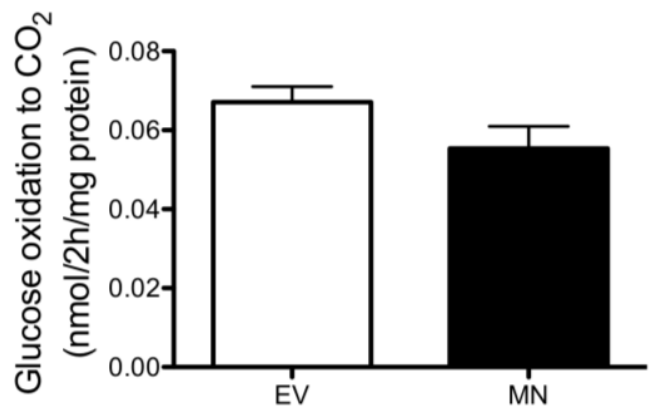

B

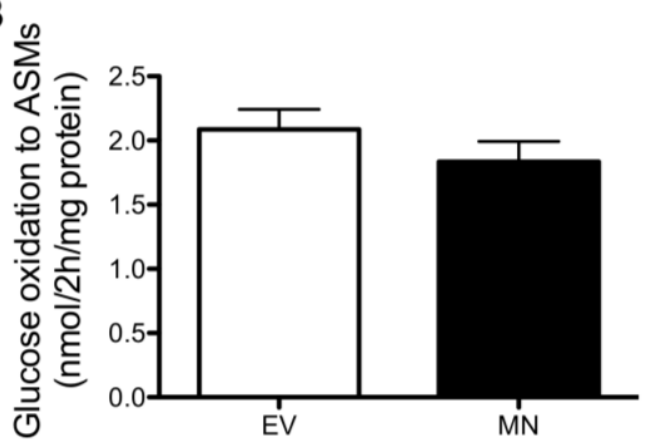

Figure 3. Ex vivo glucose oxidation to $\mathrm{CO}_{2}$ (A) and to acid soluble metabolites (ASMs) (B) was not influenced by $\mathrm{MN}$ overexpression in TA muscle homogenates $(\mathrm{n}=10)$.

A

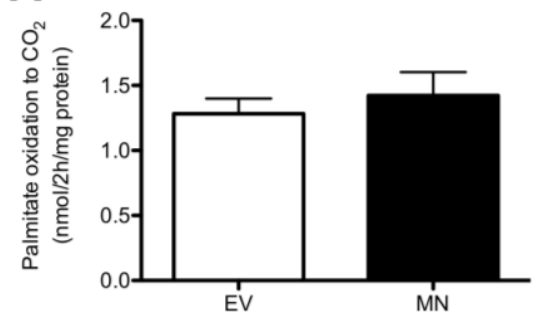

B

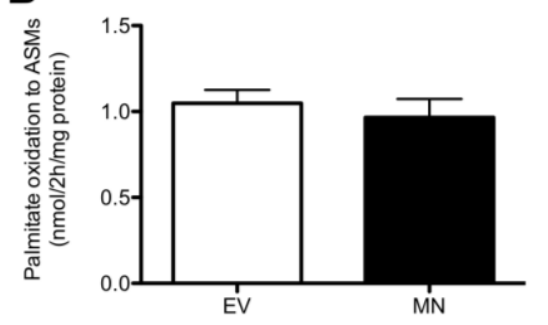

C

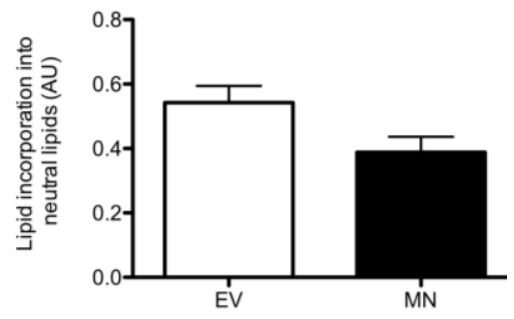

Figure 4. Palmitate oxidation to $\mathrm{CO}_{2}$ (A) and acid soluble metabolites (ASMs) (B) was not influenced by $\mathrm{MN}$ overexpression in TA muscle homogenates $(n=10)$. (C) Lipid incorporation into neutral lipids tended to be decreased upon $\mathrm{MN}$ overexpression $(\mathrm{P}=0.07)$.

\section{Mitochondrial density and biogenesis remained unchanged upon mitoNEET overexpression}

Next, we measured the effect of mitoNEET overexpression on markers for mitochondrial density and -biogenesis. Quantification of western blots revealed no effect of mitoNEET overexpression on the distinct measured structural components of the electron transport chain and oxidative phosphorylation (OXPHOS complexes I, II, III and V) (figure 5). Additionally, elevated mitoNEET protein levels were not paralleled by changes in PGC1 $\alpha$ protein content (figure 5). 


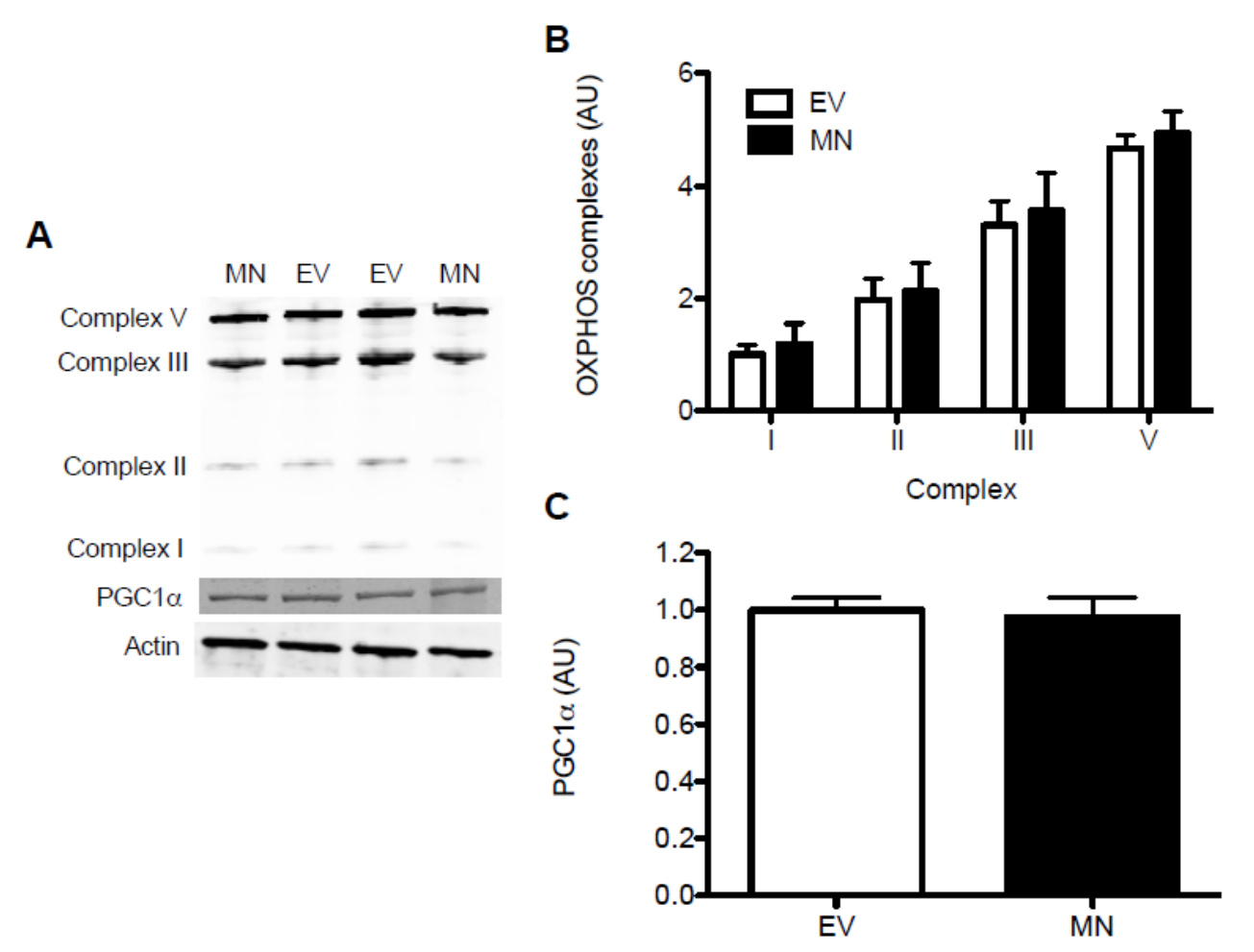

Figure 5. Structural components of the electron transport chain and oxidative phosphorylation (OXPHOS) and PGC1 $\alpha$ were measured to estimate the effect of MN overexpression on mitochondrial density and biogenesis in tibialis anterior (TA) muscle $(n=6)$. (A) Protein levels of OXPHOS complexes and PGC1 $\alpha$ were quantified by western blot analysis. (B) In vivo MN electroporation did not result in differences in any of the OXPHOS complexes. Protein expression levels of complex I in EV electroporated muscle was set to $1.0 \mathrm{AU}$. (C) PGC1 $\alpha$ was also unaffected by MN overexpression. PGC1 $\alpha$ protein content in EV electroporated TA was set to $1.0 \mathrm{AU}$.

\section{MitoNEET protein levels are not associated with IMCL content}

MitoNEET has been shown to increase lipid uptake and storage in adipose tissue and liver [15]. To examine whether mitoNEET has similar effects in skeletal muscle we quantified IMCL levels by ORO stainings. However, overexpression of mitoNEET in TA did not significantly alter IMCL content $(\mathrm{P}=0.78$, figure 6$)$ as was reflected by similar percentages IMCL per cell surface area in EV and mitoNEET electroporated muscles: $0.96 \pm 0.29 \%$ vs. $0.81 \pm 0.32 \%$ respectively.

\section{MitoNEET protein content under conditions of insulin resistance}

To explore whether mitoNEET is a potential contributor to enhanced IMCL levels at different stages in the development of T2DM, we also measured mitoNEET protein levels in muscle of maturating ZDF rats, i.e. at 6,12 , and 18 weeks of age. Protein levels of mitoNEET were comparable between the insulin resistant fa/fa ZDF rats and their insulin sensitive littermates $(\mathrm{fa} /+)$ at all 3 ages tested $(1.0 \pm 0.1 \mathrm{AU}$ vs. $1.13 \pm 0.18 \mathrm{AU}, 1.30 \pm 0.13 \mathrm{AU}$ vs. $1.33 \pm 0.12 \mathrm{AU}$, $1.32 \pm 0.17 \mathrm{AU}$ vs. $1.27 \pm 0.12 \mathrm{AU}$ for $\mathrm{fa} /+\mathrm{vs}$. fa/fa rats at 6,12 and 18 weeks of age respectively) (figure 7 ). 
A

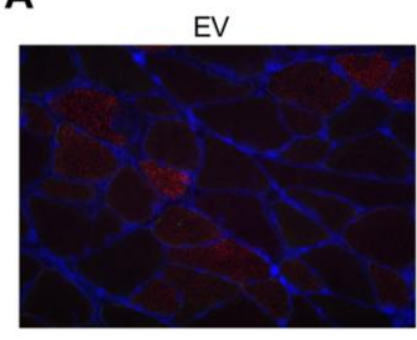

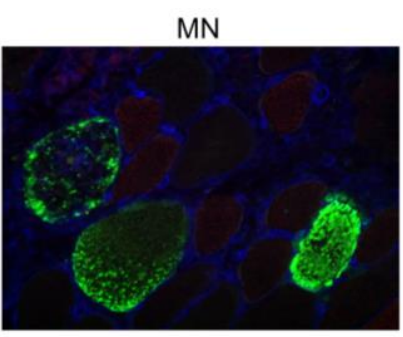

B

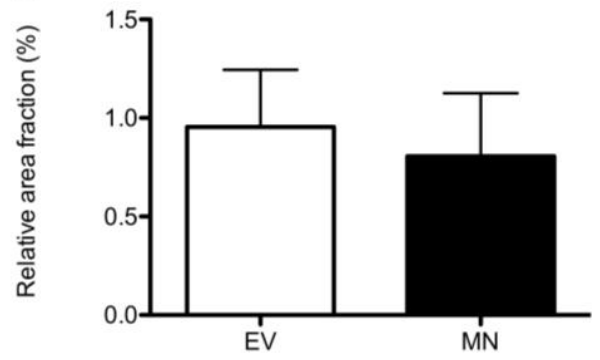

Figure 6. Overexpression of the mitochondrial protein MN does not influence IMCL accumulation in tibialis anterior muscle $(n=6)$. IMCL was quantified by an Oil Red O (ORO) staining. (A) Representative microscope images of ORO staining (red) combined with laminin (blue) and MN (green) in TA muscle sections electroporated either with EV or MN. (B) ORO quantification expressed as percentage relative area fraction. IMCL levels were similar in EV and MN electroporated muscles.

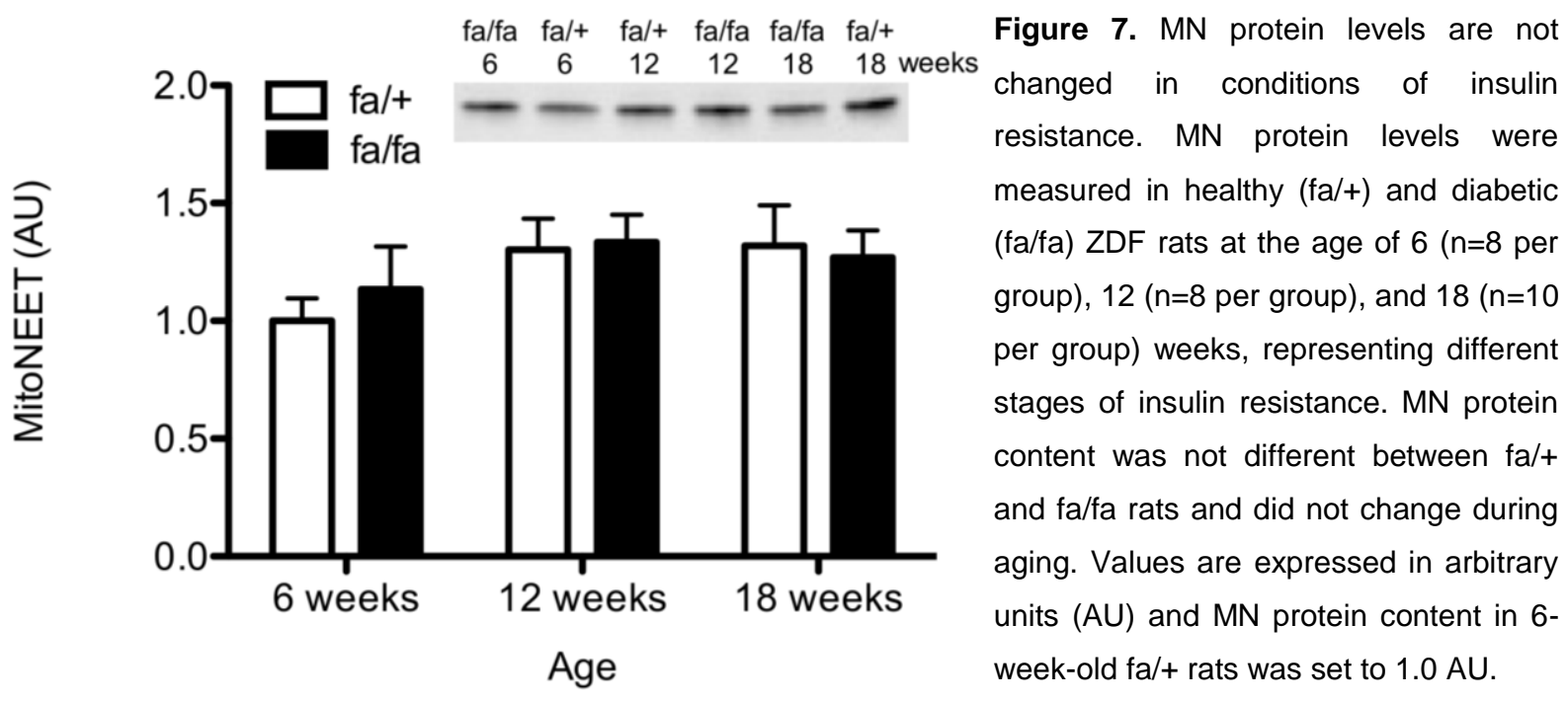

\section{DISCUSSION}

Recently, the mitochondrial protein mitoNEET has been identified as a potent regulator of mitochondrial oxidative capacity, and therefore, may impact substrate metabolism. Because disturbed mitochondrial function and lipid metabolism in muscle are related with the development of T2DM, we here studied the effect of mitoNEET overexpression in skeletal muscle. We show that skeletal muscle mitochondrial capacity, substrate oxidation and lipid accumulation were not affected by mitoNEET overexpression. Furthermore, Zucker Diabetic Fatty (ZDF) rats displayed similar mitoNEET protein levels in skeletal muscle during maturation when compared with lean, healthy littermates.

MitoNEET is a protein located in the outer mitochondrial membrane (OMM) [11] and belongs to a small family of $2 \mathrm{Fe}-2 \mathrm{~S}$ proteins that are characterized by a well-conserved CDGSH domain [26]. The CDGSH domain of mitoNEET is oriented toward the cytoplasm 
and is involved in binding of iron [12, 13]. MitoNEET has been postulated to inhibit the electron transport chain (ETC) and oxidative capacity, via inhibition of mitochondrial iron transport [14, 15, 27], as iron is a rate-limiting component for ETC activity [28]. In this perspective, a 2-fold reduction of mitochondrial iron content was reported in mitochondria isolated from adipose-specific mitoNEET overexpressing mice, which was complemented by lowered ( 30\%) mitochondrial oxygen consumption rates [15]. To investigate the role of mitoNEET in skeletal muscle, we specifically overexpressed mitoNEET in the tibialis anterior by in vivo gene electroporation. Our approach was proven successful as mitoNEET protein levels were more than doubled in tibialis anterior muscles. Next, we anticipated that the increased expression of mitoNEET would be paralleled by lowered mitochondrial oxidative capacity in skeletal muscle. To address our hypothesis, we performed oxygen consumption measurements in isolated skeletal muscle mitochondria, using both carbohydrate- and fatty acid-derived substrates. Surprisingly, we found no effect of mitoNEET overexpression on the mitochondrial capacity to oxidize either glucose or fatty acids. Because mitoNEET is located in the outer mitochondrial membrane with the iron-binding domain facing the cytoplasm, one could argue that mitoNEET protein was lost or inactivated during the mitochondrial isolation procedure, especially since we used a protease during the isolation. To exclude the possibility that our data on mitochondrial respiration were influenced by the mitochondrial isolation procedure, we also measured ex vivo skeletal muscle substrate oxidation capacity in whole TA muscle homogenates, thereby excluding the protease treatment. However, using this method we also did not observe any changes in the capacity to oxidize either glucose or palmitate within skeletal muscle. Taken together, our data imply that mitoNEET does not significantly influence the capacity of muscle to oxidize fatty acids or carbohydrates.

Our results differ from findings in adipose tissue and liver, where overexpression of mitoNEET resulted in a lowered mitochondrial oxidative capacity whereas a reduction in mitoNEET expression lead to enhanced mitochondrial respiration [15]. The discrepancy in these results may indicate tissue-specificity in the physiological role of mitoNEET. This notion is supported by the observation that complex I-driven state 3 respiration and maximal uncoupled respiration rates were shown to be decreased in mitochondria isolated from hearts of mitoNEET-null mice [14].

Besides its direct effect on mitochondrial iron metabolism and ETC activity, mitoNEET has also been proposed to stimulate mitochondrial biogenesis via PGC1 $\alpha$ induced pathways $[15,27]$. In adipose tissue, PGC1 $\alpha$ gene expression was markedly elevated in response to mitoNEET overexpression [15]. Furthermore, mitoNEET overexpressing epithelial breast cancer (MDA-MB-231) cells also displayed elevated PGC1 $\alpha$ expression, which was paralleled by similar increases in the protein levels of OXPHOS complexes [27]. In the present study, protein levels of PGC1 $\alpha$ and OXPHOS complexes remained unchanged upon 
overexpression of mitoNEET in skeletal muscle, indicative of an unaltered mitochondrial content. These data are in line with the lack of effect on muscle oxidative capacity, observed in isolated mitochondria and whole-muscle homogenates.

Previously, it was shown that adipose-specific mitoNEET overexpression in mice resulted into an enhancement in lipid uptake, storage, and eventually expansion of adipose tissue and amount of lipid droplets [14, 15]. Noteworthy, liver fat content in these adiposespecific transgenic mice decreased and liver insulin sensitivity was enhanced as was demonstrated by a decrease in hepatic lipid content and glucose output [15], which could also impact the described whole-body metabolic improvements. These findings prompted us to examine the effect of mitoNEET overexpression in skeletal muscle on IMCL content. Consistent with our data on (fat) oxidative capacity however, IMCL levels remained unchanged upon mitoNEET overexpression. Therefore, our data do not support a key role for mitoNEET in skeletal muscle oxidative capacity and lipid handling.

Finally, we explored whether skeletal muscle protein expression of mitoNEET was affected during the transition of a pre-diabetic state towards full-blown T2DM. For this purpose, mitoNEET protein content was quantified in skeletal muscle of ZDF rats during maturation, at the age of 6,12 , and 18 weeks. During maturation the metabolic profile of ZDF rats progresses from a pre-diabetic state (at 6 weeks of age) towards an advanced hyperglycemic hyperinsulinemic state (at 18 weeks of age) [29]. In these animals, we have previously shown that progression of insulin resistance during maturation of occurs in parallel with increased IMCL accumulation [18, 29]. Because mitoNEET has been shown to enhance lipid uptake and storage in adipose tissue and liver [15], we here tested whether mitoNEET could be a potential contributor to the enhanced IMCL levels during progression of insulin resistance in ZDF rats. Nevertheless, since we found similar levels of mitoNEET protein expression in skeletal muscle of diabetic vs. healthy ZDF rats at either 6, 12, and 18 weeks of age, our results disconnect skeletal muscle mitoNEET protein levels from the development of T2DM and from IMCL accumulation. It has been described that mitochondrial dysfunction is not a prerequisite for the development of T2DM per se in these ZDF rats, as fat oxidative capacity is reduced in pre-diabetic rats at 6 weeks of age, which was compensated for by improved mitochondrial capacity in 12-week-old rats despite developing hyperglycemia in this period [18]. However, increased IMCL content seems to be an important determinant in the etiology of insulin resistance in diabetes prone ZDF rats [18, 29]. Therefore, the data obtained in this study imply that mitoNEET does not significantly contribute to skeletal muscle insulin sensitivity. To the best of our knowledge, this is the first study examining mitoNEET protein expression levels within skeletal muscle in a model of progressive insulin resistance and T2DM. Nevertheless, in white adipose tissue and liver mitoNEET has been shown to improve glucose homeostasis after exposure to a HF diet, as both insulin sensitivity 
and glucose tolerance increased by mitoNEET overexpression in these tissues [15]. Collectively, these and our data suggest that mitoNEET could influence the progression of insulin resistance and development of T2DM by affecting glucose homeostasis in white adipose tissue and liver, but not skeletal muscle.

In summary, skeletal muscle specific overexpression of the mitochondrial protein mitoNEET did not result in changes in mitochondrial oxidative capacity and -density, wholemuscle substrate metabolism, or lipid content. Also, no indications were found for a relevant physiological role in skeletal muscle of mitoNEET in the transition from pre-diabetes toward advanced T2DM. Therefore, our data argue against an important role for mitoNEET in glucose homeostasis in skeletal muscle.

\section{Acknowledgements}

The authors would like to thank P. Scherer for donating the mitoNEET construct.

\section{REFERENCES}

1. Savage, D.B., K.F. Petersen, and G.I. Shulman, Disordered lipid metabolism and the pathogenesis of insulin resistance. Physiol Rev, 2007. 87(2): p. 507-20.

2. Chow, L., A. From, and E. Seaquist, Skeletal muscle insulin resistance: the interplay of local lipid excess and mitochondrial dysfunction. Metabolism, 2010. 59(1): p. 70-85.

3. Holloway, G.P., A. Bonen, and L.L. Spriet, Regulation of skeletal muscle mitochondrial fatty acid metabolism in lean and obese individuals. Am J Clin Nutr, 2009. 89(1): p. 455S-62S.

4. Goodpaster, B.H., et al., Intramuscular lipid content is increased in obesity and decreased by weight loss. Metabolism, 2000. 49(4): p. 467-72.

5. Kelley, D.E., et al., Dysfunction of mitochondria in human skeletal muscle in type 2 diabetes. Diabetes, 2002. 51(10): p. 2944-50.

6. Ritov, V.B., et al., Deficiency of subsarcolemmal mitochondria in obesity and type 2 diabetes. Diabetes, 2005. 54(1): p. 8-14.

7. Perseghin, G., et al., Intramyocellular triglyceride content is a determinant of in vivo insulin resistance in humans: a $1 \mathrm{H}-13 \mathrm{C}$ nuclear magnetic resonance spectroscopy assessment in offspring of type 2 diabetic parents. Diabetes, 1999. 48(8): p. 1600-6.

8. Schaffer, J.E., Lipotoxicity: when tissues overeat. Curr Opin Lipidol, 2003. 14(3): p. 281-7.

9. Petersen, K.F., et al., Impaired mitochondrial activity in the insulin-resistant offspring of patients with type 2 diabetes. N Engl J Med, 2004. 350(7): p. 664-71.

10. Morino, K., K.F. Petersen, and G.I. Shulman, Molecular mechanisms of insulin resistance in humans and their potential links with mitochondrial dysfunction. Diabetes, 2006. 55 Suppl 2: p. S9-S15.

11. Paddock, M.L., et al., MitoNEET is a uniquely folded 2Fe $2 S$ outer mitochondrial membrane protein stabilized by pioglitazone. Proc Natl Acad Sci U S A, 2007. 104(36): p. $14342-7$.

12. Lin, J., et al., Crystal structure of human mitoNEET reveals distinct groups of iron sulfur proteins. Proc Natl Acad Sci U S A, 2007. 104(37): p. 14640-5.

13. Wiley, S.E., et al., The outer mitochondrial membrane protein mitoNEET contains a novel redox-active 2Fe-2S cluster. J Biol Chem, 2007. 282(33): p. 23745-9.

14. Wiley, S.E., et al., MitoNEET is an iron-containing outer mitochondrial membrane protein that regulates oxidative capacity. Proc Natl Acad Sci U S A, 2007. 104(13): p. 5318-23. 
15. Kusminski, C.M., et al., MitoNEET-driven alterations in adipocyte mitochondrial activity reveal a crucial adaptive process that preserves insulin sensitivity in obesity. Nat Med, 2012. 18(10): p. 1539-49.

16. Zuris, J.A., et al., Facile transfer of [2Fe-2S] clusters from the diabetes drug target mitoNEET to an apo-acceptor protein. Proc Natl Acad Sci U S A, 2011. 108(32): p. 13047-52.

17. Zuris, J.A., et al., NADPH inhibits [2Fe-2S] cluster protein transfer from diabetes drug target MitoNEET to an apo-acceptor protein. J Biol Chem, 2012. 287(15): p. 11649-55.

18. Lenaers, E., et al., Adaptations in mitochondrial function parallel, but fail to rescue, the transition to severe hyperglycemia and hyperinsulinemia: a study in Zucker diabetic fatty rats. Obesity (Silver Spring, Md.), 2010. 18(6): p. 1100-1107.

19. Bosma, M., et al., Overexpression of PLIN5 in skeletal muscle promotes oxidative gene expression and intramyocellular lipid content without compromising insulin sensitivity. Biochim Biophys Acta, 2013. 1831(4): p. 844-52.

20. Bruce, C.R., et al., Overexpression of carnitine palmitoyltransferase-1 in skeletal muscle is sufficient to enhance fatty acid oxidation and improve high-fat diet-induced insulin resistance. Diabetes, 2009. 58(3): p. 550-8.

21. Nabben, M., et al., Uncoupled respiration, ROS production, acute lipotoxicity and oxidative damage in isolated skeletal muscle mitochondria from UCP3-ablated mice. Biochim Biophys Acta, 2011. 1807(9): p. 1095-105.

22. Schrauwen, P., et al., Uncoupling protein 3 as a mitochondrial fatty acid anion exporter. FASEB J, 2003. 17(15): p. 2272-4.

23. Hulver, M.W., et al., Skeletal muscle lipid metabolism with obesity. Am J Physiol Endocrinol Metab, 2003. 284(4): p. E741-7.

24. Ukropcova, B., et al., Dynamic changes in fat oxidation in human primary myocytes mirror metabolic characteristics of the donor. J Clin Invest, 2005. 115(7): p. 1934-41.

25. Koopman, R., G. Schaart, and M.K. Hesselink, Optimisation of oil red O staining permits combination with immunofluorescence and automated quantification of lipids. Histochem Cell Biol, 2001. 116(1): p. 63-8.

26. Lin, J., et al., Structure and molecular evolution of CDGSH iron-sulfur domains. PLoS One, 2011. 6(9): p. e24790.

27. Salem, A.F., et al., Mitochondrial biogenesis in epithelial cancer cells promotes breast cancer tumor growth and confers autophagy resistance. Cell Cycle, 2012. 11(22): p. 4174-80.

28. Macdonald, V.W., S. Charache, and P.J. Hathaway, Iron deficiency anemia: mitochondrial alpha-glycerophosphate dehydrogenase in guinea pig skeletal muscle. $\mathrm{J}$ Lab Clin Med, 1985. 105(1): p. 11-8.

29. De Feyter, H.M., et al., Increased intramyocellular lipid content but normal skeletal muscle mitochondrial oxidative capacity throughout the pathogenesis of type 2 diabetes. FASEB J, 2008. 22(11): p. 3947-55. 


\section{CHAPTER 6}

\section{Lack of UCP3 does not affect skeletal muscle mitochondrial function under lipid-challenged conditions, but leads to sudden cardiac death}

Bianca W.J. van Bree*, Miranda Nabben*, Ellen Lenaers, Joris Hoeks, Matthijs K. C. Hesselink, Gert Schaart, Marion J.J. Gijbels, Jan F.C. Glatz, Gustavo J.J. da Silva, Leon J. de Windt, Rong Tian, Elise Mike, Darlene G. Skapura, Xander H.T. Wehrens, and Patrick

Schrauwen

Submitted 


\section{ABSTRACT}

Skeletal muscle (skm) uncoupling protein 3 (UCP3) levels are strongly reduced in type 2 diabetics, who often exhibit mitochondrial dysfunction and lipotoxicity. We hypothesized a role for UCP3 in preservation of mitochondrial function under lipid-challenged conditions. Contradictory, elevated cardiac UCP3 is suggested to reduce cardiac efficiency and oxidative capacity, especially upon lipid overload. Hence, UCP3s exact physiological function in lipid handling in skeletal and cardiac muscle remains unknown. Interestingly, etomoxir, a fat oxidation inhibitor and strong inducer of UCP3, is proposed for treating both diabetes and heart failure. To evaluate UCP3s role in skm and heart under lipid-challenged conditions, the effect of UCP3 ablation was examined in a state of dysbalance between fat availability and oxidative capacity.

Wild type (WT) and UCP3 ${ }^{--}$mice were subjected to high-fat feeding combined with etomoxir. UCP3 did not seem to affect mitochondrial lipotoxicity in skm. However, we observed $25 \%$ mortality in $\mathrm{UCP}^{-/}$s upon etomoxir-administration vs. $11 \%$ in WTs. Analyses of cardiac tissue revealed no major role for UCP3 in preventing cardiac lipotoxicity, apoptosis, fibrosis (histology, immunohistochemistry), oxidative capacity (respirometry) or function (echocardiography). Electrophysiology demonstrated however, prolonged QRS and QTc intervals and greater susceptibility to ventricular tachycardia upon programmed electrical stimulation in $\mathrm{UCP}^{-/} \mathrm{s}$ upon etomoxir. Isoproterenol administration after pacing resulted in $75 \%$ mortality in UCP3 ${ }^{-/-}$mice vs. $14 \%$ in WT.

Our results argue against a protective role for UCP3 on skm metabolism under lipid overload, but suggest UCP3 to be crucial in prevention of arrhythmias upon lipid -challenged conditions. 


\section{INTRODUCTION}

The inner mitochondrial membrane uncoupling proteins (UCPs) are suggested to regulate the proton gradient over the inner mitochondrial membrane, thereby regulating mitochondrial ATP production and/or mitochondrial reactive oxygen species production [1, 2]. Despite its homology to the well-characterized brown-adipose tissue specific UCP1, the exact mechanism and physiological function for the skeletal and cardiac muscle variant UCP3 has not been revealed. UCP3 has been closely tied to fatty acid (FA) metabolism, as UCP3 expression is downregulated when FA oxidation is improved, such as following endurance training [3, 4] or (pharmacologically-induced) weight reduction [5]. Conversely, UPC3 levels are upregulated when the FA supply exceeds oxidation, e.g., upon a high-fat diet [6], lipid infusion [7], fasting [8], or upon pharmacological inhibition of fat oxidation by etomoxir [9, 10].

UCP3 is mainly expressed in skeletal and cardiac muscle. It has been shown that UCP3 is reduced by $50 \%$ in skeletal muscle (skm) of obese type 2 diabetes patients [11, 12], who often exhibit mitochondrial dysfunction, lipotoxicity and elevated oxidative stress [2, 13]. In contrast UCP3 expression is markedly increased in cardiac tissue of human heart failure patients [14]. Using transgenic animal models, it was recently shown that UCP3 might affect cardiac energy efficiency under high-fat conditions [15], suggesting that elevated UCP3 levels result in energy deficiency that is characteristic for heart failure. However, the potential role of UCP3 in skeletal and cardiac muscle is still under debate.

One of the strongest inductions of UCP3 can be achieved by the pharmacological inhibition of fat oxidation with etomoxir, both in humans and rodents $[9,10]$. This compound is able to reduce the mitochondrial entry of long-chain fatty acids via irreversible inhibition of carnitine palmitoyl transferase-1 (CPT-1), thereby inhibiting mitochondrial lipid oxidation and forcing increased glucose oxidation [16]. Etomoxir, or CPT1 inhibition in general, has been proposed as a therapeutic target for the treatment of heart failure [17, 18], since a shift towards glucose oxidation may improve cardiac energy efficiency [16]. In addition, inhibiting fatty acid oxidation to force glucose oxidation has been suggested to improve skm insulin resistance $[17,19]$. We have previously hypothesized that the strong induction of UCP3 upon the inhibition of fat oxidation serves to prevent mitochondrial lipotoxicity, however experimental data so far is lacking.

In order to clarify the role of UCP3 during lipid-challenged conditions, we investigated the effect of etomoxir administration on skeletal and cardiac muscle energy and lipid metabolism in $\mathrm{UCP}^{-{ }^{-}}$mice and WT littermates. Our data suggest that there was no significant role for skm UCP3 in protection against lipid-induced mitochondrial dysfunction. However, cardiac UCP3 was crucial to maintain cardiac function, and absence of UCP3 under lipid-challenging conditions markedly enhanced the risk of sudden cardiac death. 


\section{MATERIALS AND METHODS}

\section{Animals}

Male UCP3 knockout mice (UCP3 ${ }^{-/}$) and wild type (WT) C57BI/6 control mice ( $\mathrm{n}=7-8$ per group, unless otherwise stated, age 14-15 weeks) received a high-fat diet (HF-diet; $45 \%$ of energy as fat, D01060502, Research Diets, New Brunswick, USA) for a period of 14 days. Animals were housed individually in a controlled environment $\left(21-22^{\circ} \mathrm{C}\right)$ on a $12 \mathrm{~h} \mathrm{light/dark}$ cycle (light from 07:00 to 19:00h) and had free access to food and tap water. At day 6 of the dietary intervention both mice strains were randomly divided into two groups: (1) etomoxir, or (2) saline. During the remaining 8 days of the dietary intervention, animals received a daily dose (at the beginning of the light cycle, at 07:00h) of either etomoxir (experimental groups; $((+)$-etomoxir sodium salt hydrate $20 \mathrm{mg} / \mathrm{kg}$ body weight, dissolved in $0.9 \% \mathrm{NaCl}(\mathrm{w} / \mathrm{v})$ (as previously used by Luiken et al. [20]); Sigma Aldrich, St. Louis, MO, USA) or saline (control groups; $0.9 \% \mathrm{NaCl}$ ) via intraperitoneal (i.p.) injections. The last injection was administered 24 hours before sacrificing the animals. All experiments were approved by the Institutional Animal Care and Use Committee of Maastricht University and Baylor College of Medicine and complied with the principles of laboratory animal care.

\section{Tissue collection}

Animals were sedated using a mixture of $79 \% \mathrm{CO}_{2}$ and $21 \% \mathrm{O}_{2}$ and decapitated. Skeletal muscle from both hind limbs ( 2.0 g) was rapidly dissected and placed immediately into icecold mitochondrial isolation medium containing $100 \mathrm{mM}$ sucrose, $50 \mathrm{mM} \mathrm{KCL}, 20 \mathrm{mM} \mathrm{K+-}$ TES, $1 \mathrm{mM}$ EDTA, and $0.2 \%(\mathrm{w} / \mathrm{v})$ fatty acid free bovine serum albumin (BSA). Both tibialis anterior muscles, as well as hearts, were isolated and frozen into liquid nitrogen and stored at $-80^{\circ} \mathrm{C}$ until further protein, immunohistochemical, and enzymatic analysis.

In a subset of mice, the heart was rapidly dissected and treated under similar conditions. Skeletal muscle from these mice was frozen and stored at $-80^{\circ} \mathrm{C}$ until further analysis.

\section{Protein analyses}

Western blotting technique was used for protein analysis of mouse UCP3, Oxphos (as a measure for mitochondrial density), adenine nucleotide transporter (ANT; a protein suggested to be involved in mitochondrial uncoupling) and 4-hydroxynonenal (4-HNE; as a marker for lipid peroxidation) as previously described [21]. Equal amounts of protein, as determined by Coomassie Briliant Blue staining, were loaded onto a polyacrylamide gel and western blotting was performed using rabbit polyclonal antibody for determination of mouse UCP3 (code 1338, kindly provided by L.J. Slieker, Eli Lilly) [22], a mixture of monoclonal 
antibodies specific for structural components of the oxidative phosphorylation (Oxphos) complexes (MS601; Mitosciences, OR, USA), as well as a monoclonal antibody against ANT (MSA02; Mitosciences and rabbit polyclonal antibodies) and mouse 4-HNE (Michael adducts (Calbiochem, San Diego, CA, USA)). Protein specific bands were visualized and quantified with Odyssey Infrared Imager (LI-COR; Wesburg, Leusden, the Netherlands) and expressed as arbitrary units (AU). UCP3 protein band was visualized by chemiluminescence and analyzed by densitometry using Image Master (Pharmacia Biotech, Roosendaal, the Netherlands).

\section{Histological analysis}

The amount of intramyocellular lipids (IMCL) was determined by processing cryosections of tibialis anterior muscle and heart, for Oil Red O staining. Additionally, cell membrane was detected via immunolabeling of the basal membrane marker laminin (Sigma-Aldrich, St. Louis, MO, USA, 1:50 dilution in PBS). IMCL content was expressed per cell surface area [23].

For routine histological analysis, hearts from a subset of mice ( $n=4$ per group) were arrested in diastole, perfusion-fixed with $4 \%$ paraformaldehyde, embedded in paraffin, and sectioned at $5 \mu \mathrm{m}$. Paraffin sections were stained with hematoxylin and eosin (H\&E). Sirius red stainings were performed for the detection of fibrillar collagen.

Changes in apoptosis and cell proliferation in heart (in apex) were determined by analyses of Caspase-3 [24, 25] and Ki-67, respectively. Frozen heart muscle was cryosectioned at $-20^{\circ} \mathrm{C}$ and sections were thaw-mounted on an uncoated glass-slide. Routine immunofluorescence was performed after aceton fixation. Briefly, sections were incubated overnight at $4^{\circ} \mathrm{C}$ with the primary antibodies directed against Cleaved Caspase-3 (Asp175) (Cell Signalling Technology; Bioké, Leiden, the Netherlands) and nuclear Ki-67 (Biocare Medical, Concord, CA, USA), diluted 1:50 and 1:500 respectively in 0.05\% Tween20/TBS (TBST). Thereafter, sections were incubated for $45 \mathrm{~min}$ at room temperature with the appropriate secondary antibody goat anti rabbit AlexFLuor555 (Invitrogen; Groningen, the Netherlands) and $0.5 \mu \mathrm{g} / \mathrm{m} ; 4$ '-6'-diamino-2-phenylindole (DAPI, Invitrogen) for staining of the nuclei. Sections were mounted in Mowiol [15]. All sections were examined using a Nikon E800 fluorescence microscope (Nikon Instruments Europe B.V., Badhoevedorp, the Netherlands) coupled to a Basler A101 C progressive scan color CCD camera. Sections were processed and analyzed using Lucia GF 4.80 software (Nikon, Düsseldorf, Germany). Special care was taken to use the same camera settings (gain and exposure time) while grabbing all images. All images were analyzed for Ki-67 and caspase cleaved 3 positive nuclei. A semi-automatic macro was written that allowed 1) autodetection 
of Oil-red-O, and Ki-67 or cleaved caspase-3 positive nuclei versus 2) all DAPI-stained nuclei.

\section{$\beta-H A D$ and PFK enzyme activity}

Activity of $\beta$-hydroxyacyl-CoA dehydrogenase ( $\beta$-HAD), as a marker for $\beta$-oxidative capacity [26], and phosphofructokinase (PFK) activity, as a marker for glycolysis, were measured in tibialis anterior muscle homogenates. Protein concentration was determined using a Bio-Rad protein assay kit according to manufacturer's instruction. All tissue samples were diluted to $1.5 \mu \mathrm{g} / \mu \mathrm{l}$ in a buffer containing sucrose $(250 \mathrm{mM})$, Tris $(10 \mathrm{mM})$ and EDTA $(2 \mathrm{mM}), \mathrm{pH} 7.4$. The reagent buffer for $\beta-H A D$ activity consisted of tetrapotassiumpyrophosphate $(100 \mathrm{mM}$, $\mathrm{pH} 7.3$ ) and NADH solution (0.002 g in $250 \mu \mathrm{MQ}$ ). Acetoacetyl-CoA (5.0 mg / $2.5 \mathrm{ml}$ distilled water) was used as starting reagent. For PFK analysis, the reagent buffer $(100 \mathrm{ml}, \mathrm{pH} 8.0)$ consisted of $58.5 \mathrm{mM}$ tris-base (Sigma, Zwijndrecht, The Netherlands), $8.9 \mathrm{mM} \mathrm{MgCl}_{2} .6 \mathrm{H}_{2} \mathrm{O}$, $88.8 \mathrm{mM} \mathrm{KCl}, 0.5 \mathrm{mM} \mathrm{KCN}, 3.3 \mathrm{mM}$ ATP, $1.8 \mathrm{mM}$ DTT, $0.37 \mathrm{U} / \mathrm{ml}$ Aldolase (Roche Diagnostics, Mannheim, Germany), $0.51 \mathrm{U} / \mathrm{ml}$ Glycerol-3-phosphate dehydrogenase (Roche Diagnostics), $1.49 \mathrm{U} / \mathrm{ml}$ triose phosphate isomerase (Roche Diagnostics), and $0.29 \mathrm{mM}$ NADH (VWR, Amsterdam, the Netherlands). The reaction was initiated by addition of 5.92 $\mathrm{mM}$ fructose-6-phosphate (Roche Diagnostics) at $37^{\circ} \mathrm{C}$. Measurements were conducted at $340 \mathrm{~nm}, 80$ readings with an interval time of $21 \mathrm{~s}$.

\section{Isolation of skeletal muscle mitochondria}

Isolation of skeletal muscle mitochondria and oxygen consumption measurements were performed as described previously [27, 28]. Briefly, skeletal muscle tissue was trimmed from visible white fat and connective tissue. Proteinase (Subtilisin, $0.7 \mathrm{mg} / \mathrm{g}$ tissue, Sigma-Aldrich) was added and tissue was minced with scissors and homogenized using a mechanical Potter Homogenizer while the tissue was kept $<4^{\circ} \mathrm{C}$. Homogenates were then centrifuged for 10 min at $8500 \mathrm{xg}$ at $4^{\circ} \mathrm{C}$ and supernatant containing floating fat and proteinase was discarded. The remaining pellet was resuspended in isolation medium, homogenized by hand in a Potter Homogenizer and centrifuged at $800 \times \mathrm{g}$ for $10 \mathrm{~min}$ at $4^{\circ} \mathrm{C}$. Subsequently, the resulting supernatant was centrifuged again at $8500 \times \mathrm{g}$ for $10 \mathrm{~min}$ at $4^{\circ} \mathrm{C}$ with the final mitochondrial pellet being gently resuspended by hand homogenization and used for further experiments.

\section{Isolation of cardiac mitochondria}

Cardiac mitochondria were isolated essentially according to the protocol used for skeletal muscle. However, intramyofibrillar (IMF) and subsarcolemmal (SS) mitochondrial fractions were handled separately according to Haemmerle et al. [29]. Freshly isolated cardiac IMF and SS mitochondria $(0.1 \mathrm{mg})$ were used immediately for mitochondrial respirometry. 


\section{Oxygen consumption}

Oxygen consumption rates of freshly isolated mitochondria were measured using a twochamber Oxygraph (Oroboros ${ }^{\circledR}$ Instruments, Innsbruck, Austria). Mitochondria were incubated in a respiration medium containing $100 \mathrm{mM}$ sucrose, $20 \mathrm{mM} \mathrm{K} \mathrm{K}^{+}$TES (pH 7.2), 50 $\mathrm{mM} \mathrm{KCL}, 2 \mathrm{mM} \mathrm{MgCl}_{2}, 1 \mathrm{mM}$ EDTA, $4 \mathrm{mM} \mathrm{KH}_{2} \mathrm{PO}_{4}, 3 \mathrm{mM}$ malate and $0.1 \%$ of fatty acid free BSA. A combination of palmitoyl-L-carnitine $(50 \mu \mathrm{M})$ was used as a fatty acid substrate as well as palmitoyl CoA $(50 \mu \mathrm{M})+$ carnitine $(2 \mu \mathrm{M})$, with the latter substrate being CPT1dependent. Furthermore, Pyruvate $(5 \mathrm{mM})$ was added as a carbohydrate-derived substrate. After addition of the substrates, ADP $(450 \mu \mathrm{M})$ was added to initiate state 3 respiration, followed by oligomycin $(1 \mu \mathrm{g} / \mathrm{ml})$, to block ATP synthesis (state 4 respiration). Maximal oxygen flux (state uncoupled) was obtained by titration of the chemical uncoupler FCCP (carbonyl cyanide p-trifluoromethoxyphenylhydrazone).

\section{Transthoracic echocardiography}

In a subset of WT and UCP3 ${ }^{-/-}$mice ( $\mathrm{n}=4$ per group), cardiac morphology and function were evaluated using high-resolution echocardiography (Vevo 770, VisualSonics, Toronto, Canada) after the 14 days high-fat diet combined with 8 days etomoxir intervention, as described [30]. Left ventricle chamber dimensions were assessed by transthoracic M-Mode in a short-axis view on self-breathing mice under anesthesia ( $2 \%$ isoflurane and $98 \%$ oxygen). Diastolic function was evaluated using pulsed-wave Doppler imaging of the transmitral filling pattern with the early transmitral filling wave (E-wave) followed by the late filling wave due to atrial contraction (A-wave).

\section{Surface Electrocardiogram (ECG)}

A subgroup of mice (WT $n=9, \mathrm{UCP}^{-/} \mathrm{n}=10$ ) was used to measure effects of etomoxir exposure on the electrical activity of the hearts. These mice were also maintained on the high fat diet for 14 days. One hour after i.p. injection of either saline or etomoxir, mice were anesthetized with $1.5 \%$ isoflurane in $95 \% \mathrm{O}_{2}$. A computer based acquisition system (Emka Technologies, Falls Church, VA) was used to record a 6-lead body surface ECG and up to 4 intracardiac electrograms. Fifteen minute recordings were made one hour after injection with (+)-etomoxir sodium salt hydrate $(20 \mathrm{mg} / \mathrm{kg}$ b.w. dissolved in $0.9 \%$ saline $)$. Standard electrophysiological parameters, including the duration of the PR interval, QRS complex, QT interval, and RR interval were measured manually. Corrected QT intervals (QTC) were calculated by the formula QTC $=$ QT $+0.3173(170-R)$ as described [31]. The core temperature of the animal was maintained between $36.5^{\circ} \mathrm{C}$ and $37.5^{\circ} \mathrm{C}$. 


\section{Programmed Electrical Stimulation}

To test for defects in the cardiac conduction system and arrhythmias that are otherwise inapparent. We used programmed electrical stimulation (PES). Mice were anesthetized with $1.5 \%$ isoflurane in $95 \% \mathrm{O}_{2}$, and atrial and ventricular electrograms were recorded using a 1.1F octapolar catheter (EPR-800; Millar Instruments, Houston, TX) inserted via the right jugular vein. Right atrial and ventricular pacing was performed using 2 ms current impulses delivered by an external stimulator (STG-3008, Multi Channel Systems Reutlingen, Germany). Standard electrophysiological protocols were used to determine sinus node recovery time (SNRT), atrioventricular effective refractory period (AVERP), and the ventricular effective refractory period (VERP), which were measured as previously described by $\mathrm{Li}$ and Wehrens [32]. Inducibility of ventricular tachycardia (VT) was determined with single and double extrastimuli and overdrive pacing, each of which were tested twice. Sustained VT was defined as an arrhythmia that self-terminated after 10 or more beats. VT incidence reported reflects sustained VT that occurred following a double extrastimuli protocol in which the S1-S2 interval was progressively reduced by $2 \mathrm{~ms}$ in each pacing train for an interval of $6 \mathrm{~ms}$ greater than to 6 ms less than the VERP. For each value of S2, the S2-S3 interval was progressively reduced in the same manner.

The beta-adrenergic receptor agonist isoproterenol is known to cause cardiac stress and is associated with upregulated UCP3 expression [33]. Therefore, after completion of all protocols, isoproterenol $(0.5 \mathrm{mg} / \mathrm{kg}$, Sigma Aldrich) was administered i.p. to a cohort of mice, and ventricular pacing protocols were repeated to evaluate the importance of UCP3 during cardiac stress along with the inhibitory effect of etomoxir on CPT1. One mouse of each genotype received caffeine $(120 \mathrm{mg} / \mathrm{kg}$, Sigma Aldrich) and epinephrine $(2 \mathrm{mg} / \mathrm{kg}$, Sigma Aldrich) i.p. prior to the repetition of pacing to examine VT inducibility under more stringent conditions.

\section{Statistical analysis}

The data are presented as mean \pm S.E. and statistical analyses were performed using SPSS for Windows 15.0 software (SPSS Inc., Chicago, IL, USA) with statistical significance set at P $<0.05$. An independent student t-test was used to test for differences in UCP3 protein content between saline and etomoxir groups. For all other data genotype, etomoxir intervention, and interaction (genotype*etomoxir) effects were analyzed using a two-way ANOVA (2 $\times 2$ factorial experiment) with univariate analysis of variance. For electrophysiological experiments, continuous variables were expressed as means and evaluated. Categorical data were expressed as percentages and compared with the Fisher's exact test. 


\section{RESULTS}

\section{Lack of UCP3 in relation to skeletal muscle lipotoxicity}

To determine the effect of UCP3 ablation on skm lipotoxicity WT and UCP3 ${ }^{-/-}$mice were fed a high-fat diet for 14 days, and exposed to either saline or etomoxir for 8 days starting at day 6 of the dietary intervention. Body weight $(\sim 27 \mathrm{~g})$ was similar between animal groups, both at the start and at the end of the saline or etomoxir treatment (Table 1 supplemental).

UCP3 protein was not detected in skm of $\mathrm{UCP}^{-/}$mice, regardless of the etomoxirintervention (Figure 1A), confirming their genotype. In WT mice, etomoxir treatment resulted in a 3-fold increase in skm UCP3 protein levels (Figure 1A, $P=0.01$ ). No differences were detected in the protein levels of any of the complexes of the electron transport chain among the groups studied (Table 2 supplemental), implying similar mitochondrial densities in skm of these animals. Also no changes were found in the protein content of the adenine nucleotide transporter (ANT), which has been suggested to be involved in mitochondrial uncoupling (Figure 1B). A trend towards increased ANT levels in WT mice upon etomoxir compared with saline was observed, but this did not reach statistical significance $(P=0.07)$.

Etomoxir inhibits the transport of lipids into mitochondria, and hence the mitochondrial fatty acid oxidation. Increased amounts of intramyocellular lipid (IMCL), e.g. when lipid supply exceeds oxidation, are associated with upregulated UCP3 levels. Therefore, we measured IMCL content. Etomoxir treatment resulted in 4-fold higher lipid accumulation within the TA muscle (Figure $1 \mathrm{C}, \mathrm{P}<0.01$ ), but no effect due to lack of UCP3, or genotype*intervention interaction was found. These data imply that UCP3 does not influence IMCL accumulation under lipid-challenged conditions.

To determine whether UCP3 could lower oxidative stress in skm, we measured 4HNE lipid peroxidation products (Figure 1D). Quantification of lipid peroxidation product levels revealed increased oxidative damage upon etomoxir treatment $(P<0.01)$, but no genotype or genotype*intervention interaction effects were found (Figure 1D), implying that UCP3 does not lower oxidative damage under lipid-challenged conditions.

\section{Skeletal muscle mitochondrial oxidative capacity}

Next, we measured mitochondrial respiration in isolated skm mitochondria. Consistent with an inhibitory effect of etomoxir on CPT1, the etomoxir intervention resulted in a $53 \%$ lower ADP-driven (state 3 ) respiration $(\mathrm{P}<0.01)$ and $57 \%$ lower maximal respiratory capacity (state U) $(P<0.01)$, upon palmitoyl-CoA + carnitine, without a genotype effect (Figure 2A). Interestingly, when using palmitoyl-carnitine as a fatty acid substrate, without requirement of CPT1, etomoxir resulted in a $23 \%$ higher ADP-driven respiration $(P<0.05)$ and a tendency towards higher maximal respiratory capacity $(P=0.06)$, but this effect was not different 


\section{CHAPTER 6}

between genotypes (Figure 2B). With none of the fatty acid substrates, an effect of etomoxir, genotype or genotype*etomoxir interaction on leak (state 40) respiration upon addition of oligomycin (which inhibits ATP synthesis) was detected. ADP-driven respiration in isolated mitochondria, using pyruvate as a glycolytic-derived substrate, was not affected by etomoxir or genotype and no interaction effect was observed (Figure 2C). Also, etomoxir did not alter leak respiration upon pyruvate as a substrate, nor maximal respiratory capacity after addition of the chemical uncoupler FCCP.
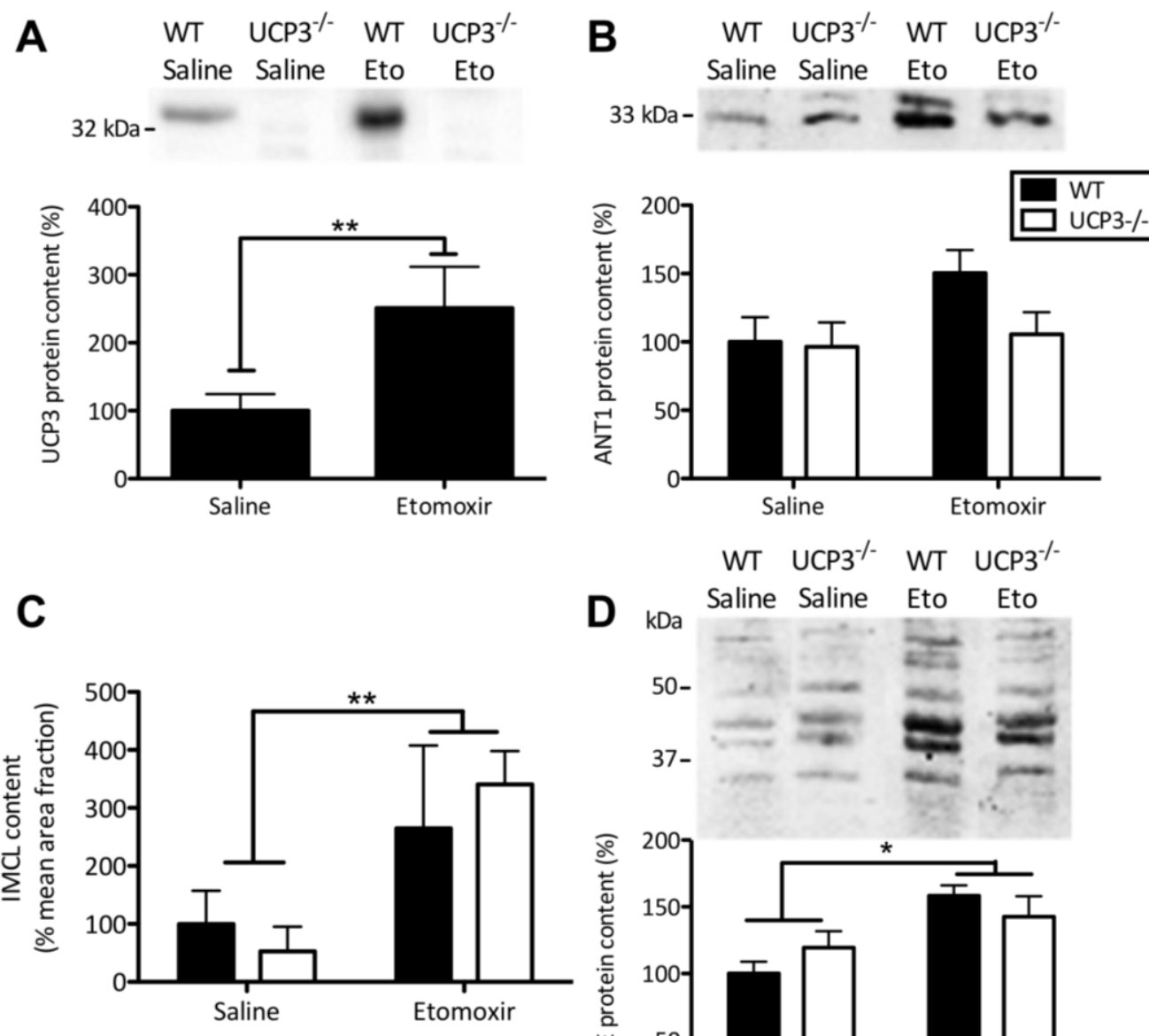

D kDa

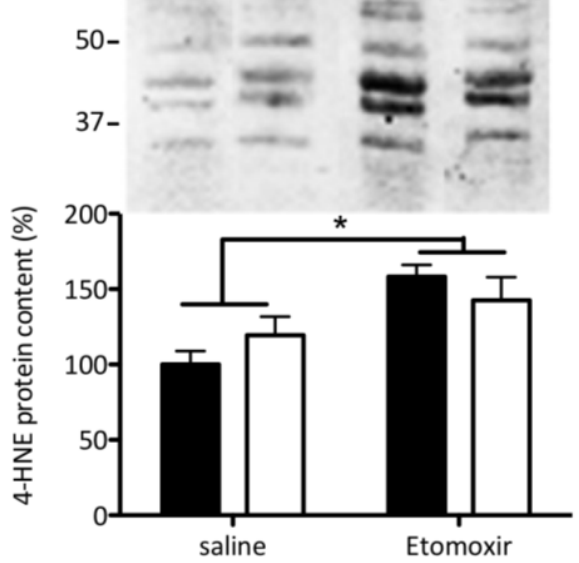

Figure 1. Lack of UCP3 does not lead to skeletal muscle lipotoxicity. Western blots and Oil-red-O stainings were performed in tibialis anterior muscle of WT and UCP3 ${ }^{-/-}$mice. (A) UCP3 protein expression levels in mice. The etomoxir-intervention resulted in an increased UCP3 protein content in WT mice. (B) No effects of etomoxir or lack of UCP3 were found on ANT1 protein levels. (C) In both, WT and UCP3 ${ }^{-/-}$mice, etomoxir significantly elevated intramyocellular lipids (IMCL) content. Values are percentage positive Oil Red $\mathrm{O}$ stained area per mean cell surface area. (D) Elevated levels of 4-HNE protein adducts upon etomoxir-treatment. The mean amounts of protein levels and IMCL content in WT saline-treated mice were set to $100 \%$. ( $n=5-8$ per group) Values are expressed as mean \pm S.E. ${ }^{*} P<0.05,{ }^{* *} P=0.01$ by 2 -way Anova analyses. 

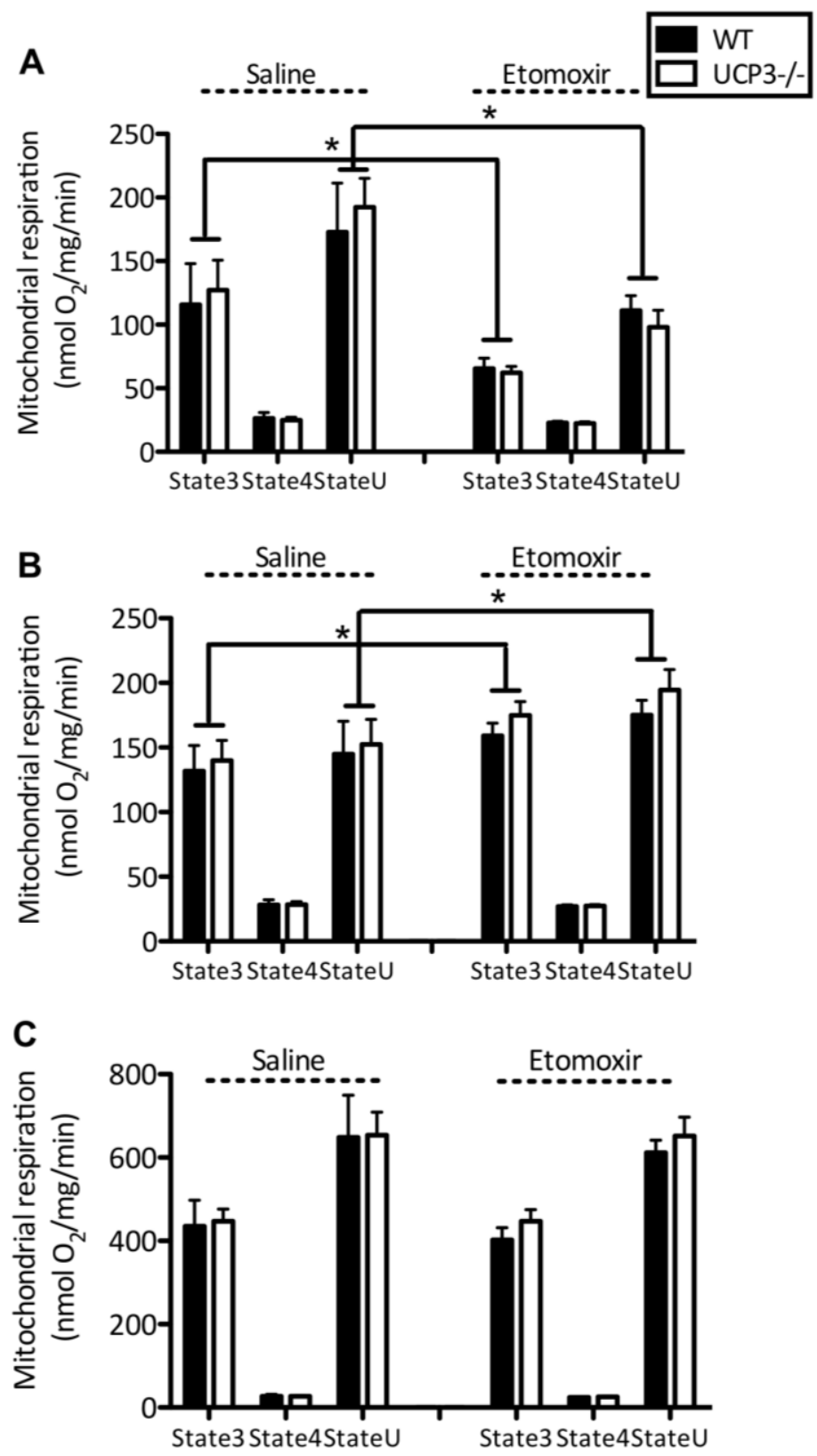

Figure 2. Etomoxir inhibited CPT1dependent mitochondrial respiration upon fatty acid substrates. UCP3 ablation does not affect skeletal muscle mitochondrial oxidative capacity. State 3 , state 4 and state U (maximal respiratory capacity) mitochondrial respiration were monitored in $\mathrm{UCP}^{-/}$and WT mice treated with etomoxir or saline. Palmitoyl CoA + carnitine (A) palmitoyl-carnitine (B) and pyruvate (C) were used as substrates. Mitochondrial protein concentrations were $0.1 \mathrm{mg}$ protein $/ \mathrm{ml}$ for pyruvate and $0.25 \mathrm{mg}$ protein $/ \mathrm{ml}$ for palmitoyl CoA + carnitine and palmitoyl-carnitine. Values are expressed in $\mathrm{nmol} / \mathrm{mg}$ mitochondrial protein/min. $(\mathrm{n}=7-8$ per group) Values are expressed as mean \pm S.E. ${ }^{*} \mathrm{P}<0.05$ by 2 -way Anova analyses.

In line with the respiration data obtained from isolated mitochondria, there were no genotype effects on the maximal activities of $\beta-H A D$ and PFK in skm homogenates, indicating similar skm $\beta$-oxidative and glycolytic capacities in WT and UCP3 ${ }^{-/-}$mice (Figure 1 supplemental).

Taken together, our data do not support a role for UCP3 in the preservation of skm mitochondrial function under lipid-challenged conditions.

\section{Sudden cardiac arrest in $\mathrm{UCP}^{-/-}$mice upon lipid-challenge}

Interestingly, during our initial experiments focused on skm metabolism, we observed that 6 out of $24 \mathrm{UCP}^{-/}$mice died during the first days of etomoxir, an effect that we did not observe in WT mice (Figure 3). Therefore, we expanded the number of animals to further 
investigate underlying mechanisms in the heart. We investigated if intracardiomyocellular lipotoxicity, cell death, alterations in mitochondrial oxidative capacity, left ventricular systolic and diastolic function, or arrhythmias could possibly explain the mortality seen in the UCP ${ }^{-/-}$ mice.

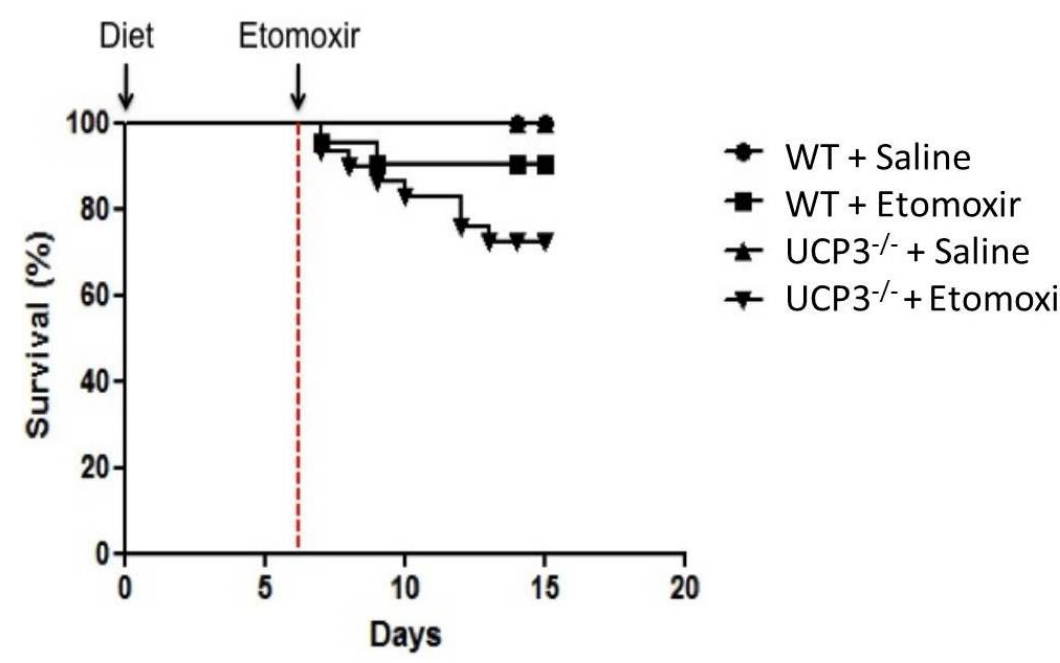

Figure 3. Survival curve. WT and $\mathrm{UCP}^{-/-}$mice were maintained on a high fat diet for a period of 2 weeks. During the last 8 days of the dietary intervention, mice received either saline or etomoxir by daily i.p. injections. All saline-treated animals survived the complete intervention period, whereas 2 out of 19 WT mice and 6 out of $24 \mathrm{UCP}^{-/-}$died upon etomoxir treatment.

\section{Intracardiomyocellular lipotoxicity, apoptosis, fibrosis and proliferation}

Analogous to the increase in skm, etomoxir treatment resulted in a 4-fold increase in UCP3 protein levels in the heart of the WT animals (Figure 4A, $\mathrm{P}<0.05$ ), whereas no UCP3 was detected in $\mathrm{UCP}^{-1-}$ mice. We were able to collect tissue from one of the two WT mice that died upon etomoxir, and interestingly cardiac UCP3 protein content in this etomoxir treated mouse was very low ( 7-fold reduction compared with average cardiac UCP3 protein content in the WT group, data not shown). This might suggest that cardiac UCP3 has beneficial effects on survival rate under lipid-challenged conditions. Therefore, we investigated whether the increased mortality in $\mathrm{UCP}^{-{ }^{-}}$mice upon etomoxir treatment could be due to cardiac lipotoxicity. However, in contrast to skm, inhibition of CPT1 by etomoxir resulted in a 4-fold lowering of lipid content in the heart (Figure $4 \mathrm{~B}, \mathrm{P}<0.01$ ), independent of genotype. No genotype, etomoxir or genotype ${ }^{*}$ etomoxir interaction effects were found for the amount of cardiac HNE lipid peroxidation products (Figure 5E), indicating no difference in oxidative damage between UCP3 ${ }^{-/}$mice compared with their WT littermates. Additionally, we found that etomoxir decreased the percentage of caspase-3 positive nuclei (Figure $4 \mathrm{C}, \mathrm{P}<0.01$ ), suggesting less apoptosis in the hearts of etomoxir-treated animals independent of the genotype. No signs of fibrosis were found as Sirius red staining did not show any abnormalities (data not shown). Ki-67 positive intracardiac cell number was elevated upon etomoxir treatment (Figure 4D, $\mathrm{P}<0.001$ ) indicating increased cell proliferation, but again no genotype or genotype*etomoxir interaction effect was observed. These data indicate that neither cardiac lipid content, apoptosis, fibrosis nor impaired cell proliferation could directly explain the increased mortality in the HF-diet fed $\mathrm{UCP}^{-/}$animals upon etomoxir treatment. 

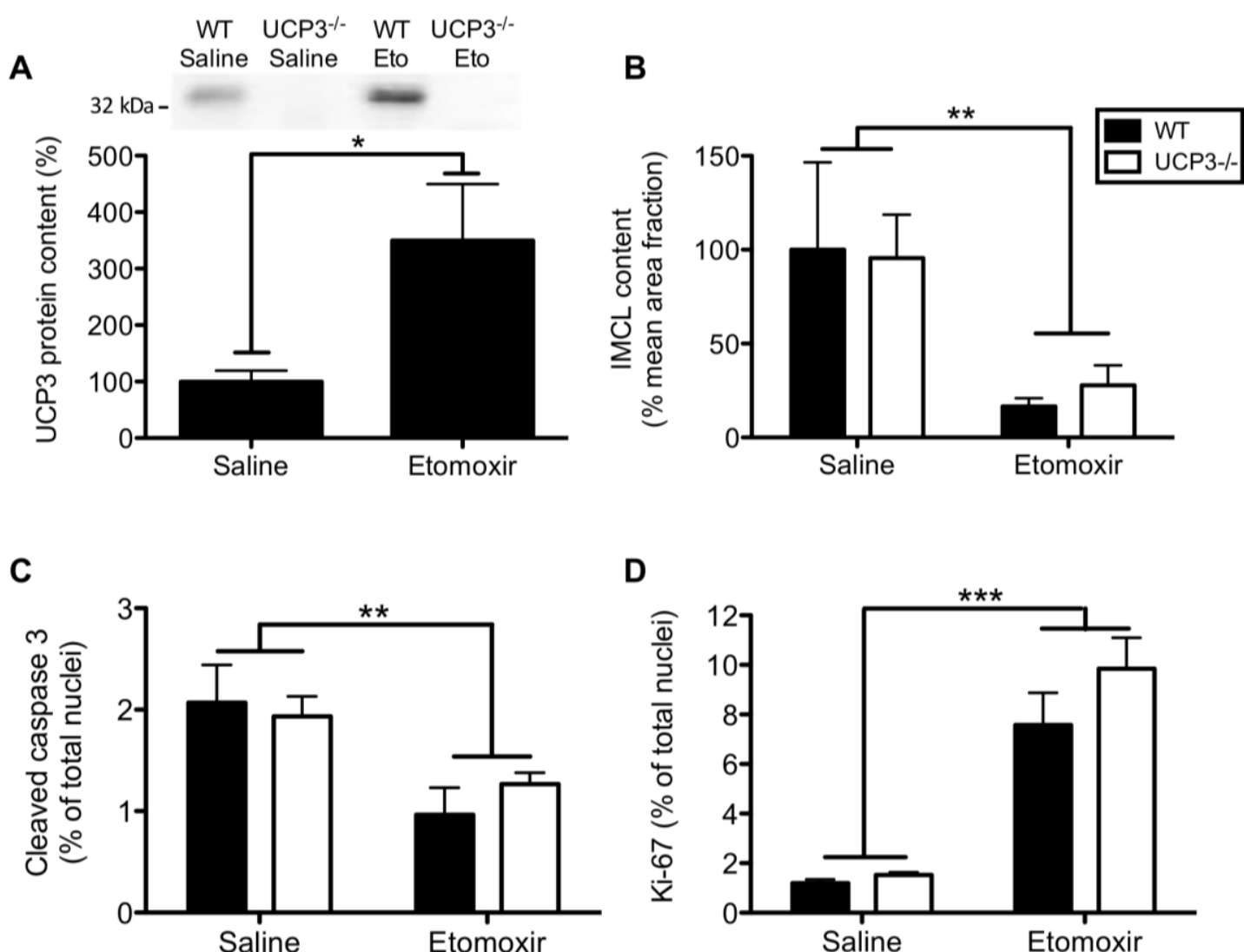

Figure 4. Lack of UCP3 does not lead to cardiac lipotoxicity. (A) Quantification of immunoblot of UCP3 protein in cardiac muscle of mice (WT saline $n=7$, WT etomoxir $n=6$ ). The average amount of UCP3 in saline-treated WT mice was set to $100 \%$. Etomoxir-intervention resulted in an increased UCP3 protein content in WT mice. UCP3 protein was not detected in cardiac tissue of $\mathrm{UCP}^{-/}$mice. Quantification of the histological stained cardiac fractions showed a significant decrease upon etomoxir-treatment in the content of both intramyocellular lipids (IMCL) ( $n=7-8$ per group) (B) as well as the amount of cleaved-caspase 3 positive nuclei ( $n=3-6$ per group) (C). The number of Ki67 positive intracardiac cells increased after etomoxir treatment ( $n=6-8$ per group) (D). IMCL values were analyzed as percentage positive Oil-Red-O stained area per mean cell surface area and are expressed as mean \pm S.E. with WT saline mice set to $100 \%$. The number of nuclei positive for cleaved-caspase-3 was expressed as a percentage of immunopositive nuclei and are expressed as mean \pm S.E. Values are expressed as mean \pm S.E. ${ }^{*} P<0.05$, ${ }^{* *} P<0.01$, and ${ }^{* * *} P<0.001$ by 2 -way Anova analyses.

\section{Cardiac mitochondrial oxidative capacity}

Next we tested if lack of UCP3 affected intrinsic mitochondrial function under lipid-challenged conditions. No significant genotype, etomoxir or genotype*etomoxir interaction effects were detected on the content of any of the complexes of the oxidative phosphorylation system in the heart (Table 3 supplemental), implying similar cardiac mitochondrial densities in all groups. After mitochondrial isolation and separation, the amount of subsarcolemmal (SS) mitochondria was larger in comparison to the amount of intramyofibrillar (IMF) mitochondria $(P<0.001)$, regardless the genotype or intervention (data not shown).

Respirometry measurements in cardiac IMF mitochondria revealed a reduction in ADP-driven (state 3) $(62 \%, \mathrm{P}<0.001)$, leak (state 4o) $(29 \%, \mathrm{P}<0.05)$, and uncoupled (state U) 
(74\%, $\mathrm{P}<0.001)$ mitochondrial respiration upon etomoxir in the presence of palmitoyl-CoA + carnitine (Figure $5 \mathrm{~A}$ ), i.e. requiring CPT1. This reduction in mitochondrial respiration upon etomoxir was irrespective of the genotype, implying that UCP3 does not play a key role in fatty acid oxidation in cardiac mitochondria. Of note, in agreement with previous data [34, 35] we showed that IMF mitochondria exhibit higher respiratory rates compared with SS mitochondria. We could not detect an etomoxir effect in the SS mitochondrial fractions (Figure 5B). In contrast, upon a glycolytic-derived substrate, pyruvate, etomoxir resulted in $58 \%$ higher oxygen consumption in state $3(\mathrm{P}=0.01)$ and $51 \%$ increased maximal respiratory capacity $(P<0.01)$ of SS mitochondria, without any effect in IMF mitochondria (Figure 5C-D). Most important, however, again no genotype or genotype*etomoxir interaction was found.

Overall, our data confirm that etomoxir indeed induces an inhibition of cardiac mitochondrial fat oxidation. However, because the level of inhibition was similar between the mice with and without UCP3, the increased mortality in the UCP3 ${ }^{-/}$mice could not be explained by a lower intrinsic cardiac mitochondrial oxidative capacity.

\section{Cardiac function and remodeling}

We next determined if lack of UCP3 under lipid-challenged conditions affects cardiac function and morphology. To this end, transthoracic echocardiography was performed in a subset of both WT and $\mathrm{UCP}^{-{ }^{-}}$mice. Analysis of echocardiography data (Table 4 supplemental) revealed no significant effect of etomoxir on left ventricle systolic function, assessed by fractional shortening (FS) (Figure 6A) in WT or in $\mathrm{UCP}^{-{ }^{--}}$mice. In line with the data on systolic function, no difference in diastolic function, assessed by E/A ratio (Figure 6B), was found between the groups.

Data on left ventricular mass $(P=0.01$; Figure $6 C)$ as well as $\mathrm{H} \& \mathrm{E}$ staining of cardiac tissue (Figure 6D) showed that the etomoxir-intervention induced hypertrophy in both WT and $\mathrm{UCP}^{-/-}$mice. This result implies that UCP3 does not protect against cardiac hypertrophy under lipid-challenged conditions induced by a HF-diet combined with etomoxir.

\section{UCP3 deficiency predisposes to potentially fatal ventricular arrhythmias}

Since UCP ${ }^{-/}$animals do not develop alterations in cardiac function and/or morphology that could directly explain the increased prevalence of sudden death in $\mathrm{UCP}^{-/-}$mice, we tested if UCP3 deficiency under lipid-challenged conditions results in electrophysiological abnormalities. Therefore, we recorded surface ECG in anesthetized animals one hour after etomoxir injection. We found that $\mathrm{UCP}^{-/}$animals exhibited significantly longer QRS intervals than wild-type mice (Figure 7A-C). Furthermore, QTc intervals were significantly prolonged in $\mathrm{UCP}^{-/-}$mice when compared with WT (Figure 7D). No other electrical parameters recorded were different between the two groups (Table 5 supplemental). These data suggest that 
ablation of UCP3 results in delayed ventricular conduction and prolonged repolarization, both of which could promote arrhythmogenesis and sudden cardiac death.
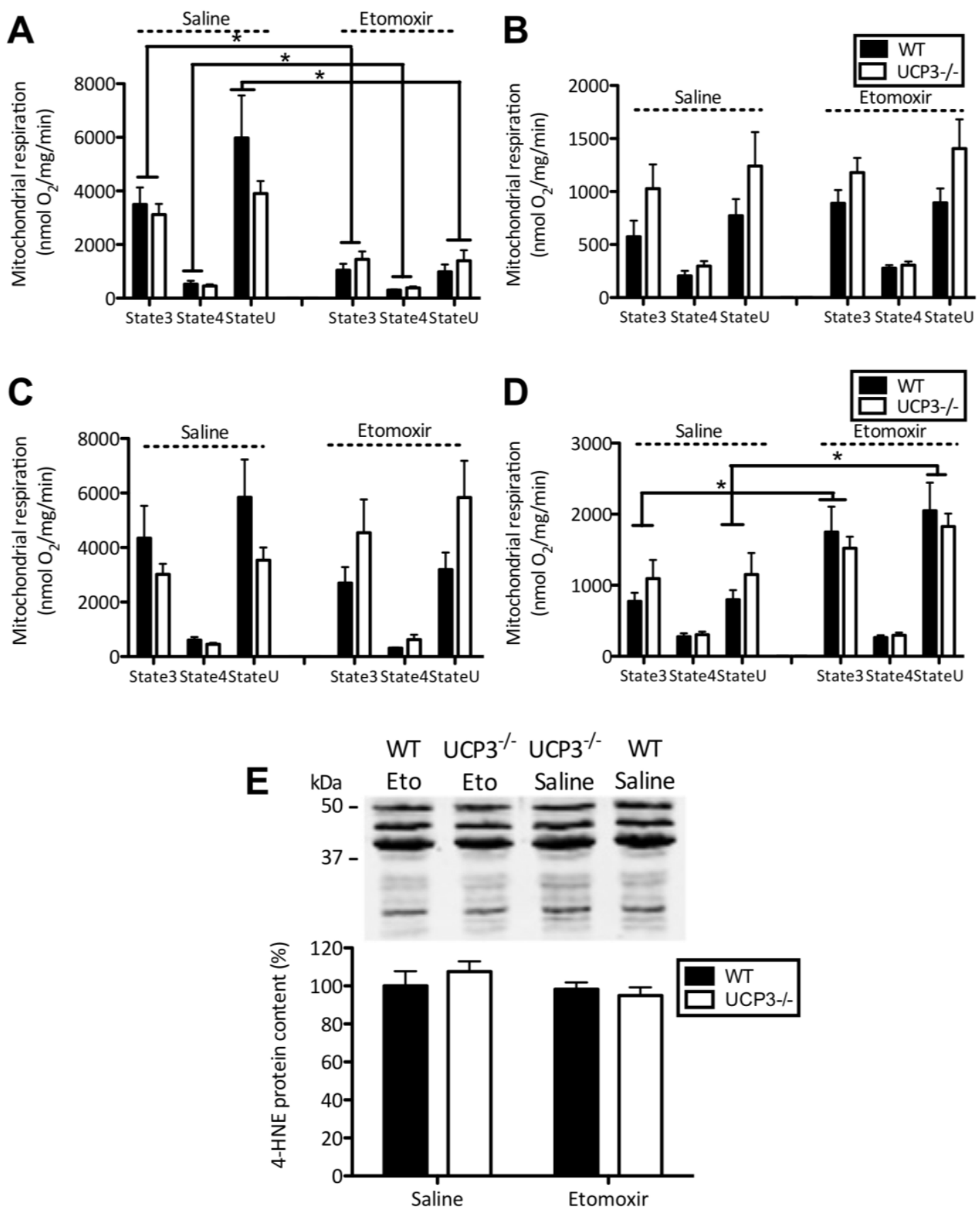

Figure 5. Etomoxir inhibited CPT1-dependent mitochondrial respiration upon fatty acid substrates in IMF fractions. UCP3 ablation does not affect cardiac mitochondrial oxidative capacity. State 3 , state 4 and state $U$ (maximal respiratory capacity) mitochondrial respiration was measured in subsarcolemmal (SS) and intramyofibrillar (IMF) cardiac mitochondria of WT and $\mathrm{UCP}^{-/-}$mice treated with saline or etomoxir. Mitochondrial fractions were separated into IMF (A, C) and SS (B, D) fractions. Palmitoyl CoA + carnitine (A, B) and pyruvate (C, D) were used as substrates. Cardiac mitochondrial protein concentrations were $0.05 \mathrm{mg} / \mathrm{ml}$ for all respiratory measurements. Mitochondrial respiration is expressed as $\mathrm{nmol} / \mathrm{mg}$ mitochondrial protein/min. (E) Representative immunoblot and quantification of 4-HNE protein adducts. Values are expressed as mean \pm S.E. ${ }^{*} P<0.05$, by 2 way ANOVA analyses. ( $n=4-7$ per group). 

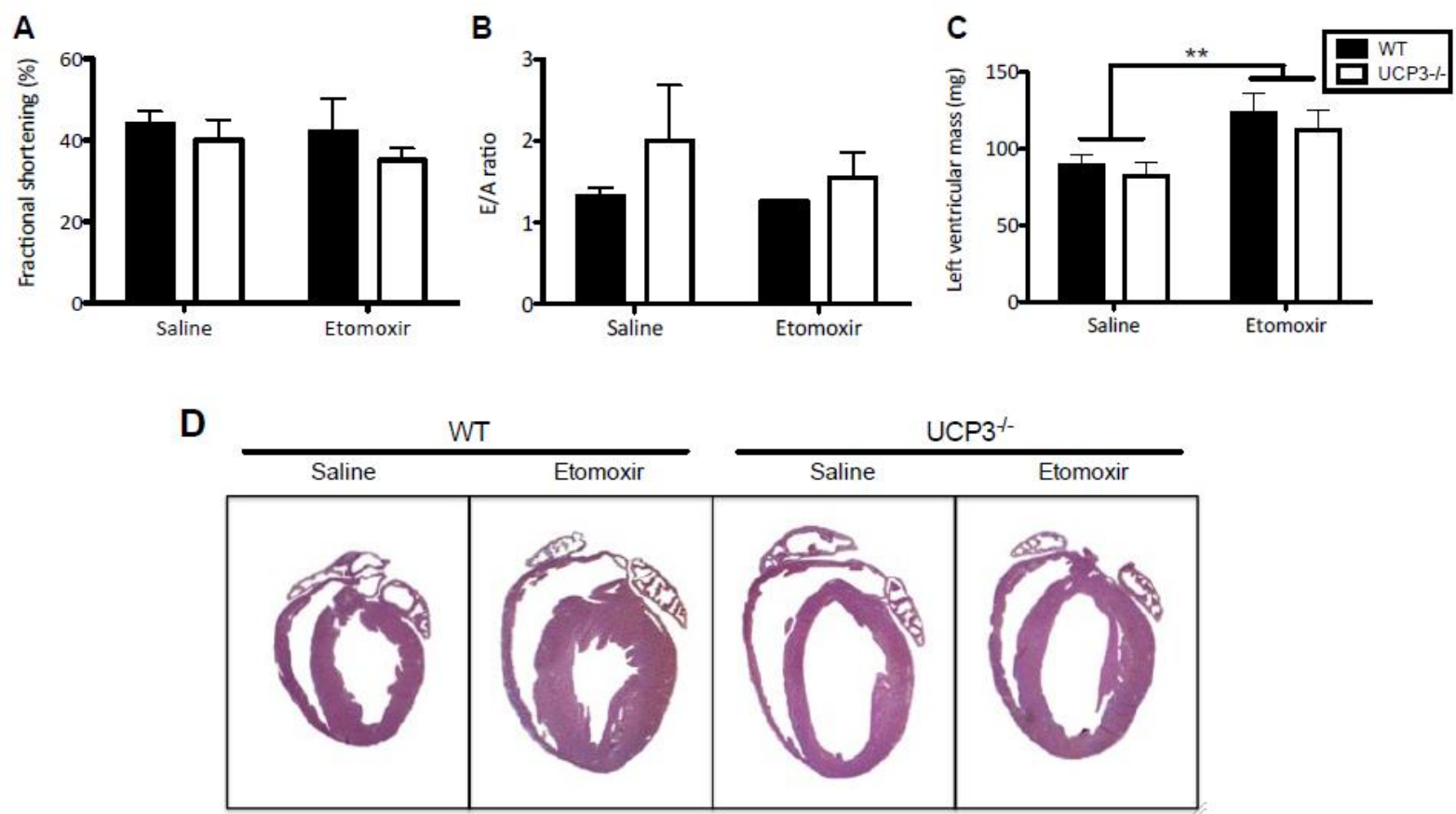

Figure 6. Lack of UCP3 does not affect cardiac function. Transthoracic echocardiography characteristics in WT and $\mathrm{UCP}^{-/}$mice treated with saline or etomoxir of $(\mathbf{A})$ left ventricular fractional shortening (\%), (B) E/A ratio, and (C) left ventricular mass (mg). (D) Representative images of H\&E staining of hearts that were arrested in diastole. Etomoxir treatment for 8 days resulted into cardiac hypertrophy in both, WT and UCP3 ${ }^{-/}$mice. Data are presented as mean \pm S.E. ${ }^{* *} P=0.01$, by 2 -way ANOVA analyses. ( $n=4$ per group).

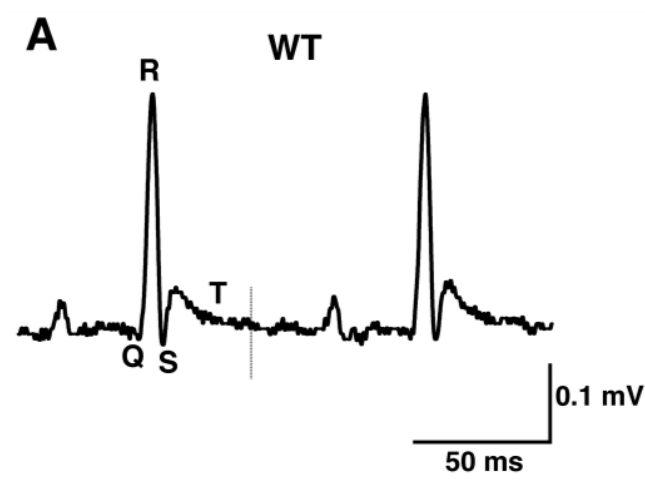

C

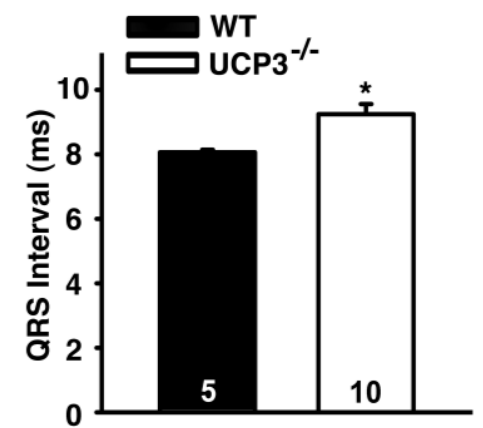

B

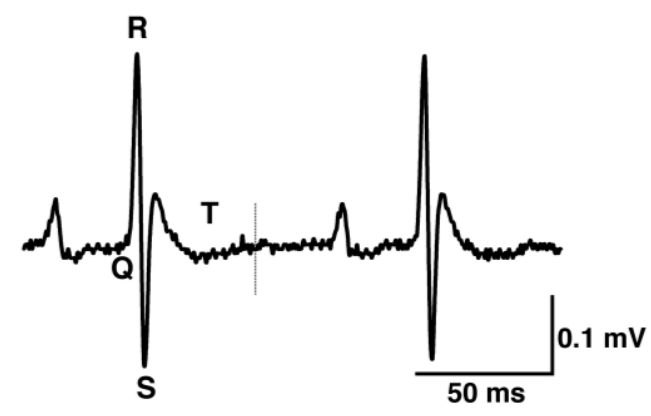

D

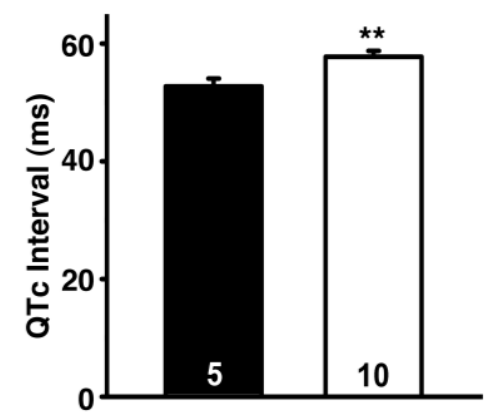

Figure 7. Representative ECG recordings of QRS intervals of WT (A) and UCP3 ${ }^{-1}(\mathbf{B})$ mice. Measurements were made in anesthetized WT and UCP3 ${ }^{-/}$animals at one hour after etomoxir i.p. injection. (C) Quantification of the QRS intervals, and (D) QTc intervals (WT $n=5, \mathrm{UCP}^{-/} \mathrm{n}=10$ ). 
Given that a prolonged QT interval creates a predisposition to arrhythmias, we hypothesized that $\mathrm{UCP}^{-/}$mice are more susceptible to ventricular tachycardia (VT). To investigate this, we performed in vivo intracardiac electrophysiology studies, during which a catheter was inserted into the right ventricle via the right jugular vein. Programmed electrical stimulation (PES) was performed to determine arrhythmia susceptibility in WT and UCP3 ${ }^{-1-}$ mice. Double extrastimuli pacing induced reproducible sustained VT in $60 \%$ of $\mathrm{UCP}^{-/-}$mice compared with $22 \%$ of WT mice (Figure $8 \mathrm{~A}$ ).

PES was repeated after administration of the beta-adrenergic receptor agonist isoproterenol. While $75 \%$ of UCP3 ${ }^{-/}$mice died following pacing (63\% VT/VF; $12 \% \mathrm{AVB}$ ), only $14 \%$ of the WT mice died ( $\mathrm{P}=0.04$; Figure $8 \mathrm{~B}$ ). One $\mathrm{UCP}^{-/-}$mouse experienced spontaneous VT within five minutes of injection that deteriorated into ventricular fibrillation (VF) and lasted for over 1 minute. This arrhythmia episode eventually resulted in asystole (Figure 8C), which suggests that UCP3 deficiency coupled with etomoxir and stress is sufficient to provoke sudden death.

A

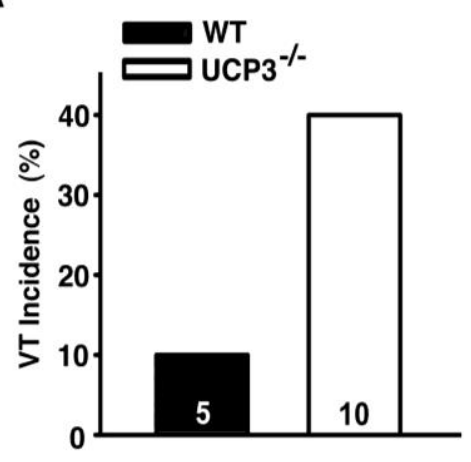

B

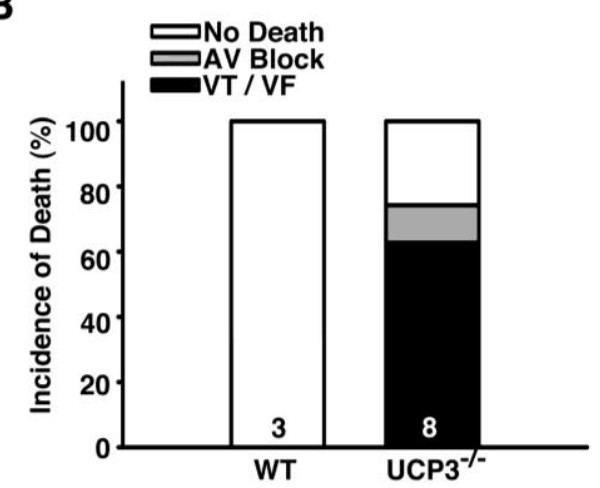

C

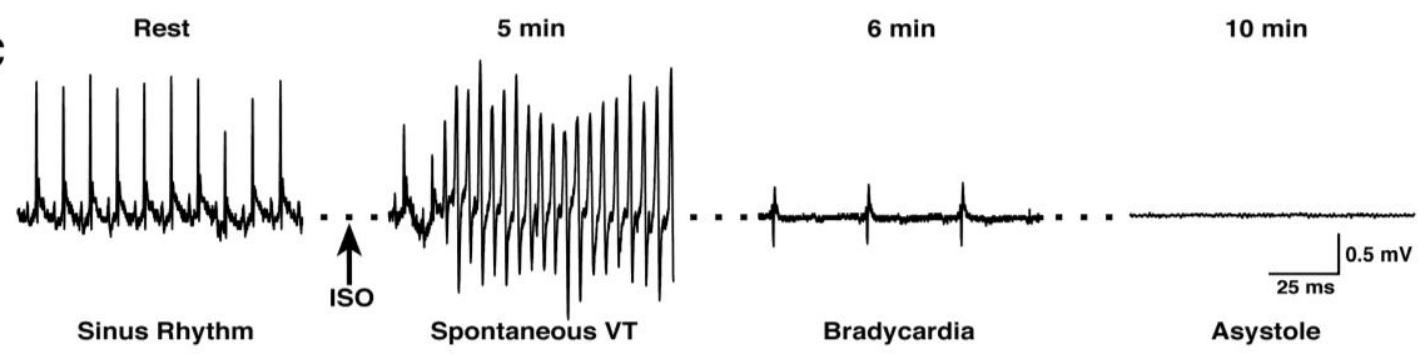

Figure 8. In vivo intracardiac electrophysiology studies in anesthetized WT and UCP3 ${ }^{-/-}$animals at one hour after etomoxir i.p. injection. (A) Double extrastimuli pacing induced reproducible sustained ventricular tachycardia (VT). (B) Incidence of death upon double extrastimuli pacing in the presence of isoproterenol. (C) Recording of arrhythmia that resulted into asystole in $\mathrm{UCP}^{-{ }^{-}}$mouse upon etomoxir. (WT $n=9, \mathrm{UCP} 3^{-/-} n=10$ ).

\section{DISCUSSION}

Mitochondrial uncoupling proteins are involved in the regulation of proton leak over the inner mitochondrial membrane. As such, the best-described uncoupling protein - UCP1 - is able 


\section{CHAPTER 6}

to dissipate energy as heat by increasing proton leak, a mechanism that is crucial for its function in thermoregulation in brown adipose tissue. In 1997, several homologues of UCP1 were identified, of which UCP3 is mainly expressed in skm and heart. So far, the exact physiological function of UCP3 has not yet been revealed, but is suggested to be involved in protection of mitochondrial function, particularly under lipid-challenged conditions [36]. In order to elucidate the role of UCP3, we here examined the effect of UCP3 ablation in a condition in which the dysbalance between the availability of fatty acids and the fatty acid oxidative capacity is maximized. For this, we subjected UCP3 ${ }^{-/}$animals to a HF-diet and combined this with the administration of etomoxir to inhibit fat oxidation. Of note, both etomoxir and HF-diet feeding have been shown to substantially upregulate UCP3 levels [6, 9, $10,22]$. We here report that lack of UCP3 did not identify a significant role for skm UCP3 in protection against lipid-induced mitochondrial dysfunction. However, UCP3 seemed to be crucial for the heart under lipid-challenged conditions as we demonstrated an increased susceptibility to arrhythmias in UCP3 ${ }^{-/}$mice, and an increased risk for sudden cardiac death.

\section{Absence of UCP3 does not affect skeletal muscle lipotoxicity}

To elucidate the role of UCP3 during lipid-challenged conditions, we investigated the effect of etomoxir treatment combined with HF-diet feeding on skm energy metabolism in $\mathrm{UCP}^{-/}$and WT mice. As anticipated, the etomoxir-intervention resulted in increased skm lipid accumulation (4-fold) and a reduction in fat oxidative capacity, without a genotype effect. This data complements a previous study in which we observed that the absence of UCP3 did not alter mitochondrial function upon acute ex vivo exposure of isolated skm mitochondria to palmitate [21]. Based on these previous results, we hypothesized that longer term ablation of UCP3 might reveal its role in the prevention of lipotoxicity. So far many hypotheses about the function of UCP3 suggest mitochondrial protection against lipid-induced stress; e.g. protection against lipotoxicity and oxidative damage via control of reactive oxygen species production [37], or via fatty acid anion transport [38]. However, our results do not support an important beneficial role for skm UCP3 in the protection against skm lipotoxicity. During our experiments on skm metabolism, we did however observe that $25 \%$ of mice lacking UCP3 died shortly after etomoxir administration. This suggests that UCP3 may be more important in the prevention of cardiac lipotoxicity. Indeed, previous studies showed altered cardiac UCP3 expression levels in response to metabolic stress in the heart [39, 40], further supporting our hypothesis. 


\section{Lack of UCP3 does not affect cardiac mitochondrial respiratory capacity}

Preservation of mitochondrial function and structural integrity has been postulated to be essential for cardioprotection [41]. Therefore, to elucidate the role of UCP3 on cardiac metabolism under lipid-challenged conditions, we performed a detailed analysis of cardiac mitochondrial respiratory capacity in $\mathrm{UCP}^{-/-}$and WT mice. As for $\mathrm{skm}$, we confirmed etomoxir administration in mice to be effective as was shown by a $\sim 60 \%$ decline in cardiac mitochondrial respiration upon a separate addition of palmitoyl-CoA and carnitine substrates, in the IMF fraction. Cardiac SS mitochondria showed no etomoxir-induced inhibition of palmitoyl-CoA plus carnitine fuelled mitochondrial respiration. Greater rates of fatty acid oxidation, as well as higher enzymatic activity of CPT1 have previously been shown in rat cardiac IMF mitochondria, compared with SS mitochondria [42] and could explain the observed fractional differences. Etomoxir treatment also led to $\sim 60 \%$ higher respiratory capacity upon pyruvate, but only in SS mitochondria suggesting that respiration in SS mitochondria might be able to compensate for the decreased fatty acid respiratory capacity $[43,44]$. Most important, however, no genotype differences were observed, illustrating that lack of UCP3 does not affect cardiac mitochondrial respiratory capacity, neither on fatty acidnor on glycolytic-derived substrates. Moreover, as we observed higher mortality rates in $\mathrm{UCP}^{-/}$mice, our data imply that cardiac mitochondrial dysfunction is not the main mechanism contributing to sudden cardiac death in these animals.

\section{Lack of UCP3 does not affect cardiac lipotoxicity or cell death}

Interestingly, in contrast to our findings in skm, cardiac lipid content was decreased rather than increased upon etomoxir. Possibly, CPT1b is not the major rate-controlling site in total cardiac long chain fatty acid flux [20]. Again, the etomoxir-effect on cardiac lipid content was independent of the genotype, illustrating that UCP3 does not seem to affect lipid accumulation in the heart. In line with this, lack of UCP3 in cardiac muscle did not affect the accumulation of lipid peroxidation products, a marker of oxidative stress. Also markers for apoptosis, fibrosis or proliferation of connective tissue were not altered in $\mathrm{UCP}^{-/}$mice. Based on these data, we conclude that cardiac lipotoxicity was not the cause for the sudden death in the etomoxir treated UCP $3^{-/-}$mice.

\section{Effect of UCP3 on cardiac functional and electrophysiological characteristics}

Since no increased signs of lipotoxicity or decreased mitochondrial respiratory capacity could be detected in cardiac tissue of $\mathrm{UCP}^{-/}$mice treated with etomoxir, we applied echocardiography in WT and UCP3 ${ }^{--}$mice on a HF-diet with etomoxir. Although these experiments confirmed higher mortality in $\mathrm{UCP}^{-/-}$mice, we could not detect morphological or 
functional differences in systolic or diastolic cardiac function that could explain the sudden cardiac death in these animals.

Cardiac arrhythmias represent a major complication associated with diabetes and they can lead to sudden cardiac death $[45,46]$. Interestingly, it was recently demonstrated that UCP3 expression is required to prevent ischemia reperfusion arrhythmias in addition to preventing contractile dysfunction [36]. This data implies a protective role for UCP3 against cardiac arrhythmias. Although in the present study no systolic or diastolic functional defects were detected, we explored the possibility whether lack of UCP3 could influence the electrophysiological characteristics of the heart after a lipid-challenge. We recorded surface ECGs and found prolonged QTC and QRS intervals in UCP3 ${ }^{-/}$compared with WT mice. QT prolongation has been indicated as an accurate predictor of increased mortality in diabetes patients [47, 48] and is also more frequently reported in obesity [49]. Our data show that upon lipid-challenge, mice lacking UCP3 have an enlarged time interval that is required for completing myocardial depolarization and repolarization. This suggests these mice have an increased susceptibility to arrhythmias, most likely due to (extensive) cardiac remodeling associated with changes in repolarizing currents. Indeed, programmed electrical stimulation experiments supported this theory, as double extrastimuli pacing induced sustained ventricular tachycardia in $60 \%$ of $\mathrm{UCP}^{-/-}$mice compared with $22 \%$ of WT mice.

To our knowledge the present study is the first in vivo study that demonstrates the importance of UCP3 in preventing arrhythmias under lipid-challenged conditions. Diabetes patients are at significantly higher risk of developing cardiomyopathy and heart failure compared with non-diabetics. Although the exact pathogenesis of diabetic cardiomyopathy is still poorly understood, alterations in lipid metabolism have been suggested to be an important mediator [50]. In this context, patients and genetically altered mice with defects in fat metabolism, i.e. infants with very long-chain acyl-CoA dehydrogenase (VLCAD) deficiency [51] and VLCAD null mice [52] have been characterized by prolonged QT intervals, ventricular tachycardia or fatal arrhythmias similar to the etomoxir administered $\mathrm{UCP}^{-/}$mice. In VLCAD null mice abnormal $\mathrm{Ca}^{2+}$ handling is suggested as a possible molecular mechanism of arrhythmias [52]. Given recent hypotheses on a role for UCP3 in cellular $\mathrm{Ca}^{2+}$ handling $[53,54]$, it is tempting to speculate that modulation of repolarization in the $\mathrm{UCP}^{-/-}$mice results from an attempt of cardiomyocytes to maintain $\mathrm{Ca}^{2+}$ homeostasis. Although human data on UCP3 levels in cardiac tissue is limited, UCP3 protein levels are markedly reduced in skm of type 2 diabetes patients [11, 12]. Our present study demonstrates a clear link between disturbed fat metabolism, UCP3 protein levels, and the incidence of cardiomyopathies and sudden death. Future studies should examine if cardiac UCP3 protein levels are low in (diabetes) patients that died from sudden cardiac death. Hence, UCP3 might be a novel therapeutic target for diabetic cardiomyopathy. 
In conclusion, our findings indicate that even under severe in vivo lipid-challenged conditions skm UCP3 does not play a major role in the preservation of skm energy metabolism. Our data in the heart, however, suggest that UCP3 may be crucial in protection against the development of arrhythmias and hence sudden cardiac death. Particularly in light of metabolic diseases like obesity, diabetes and heart failure, it will be interesting to further explore the impact of UCP3 in humans in prevention of arrhythmias under cardiac stress conditions.

\section{Acknowledgements}

We thank Dr. Mary-Ellen Harper, Dr. David Marcinek and Dr. Michael Siegel for kindly providing us with the mice.

\section{Source of funding}

This study was supported by The Netherlands Organization for Health Research \& Development (ZonMw) (Grant No. 9120.6050). M.N. is further supported by the KootstraTalent Fellowship program from the Maastricht University Medical Centre ${ }^{+}$, Maastricht, The Netherlands.

X.H.T.W. is an Established Investigator of the American Heart Association (AHA), and is supported by the Muscular Dystrophy Association, National Institutes of Health (NIH) grants HL089598 and HL091947, and the Fondation Leducq ('Alliance for CaMKII Signaling in Heart').

\section{REFERENCES}

1. Hagen, T., et al., Assessment of uncoupling activity of the human uncoupling protein 3 short form and three mutants of the uncoupling protein gene using a yeast heterologous expression system. FEBS Lett, 1999. 454(3): p. 201-6.

2. Brand, M.D., et al., Oxidative damage and phospholipid fatty acyl composition in skeletal muscle mitochondria from mice underexpressing or overexpressing uncoupling protein 3. Biochem J, 2002. 368(Pt 2): p. 597-603.

3. Russell, A.P., et al., UCP3 protein regulation in human skeletal muscle fibre types I, Ila and IIx is dependent on exercise intensity. J Physiol, 2003. 550(Pt 3): p. 855-61.

4. Schrauwen, P., et al., Skeletal muscle UCP2 and UCP3 expression in trained and untrained male subjects. Int J Obes Relat Metab Disord, 1999. 23(9): p. 966-72.

5. Mingrone, G., et al., Decreased uncoupling protein expression and intramyocytic triglyceride depletion in formerly obese subjects. Obes Res, 2003. 11(5): p. 632-40.

6. Weigle, D.S., et al., Elevated free fatty acids induce uncoupling protein 3 expression in muscle: a potential explanation for the effect of fasting. Diabetes, 1998. 47(2): p. 298-302.

7. Hoeks, J., et al., Peroxisome proliferator-activated receptor-gamma coactivator-1 and insulin resistance: acute effect of fatty acids. Diabetologia, 2006. 49(10): p. 2419-26.

8. Millet, L., et al., Increased uncoupling protein-2 and -3 mRNA expression during fasting in obese and lean humans. J Clin Invest, 1997. 100(11): p. 2665-70. 
9. Schrauwen, P., et al., Etomoxir-induced increase in UCP3 supports a role of uncoupling protein 3 as a mitochondrial fatty acid anion exporter. FASEB J, 2002. 16(12): p. 1688-90.

10. Schrauwen, P., et al., Uncoupling protein 3 as a mitochondrial fatty acid anion exporter. FASEB J, 2003. 17(15): p. 2272-4.

11. Schrauwen, P., et al., Uncoupling protein 3 content is decreased in skeletal muscle of patients with type 2 diabetes. Diabetes, 2001. 50(12): p. 2870-3.

12. Krook, A., et al., Uncoupling protein 3 is reduced in skeletal muscle of NIDDM patients. Diabetes, 1998. 47(9): p. 1528-31.

13. Chow, L., A. From, and E. Seaquist, Skeletal muscle insulin resistance: the interplay of local lipid excess and mitochondrial dysfunction. Metabolism, 2010. 59(1): p. $70-$ 85.

14. Murray, A.J., et al., Uncoupling proteins in human heart. Lancet, 2004. 364(9447): p. 1786-8.

15. Boudina, S., et al., UCP3 regulates cardiac efficiency and mitochondrial coupling in high fat-fed mice but not in leptin-deficient mice. Diabetes, 2012. 61(12): p. 3260-9.

16. Lopaschuk, G.D., G.F. McNeil, and J.J. McVeigh, Glucose oxidation is stimulated in reperfused ischemic hearts with the carnitine palmitoyltransferase 1 inhibitor, Etomoxir. Mol Cell Biochem, 1989. 88(1-2): p. 175-9.

17. Lopaschuk, G.D., et al., Etomoxir, a carnitine palmitoyltransferase I inhibitor, protects hearts from fatty acid-induced ischemic injury independent of changes in long chain acylcarnitine. Circ Res, 1988. 63(6): p. 1036-43.

18. Turcani, M. and H. Rupp, Etomoxir improves left ventricular performance of pressureoverloaded rat heart. Circulation, 1997. 96(10): p. 3681-6.

19. Timmers, S., et al., Augmenting muscle diacylglycerol and triacylglycerol content by blocking fatty acid oxidation does not impede insulin sensitivity. Proc Natl Acad Sci U S A, 2012. 109(29): p. 11711-6.

20. Luiken, J.J., et al., Etomoxir-induced partial carnitine palmitoyltransferase-I (CPT-I) inhibition in vivo does not alter cardiac long-chain fatty acid uptake and oxidation rates. Biochem J, 2009. 419(2): p. 447-55.

21. Nabben, M., et al., Uncoupled respiration, ROS production, acute lipotoxicity and oxidative damage in isolated skeletal muscle mitochondria from UCP3-ablated mice. Biochim Biophys Acta, 2011. 1807(9): p. 1095-105.

22. Hesselink, M.K., et al., Increased uncoupling protein 3 content does not affect mitochondrial function in human skeletal muscle in vivo. J Clin Invest, 2003. 111(4): p. 479-86.

23. Koopman, R., G. Schaart, and M.K. Hesselink, Optimisation of oil red O staining permits combination with immunofluorescence and automated quantification of lipids. Histochem Cell Biol, 2001. 116(1): p. 63-8.

24. Fernandes-Alnemri, T., G. Litwack, and E.S. Alnemri, CPP32, a novel human apoptotic protein with homology to Caenorhabditis elegans cell death protein Ced-3 and mammalian interleukin-1 beta-converting enzyme. J Biol Chem, 1994. 269(49): p. 30761-4.

25. Nicholson, D.W., et al., Identification and inhibition of the ICE/CED-3 protease necessary for mammalian apoptosis. Nature, 1995. 376(6535): p. 37-43.

26. den Hoed, M., et al., Habitual physical activity in daily life correlates positively with markers for mitochondrial capacity. J Appl Physiol, 2008. 105(2): p. 561-8.

27. Nabben, M., et al., The effect of UCP3 overexpression on mitochondrial ROS production in skeletal muscle of young versus aged mice. FEBS Lett, 2008. 582(30): p. 4147-52.

28. Shabalina, I.G., et al., Cold tolerance of UCP1-ablated mice: a skeletal muscle mitochondria switch toward lipid oxidation with marked UCP3 up-regulation not associated with increased basal, fatty acid- or ROS-induced uncoupling or enhanced GDP effects. Biochim Biophys Acta, 2010. 1797(6-7): p. 968-80.

29. Haemmerle, G., et al., ATGL-mediated fat catabolism regulates cardiac mitochondrial function via PPAR-alpha and PGC-1. Nat Med, 2011. 17(9): p. 1076-85. 
30. Dirkx, E., et al., Nfat and miR-25 cooperate to reactivate the transcription factor Hand2 in heart failure. Nat Cell Biol, 2013. 15(11): p. 1282-93.

31. Nuyens, D., et al., Abrupt rate accelerations or premature beats cause life-threatening arrhythmias in mice with long-QT3 syndrome. Nat Med, 2001. 7(9): p. 1021-7.

32. $\mathrm{Li}, \mathrm{N}$. and X.H. Wehrens, Programmed electrical stimulation in mice. J Vis Exp, 2010(39).

33. Joubert, R., et al., Regulation of the expression of the avian uncoupling protein 3 by isoproterenol and fatty acids in chick myoblasts: possible involvement of AMPK and PPARalpha? Am J Physiol Regul Integr Comp Physiol, 2011. 301(1): p. R201-8.

34. Riva, A., et al., Structural differences in two biochemically defined populations of cardiac mitochondria. Am J Physiol Heart Circ Physiol, 2005. 289(2): p. H868-72.

35. Palmer, J.W., B. Tandler, and C.L. Hoppel, Biochemical properties of subsarcolemmal and interfibrillar mitochondria isolated from rat cardiac muscle. J Biol Chem, 1977. 252(23): p. 8731-9.

36. Ozcan, C., et al., Role of uncoupling protein 3 in ischemia-reperfusion injury, arrhythmias, and preconditioning. Am J Physiol Heart Circ Physiol, 2013. 304(9): p. H1192-200.

37. Brand, M.D., et al., Mitochondrial superoxide: production, biological effects, and activation of uncoupling proteins. Free Radic Biol Med, 2004. 37(6): p. 755-67.

38. Schrauwen, P., W.H. Saris, and M.K. Hesselink, An alternative function for human uncoupling protein 3: protection of mitochondria against accumulation of nonesterified fatty acids inside the mitochondrial matrix. FASEB J, 2001. 15(13): p. 2497-502.

39. Murray, A.J., et al., Increased mitochondrial uncoupling proteins, respiratory uncoupling and decreased efficiency in the chronically infarcted rat heart. J Mol Cell Cardiol, 2008. 44(4): p. 694-700.

40. Razeghi, P., et al., Downregulation of metabolic gene expression in failing human heart before and after mechanical unloading. Cardiology, 2002. 97(4): p. 203-9.

41. Rosca, M.G. and C.L. Hoppel, Mitochondria in heart failure. Cardiovasc Res, 2010. 88(1): p. 40-50.

42. Holloway, G.P., et al., In obese Zucker rats, lipids accumulate in the heart despite normal mitochondrial content, morphology and long-chain fatty acid oxidation. J Physiol, 2011. 589(Pt 1): p. 169-80.

43. Ritov, V.B., et al., Deficiency of subsarcolemmal mitochondria in obesity and type 2 diabetes. Diabetes, 2005. 54(1): p. 8-14.

44. Mollica, M.P., et al., Heterogeneous bioenergetic behaviour of subsarcolemmal and intermyofibrillar mitochondria in fed and fasted rats. Cell Mol Life Sci, 2006. 63(3): p. 358-66.

45. Erickson, J.R., et al., Diabetic hyperglycaemia activates CaMKII and arrhythmias by O-linked glycosylation. Nature, 2013. 502(7471): p. 372-6.

46. Bergner, D.W. and J.J. Goldberger, Diabetes mellitus and sudden cardiac death: what are the data? Cardiol J, 2010. 17(2): p. 117-29.

47. Veglio, M., et al., QT interval prolongation and mortality in type 1 diabetic patients: a 5 -year cohort prospective study. Neuropathy Study Group of the Italian Society of the Study of Diabetes, Piemonte Affiliate. Diabetes Care, 2000. 23(9): p. 1381-3.

48. Linnemann, B. and H.U. Janka, Prolonged QTc interval and elevated heart rate identify the type 2 diabetic patient at high risk for cardiovascular death. The Bremen Diabetes Study. Exp Clin Endocrinol Diabetes, 2003. 111(4): p. 215-22.

49. Poirier, P., et al., Obesity and cardiovascular disease: pathophysiology, evaluation, and effect of weight loss: an update of the 1997 American Heart Association Scientific Statement on Obesity and Heart Disease from the Obesity Committee of the Council on Nutrition, Physical Activity, and Metabolism. Circulation, 2006. 113(6): p. 898-918.

50. van de Weijer, T., V.B. Schrauwen-Hinderling, and P. Schrauwen, Lipotoxicity in type 2 diabetic cardiomyopathy. Cardiovasc Res, 2011. 92(1): p. 10-8.

51. Strauss, A.W., et al., Molecular basis of human mitochondrial very-long-chain acylCoA dehydrogenase deficiency causing cardiomyopathy and sudden death in childhood. Proc Natl Acad Sci U S A, 1995. 92(23): p. 10496-500. 
52. Werdich, A.A., et al., Polymorphic ventricular tachycardia and abnormal $\mathrm{Ca} 2+$ handling in very-long-chain acyl-CoA dehydrogenase null mice. Am J Physiol Heart Circ Physiol, 2007. 292(5): p. H2202-11.

53. Graier, W.F., M. Trenker, and R. Malli, Mitochondrial Ca2+, the secret behind the function of uncoupling proteins 2 and 3? Cell Calcium, 2008. 44(1): p. 36-50.

54. De Marchi, U., C. Castelbou, and N. Demaurex, Uncoupling protein 3 (UCP3) modulates the activity of Sarco/endoplasmic reticulum Ca2+-ATPase (SERCA) by decreasing mitochondrial ATP production. J Biol Chem, 2011. 286(37): p. 32533-41. 


\section{SUPPLEMENTAL MATERIAL}

A

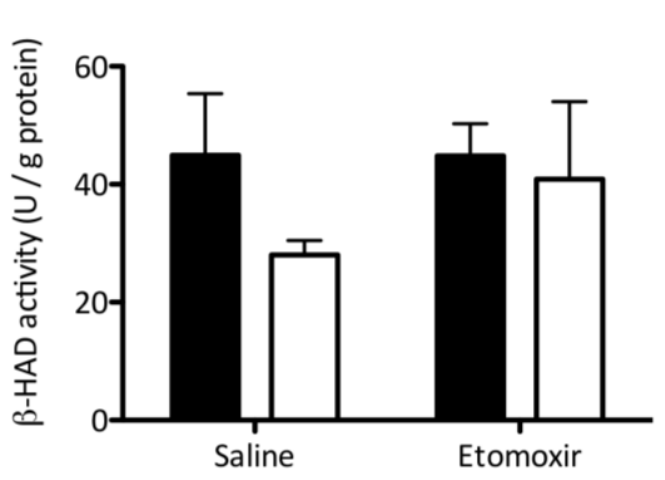

B
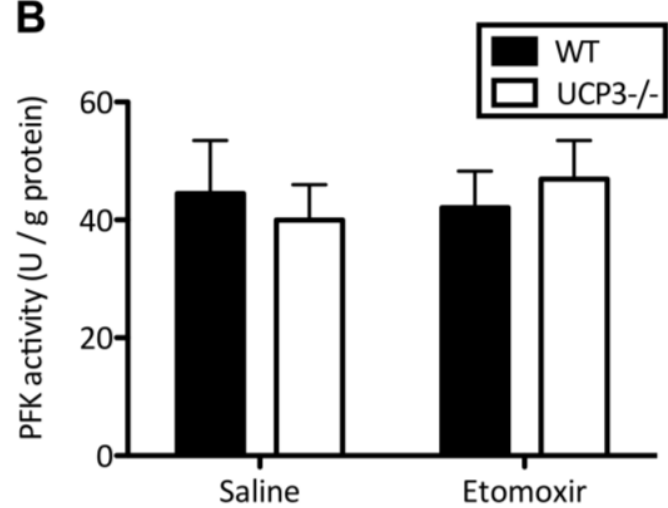

Figure S1. Maximal enzyme activity of $\beta-\operatorname{HAD}(\mathbf{A})$ and PFK (B) in tibialis anterior muscle homogenates. Values are expressed in units per gram protein \pm S.E. ( $\beta$-HAD; WT saline $n=7$, WT etomoxir $n=4$, $\mathrm{UCP}^{-/}$saline $n=5, \mathrm{UCP}^{-/}$etomoxir $\left.n=9\right)\left(P F K ;\right.$ WT saline $n=7$, WT etomoxir $n=7, \mathrm{UCP}^{-/ \text {saline }} n=7$, $\mathrm{UCP}^{-/-}$etomoxir $\left.\mathrm{n}=7\right)$.

Table S1. Body weight of WT and $\mathrm{UCP}^{-/}$mice fed a high fat diet, at day of start (day 6) and end (day 14) of etomoxir /or saline -treatment.

\begin{tabular}{lcccc}
\cline { 2 - 5 } Body weight (g) & \multicolumn{2}{c}{ Saline } & \multicolumn{2}{c}{ Etomoxir } \\
\cline { 2 - 5 } & Wildtype & UCP3 $^{-/-}$ & Wildtype & UCP3 $^{\text {-1- }}$ \\
\hline Day 6 & $26.59 \pm 0.71$ & $26.77 \pm 0.47$ & $27.13 \pm 0.47$ & $27.82 \pm 0.94$ \\
Day 14 (sacrifice) & $26.40 \pm 0.54$ & $26.07 \pm 0.57$ & $26.90 \pm 0.44$ & $27.98 \pm 1.11$
\end{tabular}

Data is expressed as mean \pm S.E. WT saline $n=7$, WT etomoxir $n=7$, UCP3 ${ }^{-1-}$ saline $n=7$, UCP3 ${ }^{-1-}$ etomoxir $n=11$ (body weight at start; at end $n=7$, due to death of 4 mice).

Table S2. Quantification of western blot signal intensity of structural components of the oxidative phosphorylation from skeletal muscle homogenates of WT and UCP3 ${ }^{-/}$mice fed a high fat diet, with or without etomoxir-treatment.

\begin{tabular}{lcccc}
\cline { 2 - 5 } Parameters & \multicolumn{2}{c}{ Saline } & \multicolumn{2}{c}{ Etomoxir } \\
\cline { 2 - 5 } & Wildtype & UCP3 $^{-1-}$ & Wildtype & UCP3 $^{\text {-1- }}$ \\
\hline Complex I & $4.01 \pm 0.57$ & $4.83 \pm 0.52$ & $4.84 \pm 0.68$ & $4.23 \pm 0.16$ \\
Complex II & $5.32 \pm 0.59$ & $6.09 \pm 0.72$ & $5.88 \pm 0.68$ & $5.55 \pm 0.33$ \\
Complex III & $6.40 \pm 0.56$ & $7.28 \pm 0.60$ & $6.65 \pm 0.77$ & $6.32 \pm 0.39$ \\
Complex V & $14.42 \pm 2.80$ & $14.83 \pm 2.49$ & $16.80 \pm 5.31$ & $15.33 \pm 3.81$ \\
\hline Total complexes & $30.15 \pm 3.55$ & $33.03 \pm 3.18$ & $34.17 \pm 6.65$ & $31.43 \pm 4.36$
\end{tabular}

Values are arbitrary units. No significant differences in Oxphos complexes due to lack of UCP3 or etomoxir intervention were established. Data are expressed as mean \pm S.E. ( $n=6-8$ per group). 
Table S3. Quantification of western blot signal intensity of structural components of the oxidative phosphorylation from cardiac muscle homogenates of WT and UCP3 ${ }^{-/-}$mice fed a high fat diet, with or without etomoxir treatment.

\begin{tabular}{|c|c|c|c|c|}
\hline \multirow[b]{2}{*}{ Parameters } & \multicolumn{2}{|c|}{ Saline } & \multicolumn{2}{|c|}{ Etomoxir } \\
\hline & Wildtype & $\mathrm{UCP}^{-1-}$ & Wildtype & $\mathrm{UCP}^{-1-}$ \\
\hline Complex I & $1.56 \pm 0.12$ & $1.66 \pm 0.14$ & $1.59 \pm 0.15$ & $1.85 \pm 0.24$ \\
\hline Complex II & $1.38 \pm 0.08$ & $1.40 \pm 0.13$ & $1.35 \pm 0.11$ & $1.69 \pm 0.35$ \\
\hline Complex III & $1.97 \pm 0.20$ & $1.79 \pm 0.17$ & $1.89 \pm 0.19$ & $2.35 \pm 0.48$ \\
\hline Complex V & $3.01 \pm 0.44$ & $2.89 \pm 0.27$ & $3.36 \pm 0.67$ & $4.22 \pm 1.09$ \\
\hline Total complexes & $7.91 \pm 0.70$ & $7.75 \pm 0.54$ & $8.20 \pm 0.96$ & $10.11 \pm 2.10$ \\
\hline
\end{tabular}

Table S4. Echocardiographic characteristics in WT and UCP3 ${ }^{-/-}$mice after administration of saline or etomoxir.

\begin{tabular}{lcccc}
\cline { 2 - 5 } Parameters & \multicolumn{2}{c}{ Saline } & \multicolumn{2}{c}{ Etomoxir } \\
\cline { 2 - 5 } & Wildtype & UCP3 ${ }^{-/-}$ & Wildtype & UCP3 $^{-/-}$ \\
\hline LVM $(\mathrm{mg})$ & $89 \pm 7$ & $82 \pm 9$ & $123 \pm 13$ & $112 \pm 13$ \\
LVM/BW $(\mathrm{mg} / \mathrm{kg})$ & $3.50 \pm 0.31$ & $3.14 \pm 0.15$ & $4.04 \pm 0.40$ & $3.80 \pm 0.40$ \\
LVM/TL $(\mathrm{mg} / \mathrm{mm})$ & $4.40 \pm 0.74$ & $3.88 \pm 0.10$ & $5.74 \pm 0.56$ & $5.00 \pm 0.65$ \\
Heart rate $($ beats/min) & $513 \pm 12$ & $492 \pm 11$ & $505 \pm 19$ & $487 \pm 33$ \\
LVID, systole $(\mathrm{mm})$ & $1.83 \pm 0.26$ & $2.20 \pm 0.36$ & $2.00 \pm 0.40$ & $2.18 \pm 0.16$ \\
LVID, diastole $(\mathrm{mm})$ & $3.21 \pm 0.31$ & $3.63 \pm 0.27$ & $3.35 \pm 0.26$ & $3.36 \pm 0.12$ \\
IVS, systole $(\mathrm{mm})$ & $1.18 \pm 0.04$ & $1.20 \pm 0.03$ & $1.50 \pm 0.10$ & $1.25 \pm 0.04$ \\
IVS, diastole $(\mathrm{mm})$ & $0.92 \pm 0.07$ & $0.89 \pm 0.02$ & $1.10 \pm 0.10$ & $1.07 \pm 0.06$ \\
LVPW, systole $(\mathrm{mm})$ & $1.68 \pm 0.20$ & $1.26 \pm 0.08$ & $1.70 \pm 0.23$ & $1.57 \pm 0.25$ \\
LVPW, diastole $(\mathrm{mm})$ & $1.08 \pm 0.23$ & $0.75 \pm 0.04$ & $1.25 \pm 0.19$ & $1.12 \pm 0.16$ \\
Ejection fraction $(\%)$ & $82 \pm 3$ & $77 \pm 6$ & $77 \pm 8$ & $73 \pm 3$ \\
Fractional shortening $(\%)$ & $44 \pm 3$ & $40 \pm 5$ & $42 \pm 8$ & $35 \pm 3$ \\
E-wave peak velocity $(\mathrm{m} / \mathrm{s})$ & $811 \pm 13$ & $831 \pm 136$ & $769 \pm 72$ & $540 \pm 90$ \\
A-wave peak velocity $(\mathrm{m} / \mathrm{s})$ & $622 \pm 38$ & $478 \pm 73$ & $610 \pm 48$ & $353 \pm 15$ \\
E/A ratio & $1.32 \pm 0.10$ & $2.00 \pm 0.68$ & $1.26 \pm 0.02$ & $1.55 \pm 0.31$ \\
& & & & \\
\hline
\end{tabular}

$L \overline{V I D}$, left ventricular internal diameter; IVS, interventricular-septum; LVPW, left ventricle posterior wall; LVM/BW, left ventricular mass normalized by body weight; LVM/TL, left ventricular mass normalized by tibia length. Data are expressed as mean \pm S.E. ( $n=3-4$ per group). 
Table S5. Electrophysiological intervals in WT and UCP3 ${ }^{-/-}$mice at 13 weeks of age one hour after administration of etomoxir.

\begin{tabular}{lcc} 
& \multicolumn{2}{c}{ Etomoxir } \\
\cline { 2 - 3 } Parameters & Wildtype & UCP3 $^{-1-}$ \\
\hline PR $(\mathrm{ms})$ & $32.86 \pm 0.88$ & $34.64 \pm 1.27$ \\
QRS $(\mathrm{ms})$ & $8.06 \pm 0.08$ & $9.24 \pm 0.314^{*}$ \\
RR $(\mathrm{ms})$ & $100.39 \pm 2.92$ & $101.75 \pm 2.26$ \\
QTc $(\mathrm{ms})$ & $52.71 \pm 1.34$ & $57.78 \pm 0.98^{* *}$
\end{tabular}

Data are expressed as mean \pm S.E. * $P<0.05,{ }^{* *} P<0.01\left(\right.$ WT $n=5$, UCP3 $\left.{ }^{-/-} \mathrm{n}=10\right)$. 
CHAPTER 7

General Discussion 
Type 2 diabetes mellitus (T2DM) is one of the most common chronic diseases with a rapidly increasing prevalence worldwide and is associated with numerous comorbidities, such as obesity, and heart and vascular diseases, that are all linked with high mortality [1]. One of the recognized characteristics in the early onset of T2DM is insulin resistance. Because skeletal muscle is responsible for the majority of the postprandial insulin-stimulated glucose-uptake, skeletal muscle can be considered the quantitatively most prominent site of insulin resistance. A large diversity of factors has emerged as potential contributors to the progression of skeletal muscle insulin resistance, among them lipid overload, oxidative stress, and mitochondrial (dys)function. Even more, various factors can act in concert to modulate insulin sensitivity, further complicating the identification of the exact underlying cause of T2DM.

\subsection{Skeletal muscle mitochondrial (dys)function and insulin resistance}

In skeletal muscle, mitochondrial oxidative capacity is reported to be reduced by $\sim 40 \%$ in elderly insulin resistant individuals as compared with their healthy matched controls as assessed by ${ }^{13} \mathrm{C} /{ }^{31} \mathrm{P}$ NMR spectroscopy [2]. The importance of mitochondrial dysfunction in the progression of insulin resistance and development of T2DM is further supported by evidence for reduced mitochondrial size and a disturbed oxidative capacity found in skeletal muscle mitochondria of type 2 diabetes patients and their first-degree relatives [3-5]. Mitochondrial oxidative capacity is largely determined by intrinsic mitochondrial function and by mitochondrial content [6-9]. Nowadays, it is still unclear whether mitochondrial dysfunction represents a primary pathological defect or a secondary, physiological adaptation to conditions of insulin resistance. Therefore, the causal link between mitochondrial dysfunction and insulin resistance is currently under debate [10].

Inevitable by-products of mitochondrial oxidative phosphorylation are reactive oxygen species (ROS), which are primarily formed by the direct reduction of oxygen through electrons that escape from the electron transport chain (ETC). Excessive ROS can react with multiple cellular components, such as lipids, proteins, and nucleic acids, which then undergo oxidative modifications, also recognized as oxidative damage. In turn, oxidative damage in skeletal muscle has been suggested to lead to disturbed mitochondrial function, insulin signaling, and eventually progression of insulin resistance [11-14]. This notion is supported by findings in previous studies, which have shown that high-fat diet-induced insulin resistance was linked to skeletal muscle mitochondrial dysfunction as a consequence of hyperglycemia- and hyperlipidemia-induced ROS production [5, 15-17]. The aim of the research presented in this thesis was to investigate the role of mitochondrial ROS production, mitochondrial (dys)function, and muscle oxidative capacity in relation to glucose homeostasis. 


\subsection{Mitochondrial ROS production in relation to insulin sensitivity}

A mechanism to lower ROS-induced oxidative damage, and thereby preserve mitochondrial function, would be by stimulating antioxidant activity. Antioxidant systems can be divided into two classes: 1) enzymatic defenses such as superoxide dismutase (SOD) and catalase; and 2) non-enzymatic defenses such as glutathione (GSH), derivatives of plastoquinone (chapter 2), and nutritional compounds (chapter 4). Genetic rodent models with mitochondrial overexpression of human catalase (MCAT) $[14,18]$ or superoxide dismutase (SOD) [19-21], demonstrated that enhancing enzymatic antioxidant systems could preserve mitochondrial function, lower oxidative stress and improve insulin sensitivity upon a high fat diet [19-21] or during aging [18]. These data support the idea that mitochondrial ROS production is a direct determinant in the progression of insulin resistance. However, non-genetic in vivo studies directly assessing the potential role of oxidative stress in causing muscle mitochondrial dysfunction are still limited. Therefore, we investigated whether targeting mitochondrial ROS production via an orally administered antioxidant could alleviate lipid-induced insulin resistance accompanied by improved mitochondrial function (chapter 2 ). To specifically blunt the mitochondrial production of ROS we used a novel mitochondria-specific antioxidant, the Skulachev ion ( $S k Q$, plastoquinonyl decyltriphenyl-phosphonium). SkQ is a small cationic molecule that specifically targets the IMM [22] where it has been shown to effectively lower oxidative damage $[23,24]$. We first confirmed that oxidative stress was significantly elevated in mice upon a high-fat high-sucrose diet for 16 weeks. As anticipated, this effect was completely blunted by $S k Q$ treatment as was demonstrated by normalized levels of carbonylated proteins, mitochondrial hydrogen peroxide production, and superoxide dismutase activity in skeletal muscle of mice. However, in contrast to our expectations, blunting oxidative stress by specifically targeting mitochondrial ROS production did not beneficially affect muscle mitochondrial function, insulin signaling, or whole-body glucose tolerance. In line with our findings in chapter 2 , a previous study demonstrated that mice fed a high fat diet supplemented with apocynin, an NADPH oxidase (an important source of superoxide in muscle) inhibitor, had blunted ROS production in skeletal muscle, yet no effects on fasted glucose levels or glucose tolerance could be observed [25]. Interestingly, apocynin ameliorated skeletal muscle mitochondrial function in these mice [25], implying that mitochondrial dysfunction does not precede insulin resistance per se in diet-induced diabetic mice [15]. These and our data in chapter 2 are not consistent with previous human and animal studies that suggest that mitochondrial ROS production in skeletal muscle is involved in the pathogenesis of insulin resistance and T2DM. Mechanisms mediating the diverse effects in different studies remain unknown, however, it should be considered that genetic rodent models overexpressing an antioxidative enzyme is a non-physiological approach that 
could result in permanent adaptations in substrate metabolism and ROS production that would otherwise not be observed in vivo. This is exemplified by the finding that glutathione peroxidase 1 (Gpx1) knockout mice were protected from high-fat diet-induced obesity, probably due to suppression of pyruvate dehydrogenase kinase isozyme 4 (PDK4) in muscle, resulting into enhanced whole-body carbohydrate utilization [26]. Thus, genetically altered animals could have an adapted energy metabolism, and moreover, ROS might even enhance insulin sensitivity in these models [26]. Therefore, results obtained from geneticallymodified animal models might be complicated to extrapolate to physiological conditions, and even more, to the human situation.

Next to pharmaceutical antioxidants, also a number of dietary strategies have been examined to reduce oxidative stress by lowering ROS levels [27]. As such, genistein, a nutritional compound mainly present in soybeans, has also been reported to have antioxidant activities [28, 29] (chapter 4). Genistein has been shown to moderate levels of ROS, lipid peroxidation, and to restore enzymatic antioxidant systems in liver and brain tissue of insulin resistant rodents $[28,30]$. The actions of soy isoflavones, such as genistein, appear to depend on the complex interaction of several factors, including its interaction with other protein mixtures present in the diet, and duration and concentration of dosage regimens [31]. Because the food matrix is an important determinant of absorption and bioavailability of nutritional compounds such as genistein [31], dietary components should be taken into account for affecting the bioavailability, and consequently, the effect of genistein. In chapter 4, we attempted to circumvent factors influencing the bioavailability as much as possible by the addition of pure genistein. To evaluate whether genistein could antagonize the progression of the hyperinsulinemic normoglycemic state (pre-diabetes) toward full-blown T2DM by lowering muscle oxidative stress, rats that are prone to develop insulin resistance were supplemented with genistein. Nevertheless, the genistein treatment as reported in chapter 4 did not affect skeletal muscle mitochondrial superoxide anion radical production or levels of oxidative stress, as was reflected by similar levels of superoxide formation and carbonylated proteins respectively in genistein-treated animals compared with animals on a control diet. Analogous to these data on oxidative stress, uncoupling protein 3 (UCP3) protein levels, another postulated protective mechanism against lipotoxicity (chapter 6) suggested to be activated by genistein [32], also remained unaffected upon the genistein intervention. UCP3 is suggested to regulate the proton gradient over the inner mitochondrial membrane, thereby influencing mitochondrial reactive oxygen species production [33, 34], especially under high fat conditions. Interestingly however, in chapter $\mathbf{6}$ we found that lack of UCP3 does not influence oxidative stress in skeletal muscle under lipid challenged conditions. Thus, UCP3 knockout mice $\left(\mathrm{UCP}^{-/}\right.$) were subjected to a high fat diet combined with etomoxir; i.e. an inhibitor of carnitine palmitoyl transferase-1 (CPT-1), thereby inhibiting 
mitochondrial lipid oxidation. As anticipated, the high fat diet and etomoxir-intervention resulted in increased skeletal muscle lipid peroxidation products. However, we observed similar levels of oxidative stress in UCP3 ${ }^{-/}$vs. wildtype (WT) littermates, indicating that UCP3 does not affect oxidative stress levels in skeletal muscle under lipid challenged conditions.

Collectively, the findings in this thesis argue against the hypothesis that diet-induced oxidative stress in skeletal muscle diminishes mitochondrial function, nor inevitably contributes to the pathogenesis of insulin resistance and T2DM.

\subsection{Skeletal muscle oxidative capacity and lipotoxicity}

A sedentary lifestyle along with an increased dietary fat intake results in high amounts of circulating free fatty acids as well as a diminished mitochondrial oxidative capacity, and are hypothesized to play a role in the progression of skeletal muscle insulin resistance [35, 36]. Obese and insulin resistant subjects are characterized by increased ectopic fat accumulation [37]. Excess lipid accumulation in skeletal muscle could result into adverse effects on its function, a phenomenon that is known as lipotoxicity [38]. Although mitochondrial ROS generation does not evidently contribute to muscle insulin resistance (chapters 2 and 4), exercise training and calorie restriction - known to improve muscle oxidative capacity - have been reported to be very effective approaches for the prevention and treatment of insulin resistance and T2DM [39-41]. In general terms, however, obese and type 2 diabetes individuals experience difficulties to adhere to strict life-style interventions such as exercise training or dietary regimes [42]. Therefore, other strategies to stimulate mitochondrial oxidative capacity are warranted.

\subsubsection{Nutritional compounds and mitochondrial oxidative capacity}

Nutritional compounds, such as genistein (chapter 4), could induce beneficial effects on several factors regulating muscle oxidative capacity, including improving mitochondrial metabolism by stimulating mitochondrial biogenesis or by providing substrates for oxidative phosphorylation [43, 44]. Besides regulating oxidative stress levels, genistein has also been postulated to improve mitochondrial oxidative capacity by inducing the expression of genes involved in fatty acid oxidation and mitochondrial biogenesis [45], and stimulating glucose uptake [46]. Also on the whole-body level, beneficial effects on glucose homeostasis have been reported [47-49], implying potential beneficial effects of genistein against the progression of insulin resistance $[50,51]$. In chapter 4 the effect of genistein on muscle oxidative capacity was evaluated in a rat model of progressive insulin resistance and development of T2DM. Skeletal muscle mitochondrial oxidative capacity was established through measuring intrinsic mitochondrial function and density. However, in contrast to the advantageous effects of genistein on muscle metabolism reported in previous studies, we did 
not observe a beneficial role of genistein on skeletal muscle mitochondrial oxidative capacity or on protein levels of structural components of the complexes involved in oxidative phosphorylation. Also intramyocellular lipid content and whole-body glucose homeostasis remained unaffected upon genistein. Therefore, our data demonstrate that genistein is not capable to blunt or alleviate the transition of the hyperinsulinemic normoglycemic state towards advanced insulin resistance by improving skeletal muscle mitochondrial oxidative capacity in rats that are prone to develop T2DM. These findings add to the ongoing controversy regarding the relation between dietary genistein supplementation, muscle oxidative capacity, lipotoxicity, and insulin resistance. In this perspective, age and sex of the individual have been reported to affect the action of genistein [52]; because of the structural similarity between genistein and endogenous estrogens larger effects could be expected in subjects with lowered systemic endogenous estrogens, such as postmenopausal females. In this context, a recent clinical trial demonstrates that one year of treatment with genistein in postmenopausal women, improves several parameters associated with risk for T2DM, such as fasting glucose, insulin, and HOMA-IR [50]. We evaluated the effects of genistein in male rats, therefore, potential effects of genistein in females cannot be excluded based on these experiments.

\subsubsection{Mitochondrial uncoupling and muscle metabolism}

Another mechanism, through which muscle oxidative capacity could be improved, is via mitochondrial uncoupling. It has been shown that resting substrate oxidation in the muscle of endurance-trained individuals was increased without any differences in the basal ATP synthesis rate compared with sedentary subjects [53]. These data imply that increased basal mitochondrial uncoupling could represent an alternative mechanism by which exercise training enhances muscle insulin sensitivity via increased substrate oxidation in the resting state [53]. Hence, mitochondrial uncoupling could be a therapeutic target against (lipidinduced) insulin resistance. One way to induce mitochondrial uncoupling is by chemical compounds such as carbonyl cyanide p-trifluoromethoxyphenylhydrazone (FCCP) and 2,4dinitrophenol (DNP) $[54,55]$. In vivo studies have demonstrated that in nontoxic (low) doses, DNP can prevent oxidative stress [56,57], increase substrate oxidation capacity [57, 58], and elevate expression of genes involved in mitochondrial biogenesis [59, 60]. Hence, DNP administration could be beneficial in the treatment of diabetes by enhancing skeletal muscle substrate metabolism. Therefore, in chapter 3 we evaluated the effect of DNP on glucose tolerance and skeletal muscle metabolism in a genetic mouse model of diabetes. In ex vivo experiments, we confirmed the capacity of DNP to increase mitochondrial respiration that is not coupled to ATP-synthesis (i.e. mitochondrial uncoupling), in a dose-dependent manner. However, oral supplementation of mice with DNP in a maximally tolerated (non-toxic), daily 
dose for 14 days revealed no effects on muscle substrate metabolism, IMCL accumulation or whole-body glucose homeostasis under either healthy or diabetic conditions. In addition, no indications for increased mitochondrial biogenesis were found, in contrast to the postulation that DNP enhances muscle mitochondrial metabolism as an adaptive mechanism for the inefficient ATP production [60,61]. Differences in species, dosage, route and duration of administration, or solvent might contribute to the discrepancies in results between the different studies [57, 62]. The time frame of 2 weeks used in chapter 3 might have been too short to induce detectable metabolic adaptations, which are observed in studies using lifelong exposure to DNP [57]. Additionally, systemic DNP administration has been demonstrated to lead to tissue-selective respiratory effects, probably due to different lipid contents of these tissues [57]. In this context, because of the low lipid content in skeletal muscle of rodents, it could be that orally administered DNP resulted in insufficient accumulation of DNP within the muscle in order to induce effects on metabolism within the experimental settings of chapter 3. Our data argue against a potential beneficial role for DNP in promoting muscle substrate oxidation and insulin sensitivity. However, because we did not demonstrate in vivo uncoupling by a maximum-tolerated dose of DNP, its potential to enhance muscle metabolism by mild mitochondrial uncoupling and antagonize the progression of insulin resistance and T2DM remains to be established. Due to high toxicity of DNP in doses above the therapeutic threshold, DNP is prevented from direct clinical use. Interestingly however, it has been recently shown that treatment with a liver-targeted derivative of DNP (DNPME) reduced hepatic lipid content and improved whole-body insulin sensitivity without DNP-associated toxic effects [63], demonstrating its potential utility as a treatment of T2DM. Therefore, alternatives for or derivatives of DNP to induce mitochondrial uncoupling and/or influence muscle substrate oxidative capacity, intramyocellular lipid accumulation, and insulin sensitivity might be more applicable.

\subsubsection{UCP3 and lipotoxicity in skeletal muscle}

UCP3 is also suggested to play a role in mitochondrial oxidative capacity [34]. Moreover, present data frequently reveal an association between UCP3 levels and the development of T2DM $[64,65]$. In this perspective, we previously showed that UCP3 is downregulated by $50 \%$ in skeletal muscle from subjects with impaired glucose tolerance and in type 2 diabetic patients [66]. Although the exact physiological role of UCP3 has not been established, several studies show a tight regulation of UCP3 expression by free fatty acid levels, supporting a regulating role of UCP3 in fatty acid handling. Decreased UCP3 levels were found under conditions of enhanced fatty acid oxidation as in endurance training [67-69] or weight reduction $[70,71]$ and UCP3 appears to be upregulated when the fatty acid supply exceeds oxidation, eg. upon a high fat diet [72, 73], lipid infusion [74], fasting [75], or 
inhibition of fat oxidation [76-78]. In chapter 6 we therefore examined the role of UCP3 in maintaining mitochondrial function in a state of a maximally disturbed balance between lipid availability and oxidative capacity (as mentioned earlier). Intrinsic mitochondrial function in skeletal muscle under lipid challenged conditions was evaluated by respiratory measurements in skeletal muscle of WT vs. UCP3 ${ }^{-/}$mice. Despite the increased skeletal muscle lipid accumulation and reduced fat oxidative capacity upon the high fat and etomoxirintervention, we observed similar levels of intrinsic mitochondrial function and oxidative stress in WT vs. UCP3 ${ }^{-/}$littermates, indicating that UCP3 does not modulate mitochondrial oxidative capacity or lipotoxicity in skeletal muscle.

\subsubsection{Lipid accumulation and insulin sensitivity}

Although data obtained over the past decade connect diminished mitochondrial oxidative capacity with lipid accumulation in the etiology of obesity, insulin resistance and T2DM [79, 80], mechanisms by which disturbed mitochondrial function stimulate lipid uptake and accumulation within obesity have not been fully established. In this context, the recently identified mitochondrial protein mitoNEET [81] has been suggested to be a powerful regulator of mitochondrial oxidative capacity $[82,83]$. MitoNEET is able to inhibit the electron transport chain, and thereby oxidative capacity, by modulating mitochondrial iron content [84, 85]. Interestingly, it has been reported that despite increased lipid accumulation, adiposespecific mitoNEET overexpression improved insulin sensitivity and whole-body glucose homeostasis [83]. These data imply that MitoNEET might have a beneficial effect on the progression of insulin resistance and type 2 diabetes [86, 87]. Since skeletal muscle also is an important tissue in glucose homeostasis, we evaluated in chapter $\mathbf{5}$ whether mitoNEET in skeletal muscle enhances lipid storage by lowering mitochondrial oxidative capacity through specific overexpression of mitoNEET in tibialis anterior muscle of Wistar rats via in vivo gene electroporation. However, elevated levels of mitoNEET in tibialis anterior did not alter mitochondrial function, substrate oxidative capacity, or IMCL levels. Thus, our data argue against a significant role for mitoNEET in skeletal muscle substrate oxidative capacity or lipid disposition. To investigate whether the mitoNEET protein could be associated with the progression of insulin resistance towards full-blown T2DM, we also measured its expression levels in a rat model of progressive insulin resistance. However, mitoNEET protein content remained unchanged in skeletal muscle during the progression of insulin resistance towards T2DM in these maturating rats. Together, our findings in chapter 5 imply that mitoNEET does not play a relevant role in glucose homeostasis in skeletal muscle. Nevertheless, these findings do not exclude the possibility that skeletal muscle insulin resistance could be linked with improved rather than exacerbated mitochondrial oxidative capacity. Other proteins involved in oxidative pathways or lipid uptake might play a more prominent role in skeletal 
muscle oxidative capacity, lipid accumulation, and glucose homeostasis. As such, mitochondria take up fatty acids from plasma by passive diffusion or active transport via proteins such as carnitine palmitoyl-transferase-1 (CPT1), CD36, and fatty acid binding protein (FABP). A possible underlying mechanism for disturbed entry of fatty acids into the mitochondria could be attributed to defects in fatty acid metabolism at the level of CPT1 [88]. Indeed, we showed in chapter 6 that pharmacological inhibition of CPT1 by etomoxir has been associated with enhanced IMCL accumulation in skeletal muscle. Interestingly, we have shown in a previous study that increased muscle lipid accumulation due to chemically blocking of CPT1 by etomoxir does not hamper insulin signaling [89]. In fact, we and others have demonstrated that glucose oxidation and GLUT4 translocation were increased upon CPT1-inhibition, implying an improved rather than decreased insulin signaling $[89,90]$. These data show that (ectopic) fat accumulation does not necessarily precede the etiology of insulin resistance.

\subsection{Concluding remarks and future perspectives}

Various factors such as impaired mitochondrial function, oxidative stress, and ectopic lipid accumulation could result in metabolic abnormalities, contributing to the progression of insulin resistance and ultimately the development of T2DM. In this thesis we studied the interaction between ROS, mitochondrial oxidative capacity, and glucose homeostasis. Our results indicate that diet-induced enhancement of skeletal muscle oxidative stress neither diminishes mitochondrial function per se, nor evidently contributes to the pathogenesis of insulin resistance and T2DM. However, the question whether mitochondrial dysfunction is either a cause or consequence of insulin resistance is currently still unanswered.

Excessive lipid accumulation in skeletal muscle might be an important factor in the etiology of insulin resistance. Because mitochondria are the primary cellular site for fatty acid oxidation and utilization, strategies to improve mitochondrial oxidative capacity might prevent lipotoxicity and thereby improve insulin sensitivity. It would be of interest to further explore the interaction between mitochondrial oxidative capacity and insulin sensitivity. In that respect, an approach to modulate muscle oxidative capacity and lipid accumulation could be still by mitochondrial uncoupling. Intervention studies using non-toxic alternatives for DNP to induce mitochondrial uncoupling and/or influence muscle substrate oxidative capacity in both, diabetic as well as healthy control groups, might provide more insight in the relation between mitochondrial oxidative capacity and insulin sensitivity. What and in which sequence pathways are affected upon modulated mitochondrial oxidative capacity could be further established by more detailed in vitro studies in muscle tissue, cells, or isolated mitochondria. By obtaining more insight in the role of mitochondrial oxidative capacity in the maintenance 
of insulin sensitivity, more specific therapeutic or even preventive strategies for people who are at risk for T2DM could be developed.

\section{REFERENCES}

1. Roglic, G. and N. Unwin, Mortality attributable to diabetes: estimates for the year 2010. Diabetes Res Clin Pract, 2010. 87(1): p. 15-9.

2. Petersen, K.F., et al., Mitochondrial dysfunction in the elderly: possible role in insulin resistance. Science, 2003. 300(5622): p. 1140-2.

3. Petersen, K.F., et al., Impaired mitochondrial activity in the insulin-resistant offspring of patients with type 2 diabetes. N Engl J Med, 2004. 350(7): p. 664-71.

4. Morino, K., K.F. Petersen, and G.I. Shulman, Molecular mechanisms of insulin resistance in humans and their potential links with mitochondrial dysfunction. Diabetes, 2006. 55 Suppl 2: p. S9-S15.

5. Chow, L., A. From, and E. Seaquist, Skeletal muscle insulin resistance: the interplay of local lipid excess and mitochondrial dysfunction. Metabolism, 2010. 59(1): p. 7085.

6. Sivitz, W.I. and M.A. Yorek, Mitochondrial dysfunction in diabetes: from molecular mechanisms to functional significance and therapeutic opportunities. Antioxid Redox Signal, 2010. 12(4): p. 537-77.

7. Kraegen, E.W., G.J. Cooney, and N. Turner, Muscle insulin resistance: a case of fat overconsumption, not mitochondrial dysfunction. Proc Natl Acad Sci U S A, 2008. 105(22): p. 7627-8.

8. Phielix, E., et al., Lower intrinsic ADP-stimulated mitochondrial respiration underlies in vivo mitochondrial dysfunction in muscle of male type 2 diabetic patients. Diabetes, 2008. 57(11): p. 2943-9.

9. Kelley, D.E., et al., Dysfunction of mitochondria in human skeletal muscle in type 2 diabetes. Diabetes, 2002. 51(10): p. 2944-50.

10. Hoeks, J. and P. Schrauwen, Muscle mitochondria and insulin resistance: a human perspective. Trends Endocrinol Metab, 2012. 23(9): p. 444-50.

11. Rains, J.L. and S.K. Jain, Oxidative stress, insulin signaling, and diabetes. Free Radic Biol Med, 2011. 50(5): p. 567-75.

12. Kumashiro, N., et al., Impact of oxidative stress and peroxisome proliferator-activated receptor gamma coactivator-1alpha in hepatic insulin resistance. Diabetes, 2008. 57(8): p. 2083-91.

13. Bloch-Damti, A. and N. Bashan, Proposed mechanisms for the induction of insulin resistance by oxidative stress. Antioxid Redox Signal, 2005. 7(11-12): p. 1553-67.

14. Anderson, E.J., et al., Mitochondrial H2O2 emission and cellular redox state link excess fat intake to insulin resistance in both rodents and humans. J Clin Invest, 2009. 119(3): p. 573-81.

15. Bonnard, C., et al., Mitochondrial dysfunction results from oxidative stress in the skeletal muscle of diet-induced insulin-resistant mice. J Clin Invest, 2008. 118(2): p. 789-800.

16. Russell, A.P., et al., Lipid peroxidation in skeletal muscle of obese as compared to endurance-trained humans: a case of good vs. bad lipids? FEBS Lett, 2003. 551(1-3): p. 104-6.

17. Houstis, N., E.D. Rosen, and E.S. Lander, Reactive oxygen species have a causal role in multiple forms of insulin resistance. Nature, 2006. 440(7086): p. 944-8.

18. Lee, H.Y., et al., Targeted expression of catalase to mitochondria prevents ageassociated reductions in mitochondrial function and insulin resistance. Cell Metab, 2010. 12(6): p. 668-74.

19. Hoehn, K.L., et al., Insulin resistance is a cellular antioxidant defense mechanism. Proc Natl Acad Sci U S A, 2009. 106(42): p. 17787-92. 
20. Boden, M.J., et al., Overexpression of manganese superoxide dismutase ameliorates high-fat diet-induced insulin resistance in rat skeletal muscle. Am J Physiol Endocrinol Metab, 2012. 303(6): p. E798-805.

21. Liu, Y., et al., Oxidative damage associated with obesity is prevented by overexpression of CuZn- or Mn-superoxide dismutase. Biochem Biophys Res Commun, 2013. 438(1): p. 78-83.

22. Skulachev, V.P., et al., An attempt to prevent senescence: a mitochondrial approach. Biochim Biophys Acta, 2009. 1787(5): p. 437-61.

23. Antonenko, Y.N., et al., Mitochondria-targeted plastoquinone derivatives as tools to interrupt execution of the aging program. 1. Cationic plastoquinone derivatives: synthesis and in vitro studies. Biochemistry (Mosc), 2008. 73(12): p. 1273-87.

24. Bakeeva, L.E., et al., Mitochondria-targeted plastoquinone derivatives as tools to interrupt execution of the aging program. 2. Treatment of some ROS- and age-related diseases (heart arrhythmia, heart infarctions, kidney ischemia, and stroke). Biochemistry (Mosc), 2008. 73(12): p. 1288-99.

25. Yokota, T., et al., Oxidative stress in skeletal muscle impairs mitochondrial respiration and limits exercise capacity in type 2 diabetic mice. Am J Physiol Heart Circ Physiol, 2009. 297(3): p. H1069-77.

26. Loh, K., et al., Reactive oxygen species enhance insulin sensitivity. Cell Metab, 2009. 10(4): p. 260-72.

27. Abete, I., et al., Obesity and metabolic syndrome: potential benefit from specific nutritional components. Nutr Metab Cardiovasc Dis, 2011. 21 Suppl 2: p. B1-15.

28. Mohamed Salih, S., et al., Genistein improves liver function and attenuates nonalcoholic fatty liver disease in a rat model of insulin resistance. J Diabetes, 2009. $1(4)$ : p. 278-87.

29. Vedavanam, K., et al., Antioxidant action and potential antidiabetic properties of an isoflavonoid-containing soyabean phytochemical extract (SPE). Phytother Res, 1999. 13(7): p. 601-8.

30. Valsecchi, A.E., et al., The soy isoflavone genistein reverses oxidative and inflammatory state, neuropathic pain, neurotrophic and vasculature deficits in diabetes mouse model. Eur J Pharmacol, 2011. 650(2-3): p. 694-702.

31. Behloul, N. and G. Wu, Genistein: a promising therapeutic agent for obesity and diabetes treatment. Eur J Pharmacol, 2013. 698(1-3): p. 31-8.

32. Palacios-Gonzalez, B., et al., Genistein stimulates fatty acid oxidation in a leptin receptor-independent manner through the JAK2-mediated phosphorylation and activation of AMPK in skeletal muscle. Biochim Biophys Acta, 2013. 1841(1): p. 132140.

33. Hagen, T., et al., Assessment of uncoupling activity of the human uncoupling protein 3 short form and three mutants of the uncoupling protein gene using a yeast heterologous expression system. FEBS Lett, 1999. 454(3): p. 201-6.

34. Brand, M.D., et al., Oxidative damage and phospholipid fatty acyl composition in skeletal muscle mitochondria from mice underexpressing or overexpressing uncoupling protein 3. Biochem J, 2002. 368(Pt 2): p. 597-603.

35. Roden, M., How free fatty acids inhibit glucose utilization in human skeletal muscle. News Physiol Sci, 2004. 19: p. 92-6.

36. Shulman, G.I., Cellular mechanisms of insulin resistance. J Clin Invest, 2000. 106(2): p. 171-6.

37. Savage, D.B., K.F. Petersen, and G.I. Shulman, Disordered lipid metabolism and the pathogenesis of insulin resistance. Physiol Rev, 2007. 87(2): p. 507-20.

38. Schaffer, J.E., Lipotoxicity: when tissues overeat. Curr Opin Lipidol, 2003. 14(3): p. 281-7.

39. Meex, R.C., et al., Restoration of muscle mitochondrial function and metabolic flexibility in type 2 diabetes by exercise training is paralleled by increased myocellular fat storage and improved insulin sensitivity. Diabetes, 2010. 59(3): p. 572-9. 
40. Heilbronn, L.K., et al., Effect of 6-month calorie restriction on biomarkers of longevity, metabolic adaptation, and oxidative stress in overweight individuals: a randomized controlled trial. JAMA, 2006. 295(13): p. 1539-48.

41. Dube, J.J., et al., Effects of weight loss and exercise on insulin resistance, and intramyocellular triacylglycerol, diacylglycerol and ceramide. Diabetologia, 2011. 54(5): p. 1147-56.

42. Yoon, U., L.L. Kwok, and A. Magkidis, Efficacy of lifestyle interventions in reducing diabetes incidence in patients with impaired glucose tolerance: a systematic review of randomized controlled trials. Metabolism, 2013. 62(2): p. 303-14.

43. Liu, J., et al., Targeting mitochondrial biogenesis for preventing and treating insulin resistance in diabetes and obesity: Hope from natural mitochondrial nutrients. Adv Drug Deliv Rev, 2009. 61(14): p. 1343-52.

44. Hao, J., et al., Mitochondrial dysfunction in the liver of type 2 diabetic Goto-Kakizaki rats: improvement by a combination of nutrients. Br J Nutr, 2011. 106(5): p. 648-55.

45. Palacios-Gonzalez, B., et al., Genistein stimulates fatty acid oxidation in a leptin receptor-independent manner through the JAK2-mediated phosphorylation and activation of AMPK in skeletal muscle. Biochim Biophys Acta, 2013.

46. Ha, B.G., et al., Regulatory mechanism for the stimulatory action of genistein on glucose uptake in vitro and in vivo. J Nutr Biochem, 2012. 23(5): p. 501-9.

47. Ae Park, S., et al., Genistein and daidzein modulate hepatic glucose and lipid regulating enzyme activities in C57BL/KsJ-db/db mice. Life Sci, 2006. 79(12): p. 1207-13.

48. Mezei, O., et al., Soy isoflavones exert antidiabetic and hypolipidemic effects through the PPAR pathways in obese Zucker rats and murine RAW 264.7 cells. J Nutr, 2003. 133(5): p. 1238-43.

49. Lee, J.S., Effects of soy protein and genistein on blood glucose, antioxidant enzyme activities, and lipid profile in streptozotocin-induced diabetic rats. Life Sci, 2006. 79(16): p. 1578-84.

50. Squadrito, F., et al., Genistein in the metabolic syndrome: results of a randomized clinical trial. J Clin Endocrinol Metab, 2013. 98(8): p. 3366-74.

51. Jayagopal, V., et al., Beneficial effects of soy phytoestrogen intake in postmenopausal women with type 2 diabetes. Diabetes Care, 2002. 25(10): p. 170914.

52. Orgaard, A. and L. Jensen, The effects of soy isoflavones on obesity. Exp Biol Med (Maywood), 2008. 233(9): p. 1066-80.

53. Befroy, D.E., et al., Increased substrate oxidation and mitochondrial uncoupling in skeletal muscle of endurance-trained individuals. Proc Natl Acad Sci U S A, 2008. 105(43): p. 16701-6.

54. Parascandola, J., Dinitrophenol and bioenergetics: an historical perspective. Mol Cell Biochem, 1974. 5(1-2): p. 69-77.

55. Harper, J.A., K. Dickinson, and M.D. Brand, Mitochondrial uncoupling as a target for drug development for the treatment of obesity. Obes Rev, 2001. 2(4): p. 255-65.

56. Padalko, V.I., Uncoupler of oxidative phosphorylation prolongs the lifespan of Drosophila. Biochemistry (Mosc), 2005. 70(9): p. 986-9.

57. Caldeira da Silva, C.C., et al., Mild mitochondrial uncoupling in mice affects energy metabolism, redox balance and longevity. Aging Cell, 2008. 7(4): p. 552-60.

58. Hoehn, K.L., et al., Acute or chronic upregulation of mitochondrial fatty acid oxidation has no net effect on whole-body energy expenditure or adiposity. Cell Metab, 2010. 11(1): p. 70-6.

59. Cerqueira, F.M., F.R. Laurindo, and A.J. Kowaltowski, Mild mitochondrial uncoupling and calorie restriction increase fasting eNOS, akt and mitochondrial biogenesis. PLoS One, 2011. 6(3): p. e18433.

60. Rohas, L.M., et al., A fundamental system of cellular energy homeostasis regulated by PGC-1alpha. Proc Natl Acad Sci U S A, 2007. 104(19): p. 7933-8. 
61. Desquiret, V., et al., Dinitrophenol-induced mitochondrial uncoupling in vivo triggers respiratory adaptation in HepG2 cells. Biochim Biophys Acta, 2006. 1757(1): p. 2130.

62. Mhatre, S.S., K.G. Chetty, and D.S. Pradhan, Uncoupling of oxidative phosphorylation in rat liver mitochondria following the administration of dimethyl sulphoxide. Biochem Biophys Res Commun, 1983. 110(1): p. 325-31.

63. Perry, R.J., et al., Reversal of hypertriglyceridemia, fatty liver disease, and insulin resistance by a liver-targeted mitochondrial uncoupler. Cell Metab, 2013. 18(5): p. 740-8.

64. Hagen, T. and A. Vidal-Puig, Mitochondrial uncoupling proteins in human physiology and disease. Minerva Med, 2002. 93(1): p. 41-57.

65. Liu, J., et al., The role of uncoupling proteins in diabetes mellitus. J Diabetes Res, 2013. 2013: p. 585897.

66. Schrauwen, P., et al., Uncoupling protein 3 content is decreased in skeletal muscle of patients with type 2 diabetes. Diabetes, 2001. 50(12): p. 2870-3.

67. Russell, A.P., et al., UCP3 protein regulation in human skeletal muscle fibre types I, Ila and IIx is dependent on exercise intensity. J Physiol, 2003. 550(Pt 3): p. 855-61.

68. Fernstrom, M., M. Tonkonogi, and K. Sahlin, Effects of acute and chronic endurance exercise on mitochondrial uncoupling in human skeletal muscle. J Physiol, 2004. 554(Pt 3): p. 755-63.

69. Schrauwen, P., et al., Skeletal muscle UCP2 and UCP3 expression in trained and untrained male subjects. Int J Obes Relat Metab Disord, 1999. 23(9): p. 966-72.

70. Faraut, B., et al., Capsiate administration results in an uncoupling protein-3 downregulation, an enhanced muscle oxidative capacity and a decreased abdominal fat content in vivo. Int J Obes (Lond), 2009. 33(12): p. 1348-55.

71. Mingrone, G., et al., Decreased uncoupling protein expression and intramyocytic triglyceride depletion in formerly obese subjects. Obes Res, 2003. 11(5): p. 632-40.

72. Hesselink, M.K., et al., Increased uncoupling protein 3 content does not affect mitochondrial function in human skeletal muscle in vivo. J Clin Invest, 2003. 111(4): p. 479-86.

73. Weigle, D.S., et al., Elevated free fatty acids induce uncoupling protein 3 expression in muscle: a potential explanation for the effect of fasting. Diabetes, 1998. 47(2): $p$. 298-302.

74. Hoeks, J., et al., Peroxisome proliferator-activated receptor-gamma coactivator-1 and insulin resistance: acute effect of fatty acids. Diabetologia, 2006. 49(10): p. 2419-26.

75. Millet, L., et al., Increased uncoupling protein-2 and -3 mRNA expression during fasting in obese and lean humans. J Clin Invest, 1997. 100(11): p. 2665-70.

76. Schrauwen, P., et al., Etomoxir-induced increase in UCP3 supports a role of uncoupling protein 3 as a mitochondrial fatty acid anion exporter. FASEB J, 2002. 16(12): p. 1688-90.

77. Schrauwen, P., et al., Uncoupling protein 3 as a mitochondrial fatty acid anion exporter. Faseb J, 2003. 17(15): p. 2272-4.

78. Schrauwen, P. and M.K. Hesselink, The role of uncoupling protein 3 in fatty acid metabolism: protection against lipotoxicity? Proc Nutr Soc, 2004. 63(2): p. 287-92.

79. Lowell, B.B. and G.I. Shulman, Mitochondrial dysfunction and type 2 diabetes. Science, 2005. 307(5708): p. 384-7.

80. Ritov, V.B., et al., Deficiency of electron transport chain in human skeletal muscle mitochondria in type 2 diabetes mellitus and obesity. Am J Physiol Endocrinol Metab, 2010. 298(1): p. E49-58.

81. Colca, J.R., et al., Identification of a novel mitochondrial protein ("mitoNEET") crosslinked specifically by a thiazolidinedione photoprobe. Am J Physiol Endocrinol Metab, 2004. 286(2): p. E252-60.

82. Wiley, S.E., et al., MitoNEET is an iron-containing outer mitochondrial membrane protein that regulates oxidative capacity. Proc Natl Acad Sci U S A, 2007. 104(13): p. 5318-23. 
83. Kusminski, C.M., et al., MitoNEET-driven alterations in adipocyte mitochondrial activity reveal a crucial adaptive process that preserves insulin sensitivity in obesity. Nat Med, 2012. 18(10): p. 1539-49.

84. Lin, J., et al., Crystal structure of human mitoNEET reveals distinct groups of iron sulfur proteins. Proc Natl Acad Sci U S A, 2007. 104(37): p. 14640-5.

85. Wiley, S.E., et al., The outer mitochondrial membrane protein mitoNEET contains a novel redox-active 2Fe-2S cluster. J Biol Chem, 2007. 282(33): p. 23745-9.

86. Zuris, J.A., et al., Facile transfer of [2Fe-2S] clusters from the diabetes drug target mitoNEET to an apo-acceptor protein. Proc Natl Acad Sci U S A, 2011. 108(32): p. 13047-52.

87. Zuris, J.A., et al., NADPH inhibits [2Fe-2S] cluster protein transfer from diabetes drug target MitoNEET to an apo-acceptor protein. J Biol Chem, 2012. 287(15): p. 1164955.

88. Kim, J.Y., et al., Evidence of a malonyl-CoA-insensitive carnitine palmitoyltransferase I activity in red skeletal muscle. Am J Physiol Endocrinol Metab, 2002. 282(5): p. E1014-22.

89. Timmers, S., et al., Augmenting muscle diacylglycerol and triacylglycerol content by blocking fatty acid oxidation does not impede insulin sensitivity. Proc Natl Acad Sci U S A, 2012. 109(29): p. 11711-6.

90. Keung, W., et al., Inhibition of carnitine palmitoyltransferase-1 activity alleviates insulin resistance in diet-induced obese mice. Diabetes, 2013. 62(3): p. 711-20. 


\section{Summary}

\section{Samenvatting}




\section{Summary}

The prevalence of obesity and its associated comorbidities is increasing dramatically worldwide. Obesity is considered a major risk factor for other chronic diseases such as type 2 diabetes mellitus (T2DM). One of the recognized characteristics in the early onset of T2DM is insulin resistance. Although the exact underlying mechanism of insulin resistance is not completely understood, lipid accumulation in skeletal muscle is strongly associated with T2DM. In obesity, caloric intake in excess of total body energy expenditure could result into augmented lipid accumulation, not only in adipose tissues but also in non-adipose tissues such as skeletal muscle. Excess lipid accumulation in skeletal muscle can result in toxic effects on its function and is therefore also referred to as lipotoxicity. Since skeletal muscle is responsible for the majority of the postprandial insulin-stimulated glucose uptake, skeletal muscle can be considered the most important site of insulin resistance. Because mitochondria are the primary cellular site for fatty acid oxidation and utilization, strategies to manipulate aspects of skeletal muscle mitochondrial function could potentially modulate lipid handling and therefore insulin sensitivity. Besides generating ATP, mitochondria inevitably produce reactive oxygen species (ROS). Elevated levels of ROS could result into oxidative damage in skeletal muscle, which in turn has been suggested to lead to disturbed mitochondrial function, insulin signaling, and eventually progression of insulin resistance. Blunting mitochondrial ROS production could be an approach to alleviate insulin resistance by lowering muscle oxidative stress. Thus, modulation of skeletal muscle mitochondrial oxidative capacity and/or oxidative stress could be potential strategies to tackle insulin resistance and T2DM.

In this context, a novel mitochondria-specific antioxidant; the Skulachev ion (SkQ, plastoquinonyl decyltriphenyl-phosphonium), has been reported to effectively reduce oxidative damage. The study in chapter 2 confirmed that $S k Q$ treatment can reduce oxidative stress in skeletal muscle in vitro and under high fat conditions in vivo. However, despite this relief of oxidative stress, SkQ treatment did not ameliorate lipid-induced insulin resistance. These findings illustrate that diet-induced oxidative stress is not necessarily a prerequisite for the development of skeletal muscle insulin resistance.

Another way to lower mitochondrial ROS production is via mitochondrial uncoupling; a process in which the mitochondrial proton gradient is dissociated from ATP production. In addition, mitochondrial uncoupling could enhance metabolic rate and insulin action. However, it is unknown whether mitochondrial uncoupling could be beneficial in the treatment of T2DM. By using the chemical uncoupler 2,4- dinitrophenol (DNP), we investigated in chapter 3 whether chemical induced mitochondrial uncoupling could enhance muscle substrate oxidation and glucose homeostasis under diabetic conditions. Although we 
used the maximal dose of DNP that was tolerated by the animals, no effects on muscle metabolism, lipid accumulation, or glucose tolerance were found. Therefore, based on these data, DNP could not be validated as an appropriate therapy for T2DM.

Various dietary strategies have also been described to beneficially affect insulin sensitivity, by lowering oxidative stress and/or by enhancing muscle metabolism. As such, in type 2 diabetic patients the consumption of soy has been reported to have advantageous effects as it lowers fasting insulin levels. Genistein, the most abundant and active phytoestrogen in soy, has been reported to have antioxidant activities and to improve skeletal muscle oxidative metabolism, thereby stimulating fatty acid handling and glucose uptake. In chapter 4 it was evaluated whether genistein supplementation could antagonize the progression of the hyperinsulinemic normoglycemic state (pre-diabetes) toward full-blown T2DM in Zucker Diabetic Fatty rats. However, feeding genistein to these rats did not lead to improvements in whole-body glucose tolerance, skeletal muscle oxidative stress, or mitochondrial function. These data show that dietary supplementation of pure genistein does not alleviate symptoms associated with T2DM progression in rats.

In adipose tissue and liver, the recently identified mitochondrial protein mitoNEET has been suggested to be a powerful regulator of mitochondrial oxidative capacity, and thereby, of lipid accumulation. However, the function of mitoNEET in skeletal muscle has not been examined before. In chapter 5 we overexpressed mitoNEET specifically in skeletal muscle of rats via in vivo gene electroporation and examined the effects on mitochondrial oxidative capacity and intramyocellular lipid accumulation. However, skeletal muscle specific overexpression of the mitochondrial protein mitoNEET did not result in changes in mitochondrial oxidative capacity and -density, whole-muscle substrate metabolism, or lipid content. In addition, mitoNEET protein expression levels in skeletal muscle were comparable between healthy and diabetic rats. Therefore, no indications were found for a relevant physiological role in skeletal muscle of mitoNEET in the transition from pre-diabetes toward advanced T2DM. These data argue against an important role for mitoNEET in glucose homeostasis in skeletal muscle.

Finally, UCP3 is also suggested to play a role in mitochondrial oxidative capacity and in the regulation of mitochondrial ROS production. Although the exact physiological role of UCP3 has not been established, several studies show a tight regulation of UCP3 expression by free fatty acid levels, supporting a regulating role of UCP3 in fatty acid handling. Chapter 6 describes the role of UCP3 in maintaining mitochondrial function in a state of a maximally disturbed balance between lipid availability and oxidative capacity. For this purpose, wildtype and UCP3-ablated mice were subjected to a high-fat diet combined with a fat oxidation inhibitor (etomoxir). As anticipated, the etomoxir-intervention resulted in increased lipid accumulation in skeletal muscle and a reduction in fat oxidative capacity. However, no 


\section{SUMMARY}

UCP3-related effects on skeletal muscle mitochondrial function or lipotoxicity were established. This reveals that even under severe in vivo lipid-challenged conditions UCP3 does not play a major role in the preservation of skeletal muscle energy metabolism. In the heart, however, it was observed that UCP3 may be crucial in protection against the development of arrhythmias and hence sudden cardiac death. The role of UCP3 in the heart is relatively unexplored and further investigation is required to establish its function in cardiac tissue.

In conclusion, several aspects of mitochondrial function have been addressed in relation to glucose homeostasis. The results of the studies described in this thesis indicate that mitochondrial ROS production does not contribute to the pathogenesis of insulin resistance and T2DM per se. Still, mitochondrial dysfunction may contribute to the development of insulin resistance and T2DM. Additional research is warranted to gain more insight in the role of mitochondrial oxidative capacity in the maintenance of insulin sensitivity. 


\section{Samenvatting}

Wereldwijd neemt het aantal mensen met obesitas nog steeds toe. Obesitas is een belangrijke risicofactor voor de ontwikkeling van andere chronische ziekten zoals type 2 diabetes mellitus (T2DM). Insuline resistentie wordt algemeen erkend als een belangrijk voorstadium van T2DM. De onderliggende mechanismen van insuline resistentie zijn nog niet bekend, maar opstapeling van vetten in de spier wordt vaak in verband gebracht met het ontstaan van insuline resitentie. Bij obese, inactieve mensen kan een overmatige inname van vet en energie resulteren in een verhoogde vetstapeling. Uit onderzoek blijkt dat dit overmatige vet niet alleen in het vetweefsel zal worden opgeslagen, maar ook in andere organen zoals de spieren. Een verhoging van vetstapeling in de spier kan vervolgens interfereren met allerlei processen binnen in de spier, waaronder de insulinesignalering. Hierdoor worden de spieren minder gevoelig voor insuline en neemt de capaciteit van spieren om glucose uit het bloed op te nemen af. Op de lange termijn kan dit er toe leiden dat de bloed glucose waarden worden verhoogd en er dus diabetes ontstaat. Deze schadelijke effecten van vetten in de spier wordt ook wel lipotoxiciteit genoemd. Mitochondriën zijn de belangrijkste organellen in de cel waar vetverbranding plaats vind. Door de vetverbranding in de mitochondriën te stimuleren, zou de vetstapeling in de spieren kunnen dalen, en daarmee ook de insuline gevoeligheid en capaciteit om glucose uit bloed op te nemen kunnen verhogen. Mitochondriën functioneren als de energiecentrales van de cel. Mitochondriën verbranden de vetten en koolhydraten uit de voeding en leggen de vrijgekomen energie vast in ATP. Naast ATP worden echter ook reactieve zuurstofdeeltjes (zuurstofradicalen, ROS) gevormd. Overmatige hoeveelheden ROS kunnen oxidatieve schade aanbrengen aan verschillende componenten in de cel, zoals aan DNA en eiwitten. Deze schade kan leiden tot een verstoorde mitochondriële functie, wat vervolgens weer vetstapeling in de spier zou kunnen bevorderen, waardoor de insuline gevoeligheid afneemt. ROS zouden dus een belangrijke rol kunnen spelen in het ontstaan van T2DM. Door de ROS die door mitochondriën worden geproduceerd weg te vangen met behulp van bijvoorbeeld een antioxidant, zou de oxidatieve schade in de cel verlicht kunnen worden en daarmee ook de insuline gevoeligheid van de spier. Kortom, het stimuleren van de mitochondriële vetverbranding en/of verlaging van ROS in de spier kunnen potentiële strategiën zijn om insuline resistentie en T2DM te bestrijden.

In hoofstuk 2 is er gebruik gemaakt van een antioxidant, het Skulachev ion (SkQ), waarvan bekend is dat het specifiek de mitochondriële ROS weg vangt en hiermee de oxidatieve schade beperkt. SkQ bleek inderdaad in staat de oxidatieve schade in de spier te verlagen, zowel in gekweekte spiercellen als in muizen. Desondanks resulteerde deze verlaging van oxidatieve schade niet in een verbeterde insuline gevoeligheid. Deze data 


\section{SUMMARY}

tonen aan dat oxidatieve schade veroorzaakt door mitochondriële ROS niet per se een vereiste is voor de ontwikkeling van insuline resistentie in de skeletspier.

Een andere manier om de ROS productie in mitochondriën te verlagen is door stimulatie van zogenaamde 'mitochondriële ontkoppeling'. Mitochondriële ontkoppeling is een proces waarbij de verbranding van vetten en koolhydraten uit voeding niet meer volledig efficiënt gekoppeld is aan ATP productie, met als gevolg dat deze energie als warmte verloren gaat. Of mitochondriële ontkoppeling ook gunstige effecten heeft op insuline gevoeligheid en het vermogen van de spier om glucose uit het bloed op te nemen, is nog onbekend. Een manier om mitochondriële ontkoppeling te induceren, is via de chemische stof 2,4-dinitrophenol (DNP). In hoofdstuk 3 werd onderzocht of DNP effecten heeft op de verbranding van vetten en koolhdraten in de spier, vetstapeling, en op glucose tolerantie in zowel gezonde als in diabete muizen. Echter, de toediening van DNP had geen effect op de hiervoor genoemde parameters. Hieruit kan worden geconcludeerd dat DNP geen geschikt middel is om het vermogen van de spier om glucose op te nemen te verbeteren.

Voeding kan ook een belangrijke rol spelen in het handhaven van gezonde glucose bloedwaarden. Van verschillende voedingscomponenten is bekend dat deze een positief effect kunnen hebben op de ROS productie of de vetverbranding. Een van die compontenten is genistein, een belangrijk bestanddeel van soja producten. In hoofdstuk 4 werd bestudeerd of supplementatie van genistein de ontwikkeling van T2DM kan tegengaan. Om dit te onderzoeken hebben ratten die een genetische aanleg hebben om T2DM te ontwikkelen, genistein via hun voeding toegediend gekregen. De gebruikte dosering genistein leidde in deze ratten echter niet tot veranderingen in glucose tolerantie, oxidatieve schade, of mitochondriële functie.

Recent is er een nieuw mitochondrieel eiwit ontdekt; mitoNEET, waarvan met name in vetweefsel en lever is aangetoond dat het een belangrijke rol kan spelen in de regulatie van de vetverbranding en daardoor ook invloed kan hebben op de vetstapeling. Om de rol van mitoNEET in de spier te kunnen bestuderen, is in hoofdstuk $\mathbf{5}$ mitoNEET tot overexpressie gebracht in de skeletspieren van ratten door middel van een in vivo gen elektroporatie procedure. In dit hoofdstuk is het effect van een verhoogde hoeveelheid mitoNEET eiwit op de vet- en glucose verbranding in mitochondriën (oxidatieve capaciteit) en op de vetstapeling in de spier bestudeerd. Op basis van de resultaten kan geconcludeerd worden dat spier-specifieke overexpressie van mitoNEET geen veranderingen teweeg brengt in mitochondriële oxidatieve capaciteit, verbranding van vetten en glucose, of in vetstapeling in de spier. Daarnaast vonden wij geen verschillen in mitoNEET eiwit levels in de skeletspieren van gezonde ratten in vergelijking tot diabete ratten. Daarmee zijn er geen aanwijzingen gevonden voor een relevante rol van mitoNEET in de skeletspier tijdens het ontstaan van T2DM. 
Een ander eiwit dat mogelijk een rol speelt in de regulatie van de mitochondriële oxidatieve capaciteit is ontkoppelingseiwit-3 (UCP3). Daarnaast wordt van UCP3 gesuggereerd dat het ook de mitochondriële ROS roductie kan beïnvloeden. Hoewel de exacte fysiologische rol van UCP3 nog onbekend is, is al wel aangetoond dat de expressie van UCP3 nauw samenhangt met de vraag naar - en het aanbod van - vrije vetzuren. Bij grote hoeveelheden vet in de spier, is er ook meer UCP3 aanwezig. Deze data duiden op een mogelijke rol voor UCP3 in de vetstofwisseling. In hoofdstuk 6 is de rol van UCP3 onderzocht in de handhaving van mitochondriële functie tijdens vetstapeling. De balans tussen vet aanvoer en -verbranding werd maximaal verstoord door muizen op een hoog vet voeding te zetten, en de vetverbranding te remmen door etomoxir. Om de rol van UCP3 te onderzoeken, hebben we het effect van de interventie onderzocht in normale muizen, en dit vergeleken met UCP3 ${ }^{--}$muizen, waarin het eiwit UCP3 ontbreekt. Omdat het effect van de hoog vet voeding in combinatie met etomoxir behandeling vergelijkbaar was in muizen met en zonder UCP3, duiden onze resultaten er op dat UCP3 geen voorname rol speelt in handhaving van mitochondriële functie in skeletspier onder hoog vet condities. Min of meer bij toeval ontdekten we echter dat UCP3 in het hart betrokken lijkt te zijn bij het ontstaan van hartritmestoornissen.

Samengevat blijkt uit de resultaten van dit proefschrift dat mitochondriële ROS productie niet per se bijdraagt aan de ontwikkeling van insuline resistentie en T2DM. De capaciteit van mitochondriën om vet te verbranden lijkt wel belangrijk te zijn in het ontstaan van T2DM, maar het onderliggende mechanisme is nog steeds niet bekend. Om meer inzicht te krijgen in het verband tussen mitochondriële functie en insuline gevoeligheid, is meer onderzoek nodig. 


\section{Valorization}




\section{Valorization}

\section{Social relevance}

Diabetes is a chronic disease that occurs either when the pancreas does not produce enough insulin (type 1 diabetes) or when the body does not respond to the hormone insulin properly (type 2 diabetes). Insulin regulates the uptake of glucose from the plasma into skeletal muscle. Type 2 diabetes (T2DM) comprises $90 \%$ of people with diabetes around the world. The prevalence of T2DM is increasing dramatically and reaches epidemic proportions worldwide. The World Health Organization (WHO) predicts that 350-400 million people will suffer from T2DM in the year 2030. Left untreated, diabetes can cause damage to organs and tissues such as the heart, blood vessels, eyes, kidneys, and nerves. T2DM is associated with numerous comorbidities, such as cancer, and heart and vascular diseases, that are all linked with high mortality. These health issues can dramatically impair quality of life, and have an enormous impact on health care costs.

Equally alarming as the increasing prevalence of diabetes is the fact that, of these people, only around one half are known to have the condition. This has been shown repeatedly in epidemiological surveys and might be due to the absence of symptoms with T2DM. An added concern is that half of those who do present with T2DM clinically already have signs of the complications of the disorder. Earlier detection of diabetes related symptoms might slow down or prevent the development of full blown T2DM and its long-term complications.

Thus, measures that can prevent whenever possible and/or control T2DM and its complications and maximize quality of life are warranted. Treatment of diabetes involves lowering blood glucose and levels of other known risk factors. Additionally, management of T2DM should be supported by lifestyle measures such as a healthy diet and regular physical activity. Changes in lifestyle, however, are not always enough to control blood glucose levels. Therefore, a better understanding of the exact underlying cause of T2DM is needed and could result in the development of more specific therapeutic or even preventive strategies for people who are at risk for T2DM.

\section{Scientific background}

Driven by rapid urbanization, excessive caloric intake, and increasingly sedentary lifestyles, the diabetes epidemic has grown in parallel with the worldwide rise in obesity. Thus, the increase in diabetes prevalence is a reflection of the (still growing) number of people that are overweight. In obesity, the accumulation of excessive fat in non-adipose tissues such as skeletal muscle, liver, and heart, is associated with negative health impacts. As such, fat 
accumulation in skeletal muscle is very strongly associated with the development of insulin resistance, ultimately leading to the development of T2DM. Excess lipid accumulation in skeletal muscle, not matched by improved oxidative capacity, can result in toxic effects on its function and is therefore also referred to as lipotoxicity. Since skeletal muscle is a key insulin-responsive organ responsible for maintaining normal glucose homeostasis, its transition towards an insulin resistant state is an important contributor to the disturbances in glucose metabolism found in T2DM patients. In this context, human studies point towards a role for impaired skeletal muscle mitochondrial function in (pre-)diabetic subjects. This has led to the hypothesis that either acquired or inherited reduced mitochondrial function in the (pre-)diabetic state leads to accumulation of fat in muscle, resulting into the development of insulin resistance. The exact causes of insulin resistance are unknown, and nowadays it is still unclear whether mitochondrial dysfunction represents a primary pathological defect or a secondary, physiological adaptation to conditions of insulin resistance. Therefore, the (causal) link between mitochondrial dysfunction and insulin resistance is currently under debate. The mission of the work described in this thesis was to unravel the relation between mitochondrial function, muscular fat content and insulin resistance. By using an in vivo systemic approach to manipulate aspects of mitochondrial function and muscle substrate metabolism, its effects on glucose tolerance and muscle lipid content were studied. The results provided new insights on the putative role of lipotoxicity in the development of mitochondrial dysfunction in muscle under both, healthy and diabetic conditions. These insights could support the exploration for novel targets for pharmaceutical interventions against T2DM.

\section{Target market}

Regarding diabetes research, a better understanding of the relation between muscular fat accumulation, mitochondrial dysfunction, and insulin resistance is needed to direct the development of novel therapies and treatments against diabetes. Additionally, early detection of diabetes could also reduce the severity and frequency of the immediate effects of diabetes, and prevent or delay its long-term complications. These measures might lead to saving of health care resources as a result of reduced levels of care required for diabetes complications; e.g. reduced hospital admissions and lengths of stay.

It would be essential for diabetes management to provide information regarding diabetes research to health institutions as well as people who are at risk for developing T2DM. Successful treatment of diabetes could improve quality of life and long-term health and reduce health care costs. Knowledge transfer could bridge the gaps between the university's research and industry and society. Collaboration between universities and pharmaceutical 


\section{VALORIZATION}

industry could result into a better understanding of the etiology of diabetes and, therefore, also the development of novel therapies and treatments against T2DM. Subsequently, these new treatment strategies could be implemented into the clinic.

Additionally, given the essential role of mitochondria for cellular life, it is of utmost importance to understand how mitochondrial dysfunction is caused. This notion is not only prominent with regard to diabetes, but applies also for physiology and biochemistry in general. Every cell in living organisms depends on adenosine triphosphate (ATP) as a primary energy source. Mitochondria are the most important organelles for energy production by generating ATP through the electron transport chain (ETC) located in the inner mitochondrial membrane (IMM).

\section{$\underline{\text { Activities/products }}$}

The work described in this thesis is executed at the department of Human Biology of the Maastricht University. The mission of this thesis was to reveal important information on the putative mechanisms that protect mitochondria against lipotoxicity and how this relates to muscle glucose homeostasis and insulin resistance. For the purpose of a better understanding of disease processes, animal studies have been conducted in this thesis. Collaborations with the department of electron microscopy and the department of neuropsychology gave infrastructure for the studies on electron microscopy and in vivo DNA electroporation respectively. Furthermore, by close collaboration between team members within the group of Prof. dr. P. Schrauwen animal studies and human data could be combined. This could result into identification of molecular mechanisms via which muscular lipotoxicity may lead to mitochondrial dysfunction and to the validation and translation of this concept in humans. Thus, data obtained in this thesis, along with future experiments, could be combined with human studies in order to obtain more insight in the relevance of the mechanisms via which muscular lipotoxicity may lead to mitochondrial dysfunction for the etiology of T2DM in humans. To this end, intervention studies need to be done to investigate whether lowering muscular fat content in (pre-)diabetic subjects that are already characterized by mitochondrial dysfunction will result in a restoration of mitochondrial function.

The results obtained in this thesis have been implemented in original scientific research articles. These articles have been published or submitted to professional scientific journals in order to distribute the results to prominent figures in this emerging field of study. This manner, the described work in this thesis could function as a starting point for future studies concerning mitochondrial dysfunction and/or T2DM. 


\section{$\underline{\text { Innovation }}$}

To date, it is has not been revealed whether mitochondrial dysfunction is either a cause or pathological consequence of insulin resistance conditions. The relation between mitochondrial function, lipotoxicity, and insulin resistance remains to be elucidated. Human data on putative underlying mechanisms are still lacking. By using animal models, several supposed underlying mechanisms have been addressed in this thesis. Various approaches have been conducted in order to manipulate aspects of mitochondrial function and muscle substrate metabolism, and their effects on glucose tolerance and muscle lipid content. State of the art technologies such as respirometry and in vivo DNA electroporation have been used to measure mitochondrial function and effects of local overexpression of genes of interest, respectively. The combination of intervention studies (antioxidants, chemical uncouplers, diet) and genetic manipulation experiments in animal models (UCP3 ablation, in vivo DNA electroporation) provides valuable information on the role of muscular lipotoxicity on mitochondrial function and muscle substrate metabolism with respect to glucose homeostasis and insulin resistance. The use of animal models allowed us to investigate disease states in ways which would be inaccessible in a human patient. These data could be used for future studies on the relevance of mitochondrial lipotoxicity for the etiology of T2DM in humans.

\section{Plan of work}

In this thesis in vivo animal models have been used to study the relation between mitochondrial function, lipotoxicity, and insulin resistance in skeletal muscle. Several aspects of mitochondrial function have been manipulated. However, none of the described treatments did result into improvements in insulin sensitivity. Therefore, the results obtained in this thesis cannot be directly translated into data of clinical relevance. However, the research team of Prof. dr. P. Schrauwen continues the research concerning muscular insulin resistance, lipotoxicity, and mitochondrial dysfunction with the emphasis on T2DM. To validate the concept of mitochondrial lipotoxicity, human intervention studies will be performed to investigate the relation between mitochondrial function and muscular fat content. These studies will be performed in collaboration with the department of Radiology of Maastricht University, where non-invasive magnetic resonance spectroscopy will be performed to investigate the in vivo mitochondrial function and lipid accumulation in muscle, liver, and heart. 
Dankwoord 


\section{DANKWOORD}

\section{Dankwoord}

Ik kan het bijna niet geloven, maar het is zover, mijn boekje is af! Nu rest alleen nog het allerlaatste (last but not least!) onderdeel: mijn dankwoord. Ik heb mijn promotietraject als een waardevolle periode ervaren, zowel op wetenschappelijk als sociaal gebied. Deze periode was niet mogelijk geweest zonder de hulp, steun, en motivatie die ik van velen heb mogen ontvangen. Daarom wil ik bij dezen iedereen bedanken die op welke manier dan ook heeft bijgedragen aan de totstandkoming van dit proefschrift.

Allereerst wil ik mijn (co-)promotoren bedanken voor de begeleiding van mijn promotietraject. Beste Patrick en Joris, ik noem jullie in een zin, omdat naast het feit dat jullie kantoren maar door 1 deur van elkaar worden gescheiden, jullie samenwerking van grote waarde voor mij is geweest. Als eerste begeleiders kon ik altijd bij jullie terecht, en jullie efficiënte communicatie heb ik als zeer prettig ervaren. Matthijs, bedankt voor je wetenschappelijke kennis en enthousiasme. Beste heren, behalve op wetenschappelijk gebied, heb ik ook op de wielrenfiets het nodige van jullie kunnen leren. Zo dus ook dat in moeilijke tijden je af en toe door moet bijten om te komen tot waar je wilt komen. 'Life is like riding a bicyle. To keep your balance, you must keep moving' (Albert Einstein).

Ik bedank ook de leden van de beoordelingscommissie: Prof. dr. Wouter van Marken Lichtenbelt, Prof. dr. Aalt Bast, Dr. Riekelt Houtkooper, Prof. dr. Jaap Keijer, en Dr. Joost Luiken, voor het beoordelen van mijn proefschrift.

En dan natuurlijk mijn paranimfen. Lieve Anja en Astrid, ik ben er heel erg trots op dat jullie tijdens mijn verdediging achter mij staan. Anja, onaangekondigd kreeg je mij ineens als kantoorgenoot er bij. Ondanks dat we maar een korte periode roomies zijn geweest, zijn we elkaar wel blijven zien en ik ben blij dat we nog steeds vriendinnen zijn. Ik heb veel bewondering voor je doorzettingsvermogen en kracht. Astrid, jij hebt mij zowel mentale als fysieke bergen op weten te schreeuwen. We hebben veel mooie fietstochtjes gemaakt samen! Ik kon altijd op je rekenen, ook in moeilijke perioden, en ben je heel erg dankbaar voor je vriendschap, advies en wijze raad.

Beste (oud-)colllega's van het SHOCk team, ik wil jullie heel erg bedanken voor de samenwerking en gezelligheid. De waarde van een team is groter dan de som van individuele vaardigheden. Ik ben er trots op om deel uitgemaakt te hebben van dit team. 
Het secretariaat: Desiree, Claudia en Yolanda, jullie zijn vaak de redders in nood geweest. Wanneer niet duidelijke was wat, waar, of wanneer iets geregeld moest worden, waren jullie altijd bereid om mij te helpen en wisten daar ook altijd een vrolijke draai aan te geven.

Kantoorgenootjes, om te beginnen bij kamer 2.306: Hasibe, Madeleen, Guy V, heel erg bedankt voor de gezellige tijd. Hasibe, jij was een beetje het moedertje van kantoor, bedankt voor al je zorgzaamheid en interesse! Madeleen en Guy, ik heb veel respect jullie gedrevenheid en ambities. Madeleen, veel succes en plezier in Stockholm. Guy, ik heb altijd opgekeken naar hoe jij je carrière als arts en wetenschapper weet te combineren. Veel geluk in Rotterdam! Vanuit kamer 2.306 verhuisde ik naar kamer 0.326. Gill, we zijn maar kort kantoorgenootjes geweest. Onder het motto 'alles komt goed' ben je altijd heel druk en gepassioneerd bezig met je projecten. Ik heb veel respect voor wat je allemaal al bereikt hebt en nog gaat bereiken. Silvie, jij wist ons kantoor nog wel eens om te bouwen tot metamorfose hok. Niemand anders had mij ooit in een roze jurk kunnen krijgen! Dit staat ook meteen symbool voor je kracht en overtuiging. Naast dat je een goede collega was, heb ik genoten van je vriendschap en ben ik je dankbaar voor al je gezelligheid en steun. Dirk, jij kwam er als laatste bij als kantoorgenoot, tijdens mijn meest drukke en af en toe stressvolle tijd. Bedankt voor je verdraagzaamheid. Veel succes en plezier in de SHOCk groep!

Ook gaat mijn dank uit naar alle collega's van HB en BW voor de mooie tijd! De vrijdagmiddagborrels, koffiepraat, legendarische kerstdiners en nieuwjaarsfeestjes, carnaval, weekendjes naar de Ardennen, etentjes, en nog veel meer, hebben er voor gezorgd dat ik een onvergetelijke tijd heb beleeft. Jos, jij bent de centrale factor van HB en BW, bedankt voor het organiseren van de HB borrels en social events! Verder wil ik Sanne, Dorien P, Sabine, Giulio (chocolate is the solution for everything $(-)$, and 42 is the answer), Dorien R, Jasper, Cyril, Anouk, Maarten, Boris, Mark, Lisje, Christel, Anke, Karianna, Roel, Ellen, Florence, Jan-Willem, en iedereen die ik nu ben vergeten om te noemen, bedanken voor de support en gezelligheid.

Mijn cluppie, TTV Maastricht, wil ik heel erg bedanken voor de geweldige tijd! Naast het tafeltennissen, heb ik de $3^{\text {de }}$ helft altijd heel erg gewaardeerd. Ook hebben jullie me heel wat bijgebracht over de Maastrichtse cultuur. TTV Mestreech: Bedaank veur de gezèllegheid!

Mijn nieuwe collega's bij Pharming ben ik heel dankbaar voor hun warme welkom en prettige werksfeer. Jullie interesse en begrip tijdens de laatste loodjes van mijn promotietraject hebben er voor gezorgd dat ik dit proefschrift voorspoedig af heb kunnen ronden. 


\section{DANKWOORD}

Vrienden en familie, hoewel mijn werk niet altijd even makkelijk te volgen was voor iedereen, was er wel altijd interesse en betrokkenheid. Stan en Sandra, Marc en Ellen, we zien elkaar niet vaak, maar ik voel me wel altijd heel erg gesteund door jullie waarvoor ik jullie heel erg dankbaar ben. Opa en oma, en oma, ik ben er heel erg trots op dat ik jullie mijn proefschrift kan laten zien.

Tot slot, de laatste en belangrijkste plaats. Lieve papa en mama, heel erg bedankt voor jullie onvoorwaardelijke steun en begrip. Bedankt dat jullie er altijd voor me zijn. Dankzij jullie ben ik gekomen tot waar ik nu sta. 


\section{List of publications}




\section{Published manuscripts}

el Azzouzi H, Leptidis S, Dirkx E, Hoeks J, van Bree B, Brand K, McClellan EA, Poels E, Sluimer JC, van den Hoogenhof MMG, Armand A, Yin X, Langley S, Bourajjaj M, Olieslagers S, Krishnan J, Vooijs M, Kurihara H, Stubbs A, Pinto YM, Krek W, Mayr M, da Costa Martins PA, Schrauwen P, De Windt LJ. The hypoxia-inducible microRNA cluster miR-199a 214 targets myocardial PPARס and impairs mitochondrial fatty acid oxidation. Cell Metab;18(3):341-54, 2013

van Bree $B^{\star}$, Paglialunga $S^{*}$, Bosma M, Valdecantos MP, Amengual-Cladera E, Jörgensen JA, van Beurden D, den Hartog GJ, Ouwens DM, Briedé JJ, Schrauwen P, Hoeks J. Targeting of mitochondrial reactive oxygen species production does not avert lipid-induced insulin resistance in muscle tissue from mice. Diabetologia;55(10):2759-68, 2012

Timmers S, Nabben M, Bosma M, van Bree B, Lenaers E, van Beurden D, Schaart G, Westerterp-Plantenga MS, Langhans W, Hesselink MK, Schrauwen-Hinderling VB, Schrauwen P. Augmenting muscle diacylglycerol and triacylglycerol content by blocking fatty acid oxidation does not impede insulin sensitivity. Proc Natl Acad Sci U S A.;109(29):117116, 2012.

van Triel JJ, van Bree BW, Roberts DW, Muijser H, Duistermaat E, Woutersen RA, Kuper CF. The respiratory allergen glutaraldehyde in the local lymph node assay: sensitization by skin exposure, but not by inhalation. Toxicology;273(1-3):115-22, 2011. 


\section{Submitted manuscripts}

van Bree B, Paglialunga S, Kornips E, Jörgensen JA, Schrauwen P, Hoeks J. A two-week treatment with 2,4-dinitrphenol does not affect muscle substrate oxidation and glucose homeostasis in WT and db/db mice

Bianca W.J. van Bree, Ellen Lenaers, Miranda Nabben, Jacco J. Briedé, Johanna A. Jörgensen, Gert Schaart, Patrick Schrauwen, Joris Hoeks, Matthijs K.C. Hesselink. A genistein-enriched diet does not improve skeletal muscle oxidative capacity or prevent the transition toward advanced stages of insulin resistance in ZDF rats

B.W.J. van Bree, A.R. Martins, J.A. Jörgensen, E. Kornips, G. Schaart, J. Hoeks, P. Schrauwen. The mitochondrial protein mitoNEET: a study of its role in mitochondrial oxidative capacity, substrate metabolism, and lipid accumulation in skeletal muscle in rats

Bianca W.J. van Bree*, Miranda Nabben*, Ellen Lenaers, Joris Hoeks, Matthijs K. C. Hesselink, Gert Schaart, Marion J.J. Gijbels, Jan F.C. Glatz, Gustavo J.J. da Silva, Leon J. de Windt, Rong Tian, Elise Mike, Darlene G. Skapura, Xander H.T. Wehrens, and Patrick Schrauwen. Lack of UCP3 does not affect skeletal muscle mitochondrial function under lipidchallenged conditions, but leads to sudden cardiac death 
Curriculum Vitae 


\section{Curriculum Vitae}

Bianca van Bree was born on August $14^{\text {th }} 1984$ in Boxmeer, The Netherlands. In 2002, she finished pre-university secondary education at Elzendaal College in Boxmeer. Subsequently, she started with the bachelor Biochemistry at Hogeschool van Arnhem en Nijmegen and graduated in 2006.

In 2006 Bianca continued her study with the pre-master Biomedical Sciences at the Radboud Universiteit in Nijmegen. Thereafter, she started with the master Biomedical Sciences at the same university and specialized in both 'Human Pathobiology' and 'Toxicology'. As part of her MSc training, she performed an internship at the Department of Cellular Biochemistry at the Netherlands Cancer Institute in Amsterdam, where she participated in studies to characterize autotaxin and LPA receptor signalling in cancer. Her second internship was at the Department Toxicology and Applied Pharmacology at TNO Quality of Life in Zeist, during which she investigated the potential and mechanisms of compounds to induce respiratory allergy. She graduated in September 2009.

In November 2009, she started her PhD at the Department of Human Biology at Maastricht University. Her project focusing on skeletal muscle mitochondrial function and lipotoxicity in relation to insulin resistance was supervised by Prof. P. Schrauwen, Prof. M. Hesselink, and Dr. J. Hoeks.

Bianca van Bree is currently working as a Scientist at the R\&D department of Pharming Group NV in Leiden. 